Optically Resonant Structures for the Enhancement of Polycrystalline

PbSe Photoconductors

A Dissertation Presented to the Faculty of the Graduate School

University of Missouri-Columbia

In Partial Fulfillment of the Requirements for the Degree

Doctor of Philosophy

by

Justin Stewart Grayer

Dr. Randy Curry, Dissertation Supervisor

December 2018 
The undersigned, appointed by the dean of the Graduate School, have examined the dissertation entitled

\section{Optically Resonant Structures for the Enhancement of Polycrystalline PbSe Photoconductors}

presented by Justin Stewart Grayer, a candidate for the degree of doctor of philosophy, and hereby certify that, in their opinion, it is worthy of acceptance.

\section{Dr. Randy Curry}

Department of Electrical and Computer Engineering

Dr. David Bryan

Department of Electrical and Computer Engineering

Dr. Justin Legarsky

Department of Electrical and Computer Engineering

Dr. Mark Prelas

Department of Electrical and Computer Engineering

Dr. Jacob A. McFarland

Department of Mechanical and Aerospace Engineering 
Dedicated to Sarah. 


\section{Acknowledgements}

My journey into higher education started over 13 years ago, so there are too many people to thank for the successes and experience gained along the way here. Instead, I would like to specifically acknowledge a few major influencers and supporters. First I would like to thank my mother, Carla. You raised me, gave me the means and encouragement to support myself, and have been in my corner through this whole process. As my first teacher, you instilled in me the drive and will to pursue my dreams. Without you, this achievement would not have been possible.

I would also like to thank the teachers, instructors, and professors I have learned from throughout my academic career. I have been influenced by you all, and my academic success serves as a testament to your expertise and ability to pass on your knowledge to your students. I want to especially acknowledge Dr. Gavin King. Thank you for giving a technician the chance to learn and grow in the early years of your laboratory. The opportunities you helped provide me enabled me to gain the knowledge and experience I needed to become a better-rounded engineer.

The Professor I would like to thank most of all is my advisor, Dr. Randy Curry. Though you have only been my advisor for a short time, you have been a mentor to me for several years, and it has been a pleasure getting to know and learn from you. Thank you for expanding my scientific understanding into the realm of high power electromagnetics, and giving me the freedom to explore my own path. Most of all, thank you for agreeing to be my advisor and helping me through this process. I would also like to give my sincerest gratitude to Dr. David Bryan. Thank you for reading and 
commenting on the many drafts of this dissertation. Your suggestions were invaluable in bringing this document to what it is today.

I would like to give a special thanks to my WIRED team members and collaborators for helping with film growth, fabrication, and early characterization studies of our PbSe films. I would also like to thank the DARPA MTO program office and the Air Force Reasearch Laboratory (AFRL) for your valuable suggestions and insight during this investigation.

The most important person to acknowledge and give my most sincere gratitude would be my friend, my rock, my wife, Sarah. You have been there since the beginning, and have stayed on this journey with me over the years. This process has been long, and at times challenging. Yet, you have encouraged me, built me up, and bolstered my resolve when I needed it. Thank you for all that you've done, all that you do, and all that you are.

Distribution Statement A: Approved for Public Release; Distribution is Unlimited; Northrop Grumman Corporation \#18-2205; Dated 10/23/2018.

This research was developed with funding and sponsorship from the Defense Advanced Research Projects Agency (DARPA) and the Air Force Research Laboratory (AFRL).

The views, opinions and/or findings expressed are those of the author and should not be interpreted as representing the official views or policies of the Department of Defense or the U.S. Government. 


\section{Contents}

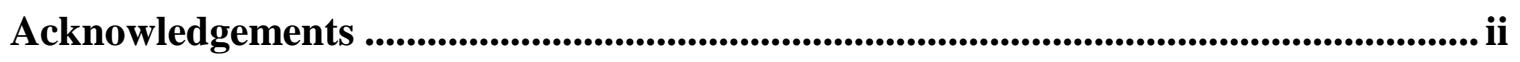

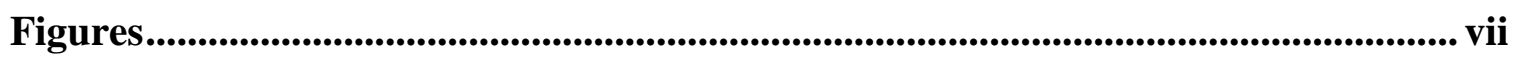

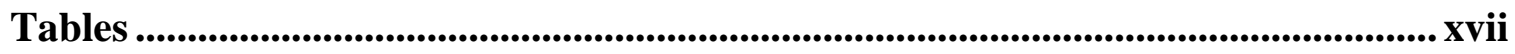

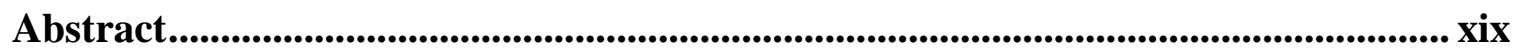

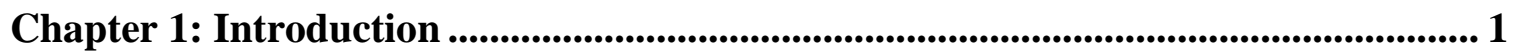

1.1 Introduction of Lead Selenide ................................................................ 1

1.2 Resonant Structures for Optical Management ................................................. 4

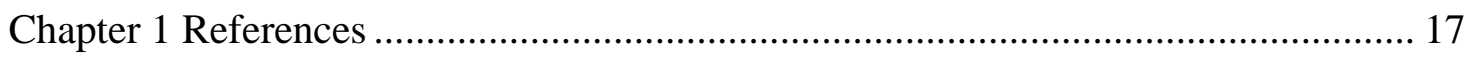

Chapter 2: Lead Selenide (PbSe) as a Detector Material.......................................... 23

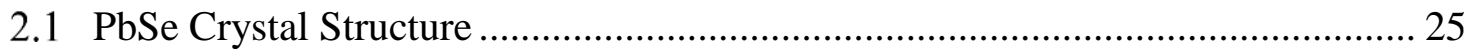

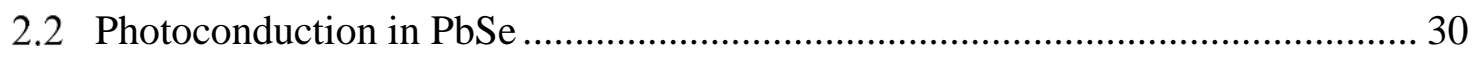

2.3 Absorption in $\mathrm{PbSe}$................................................................................ 45

2.4 Performance of Baseline PbSe Test Detectors............................................. 49

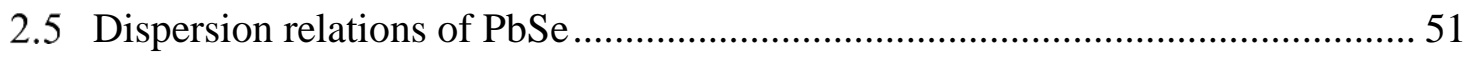

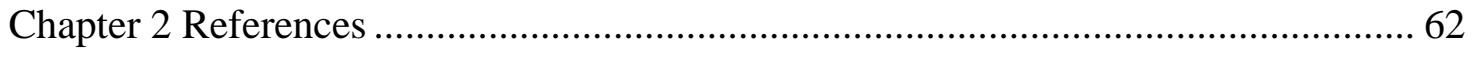

Chapter 3: Surface Plasmon Resonance ....................................................................68

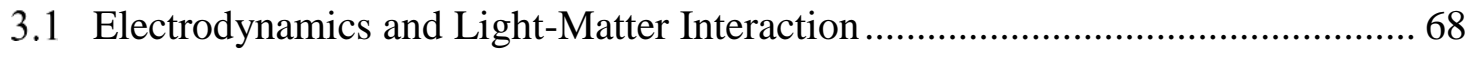

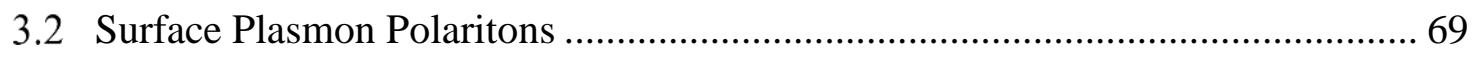

3.3 Investigating the Plasmonic Response in PbSe ......................................... 85

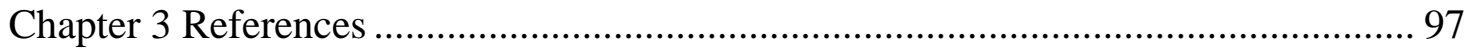


4.1 Derivation of the Refractive Index and Absorption Coefficient....................... 102

4.2 Absorption in PbSe Thin Films............................................................ 105

4.3 Thin Film Interference ............................................................................. 108

4.4 The Back Reflector Structure in PbSe .......................................................... 114

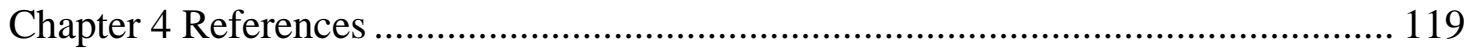

Chapter 5: Design Methodology and Performance Predictions .............................. 122

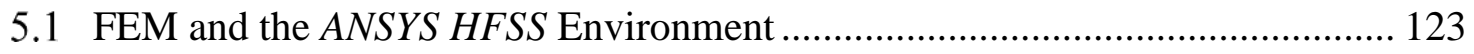

5.2 Modeling the PbSe Film with ANSYS HFSS ........................................... 127

5.3 Predicted Results for Metal Discs in PbSe Films ........................................ 134

5.4 Predicted Results for an Embedded Reflector Layer in PbSe Films ................ 149

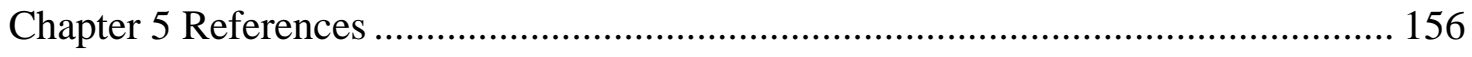

Chapter 6: PbSe Film and Device Measurements ................................................ 159

6.1 Confirmation of Plasmonic Resonance of Metal Discs in PbSe...................... 159

6.2 PbSe Detectors with Embedded Pt Disc Arrays .......................................... 166

6.3 PbSe Devices with Embedded Metallic Back Reflector ................................ 171

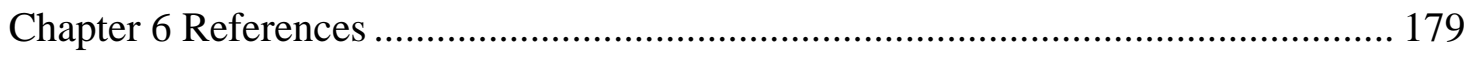

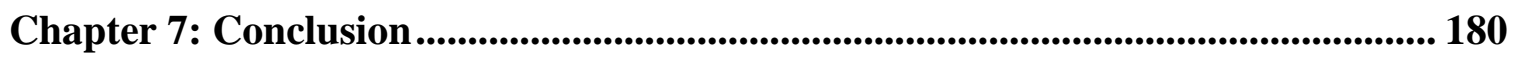

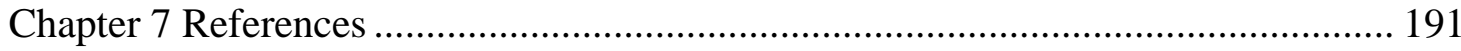

Appendix A: Fabrication of PbSe Devices................................................................ 192

PbSe Baseline Detector Fabrication............................................................. 192

Preparation for Resonant Structures: PbSe Roughness Reduction .......................... 194 
Appendix A References

Appendix B: Surface Plasmon Resonance Numerical Models

Quasi-static Calculation of the Scattering, Absorption, and Extinction Cross-sections

and Q-Factors for Surface Plasmon Resonant Discs ............................................. 201

Calculation of the Dispersion Relations of Drude Metals ..................................... 202

Surface Plasmon Propagation Distance, Phase Velocity, and Lifetime.................... 207

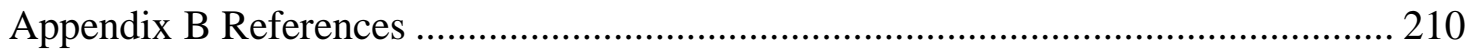

Appendix C: Infrared Sensor System Considerations .......................................... 211

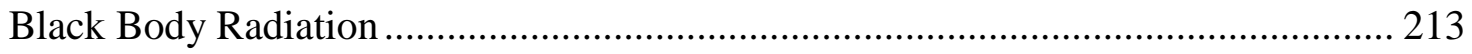

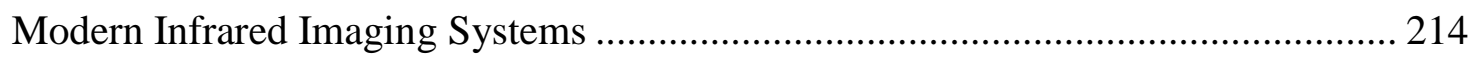

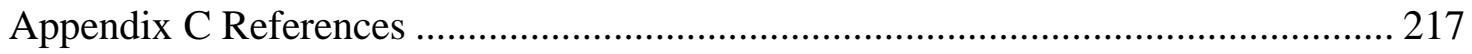

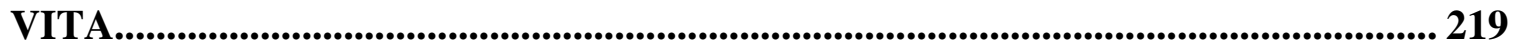




\section{Figures}

Figure 1. Depiction of a) surface plasmon polaritons at the metal/dielectric interface, b) coupled oscillations of free electrons in a metal surface feature in response to a resonant

electric field, and c) surface plasmon polariton dispersion relations $[34,54] \ldots \ldots \ldots \ldots \ldots . . .7$

Figure 2. Interference in idealized $\mathrm{PbSe}$ thin films on $\mathrm{Si}$ substrate.............................. 13

Figure 3. PbSe films grown by chemical bath deposition for this investigation; a) Shown from left to right are the Si substrate (top-left), as-grown PbSe film (bottom-left), oxidized PbSe film (top-right), and sensitized PbSe film (bottom-left); b) Depiction of sensitized PbSe film cross-section.

Figure 4. EDS map depicting the polycrystalline nature of, as well as the interface between, the $\mathrm{PbSe}$ and $\mathrm{PbI}_{2}$ within the film after sensitization [1]............................ 26

Figure 5. Depiction of crystal structure of PbSe films; a) as-grown $\mathrm{PbSe}, \mathrm{b}$ ) monoclinic $\mathrm{Pb}_{3} \mathrm{O}_{2}\left(\mathrm{SeO}_{3}\right)$, c) orthorhombic $\mathrm{Pb}_{3} \mathrm{O}_{2}\left(\mathrm{SeO}_{3}\right)$, d) polytype $12 \mathrm{R}^{\mathrm{PbI}_{2}}$ [2].

Figure 6. Secondary electron images obtained using a scanning electron microscope on the of a) oxidized, and b) sensitized PbSe films of this study. During the iodine sensitization process, crystallite size is reduced while the number and size of crystallite agglomerations increases. 28

Figure 7. HRTEM images collected on sensitized PbSe films of this study. a) PbSe crystallite agglomerations after sensitization, b) carbon shell surrounding PbSe crystallite for $400{ }^{\circ} \mathrm{C}$ oxidation, and c) reduced carbon shell after $420{ }^{\circ} \mathrm{C}$ oxidation. 29

Figure 8. The XRD spectra of oxidized PbSe films as a function of time and temperature $[2,9]$. When the oxidization temperature is increased from a) $400{ }^{\circ} \mathrm{C}$ to b) $460{ }^{\circ} \mathrm{C}$, the 
$\mathrm{PbSeO}_{3}$ compound is converted to $\mathrm{PbSeO}_{4}$. Longer times can lead to over oxidation, and decreased film sensitivity. 29

Figure 9. Qualitative band diagram sketch of PbSe photoconductive films obtained using the electron affinity rule. When $\mathrm{PbSe}$ and $\mathrm{PbI}_{2}$ form an interface, the Fermi-level of the system lowers enabling increased hole flow

Figure 10. Van der Pauw configuration for measuring Hall parameters; b) PbSe device for Hall measurements where the metal contacts are placed in the four-corners of the

$\mathrm{PbSe}$ film

Figure 11. Temperature dependent a) carrier concentration, b) carrier mobility, c) film resistivity, and d) Hall coefficient in sensitized PbSe films. Trends indicate sensitivity

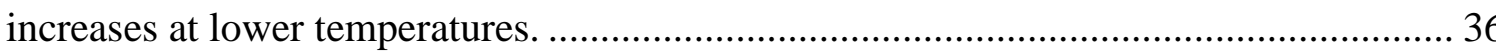

Figure 12. Bias circuit for measuring minority carrier lifetime in PbSe films. 39

Figure 13. The room temperature PCD curve observed by transversely illuminating a $\mathrm{PbSe}$ test detector with pulsed laser light, and measuring the change in voltage across a load resistor connected in series. The carrier lifetime was extracted by fitting an

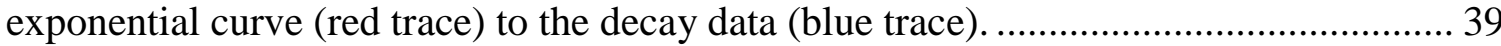

Figure 14. An RC-time constant measurement setup. ............................................. 40

Figure 15. The RC-decay curve measured across the PbSe detector as it was pulsed by an AWG. The decay curve shows a factor of two increase over that of the PCD measurement, distinguishing the measured lifetime from the RC time constant. 41 Figure 16. Time resolved photoluminescence setup for carrier lifetime measurements on $\mathrm{PbSe}$ test detectors. 42 
Figure 17. Observed TRPL decay curve for PbSe test detector at room temperature.

Results show a three-order-of-magnitude disparity compared to those of the PCD method due to carrier trapping. 43

Figure 18. Observed temperature and bias dependence of the carrier lifetime of PbSe test detectors measured using the PCD method. 44

Figure 19. Depiction of FTIR measurement. 45 Figure 20. Absorption spectrum of investigated PbSe thin films at $290 \mathrm{~K}$ determined by FTIR with integrating sphere. 48

Figure 21. Reported Thor Labs responsivity (blue, dashed), and calculated FTIR source power spectrum (red, solid).

Figure 22. Measured responsivity and external quantum efficiency of a $100 \mu \mathrm{m}$ x $40 \mu \mathrm{m}$ $\mathrm{PbSe}$ test detector under an applied electric field of $3.3 \mathrm{kV} / \mathrm{cm}$ at ambient temperatures of interest. 50 Figure 23. Graphical depiction of photoluminescence process in a direct bandgap semiconductor: a) electrons are excited to a state above the bottom of the conduction band upon absorption of pump photons; b) the electron undergoes non-radiation relaxation down to the bottom of the conduction band; c) an exciton is created between the electron at the bottom of the conduction band and holes at the top of the valence band; d) the electron relaxes from the metastable state recombining with the hole and the

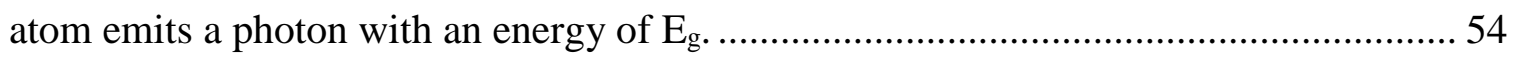
Figure 24. Graphical depiction of a photoluminescence setup................................... 55 Figure 25. Measured temperature dependent photoluminescence of sensitized PbSe films. 
Figure 26. The real part of the index of refraction of PbSe from $2.5 \mu \mathrm{m}$ to $6 \mu \mathrm{m}$ at

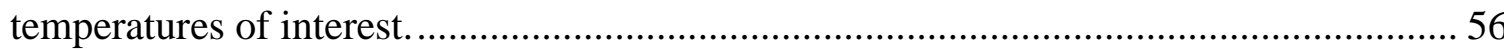

Figure 27. The imaginary part of the index of refraction of PbSe between $2 \mu \mathrm{m}$ and $6 \mu \mathrm{m}$

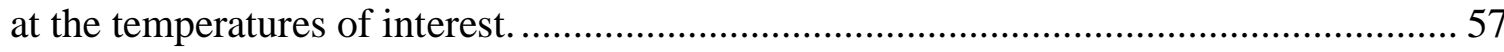

Figure 28. Modified dispersion relations of PbSe. ................................................. 59

Figure 29. Depiction of a prolate spheroid, which is an analogue of the rod structure.... 80 Figure 30. Depiction of an oblate spheroid, which is an analogue of the disc structure. . 81 Figure 31. Surface plasmon propagation distance for various metals embedded in $\mathrm{PbSe}$.

Figure 32. Phase velocity normalized to the speed of light in vacuum of surface plasmons for various metals in PbSe. 90 Figure 33. Surface plasmon lifetime for various metals in PbSe.

Figure 34. Extinction cross-section of a single $\mathrm{Pt}$ nanodisc at the $\mathrm{PbSe} / \mathrm{SiO}_{2}$ interface. Resonance peak red-shifts with wavelength, demonstrating wavelength selectivity for designing surface plasmon resonant disc structures for detector enhancement.

Figure 35. Calculated scattering-cross section for surface plasmon resonant discs at the $\mathrm{PbSe}-\mathrm{SiO}_{2}$ interface as a function of incident illumination angle.

Figure 36. Field intensity enhancement map of a $\mathrm{Pt}$ disc located at the $\mathrm{SiO}_{2}-\mathrm{PbSe}$ interface illuminated with a $3.7 \mu \mathrm{m}$ plane wave at normal incidence 95 Figure 37. Calculated wavelength-dependent absorption coefficient of PbSe films..... 106 Figure 38. Comparison of absorption calculated from modified PbSe dispersion relations and measured absorption in multi-layer PbSe films. 107 
Figure 39. Interaction of a light wave as it transmits through the layers of an optical thin film 108

Figure 40. Depiction of PbSe film cross-section with embedded Pt back reflector. ...... 115 Figure 41. Calculated absorption in modeled PbSe films with $\mathrm{Pt}$ back reflector for various $\mathrm{SiO}_{2}$ thicknesses. 116

Figure 42. Calculated absorption enhancement in $\mathrm{PbSe}$ films with back reflector as a function of $\mathrm{SiO}_{2}$ thickness. 118

Figure 43. Simple depiction of the finite element method (FEM) for solving electromagnetic problems. 123

Figure 44. Simple depiction of adaptive mesh process in ANSYS HFSS. 126

Figure 45. Simple flow block diagram for the FEM and adaptive mesh refinement in ANSYS HFSS.

Figure 46. Unit cell of the PbSe films under investigation modeled in ANSYS HFSS. 127 Figure 47. Periodic Master/Slave boundary conditions for modeled PbSe films.... 129 Figure 48. Modeled absorption in $\mathrm{PbSe}$ thin films with $1225 \mathrm{~nm} \mathrm{SiO} 2,500 \mathrm{~nm} \mathrm{PbSe}$, and 400 nm $\mathrm{PbI}_{2}$ 132

Figure 49. Comparison of modeled to measured absorption in PbSe films for model validation 133 Figure 50. Efficiency (Q) factors of $\mathrm{Pt}$ discs at $\mathrm{SiO}_{2} / \mathrm{PbSe}$ interface for an aspect ratio of 12.5 135 Figure 51. Results of the parametric analysis of the Au and Pt discs embedded at the $\mathrm{SiO}_{2}-\mathrm{PbSe}$ interface. 136 
Figure 52. Comparison of $20 \mathrm{~nm}, 80 \mathrm{~nm}$, and $160 \mathrm{~nm}$ thick Au discs with fixed aspect ratios.

Figure 53. Comparison of $20 \mathrm{~nm}, 80 \mathrm{~nm}$, and $160 \mathrm{~nm}$ thick Pt discs with fixed aspect ratios.

Figure 54. $\mathrm{PbSe}_{-} \mathrm{SiO}_{2}$ interface unit cell with single Pt disc modeled in ANSYS HFSS.

Figure 55. Extinction efficiency (Q) factor, dominated by scattering, of Pt discs at the $\mathrm{SiO}_{2}-\mathrm{PbSe}$ interface modeled in ANSYS HFSS.

Figure 56. Extinction efficiency (Q) factor, dominated by scattering, of Au discs at the $\mathrm{SiO}_{2}-\mathrm{PbSe}$ interface modeled in ANSYS HFSS.

Figure 57. Comparison of $\mathrm{Au}$ and $\mathrm{Pt}$ dispersion relations from $2.5 \mu \mathrm{m}$ to $6 \mu \mathrm{m}$.

Figure 58. Modeled electric field enhancement plots in the (top-left) xz-, (top-right) yz-, and (bottom) xy-directions for a single $\mathrm{Pt}$ disc at $\mathrm{SiO}_{2} / \mathrm{PbSe}$ interface. 144 Figure 59. Modeled electric field intensity enhancement plots in the (top-left) xz-, (topright) $\mathrm{yz}-$, and (bottom) xy-directions for a single $\mathrm{Au}$ disc at $\mathrm{SiO}_{2} / \mathrm{PbSe}$ interface. 145 Figure 60. The modeled unit cell of the PbSe film with embedded metallic discs modeled in ANSYS HFSS. 146

Figure 61. Modeled absorption in $\mathrm{PbSe}$ films with metallic (PEC) discs at $\mathrm{SiO}_{2} / \mathrm{PbSe}$ interface

Figure 62. Effect of $\mathrm{SiO}_{2}$ and $\mathrm{PbI}_{2}$ layer thickness on absorption in $\mathrm{PbSe}$ Films with $\mathrm{Pt}$ back reflector in configuration 1

Figure 63. Effect of $\mathrm{SiO}_{2}$ and $\mathrm{PbI}_{2}$ layer thickness on absorption in $\mathrm{PbSe}$ Films with $\mathrm{Pt}$ back reflector in configuration 2 
Figure 64. Effect of $\mathrm{SiO}_{2}$ and $\mathrm{PbI}_{2}$ layer thickness on absorption in $\mathrm{PbSe}$ Films with $\mathrm{Pt}$ back reflector in configuration 3 152

Figure 65. Electric field and field intensity enhancement due to interference at $4.08 \mu \mathrm{m}$ in PbSe films with Pt back reflector, $1500 \mathrm{~nm} \mathrm{SiO}$, and $300 \mathrm{~nm} \mathrm{PbI}_{2}$ thickness 153 Figure 66. Predicted sensitivity of $\mathrm{PbSe}$ detector with $\mathrm{Pt}$ back reflector compared to baseline result. 154 Figure 67. SEM images of $500 \mathrm{~nm}$ (left) and $1000 \mathrm{~nm}$ (right) disc arrays patterned by EBL. 160 Figure 68. Optical density spectra of $\mathrm{Pt}$ (top) and $\mathrm{Au}$ (bottom) disc arrays on $\mathrm{SiO}_{2}$ layer and Si substrate. 161

Figure 69. Comparison of modeled and measured results for $500 \mathrm{~nm} \mathrm{Au}$ disc array on $\mathrm{SiO}_{2}$ layer and $\mathrm{Si}$ substrate at room temperature.

Figure 70. Comparison of modeled and measured results for $1000 \mathrm{~nm}$ Au disc array on $\mathrm{SiO}_{2}$ layer and $\mathrm{Si}$ substrate at room temperature. 163

Figure 71. Room temperature comparison of resonance for $500 \mathrm{~nm}$ diameter $\mathrm{Au}$ disc arrays within and without $\mathrm{PbSe}$ films with layer thicknesses of approximately $400 \mathrm{~nm}$, $500 \mathrm{~nm}$, and $1200 \mathrm{~nm}$ for the $\mathrm{PbI}_{2}, \mathrm{PbSe}$, and $\mathrm{SiO}_{2}$, respectively.

Figure 72. Comparison of modeled and measured spectra of $500 \mathrm{~nm} \mathrm{Au} \mathrm{disc} \mathrm{arrays} \mathrm{in}$ PbSe films.

Figure 73. PbSe photoconductive devices with embedded Pt disc array; (top-left) depiction of the designed device cross-section, (top-right) depiction of the top view of device showing the Pt disc arrays, and (bottom) top view of fabricated PbSe device. .. 166 
Figure 74. Comparison of room temperature (290 K) spectral response (top) and EQE (bottom) between PbSe with embedded Pt discs and baseline detectors. 168

Figure 75. Comparison of room temperature (290 K) spectra of PbSe with embedded $\mathrm{Pt}$ discs with that of the $230 \mathrm{~K}$ spectra of baseline detectors. 169 Figure 76. PbSe photoconductive devices with embedded Pt back reflector; (left) depiction of the designed device cross-section, (right) top view of fabricated $\mathrm{PbSe}$ device.

Figure 77. Room temperature (290 K) spectral response of PbSe test detectors with embedded Pt back reflector and $1.5 \mu \mathrm{m} \mathrm{SiO} 2$ spacer under an applied field of $1 \mathrm{kV} / \mathrm{cm}$.

Figure 78. Comparison of room temperature performance of baseline $\mathrm{PbSe}$ and predicted performance with $\mathrm{Pt}$ back reflector at $3 \mathrm{kV} / \mathrm{cm}$. 174 Figure 79. Comparison of $290 \mathrm{~K}$ spectra of PbSe with embedded Pt reflector with that of the $250 \mathrm{~K}$ spectra of baseline detectors. 176 Figure 80. Measured vs modeled spectral response at $290 \mathrm{~K}$ of PbSe test detectors with embedded Pt back reflector and $1.5 \mu \mathrm{m} \mathrm{SiO}_{2}$ layer. 177 Figure 81. Unit cell of PbSe film with combination of surface plasmon resonant Pt discs and Pt back reflector modeled in ANSYS HFSS. 181 Figure 82. Predicted absorption in PbSe films with combined optical enhancement techniques of SPR and embedded metallic back reflector compared to the modeled absorption in baseline films. 182 
Figure 83. Predicted absorption enhancement in PbSe films with combined optical enhancement techniques of SPR and embedded metallic back reflector compared to the modeled absorption in baseline films. 183

Figure 84. Electric field Intensity enhancement plots for surface plasmon resonant Pt discs and Pt back reflector embedded in PbSe films at an incident wavelength of $3.60 \mu \mathrm{m}$. in the (top-left) xz-, (top-right) yz-, and (bottom) xy-directions. 184 Figure 85. Electric field intensity enhancement plots for surface plasmon resonant Pt discs and Pt back reflector embedded in PbSe films at an incident wavelength of $4.48 \mu \mathrm{m}$. in the (top-left) xz-, (top-right) yz-, and (bottom) xy-directions. 185

Figure 86. Predicted room temperature spectral responsivity (solid) and quantum efficiency (dashed) of PbSe devices with SPR disc arrays and embedded back reflector compared to the current baseline detector performance.

Figure 87. Predicted room temperature spectral responsivity enhancement $\mathrm{PbSe}$ devices with SPR disc arrays and embedded back reflector (blue, solid) over the baseline detector performance (black, dashed). 188

Figure 88. Predicted room temperature (290 K) sensitivity of PbSe detectors with combined Pt disc array and Pt back reflector compared to measured baseline sensitivity at $230 \mathrm{~K}$ 189

Figure 89. Fabrication process for PbSe photoconductive devices. 193

Figure 90. SEM image of $\mathrm{SiO}_{2}$ surface after polystyrene nanosphere spin coating. ...... 196 Figure 91. Measured surface roughness maps obtained using AFM of smooth (top-left), baseline roughened (top-right), and nanosphere lithography roughened (bottom) $\mathrm{SiO}_{2}$ on Si substrates. 197 
Figure 92. Measured surface profiles obtained using AFM of smooth (top), baseline roughened (middle), and nanosphere lithography roughened (bottom) $\mathrm{SiO}_{2}$ on $\mathrm{Si}$

substrates.

Figure 93. Transmission spectra from visible to LWIR regions of the electromagnetic spectrum over a $1 \mathrm{~km}$ range at an altitude of $5 \mathrm{~km}$ [4]. Each spectral region contains atmospheric windows through which electromagnetic radiation can propagate without substantial absorption from atmospheric particulates.

Figure 94. The $300 \mathrm{~K}$ black body emission spectrum calculated using Plank's law. Thought the peak of the spectrum is located at $9.8 \mu \mathrm{m}$, there is appreciable emission in the MWIR regime which typically drives the need for a cooling element in infrared sensing systems.

Figure 95. Block diagram showing emitted photons from an object of interest as they transmit through the atmosphere into a long-range sensing system. Photons are attenuated by atmospheric particulates, as well as the optical elements of the sensing system...... 215 


\section{Tables}

Table 1. Calculated interface reflectance and reflection-induced phase shifts in

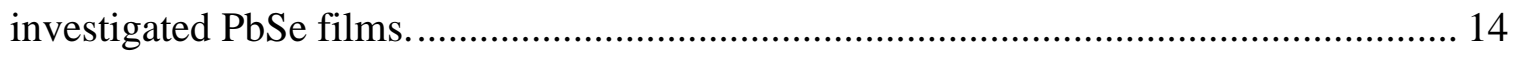

Table 2. PbSe film characteristics determined by Hall measurements........................... 35

Table 3. Bauer and Krenn's PbSe dispersion relations at an ambient temperature of 290

$\mathrm{K}$

Table 4. Temperature dependent scaling factor calculated from the PL spectra of

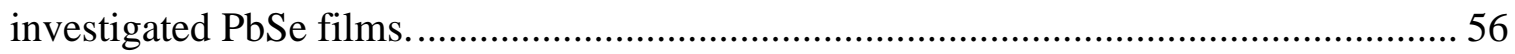

Table 5. Calculated surface plasmon characteristics for various metals embedded in $\mathrm{PbSe}$.

Table 6. Calculated absorption coefficient using the dispersion relations from figure 28

for PbSe films. 105

Table 7. Layer thicknesses for modeled PbSe films with Pt back reflector. 115

Table 8. Specified analysis setup parameters for modeled PbSe film simulations in ANSYS HFSS.

Table 9. Selected parameters for finite element method modeling of single $\mathrm{Au}$ and $\mathrm{Pt}$ discs....... 138

Table 10. Thickness parameters for three different configurations of $\mathrm{PbSe}$ films used to observe the effect of the $\mathrm{PbI}_{2}$ and $\mathrm{SiO}_{2}$ layer thickness on the absorption in $\mathrm{PbSe}$ films with a Pt back reflector.

Table 11. Measured disc diameter and pitch for $500 \mathrm{~nm}$ and $1000 \mathrm{~nm} \mathrm{Au}$ and Pt discs patterned by EBL. 160 
Table 12. Unit cell parameters for modeling the combination of surface plasmon resonant Pt discs and Pt back reflector in PbSe films. ........................................................ 181 


\begin{abstract}
The mid-wave infrared (MWIR) regime of the electromagnetic spectrum is attractive for long-range imaging systems due to the atmospheric window between 3 and $5 \mu \mathrm{m}$. Due to ambient thermal background, it is often necessary to operate sensor systems below room temperature to achieve an adequate signal-to-noise ratio (SNR). This cooling requirement adds size, weight, and complexity to systems in which these parameters are at a premium. In this work I investigated two methods for optically enhancing the absorptive properties of lead selenide $(\mathrm{PbSe})$ photoconductive films to increase the operating temperature up to $290 \mathrm{~K}$, thereby mitigating system cooling requirements. By employing surface plasmon resonant (SPR) and embedded reflective structures, we were able to demonstrate enhanced responsivity and raise the operating temperature to roomtemperature. Sensitivity was observed to increase by a factor of three for SPR enhanced detectors, and up to two-times at room temperature in detectors with an embedded $\mathrm{Pt}$ back reflector. Moreover, PbSe detectors with SPR discs operating at room temperature were observed to have responsivity comparable to reference detectors at $230 \mathrm{~K}$. Photoconductors with the embedded Pt back reflector had a performance at room temperature that was similar to the reference detector at $250 \mathrm{~K}$. Herein, I discuss my design process, as well as the fabrication of these resonant structures. Also discussed are the measurement and test results I obtained from surface plasmon and embedded reflector enhanced $\mathrm{PbSe}$ detectors. In this dissertation, I present results that demonstrate the viability of SPR and interference structures as mechanisms for increasing the operating temperature of PbSe MWIR photodetectors up to $290 \mathrm{~K}$.
\end{abstract}




\section{Chapter 1: Introduction}

\subsection{Introduction of Lead Selenide}

Materials like mercury cadmium telluride (HgCdTe), indium antimonide (InSb), and lead selenide ( $\mathrm{PbSe}$ ) are often employed as mid-infrared (MWIR) materials [1-4, 16-19]. Due to the large amount of ambient thermal background between $3 \mu \mathrm{m}$ and $5 \mu \mathrm{m}$ of the electromagnetic spectrum, MWIR detector materials are typically operated at cryogenic temperatures to minimize noise. This cooling requirement adds cost and complexity to a detector subsystem. For instance, $\mathrm{HgCdTe}$ and $\mathrm{InSb}$ operate at $77 \mathrm{~K}$, which requires an integrated cryocooler adding size, weight, and power draw to the system. Further, the cost of the cryocooler system can often times be a significant fraction of the total cost of the system, including a decrease in reliability and lower lifetime. In these detector materials performance is prioritized over system cost and complexity, limiting their use and utility to research and defense applications. However, there is now a new focus in military, commercial, and consumer industries for near-room temperature, high sensitivity IR detector technology. As an alternative to $\mathrm{HgCdTe}$ and InSb, PbSe-based detectors can serve to mitigate the stringent cooling requirement.

Polycrystalline PbSe is a IV-VI material typically employed for MWIR detectors. $\mathrm{PbSe}$ is grown using well-established chemical bath deposition techniques, and photodetectors based on $\mathrm{PbSe}$ operate at temperatures between $230 \mathrm{~K}$ and $250 \mathrm{~K}$ for adequate signal-to-noise performance [5-9, 11, 13, 14-16]. Sensitivity is an important figure of merit when discussing infrared detectors. The specific detectivity $\mathrm{D}^{*}$, a measure of detector sensitivity, of PbSe based detectors has been reported to be as high as $4 \times$ 
$10^{10} \mathrm{~cm} \sqrt{\mathrm{Hz}} / \mathrm{W}$ at $230 \mathrm{~K}[14,15]$, making its near room temperature performance comparable to other detector materials, such as InSb [16], at cryogenic temperatures. Specific detectivity, with units of $\mathrm{cm} \cdot \sqrt{\mathrm{Hz}} / W$ or Jones, for a detector material is given by equation 1.1 ,

$$
D^{*}=\frac{\sqrt{A_{d} \Delta f} \eta_{e} q}{V_{n} h v}=\frac{\sqrt{A_{d} \Delta f} \Re(h v)}{V_{n}}
$$

where $\mathrm{A}_{\mathrm{d}}$ is the area of the detector in $\mathrm{cm}^{2}, \Delta f$ is the bandwidth in $\mathrm{Hz}, \eta_{e}$ is the external quantum efficiency, q is the electric charge constant $\left(1.602 \times 10^{19} \mathrm{C}\right), \mathrm{V}_{\mathrm{n}}$ is the measured noise typically given in units of current or voltage depending on the type of measurement, and $h v$ is the energy of the photons in joules incident on the detector [3, 16]. The variable $\Re(h v)$ in equation 1.1 is the energy dependent responsivity, which can also be used as a standalone figure of merit for detector sensitivity. This quantity has been utilized as one of the major figures of merit during this investigation.

In an alternative treatment proposed by Phillips, $\mathrm{D}^{*}$ can be related to the carrier density $\mathrm{n}_{\text {th }}$ in $\mathrm{cm}^{-3}$, the absorption coefficient $\alpha$ in $\mathrm{cm}^{-1}$, and carrier lifetime $\tau$ given in seconds of the detector material as shown in equation 1.2 [9].

$$
\mathrm{D}^{*}=\frac{\eta_{\mathrm{e}} \mathrm{q}}{\mathrm{hv} \sqrt{\frac{2 \mathrm{n}_{\mathrm{th}}}{\alpha \tau}}}
$$

An examination of Phillips model in equation 1.2 shows that the sensitivity of a detector material can be improved by increasing the material absorption, yet this parameter is a material dependent property which cannot be readily improved without changing the material system. As an alternative, structures based on surface plasmon resonance and thin film interference have been embedded into PbSe films to affect their 
absorptive properties with the goal of increasing the operating temperature from between $230 \mathrm{~K}$ and $250 \mathrm{~K}$ up to $290 \mathrm{~K}$ without affecting the material composition.

Herein, I demonstrate up to three-times the sensitivity of reference detectors as a result of embedding surface plasmon resonant discs into PbSe films. Detectors utilizing a back reflector to increase the intensity of interference effects in the films showed a factor of two-times increase in responsivity over reference detectors at room temperature. Both techniques resulted in raising the operating temperature of $\mathrm{PbSe}$ devices to $290 \mathrm{~K}$. This increased operating temperature was confirmed by observing greater responsivity in devices with surface plasmon resonant disc arrays at room temperature over that of reference $\mathrm{PbSe}$ detectors at $230 \mathrm{~K}$. The same comparison was made for a PbSe detector with an embedded Pt reflector, and its performance was found to be comparable to the reference detector response at $250 \mathrm{~K}$. In this dissertation, I demonstrate that resonant structures based on either surface plasmon resonance or interference techniques increase the MWIR sensitivity of PbSe photoconductors to levels well above the current state-ofthe-art.

The details of my investigation are presented in the following order. A brief introduction to both structures is given in the remainder of this chapter. In chapter 2, the baseline characteristics of the PbSe films and reference detectors we investigated are discussed and established. In chapters 3 and 4 the theoretical background of surface plasmon resonance and thin film interference structures is provided. In chapter 5 the modeling and simulation process for selecting the parameters, as well as the predicted absorption enhancement, for resonance-enhanced $\mathrm{PbSe}$ films is presented. The 
experimental results of this investigation are presented in chapter 6 . In chapter 7, major findings are summarized, and future enhancement schemes are briefly discussed.

\subsection{Resonant Structures for Optical Management}

Resonant structures for light manipulation have been used extensively for applications such as anti-reflection coatings [24], laser cavities [25-26], and enhancement of optical detector sensitivity [27-29]. These structures typically rely on the principle of wave interference in which two waves that have a direct phase relationship occupy the same space at the same time, thereby resulting in a third wave with an amplitude that can be less than, equal to, or greater than the two incident waves [30-31]. When waves interfere they follow the superposition principle that states if there exist solutions $\mathrm{W}_{1}$ and $\mathrm{W}_{2}$ to the wave equation, described in detail in Chapter 4 , then their sum $\left(\mathrm{W}_{1}+\mathrm{W}_{2}\right)$ must also be a solution to the wave equation. The degree and type of interference can be categorized into constructive interference for additive effects, and destructive interference for resultant waves in which the amplitude is less than the combined amplitudes of the incident waves.

These interference phenomena can be observed and engineered from the macroscopic to the nanoscale. Interference cavities in which the refractive index and thickness of stacked layers are engineered to alter the transmission, reflection, or absorption of a film or substrate are examples of a macroscopic resonant structures [3233]. A relatively new class of structures that take advantage of surface plasmon resonance yields increased near-field intensity and increased scattering of the incident wave [34-35]. In this work, I investigated both the back reflector interference structure and the plasmonic disc structure. Structures were embedded into the PbSe detector films to 
increase the path length and field intensity in the PbSe layer for improved sensitivity. These structures were chosen and designed such that there would be little impact to the material composition of the PbSe film. The goal of this work was to demonstrate room temperature performance comparable to that of baseline $\mathrm{PbSe}$ detectors at lower temperatures. In the following sections, the plasmonic disc structure and back reflector interference structure are introduced.

\subsubsection{Introduction to Surface Plasmon Resonance}

To explain the losses of ballistic electrons passing through a metal, Pines and Bohm considered the quantization of coupled oscillations between free electrons in a metallic solid in 1952 [34]. Since then, this particle, which they dubbed the plasmon, has come to be known as a volume or bulk plasmon. Plasmons can be defined as coupled oscillations of free electrons in metals, as well as some dielectric materials, that can oscillate at material specific eigenfrequencies [22, 34-38].

When light interacts with a dielectric material it will do so with three primary elementary particles. The first interaction we will discuss is the exciton [12]. Consider a semiconductor medium with a bandgap energy $E_{g}$ given in joules. For a photon to be absorbed by the material, its energy must be greater than that of the bandgap,

$$
h v_{\text {photon }}=\frac{h c}{\lambda} \geq E_{g}
$$

where $\mathrm{h}$ is Planck's constant $\left(6.626 \times 10^{-34} \mathrm{JS}\right), v_{\text {photon }}$ is the frequency of the incident photon in $\mathrm{Hz}$, $\mathrm{c}$ is the speed of light in a vacuum $\left(2.998 \times 10^{8} \mathrm{~m} / \mathrm{s}\right)$, and $\lambda$ is the wavelength in $\mu \mathrm{m}$. From equation 1.3, it is evident that there is a cutoff wavelength for the photonic response for dielectric and semiconductor materials shown in equation 1.4. 


$$
\lambda_{\text {cutoff }}=\frac{h c}{E_{g}}
$$

If light at the cutoff wavelength $\lambda_{\text {cutoff }}$ is absorbed by the dielectric, an electron at the top of the valence band will then be excited to the bottom of the conduction band leaving a positively charged core called a hole. This bound electron-hole pair is called an exciton, and the interaction between a photon and an exciton is called an exciton polariton $[12,20-23]$.

Light can also couple to frequencies caused by coupled lattice vibrations in solids that manifest as excess heat [21-22]. The elementary particles of these lattice vibrations are called phonons, and, with respect to light frequencies, are categorized as either longitudinal optical or transverse optical. The interaction between a photon and phonon is called a phonon polariton.

Finally, the third interaction, which is the focus of one of the enhancement mechanisms of this investigation, is the coupling between longitudinal density fluctuations of free electrons in a metal and a photon. Defined above, these coupled density fluctuations in a metal are called plasmons. Surface plasmon polaritons (SPP) are excitations between photons and plasmons at and near the interface between a metal and a dielectric [34-38]. When light interacts with a surface plasmon, the result is an evanescent wave that decays away from the interface in both the metal and host dielectric material. These waves can either be stationary waves (localized surface plasmon polaritons) or traveling waves (surface plasmon polaritons) depending on the effective spatial dimension of the supporting structure [39]. The energy of the light must be resonant with that of the surface plasmons to create a surface plasmon polariton. 
a)

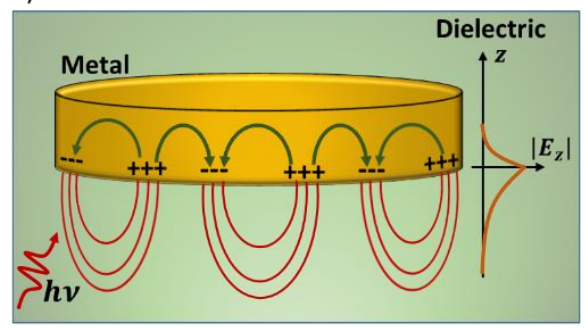

b)

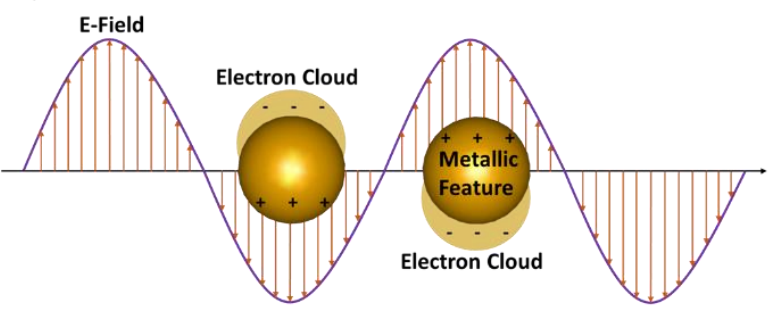

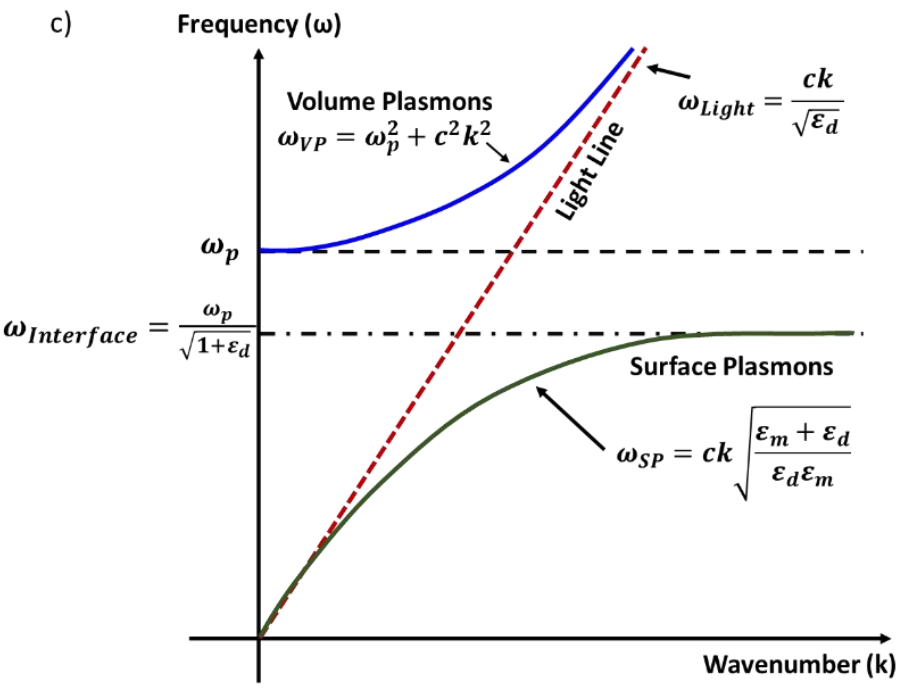

Figure 1. Depiction of a) surface plasmon polaritons at the metal/dielectric interface, b) coupled oscillations of free electrons in a metal surface feature in response to a resonant electric field, and c) surface plasmon polariton dispersion relations [34, 54].

Figure 1 contains several drawings of surface plasmon polaritons. In figure 1a surface plasmons are excited by a resonant photon generating a surface plasmon polarition, which results in an evanescent wave in both the metal and the dielectric host. Figure $1 \mathrm{~b}$ depicts the coupled oscillations of free electrons in a metallic surface feature due to a resonant electric field. Of note are the dispersion relations shown in figure 1c. The light line (red, dashed) adheres to equation 1.5, which states the angular frequency of an incident photon is related to the wavenumber $\mathrm{k}$, the speed of light $\mathrm{c}$, and is reduced by the square root of the relative permittivity $\varepsilon_{d}$ of the host dielectric material. 


$$
\omega_{\text {Light }}=\frac{c k}{\sqrt{\varepsilon_{d}}}
$$

The wavenumber of the surface plasmon, except for low frequencies, is always larger than that of the light line $[22,34]$. The wavenumber is directly proportional to energy, therefore surface plasmons, in general, have a larger energy than the light line. Recall that energy is proportional to the square of momentum, so there must be additional momentum added to an incident photon to excite a surface plasmon. This momentum mismatch can easily be observed from the solid green trace in figure $1 \mathrm{c}$, which follows the relationship of equation 1.6,

$$
\omega_{S P}=k c \sqrt{\frac{\varepsilon_{m}+\varepsilon_{d}}{\varepsilon_{m} \varepsilon_{d}}}
$$

where $\varepsilon_{m}$ is the relative permittivity of the metal. It is clear from equations 1.7 and 1.8 that one method to compensate for this momentum mismatch is to change the surrounding dielectric, thereby raising the wavenumber of the incident photon [40]. As a photon propagates through a host dielectric material, its wavenumber will be altered by the permittivity of the propagation medium. When it reaches the metal-dielectric interface, assuming such an interface exists, part of the wave is reflected. If the momentum of the photon meets the resonance condition, then an evanescent wave that decays away from the interface is created in both the host dielectric and metal structure [41]. In contrast, the solid blue trace above the light line represents the dispersion relations, shown in equation 1.7 , of volume or bulk plasmons,

$$
\omega_{V P}=\sqrt{\omega_{p}^{2}+c^{2} k^{2}}
$$

where $\omega_{p}$ is the plasma frequency of the metal. Bulk plasmons are not easily excited, because their dispersion relations lie above the light line. This is due to the large energy 
mismatch between typical photons of interest (visible-IR), and bulk plasmons. Consider the resonance condition given in equation 1.8 ,

$$
\hbar \omega_{p}=\hbar \omega
$$

where the $\hbar$ is Planck's constant $\left(6.626 \times 10^{-34} \mathrm{Js}\right)$ reduced by $2 \pi$, the left hand side is the energy of the bulk plasmon, and the right hand side is the energy of a photon. The typical energy for bulk plasmons in a metal is $\sim 10 \mathrm{eV}$, so it would take a photon in the UV to excite volume plasmons [54]. Moreover, the photons would have to propagate into the bulk of the metal, and reflection at the surface of the metal can prohibit this propagation. Therefore, in general, the resonance condition for volume plasmons in equation 1.8 is not readily met by conventional means.

Surface plasmon resonant structures offer two primary benefits of which this study takes advantage. These include increased scattering to extend the optical path length thereby increasing the absorption, and enhanced localized field intensity to increase the absorption and number of photogenerated carriers within the sensing material [35]. Indeed, gold ( $\mathrm{Au}$ ) nanodisc arrays embedded in $\mathrm{HgCdTe}$ [42] and in $\mathrm{HgSe}$ colloidal quantum dots [43] have been reported to yield up to a five-fold multiplication in detector responsivity. The plasmonic disc structure is attractive for sensor systems due to the increase in scattering cross-section, wavelength selectivity, and large increase in nearfield intensity it offers. Moreover, its simple shape can be fabricated quickly on a relatively large scale using techniques such as photolithography [38]. The surface plasmon resonant frequency red-shifts as the refractive index of the host material increases. Therefore, $\mathrm{PbSe}$ is an excellent candidate for infrared plasmonic structures, due to its large index of refraction (n 5) [44]. Typically, these structures are utilized in the 
UV and visible where the plasma frequency of metals like Au and Ag occur [45]. However, by altering either the size, morphology, metal, or host material these resonances can be shifted to the MWIR and LWIR regimes [40, 46].

Utilizing numerical and finite element techniques, discussed in Chapter 4, I designed and tailored plasmonic disc arrays into PbSe for increased MWIR sensitivity. Described in Chapter 2, the oxide layer is typically roughened using reactive ion etching (RIE) to ensure the PbSe films adhere to the Si substrate during deposition. The typical peak-to-peak roughness after this process is $500 \mathrm{~nm}$. In an effort to reduce the roughness of PbSe films, I proposed and helped develop a new process utilizing nanosphere lithography for $\mathrm{SiO}_{2}$ roughening [38]. This new process was deemed to be a necessary step to preserve the coherence properties of surface plasmon polaritons within the $\mathrm{PbSe}$ films [53]. Described in Appendix A, the nanosphere roughening process lead to a factor of five decrease in the peak-to-peak roughness in PbSe films. With help from collaborators, $\mathrm{PbSe}$ detectors with embedded Pt disc arrays were fabricated. Described in Chapter 6, I measured the material properties and detector performance of these devices, and compared them to my previous measurements, shown in Chapter 2, on $\mathrm{PbSe}$ reference detectors. I observed room temperature responsivity that was a factor of three above the reference detector under the same test conditions. Moreover, the observed room temperature responsivity in the detectors with embedded Pt disc arrays was found to be greater than that of the reference detector at $230 \mathrm{~K}$. Furthermore, I compared modeled and measured results to develop optimization and future enhancement strategies discussed in Chapter 7. 


\subsubsection{Introduction to Thin Film Interference and the Back Reflector}

Interference is of particular interest in $\mathrm{PbSe}$ thin films, due to the presence of multiple layers with varying indices of refraction which will be described in Chapters 2 and Chapter 4. The index of refraction of PbSe was stated above without derivation. However, to understand the effects on an incident wave as it propagates through the film, it is important to understand where the index comes from, as well as how it relates to other film properties. Described in Chapter 2, the films we investigated were composed of three semiconductor layers on top of a Si substrate. These layers, in order from the bottom, are $\mathrm{SiO}_{2}, \mathrm{PbSe}$, and $\mathrm{PbI}_{2}$, and each is on the order of $300 \mathrm{~nm}$ to $1 \mu \mathrm{m}$ thick depending on process conditions. The substrate, by comparison, is much larger having a thickness of approximately $250-450 \mu \mathrm{m}$. Due to the large substrate thickness and subwavelength thickness of the layers, it can be assumed that the constituent parts of an incident beam of light that are reflected or transmitted have a direct phase relationship and are coherent with respect to one another [30]. Further, due to the fact that the wavelength of incident light in the infrared regime is much larger than the interatomic spacing between the atoms that make up the films the interaction of incident light with the films can be assumed to span many unit cells, allowing the use of a macroscopic treatment. The derivation of interference within thin films will be discussed in Chapter 4, however, it is important to briefly describe the benefits of incorporating an artificial structure within $\mathrm{PbSe}$ to enhance sensitivity.

When light propagates from a lower to a higher refractive index medium, the waves will undergo a phase shift of 180 degrees upon reflection [47]. The transmitted wave will be refracted according to Snell's law [30]. When the beam travels from a lower 
index, such as air or vacuum, to a higher index, such as $\mathrm{PbI}_{2}$ or $\mathrm{PbSe}$, the light will bend toward the normal at the refracted angle. This refracted angle becomes the angle of incidence for the second interface, where part of the light will again be reflected and part will be transmitted.

If the refractive index of the medium immediately following the second interface is lower than that of the preceding medium, then the wave will retain its current phase upon reflection. The reflected beam will then propagate through the medium a second time, and refract bending at an angle away from the normal as it exits the film. In fact, the beam that emerges will do so at the same angle as the first reflected wave (this is also true of the incident and transmitted angle). Now, the first reflected wave, which has undergone a $\pi$ phase shift, and the second reflected wave can interfere resulting in an intensity that deviates from the intensities of the original waves.

Consider the representation of the multilayer films depicted in figure 2. For now, we will only discuss those light beams that undergo normal path deviation in an ideal thin film, so the roughness of the PbSe film will be ignored. This assumption will be true for at least part of the light beam, even in rough films, as it will propagate through the film without deviation. Let the initial phase of the light as it passes from air to the $\mathrm{PbI}_{2}$ layer be regarded as 0 radians. At the air- $\mathrm{PbI}_{2}$ interface, part of the light will be reflected and part of the light will be transmitted. The amount of light reflected and transmitted can be calculated by using the Fresnel equation, shown in equation 1.9, to calculate the reflectance [48].

$$
R=\left|\frac{n_{0}-n_{1}}{n_{0}+n_{1}}\right|^{2}
$$




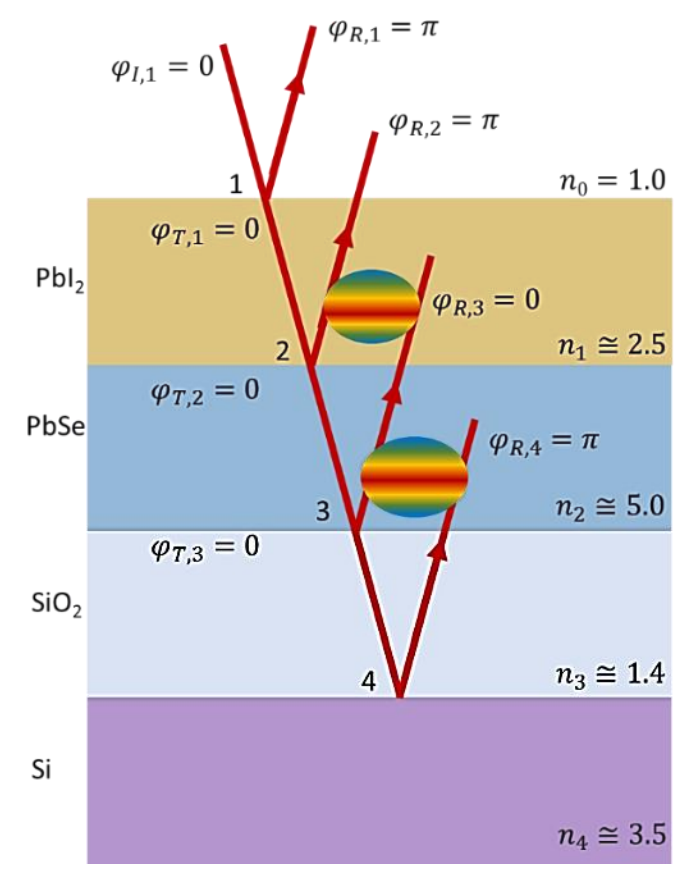

Figure 2. Interference in idealized PbSe thin films on Si substrate.

Assuming the interfaces are non-absorbing, the transmittance can be found by taking the difference between 1 and the calculated reflectance. Therefore, at the air- $\mathrm{PbI}_{2}$ interface, $18 \%$ of the light will be reflected and $82 \%$ will be transmitted. Upon reflection, there will be a $\pi$ radian phase shift due to $\mathrm{PbI}_{2}$ having a larger refractive index than air. The transmitted beam as it approaches the $\mathrm{PbI}_{2}-\mathrm{PbSe}$ interface will also undergo a phase shift upon reflection, with approximately $11 \%$ of the light being reflected from the interface. This reflected ray upon exiting the $\mathrm{PbI}_{2}$ layer has the ability to constructively or destructively interfere with the first reflected ray. This pattern will repeat through the film, with the reflectances of the $\mathrm{PbSe}-\mathrm{SiO}_{2}$ interface and the $\mathrm{SiO}_{2}-\mathrm{Si}$ interface being $32 \%$ and $18 \%$, respectively. There will be another $\pi$ phase shift at the $\mathrm{Si}_{-} \mathrm{SiO}_{2}$ interface, which will interfere with the reflected ray in the PbSe layer. Further, the reflected ray in the $\mathrm{PbI}_{2}$ layer can also interfere with the reflected ray from the PbSe layer. Described in detail in Chapter 4, it is clear from the Fresnel reflections and the existing phase shifts 
that the layer thicknesses can be tuned such that a significant portion of the light has a distinct probability of interference, resulting in increased field intensity in the PbSe layer.

Figure 2 illustrates the regions in which interference can occur in baseline $\mathrm{PbSe}$ films. However, there are two limitations with respect to interference enhancement in baseline PbSe films. First, the layers need to be engineered to specific thicknesses to fully realize the enhancement. The chemical bath deposition process, though repeatable on the whole, has wide variation from deposition to deposition that can lead to inhomogeneity in layer thickness and composition $[9,11]$. Furthermore, the sensitization process lacks the precision for optically precise tailoring of layer thickness. The second limitation is the amount of light that is available to reflect back into the film for interference. Described in chapter 2, the PbSe films investigated herein have an absorption of $\sim 30 \%$. The reflectance at each layer, shown in table 1, is a fraction of the light incident at each interface. Considering the absorption in PbSe and the reflectance at each interface, $23 \%$ of light would be lost to the substrate. Therefore, the resultant intensity increase from constructive interference can be weak.

Table 1. Calculated interface reflectance and reflection-induced phase shifts in investigated PbSe films.

\begin{tabular}{|c|c|c|}
\hline PbSe Film Interface & Reflectance & Phase Shift \\
\hline Air-PbI & $18 \%$ & $\pi$ \\
\hline $\mathrm{PbI}_{2}-\mathrm{PbSe}$ & $11 \%$ & $\pi$ \\
\hline $\mathrm{PbSe}_{-} \mathrm{SiO}{ }_{2}$ & $32 \%$ & 0 \\
\hline $\mathrm{SiO}_{2}-\mathrm{Si}$ & $18 \%$ & $\pi$ \\
\hline
\end{tabular}

To address these shortcomings, I have explored the addition of an embedded metallic reflector, which will be referred to as a back reflector. The back reflector is attractive as a PbSe enhancement mechanism due to its ease of fabrication, wide area coverage, wavelength selectivity, and large increase in absorption and field intensity [49- 
51]. The difference in the PbSe structure with the embedded metallic reflector is minimal. The metal reflector, in this case Pt, is located on top of the substrate, with the remaining layers having their same order on top of the reflector. $\mathrm{The}^{\mathrm{SiO}_{2}}$ layer serves two purposes in this configuration. The first is similar to that of the baseline film in that it insulates the underlying layer from $\mathrm{PbSe}$, and the second is as a dielectric spacer with which to change the thickness of the film to selectively tune the resonant enhancement $[9,52]$. Stated above, approximately $23 \%$ of an incident light beam would transmitted to the substrate after the PbSe film. With a metallic reflector it is possible for all of the light that propagates through the $\mathrm{PbSe}$ film to be reflected back into the active layer resulting in increased intensity of constructive interference effects.

To investigate the effect of this increased interference intensity on the sensitivity of $\mathrm{PbSe}$, I have designed and tailored Pt back reflector films for increased MWIR sensitivity using numerical and finite element methods. Assuming smooth films and sharp interfaces, the back reflector sets up a plane-parallel cavity within the PbSe film. However, the rough nature of these $\mathrm{PbSe}$ films causes light to scatter randomly as it propagates through them. This scattering can affect the optical path length within the films, and reduce the effectiveness of the cavity. The nanosphere lithography process, described in Appendix A, for roughening the $\mathrm{SiO}_{2}$ was also used here to prepare $\mathrm{PbSe}$ substrates for the back reflector structure. With help from collaborators, PbSe films with an embedded Pt back reflector were deposited and fabricated into photoconductive devices. Described in Chapter 6, I measured the detector performance of these devices, and compared them to measurements I made previously on the PbSe reference detector. I observed room temperature responsivity that was a factor of two above the reference 
detector under the same test conditions. Furthermore, two distinguishable peaks were observed, and confirmed to be the result of constructive interference within the $\mathrm{PbSe}$ film. I also observed the room temperature responsivity in detectors with an embedded Pt back reflector to be comparable to the reference detector at $250 \mathrm{~K}$. Modeled and measured results were compared to develop optimization and future enhancement strategies discussed in Chapter 7. 


\section{Chapter 1 References}

[1] J. C. Leachtenauer, and R. G. Driggers, Surveillance and reconnaissance imaging systems: Modeling and Performance Prediction. Norwood, MA: Artech House, Inc, 2004.

[2] Spectral Sciences, Inc. [online] Available: http://modtran.spectral.com/modtran_home\#plot. [Accessed Sept. 11, 2018]

[3] K. Kasunic, Optical Systems Engineering. McGraw-Hill Education, 2011.

[4] C. J. Willers, Electro-Optical Systems Analysis and Design, Bellingham, WA: SPIE Press, 2013.

[5] D. A. Kondas, "Introduction to Lead Salt Infrared Detectors," Technical Report ARFSD-TR-92024, US Army Armament Research, Development, and Engineering Center, 1993.

[6] G. Vergara, et al. "Polycrystalline lead selenide: the resurgence of an old infrared detector,” Opto-Electronics Review, 15(2), 110-117, 2007.

[7] D. J. Lovell, "Development of Lead Salt Detectors." American Journal of Physics 37, 467, 1969.

[8] Thanikaikarsan, et al. "Growth and Characterization of Lead Selenide Thin Films." J. Mater. Electron. 23, 1562-1568, 2012.

[9] S. -S., Yoo, et al. "High Operating Temperature MWIR Photodetecotrs Development Using Polycrystalline PbSe Technology," presented at The U.S. Workshop on the Physics and Chemistry of II-VI Materials, Chicago, IL, 2017.

[10] J. Phillips, "Evaluation of the Fundamental Properties of Quantum Dot Infrared Detectors.” Journal of Applied Physics, 91 (7), 4590-4594, 2002. 
[11] M. -H., Jang, "Effect of Sensitization on PbSe IR Detector Materials Properties." Manuscript submitted to J. of Elect. Mat., 2017.

[12] D. A. Neamen, Semiconductor Physics and Devices: Basic Principles, $3^{\text {rd }}$ Ed, New York, NY: McGraw Hill, 2003.

[13] J. N. Humphrey and R. I. Petritz, "Photoconductivity of Lead Selenide: Theory of the Mechanism of Sensitization." Physical Review, vol 105, no 6, 1957.

[14] P. Kuman, et. al. "PbSe Mid-IR Photoconductive Thin Films (Part 1): Phase Analysis of the Functional Layer." Journal of Alloys and Compounds, 724, 316326, 2017.

[15] Lihuazhao, et al. "Understanding Sensitization Behavior of Lead Selenide Photoconductive Detectors by Charge Separation Model.” Journal of Applied Physics, 115, 084502, 2014.

[16] P. R. Norton, "Photodetectors," in McGraw-Hill's Handbook of Optics, 3rd ed., Vol. 2, New York: McGraw-Hill, 2010, pp. 24.3-24.100.

[17] E. Hergert, "Detectors: Guideposts on the Road to Selection," in the Photonics Directory, 5nd Ed. Laurin Publishing, 2006.

[18] R. A. Smith, "Detectors for Ultraviolet, Visible, and Infrared Radiation," Applied Optics, Vol. 4, No. 6, 1965.

[19] A. Rogalski, M. Kopytko, and P. Martyniuk, Antimonide-based Infrared Detectors: A New Perspective, Bellingham, WA: SPIE Press, 2018.

[20] R. A. Serway, C. J. Moses, and C. A. Moyer, "The Solid State," in Modern Physics, $3^{\text {rd }}$ ed, Belmont, CA: Borrks/Cole-Thomson Learning, 2005. 
[21] C. Kittel, Introduction to Solid State Physics, Hoboken, NJ: John Wiley and Sons, Inc. 2005.

[22] M. P. Marder. Condensed Matter Physics, $2^{\text {nd }}$ ed. Hoboken, NJ: John Wiley and Sons, Inc. 2010.

[23] D. J. Lovell, "The Development of Lead Salt Detectors," American Journal of Physics, Vo. 37, No. 2, 1957.

[24] K. Rabinovitch and A. Pagis, "Multilayer antireflection coatings: theoretical model and design parameters," Applied Optics, Vol. 14, No. 6, 1975.

[25] W. T. Silfvast, Laser Fundamentals, New York, NY: Cambridge University Press, 2000.

[26] C. C. Davis, Lasers and Electro-Optics: Fundamentals and Engineering, $2^{\text {nd }}$ ed. New York, NY: Cambridge University Press, 2014.

[27] K. K. Choi, et al. "Electromagnetic modeling and resonant detectors and arrays." Infrared Physics \& Technology 70, 2015.

[28] K. K. Choi, et al. "Resonant structures for infrared detection." Applied Optics, Vol. 56, No. 3, 2017.

[29] K. K. Choi, et al. "Resonator-quantum well infrared photodetectors." Appl. Phys. Lett. 103, 201113, .2013.

[30] E. Hecht, Optics, $4^{\text {th }}$ ed. New York: Addison-Wesley, 2002.

[31] R. E. Fischer, et al. Optical Systems Design, $2^{\text {nd }}$ ed. SPIE Press, McGraw Hill, 2008. 
[32] M. S. Rauscher, et al. "Dual-channel mid-infrared sensor based on tunable FabryPerot filters for fluid monitoring applications." Sensors and Actuators B, 259, 2018.

[33] E. Gayo and J. Frutos, "Interference filters as an enhancement tool for infrared thermography in humidity studies of building elements." Infrared Physics and Technology 38, 1997.

[34] D. Sarid and W. Callner, Modern Introduction to Surface Plasmons: Theory, Mathematical Modeling, and Applications. New York, NY: Cambridge University Press, 2010.

[35] J. Alda and G. Boremann. Infrared Antennas and Resonant Structures, Billingham, WA: SPIE Press, 2017.

[36] A. A. Toropov and T. V. Shubina, Plasmonic Effects in Metal-Semiconductor Nanostructures. Great Clarendon, Oxford, United Kingdom: Oxford University Press, 2015.

[37] E. Li and H. Chu, Plasmonic Nanoelectronics and Sensing. United Kingdom: Cambridge Press, 2014.

[38] J. Jahns and S. Helfer, Introduction to Micro- and Nanooptics. Hoboken, NJ: John Wiley and Sons, Inc., 2012.

[39] W. A. Murray and W.L. Barnes, "Plasmonic Materials." Adv. Mater. 19, 37713782, 2007.

[40] Z Jaks ̌rc', et al. "Red Shifting Approach for Nanoplasmonic Enhancement of Semiconductor Infrared Detectors." Proc. 28th International Conference on Microelectronics, Serbia, 2012. 
[41] H. N. Daghestani and B. W. Day, "Theory and applications of Surface Plasmon Resonance, Resonant Mirror, Resonant Waveguide Grating, and Dual Polarization Interferometry Biosensors,” Sensors, 10, 9630-9646, 2010.

[42] J. Liang, et al. "Modeling of $\mathrm{HgCdTe}$ photoconductive infrared detector with metallic nanostructures.” IEEE, NUSOD, 2014.

[43] X. Tang, et al. "Plasmon resonance enhanced colloidal HgSe quantum dot filterless narrowband photodetectors for mid-wave infrared." J. Mater. Chem. C, 5, 362-369, 2017.

[44] G. Bauer and H. Krenn, "Lead Selenide (PbSe)," in Palik, E.D. Editor, Handbook of Optical Constants of Solids, (Vol. 1, pp. 517 -523). San Diego, CA. United States: Academic Press, Inc. 1985.

[45] A. J. Wood, "Fabrication and Characterization of a Plasmonic Grating Platform for Enhanced Fluorescence Biosensor Applications,” PhD Thesis, University of Missouri, Columbia, MO, 2016.

[46] D. W. Peters, et al. "Plasmonic Nanoantennas for Enhanced Midwave and Longwave Infrared Imaging," Proc. Of SPIE, Vol. 9467, 2015.

[47] OP-Tec: National Center for Optics and Photonics Education, Fundamental of Light and Lasers, 2nd ed. Texas: Op-TEC, 2013.

[48] Balanis, C.A. Advanced Engineering Electrodynamics, 2nd Edition. Wiley, 2012.

[49] S. Jain, et al. "Broadband absorption enhancement in ultra-thin crystalline Si solar cells by incorporating metallic and dielectric nanostructures in a back reflector," Pro. Photovolt: Res. Appl., Vol. 23, 2015. 
[50] J. Piotrowski and A. Piotrowski, "Uncooled infrared photodetectors in Poland," Opto-Electron. Rev., 14, no. 1, 2006.

[51] J. J. Talghader, A. S. Gawarikar, and P. R. Shea, "Spectral selectivity in infrared thermal detection," Light: Science \& Applications, Vol. 1, 2012.

[52] X. Tang, M. Ackerman, and P. Guyot-Sionnest, "Thermal Imaging with Plasmon Enhanced HgTe Collidal Quantum Dots Photovoltaic Devices," manuscript nn2018-03871g submitted to ACS Nano, 2018.

[53] G. A. Farias and A. A. Maradudin, "Effect of surface roughness on the attenuation of surface polaritons on metal films," Phys. Rev. B 27, 1983.

[54] V. M. Shalaev, "Introduction to Metal Optics," Lecture from ECE 695 at Purdue University, 2006. [Online]. Available: https://nanohub.org/resources/1852/download/2006.10.05-ece695s-109.pdf. [Accessed: Sept. 25, 2017]. 


\section{Chapter 2: Lead Selenide (PbSe) as a Detector Material}

PbSe has been studied extensively since WWII for military applications. German scientists were very interested in the military applications of infrared technology, pursuing with intense fervor lead-salt technology [48-49]. Research Efforts in Germany, led by Edgar W. Kutzcher, focused on lead sulfide (PbS). Kutzcher observed high SWIR sensitivity in $\mathrm{PbS}$ crystallites, which were capable of bolometer caliber operation. His work would lead to passive IR detectors employed by Germany during WWII for aircraft and ship detection. These detectors were a leap forward in military technology, making the invisible IR spectrum a tool to be exploited. To extend the spectral sensitivity range of PbS, which was limited to $1 \mu \mathrm{m}-3.3 \mu \mathrm{m}$, PbSe was investigated by German scientists leading to appreciable sensitivity at $77 \mathrm{~K}$. The development of PbSe detectors opened up the IR spectrum, spanning the SWIR to MWIR and beyond, with spectral sensitivity from $1 \mu \mathrm{m}-7 \mu \mathrm{m}$. In parallel to German efforts, British scientists reported excellent sensitivity in $\mathrm{PbSe}$; not only at cryogenic temperatures, but at room temperature with a cutoff up to $5 \mu \mathrm{m}$ [49]. In the early 1940's, the United States in parallel started to take interest in $\mathrm{Pb}$ salt based infrared technology. The National Defense Research Committee (NDRC) was formed with the express purpose of developing and understanding infrared technology. Spearheaded by Robert J Cashman, efforts were focused on PbS photoconductive cells [48]. Cashman was a pioneer in his field, developing and optimizing chemical deposition techniques for $\mathrm{Pb}$-salt synthesis. He discovered that the sensitivity of $\mathrm{PbS}$ could be enhanced by synthesizing films in an oxygen rich atmosphere. It should be noted that this was a precursor to the current sensitization process used for $\mathrm{Pb}$-salts [12]. 
Immediately following WWII, military interest in $\mathrm{Pb}$-salts saw a decline, with research efforts being focused more on optimizing the chemical synthesis and understanding the material characteristics than on device improvement. In 1947, however, the US Navy contracted with the Eastman Kodak company to develop PbS cells as a result of their successful demonstration of long range detection of the thermal signature of the Dove missile, an air-to-air ordnance [49]. Around the same time, Henry Levinston and his graduate students at Syracuse University were investigating the photoconductive nature of $\mathrm{PbSe}$ and $\mathrm{PbTe}$. Starting in 1948, the Naval Ordnance Laboratory (NOL) took on a decade long charge of determining the photoconductive and material nature of PbSe and PbS [48-49]. Between 1960 and 1980, new, more expensive technologies caused a decline in $\mathrm{Pb}$-salt research, and, by the mid-1980s, $\mathrm{Pb}$-salts had taken a back seat to compounds like InSb and $\mathrm{HgCdTe}$ [49-50]. As mentioned above, these materials typically offer greater infrared sensitivity at the cost of added system complexity and cryogenic operating temperatures. Seeing a resurgence in recent years, $\mathrm{PbSe}$ is attractive for both military and space-based applications due to its low-cost and high-temperature operation over the 3-5 $\mu \mathrm{m}$ atmospheric window.

The PbSe films discussed herein are comprised of three semiconductor layers grown on single side polished silicon $(\mathrm{Si})$ substrates. First, a $0.5-1.5 \mu \mathrm{m}$ thick silicondioxide $\left(\mathrm{SiO}_{2}\right)$ layer is grown on top of the $\mathrm{Si}$ substrate by plasma enhanced chemical vapor deposition (PE-CVD). Reactive ion etching (RIE) is used to etch the $\mathrm{SiO}_{2}$ layer to a surface roughness of approximately $500 \mathrm{~nm}$ peak-to-peak. This roughening step is necessary for adequate adhesion of $\mathrm{PbSe}$ to the substrate during deposition. A process based on chemical bath deposition methods is then used to synthesize lead acetate 
trihydrate $\left(\mathrm{Pb}\left(\mathrm{C}_{2} \mathrm{H}_{3} \mathrm{O}_{2}\right)_{2}\right)$ and optical grade selenourea $\left(\left(\mathrm{H}_{2} \mathrm{~N}\right)_{2} \mathrm{C}=\mathrm{Se}\right)$ into a PbSe film with an approximate thickness of $1 \mu \mathrm{m}$. The PbSe films then undergo sensitization by oxidation and halogenation at $420{ }^{\circ} \mathrm{C}$ and $350{ }^{\circ} \mathrm{C}$, respectively. The halogen used for sensitization for these films is iodine, thereby converting approximately half of the $\mathrm{PbSe}$ film into lead iodide $\left(\mathrm{PbI}_{2}\right)$ [1-2].

\subsection{PbSe Crystal Structure}

To begin the discussion of PbSe films, let the film during the various processes of synthesis be identified by the monikers "as-grown", "oxidized", and "sensitized". Samples of the film from various processes are shown in figure 3a. The as-grown $\mathrm{PbSe}$ looks grey in color, while oxidized $\mathrm{PbSe}$ has a blue hue. When the sensitization process leads to a uniform conversion, the $\mathrm{PbI}_{2}$ has a slight yellow tint. If the film is over iodized then the top film will look completely yellow, and can lead to decreased sensitization.

Depicted in figure 3b, the films consist of three layers on top of a Si substrate, with a total film thickness, including the $\mathrm{SiO}_{2}$ layer, of $\sim 2 \mu \mathrm{m}$. The films are polycrystalline in nature, so the atoms within crystallites are arranged periodically, while the areas between grains show very little order [13].
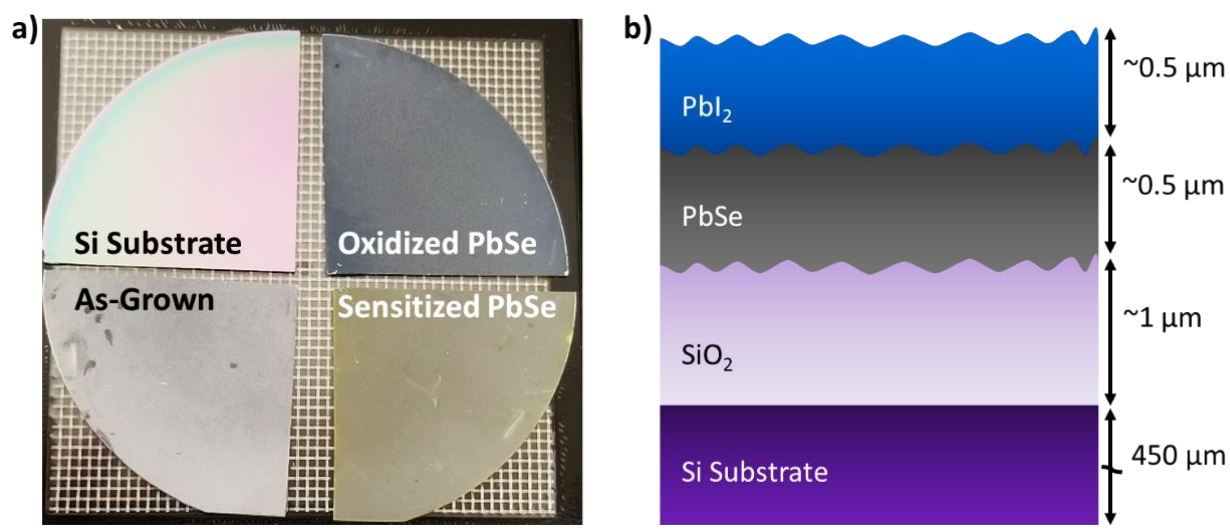

Figure 3. PbSe films grown by chemical bath deposition for this investigation; a) Shown from left to right are the Si substrate (top-left), as-grown PbSe film (bottom-left), oxidized PbSe film (top-right), and sensitized PbSe film (bottom-left); b) Depiction of sensitized PbSe film cross-section. 
An energy dispersion spectroscopy (EDS) map [3] collected during our early studies of the sensitized PbSe film is shown in figure 4 [1]. The green points in the top image indicate the presence of $\mathrm{Pb}$ throughout the film, as expected. The other colored points represent Se (magenta), oxygen (yellow), iodine (cyan), and carbon (red). The bottom of the figure contains point clouds representing the density and location of each secondary compound. As expected, the Se and I portions are on top of each other, representing the interface between $\mathrm{PbSe}$ and $\mathrm{PbI}_{2}$. This interface is semi-sharp, with gradients of each compound permeating into the opposite layer. The oxygen seen in the bottom of the film is associated with the $\mathrm{SiO}_{2}$ layer beneath the $\mathrm{PbSe}$. Oxygen is also seen throughout the film, which is where the PbSe has been converted to oxygenated-lead compounds during oxidation [2]. Interestingly, a large amount of carbon is found throughout the film. These carbon molecules play a crucial role in the $\mathrm{PbSe}$ sensitization process.

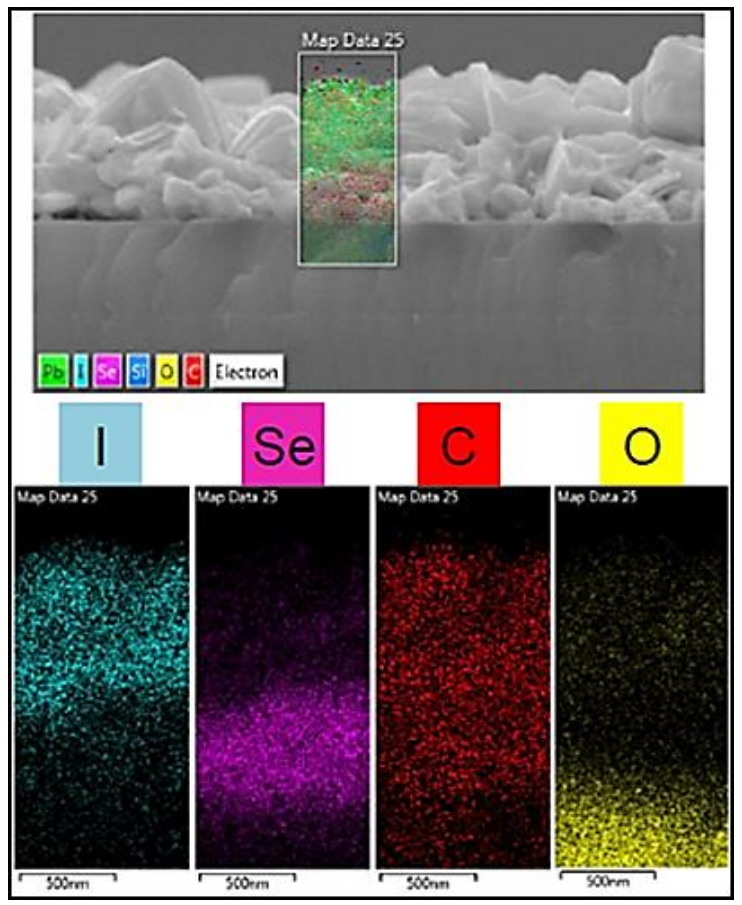

Figure 4. EDS map depicting the polycrystalline nature of, as well as the interface between, the PbSe and $\mathrm{PbI}_{2}$ within the film after sensitization [1]. 
During the synthesis process, the crystal structure of PbSe thin films undergoes various changes. Jang, et al. used x-ray diffraction to show that the films start out with a polycrystalline face-centered cubic (FCC) phase, while the oxidized films show evidence of the conversion of part of the film to monoclinic or orthorhombic $\mathrm{PbO}_{2}\left(\mathrm{SeO}_{3}\right)$ depending on the oxidation time [2]. After iodine halogenation, the $\mathrm{PbI}_{2}$ layer contains a polytype 12R crystal structure [4]. Figure 5 depicts the different crystalline structures contained within the films.

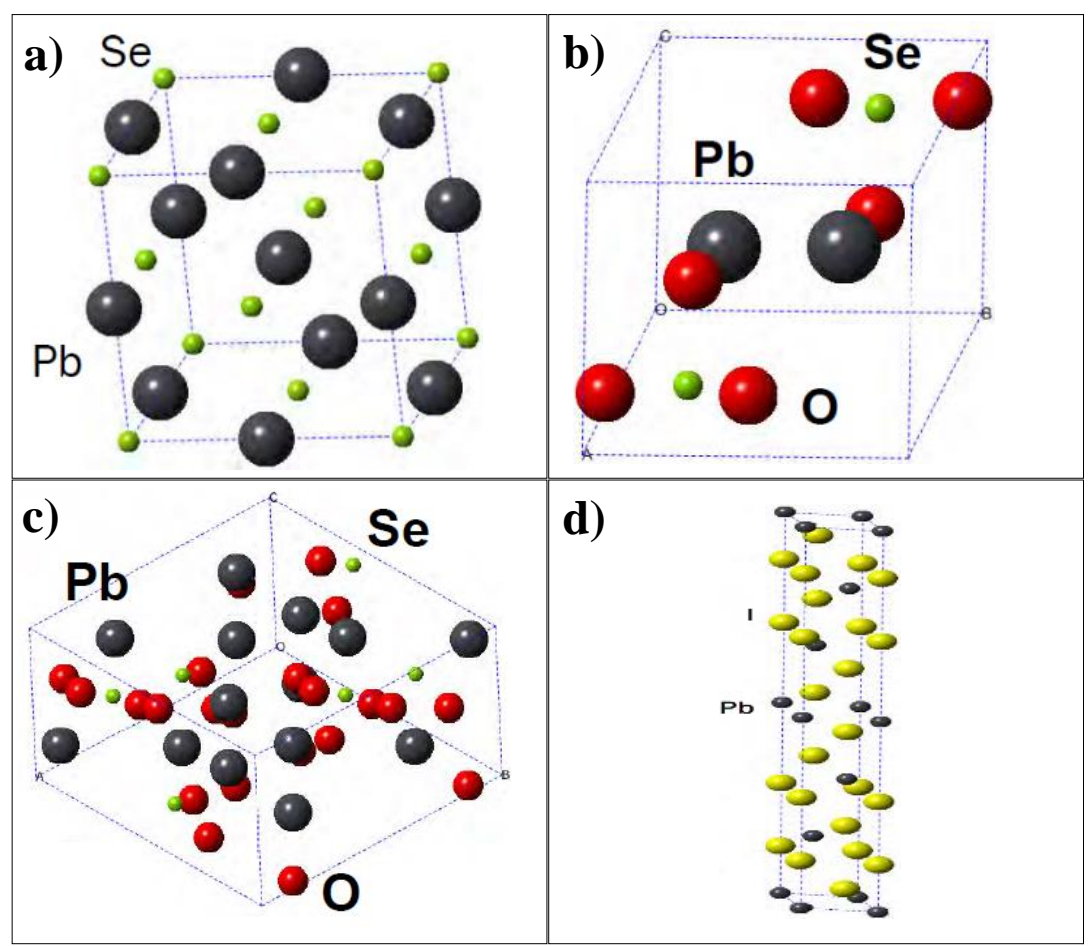

Figure 5. Depiction of crystal structure of PbSe films; a) as-grown $\mathrm{PbSe}, b)$ monoclinic $\mathrm{Pb}_{3} \mathrm{O}_{2}\left(\mathrm{SeO}_{3}\right), c$ ) orthorhombic $\mathrm{Pb}_{3} \mathrm{O}_{2}\left(\mathrm{SeO}_{3}\right)$, d) polytype 12R $\mathrm{PbI}_{2}$ [2].

We collected secondary electron images (SEI), shown in figure 6, on the oxidized and sensitized PbSe films during this investigation using scanning electron microscopy (SEM). From figure $6 \mathrm{a}$, the grain size of PbSe crystallites range from $\sim 0.3-1 \mu \mathrm{m}$. It would seem from figure $6 \mathrm{~b}$ that the crystallite size increases to $0.5-3 \mu \mathrm{m}$ after the sensitization step. However, as reported in [5] and [6] for similar PbSe films, the grain 
size tends to decrease while the agglomeration of microcrystallites increases during the iodine sensitization process.

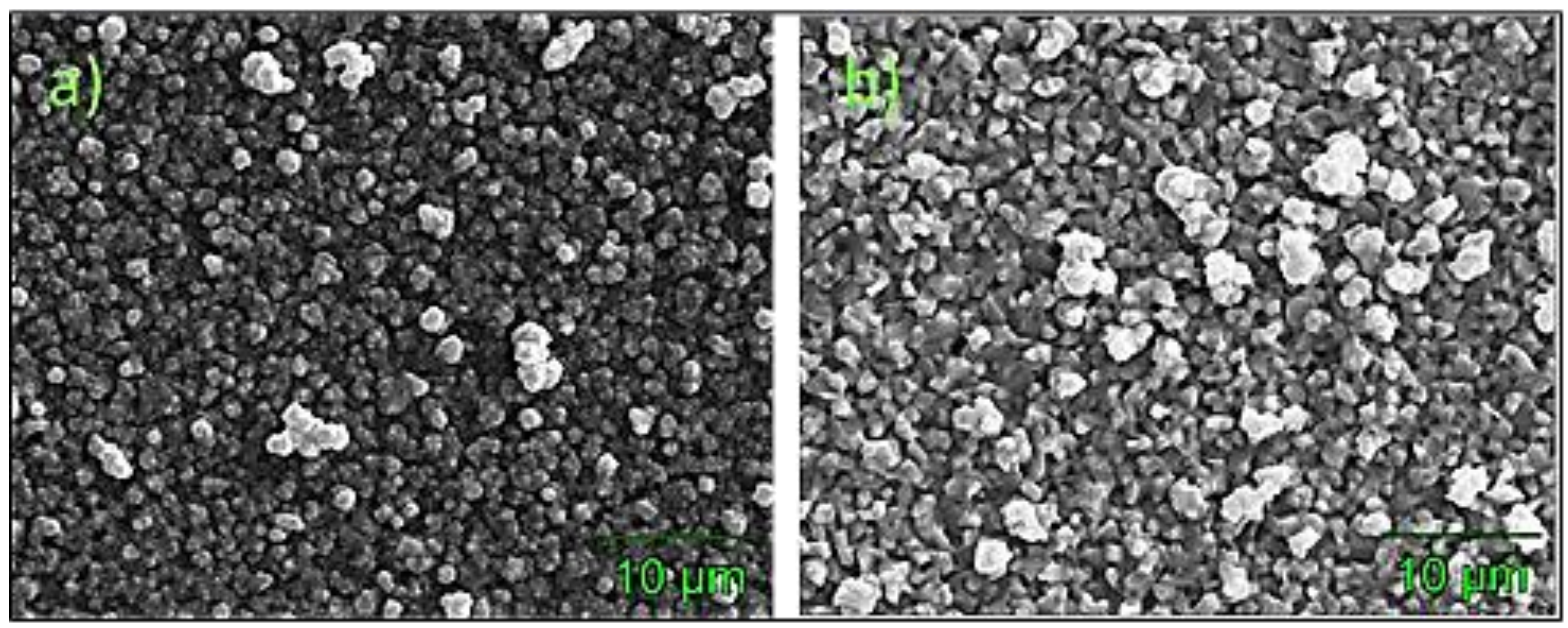

Figure 6. Secondary electron images obtained using a scanning electron microscope on the of a) oxidized, and b) sensitized PbSe films of this study. During the iodine sensitization process, crystallite size is reduced while the number and size of crystallite agglomerations increases.

High resolution transmission electron microscope (HRTEM) images were also collected by our collaborators from the same sensitized PbSe films. Figure 7a shows agglomerations larger than $500 \mathrm{~nm}$, reinforcing the SEI results. Interestingly, a carbon shell is observed in figure $7 \mathrm{~b}$ and $7 \mathrm{c}$, which has an oxidation temperature-dependent thickness of 10-20 nm and surrounds both the $\mathrm{PbSe}$ and $\mathrm{PbI}_{2}$ crystallites [2]. The carbon shell is formed by organic precursors in the lead acetate, which are incorporated during the deposition process. This carbon shell may contribute to photoconduction by either the barrier or charge separation models [7-8]. Carriers may move from the PbSe grains to the carbon shell, which acts as a thin conductive layer making it necessary for high sensitivity PbSe detectors. 

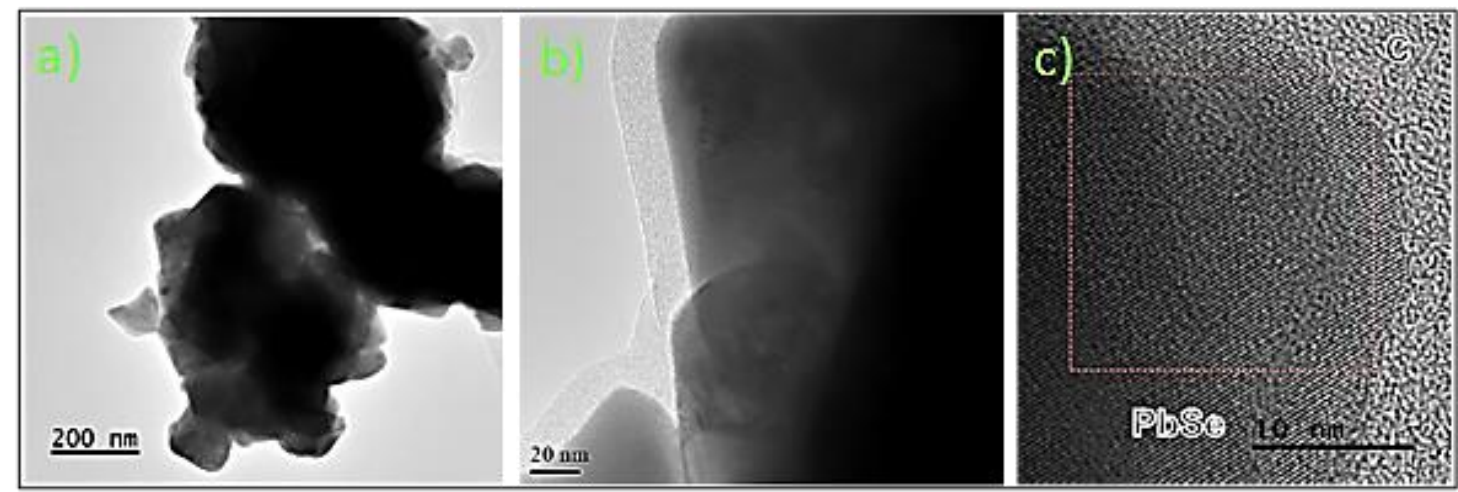

Figure 7. HRTEM images collected on sensitized PbSe films of this study. a) PbSe crystallite agglomerations after sensitization, b) carbon shell surrounding PbSe crystallite for $400{ }^{\circ} \mathrm{C}$ oxidation, and c) reduced carbon shell after $420^{\circ} \mathrm{C}$ oxidation.
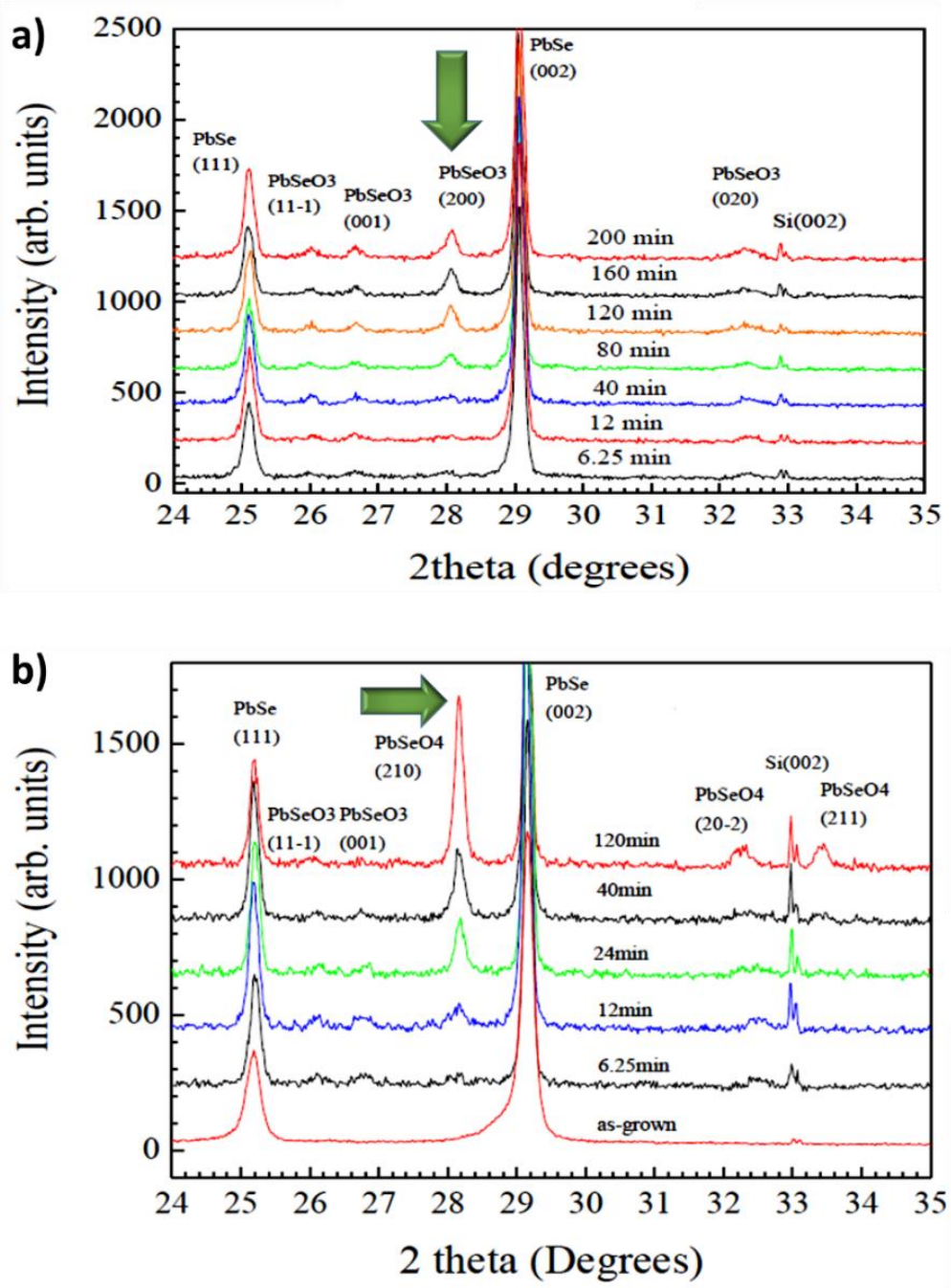

Figure 8. The XRD spectra of oxidized PbSe films as a function of time and temperature [2, 9]. When the oxidization temperature is increased from a) $400{ }^{\circ} \mathrm{C}$ to b) $460{ }^{\circ} \mathrm{C}$, the $\mathrm{PbSeO}_{3}$ compound is converted to $\mathrm{PbSeO}_{4}$. Longer times can lead to over oxidation, and decreased film sensitivity. 
Another function this carbon layer serves is to protect the crystallites from over oxidation during the sensitization process $[2,9]$. X-ray diffraction (XRD) spectra were collected on these films by our collaborators as a function of oxidation time and temperature [2]. As the oxidation temperature increases the $\mathrm{PbSeO}_{3}$ peak becomes $\mathrm{PbSeO}_{4}$, and the carbon shell shrinks $[2,9]$. The conversion can be seen in figure 8 when comparing samples oxidized at $400{ }^{\circ} \mathrm{C}$ (top) and $460{ }^{\circ} \mathrm{C}$ (bottom). It is also evident from figure 8 that the oxidation time is an important factor in determining the amount of incorporated oxygen in the film. As the time increases from 6.25-200 min, both the $\mathrm{PbSeO}_{3}$ and $\mathrm{PbSeO}_{4}$ peaks grow significantly. This increase in peak amplitude can have a detrimental effect on the sensitivity of the PbSe films, emphasizing the need for the optimization and control of sensitization conditions [9].

\subsection{Photoconduction in PbSe}

To first approximation, the band structure of these PbSe thin films can be found using the electron affinity rule [10]. The PbSe has a bandgap of $0.29 \mathrm{eV}$, while $\mathrm{PbI}_{2}$ has a bandgap of $2.41 \mathrm{eV}[7,11]$. Furthermore, shown below, PbSe is an n-type material and $\mathrm{PbI}_{2}$ p-type [12]. $\mathrm{PbSe}$ and $\mathrm{PbI}_{2}$ conduction bands are both considered to be parallel to the vacuum level. Moreover, the straddling case for heterojunctions is assumed. Using this configuration, the electron affinity, defined as the energy difference between the bottom of the conduction band and the vacuum level [12], for PbSe $\chi_{P b S e}$ is greater than that of $\mathrm{PbI}_{2} \chi_{\mathrm{PbI}_{2}}$. The electron affinity for both materials can be used to find the difference in the conduction band energies by equation 2.1 .

$$
\Delta E_{c}=e\left(\chi_{P b S e}-\chi_{P b I 2}\right)
$$


The valence band offset, shown in equation 2.2 , can be found using the difference of the bandgap energies and equation 2.1 .

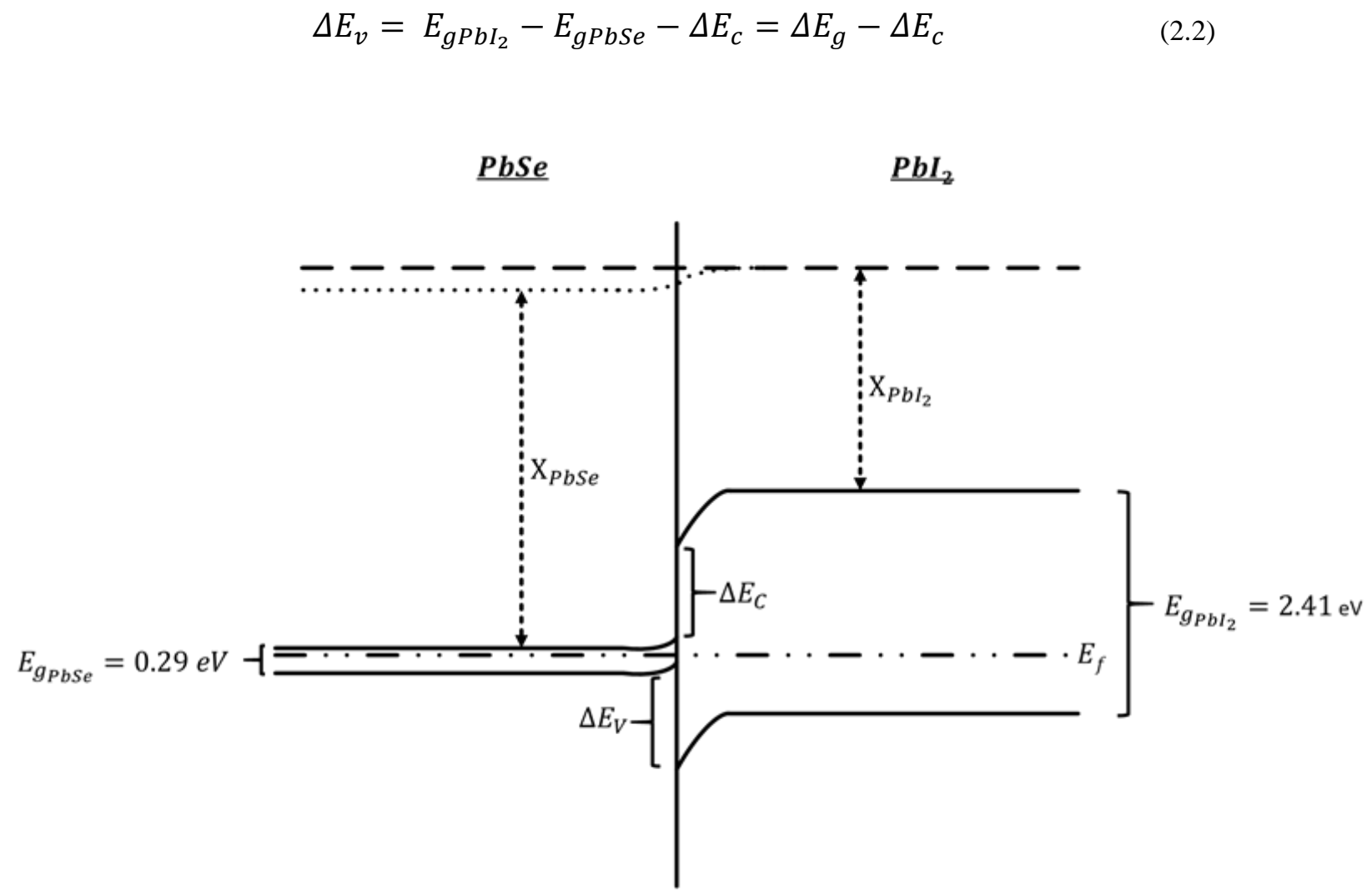

Figure 9. Qualitative band diagram sketch of PbSe photoconductive films obtained using the electron affinity rule. When PbSe and $\mathrm{PbI}_{2}$ form an interface, the Fermi-level of the system lowers enabling increased hole flow.

When these two materials are brought together, the Fermi-levels align and electrons from $\mathrm{PbSe}$ will flow into $\mathrm{PbI}_{2}$, while at the same time holes from $\mathrm{PbI}_{2}$ will flow into $\mathrm{PbSe}$ creating a space-charge or depletion region [12-13]. Band bending occurs at the junction, which is a result of the change in the vacuum level due to the alignment of the Fermi-levels. Figure 9 shows the author's qualitative drawing of the band structure in PbSe films constructed by using the band gaps of $\mathrm{PbSe}$ and $\mathrm{PbI}_{2}$, as well as equations 2.1 and 2.2. It should be noted that the PbSe films that were grown for this study are 
polycrystalline and porous, so the interface may not be as sharp as that depicted in figure 9. In fact, the interface observed in the EDS map of figure 4 is semi-sharp with gradients of $\mathrm{PbSe}$ and $\mathrm{PbI}_{2}$ appearing close to the interface in the opposite layer. This band structure is important for PbSe conduction, because, as will be discussed shortly, when $\mathrm{PbSe}$ and $\mathrm{PbI}_{2}$ meet the Fermi-level of the $\mathrm{PbSe}$ lowers reducing the resistance to hole flow and increasing the effectiveness of minority carrier traps [12].

We fabricated PbSe detectors to be configured as photoconductors, such that the material conductivity changes upon illumination resulting in a detectable change in current under an applied electric field [10,13]. Even with its long history photoconduction in $\mathrm{PbSe}$ is still a subject of rich investigation [2, 15]. Empirical evidence, described shortly, suggests that the oxygen trap model proposed by Humphrey and Petritz is the dominant model [12].

As described above, PbSe films are grown by chemical bath deposition, and then sensitized by oxidation and halogenation. Humphrey and Petritz analyzed the properties of as-grown, oxidized, and halogenated films [12]. They found their as-grown PbSe films to be n-type, and after oxidation the films were converted to p-type. This is an important finding, because it suggests that the photocurrent generating carriers are holes [10]. Humphrey hypothesized that oxidation resulted in the incorporation of impurities, which acted as electron-traps near the PbSe conduction band. If the trap decay time is greater than the hole-to-contact transit time, then these traps decrease the probability of recombination, and therefore increase the recombination lifetime [16]. In general, equation 1.4 shows that the longer the carrier lifetime the better the performance of the 
photoconductor. Halogenation lowers the Fermi-level thereby increasing the flow of holes in the valence band leading to increased photocurrent [10, 12-13].

\subsubsection{PbSe Carrier Concentration, Mobility, Resistivity, and Film Type}

The Hall Effect, discovered by E. H. Hall in 1879, was used to determine the type, concentration, and mobility of majority carriers within a semiconductor [10, 17]. The basic principles of the Hall Effect starts from the Lorentz force $\mathbf{F}$ in units of newtons $(\mathrm{N})$ shown in equation 2.3.

$$
F=-q(E+v \times B)
$$

Here, $\mathrm{q}$ is the charge constant $\left(1.602 \times 10^{-19}\right.$ coulombs $), \mathbf{E}$ is the electric field in V/cm, $\boldsymbol{v}$ is the velocity, in $\mathrm{cm} / \mathrm{s}$, of the charge as it moves through the electric field $\mathbf{B}$ in units of Gauss. For a Hall measurement, a current-carrying semiconductor is placed within a magnetic field with an orientation orthogonal to the current flow. Charge build up on one side of the semiconductor induces an electric field. In steady state, the force from the electric field and magnetic field balance, resulting in a Lorentz force of $0 \mathrm{~N}$. The built up electric field, known as the Hall field, produces the Hall voltage $V_{H}$, shown in equation 2.4, across the semiconductor in a direction perpendicular to both the direction of current flow and the magnetic field,

$$
\left|V_{H}\right|=E d=\frac{i B}{q n d}
$$

where $\mathrm{E}$ is the magnitude of the electric field in $\mathrm{V} / \mathrm{cm}, \mathrm{d}$ is the thickness of the sample in $\mathrm{cm}, i$ is the magnitude of the current flowing through the semiconductor in amps, B is the magnitude of the magnetic field, and $\mathrm{n}$ is the carrier density in units $\mathrm{of}^{-3}$. The sign of the Hall voltage can be used to determine the majority carrier type in the semiconductor. 
If $\mathrm{V}_{\mathrm{H}}$ is negative then the semiconductor is n-type, and the semiconductor is p-type if it is positive. The $V_{H}$ can be used to find the Hall coefficient $R_{H}$, which is an alternative way of determining the carrier type as described in equation 2.5 .

$$
R_{H}=\frac{-V_{H} d}{B I}
$$

For p-type semiconductors $R_{H}$ will be negative, and $R_{H}$ will be positive for $n$-type [17]. Another important quantity for determining carrier dynamics in semiconductors is the resistivity $\rho$, defined as the product of the sheet resistance in $\Omega /$ square and the sample thickness in $\mathrm{cm}$, which for our films is $1 \times 10^{-4} \mathrm{~cm}$. Shown in equation 2.6 , the resistivity is needed to determine majority carrier mobility $\mu$ with units of $\mathrm{cm}^{2} / \mathrm{Vs}$.

$$
\mu=\frac{R_{H}}{\rho}
$$

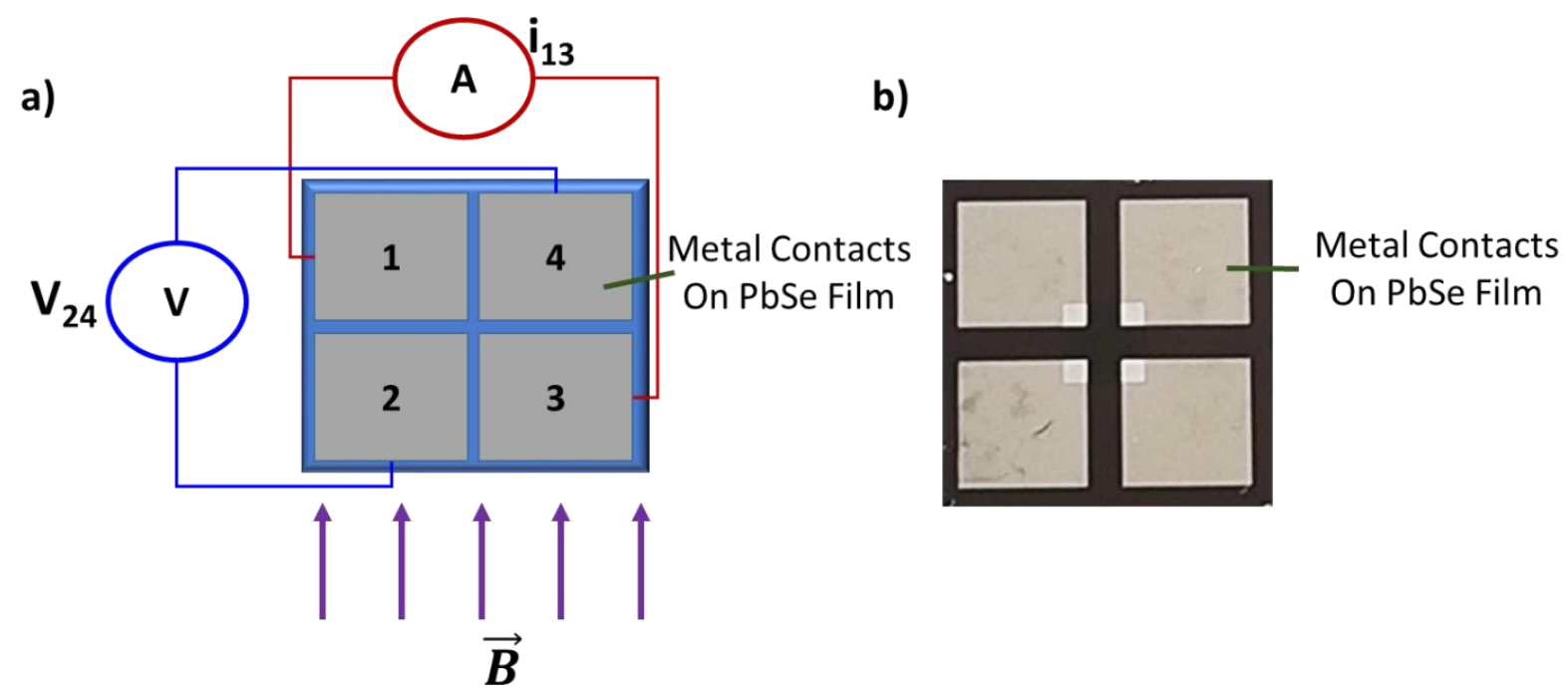

Figure 10. Van der Pauw configuration for measuring Hall parameters; b) PbSe device for Hall measurements where the metal contacts are placed in the four-corners of the PbSe film.

To determine the material properties of the investigated PbSe films, I performed temperature dependent Hall measurements. Metal contacts, in this case made of palladium $(\mathrm{Pd})$, were deposited on the four corners of a $1 \mathrm{~cm} \times 1 \mathrm{~cm}$ sensitized $\mathrm{PbSe}$ 
device with a film thickness of $1 \times 10^{-4} \mathrm{~cm}$. The Hall measurement configuration was that of the Van der Pauw method as depicted in figure 10.

First, the current, denoted as $i_{13}$, is applied across terminals 1 and 3 , while at the same time the voltage, denoted as $V_{24}$, is measured across terminals 2 and 4 . Using Ohm's law, $\mathrm{R}_{\mathrm{A}}$ is the resistance determined with $i_{13}$ and $V_{24}$. Next, $\mathrm{R}_{\mathrm{B}}$ is found in a similar fashion by applying the current across terminals 2 and 4, and measuring the voltage across terminals 1 and 3. Known as the Van der Pauw equation, equation 2.7 shows that $\mathrm{R}_{\mathrm{A}}$ and $\mathrm{R}_{\mathrm{B}}$ can be used to determine the sheet resistance $\mathrm{R}_{\mathrm{S}}$.

$$
1=e^{-\pi R_{A} / R_{S}}+e^{-\pi R_{B} / R_{S}}
$$

To determine the Hall voltage, the sample was placed within a magnetic field of 8000 Gauss, and a source meter was used to apply a current of $500 \mathrm{nA}$ across terminals 1 and 3. The voltage measured across terminals 2 and 4 was the Hall voltage. The configuration was then switched and the current was applied across terminals 2 and 4, while the voltage was measured across terminals 1 and 3 . The two values for the Hall voltage were compared to ensure the measurements were consistent.

Table 2. PbSe film characteristics determined by Hall measurements.

\begin{tabular}{|c|c|c|c|c|c|c|}
\hline Film & $\begin{array}{c}\text { Carrier } \\
\text { Density } \\
\left(\mathbf{c m}^{-3}\right)\end{array}$ & $\begin{array}{c}\text { Carrier } \\
\text { Mobility } \\
\left(\mathbf{c m}^{2} / \mathbf{V s}\right)\end{array}$ & $\begin{array}{c}\text { Sheet } \\
\text { Resistance } \\
(\boldsymbol{\Omega} / \mathbf{s q})\end{array}$ & $\begin{array}{c}\text { Resistivity } \\
(\boldsymbol{\Omega}-\mathbf{c m})\end{array}$ & $\begin{array}{c}\text { Hall } \\
\text { Coefficient } \\
\left(\mathbf{c m}^{3} / \mathbf{C}\right)\end{array}$ & $\begin{array}{c}\text { Film } \\
\text { Type }\end{array}$ \\
\hline As-Grown & $2.76 \times 10^{18}$ & 69.99 & 320 & 0.032 & 2.24 & $\mathrm{n}$ \\
\hline Oxidized & $3.92 \times 10^{17}$ & 44.59 & 3570 & 0.357 & 15.92 & $\mathrm{n}$ \\
\hline $\begin{array}{c}\text { Sensitized } \\
(290 \mathrm{~K})\end{array}$ & $3.03 \times 10^{15}$ & 1.46 & $1.41 \times 10^{7}$ & 1410 & -2060 & $\mathrm{p}$ \\
\hline $\begin{array}{c}\text { Sensitized } \\
(270 \mathrm{~K})\end{array}$ & $5.66 \times 10^{14}$ & 2.85 & $3.86 \times 10^{7}$ & 3860 & -11000 & $\mathrm{p}$ \\
\hline $\begin{array}{c}\text { Sensitized } \\
(230 \mathrm{~K})\end{array}$ & $4.03 \times 10^{14}$ & 3.64 & $4.26 \times 10^{7}$ & 4260 & -15500 & $\mathrm{p}$ \\
\hline
\end{tabular}



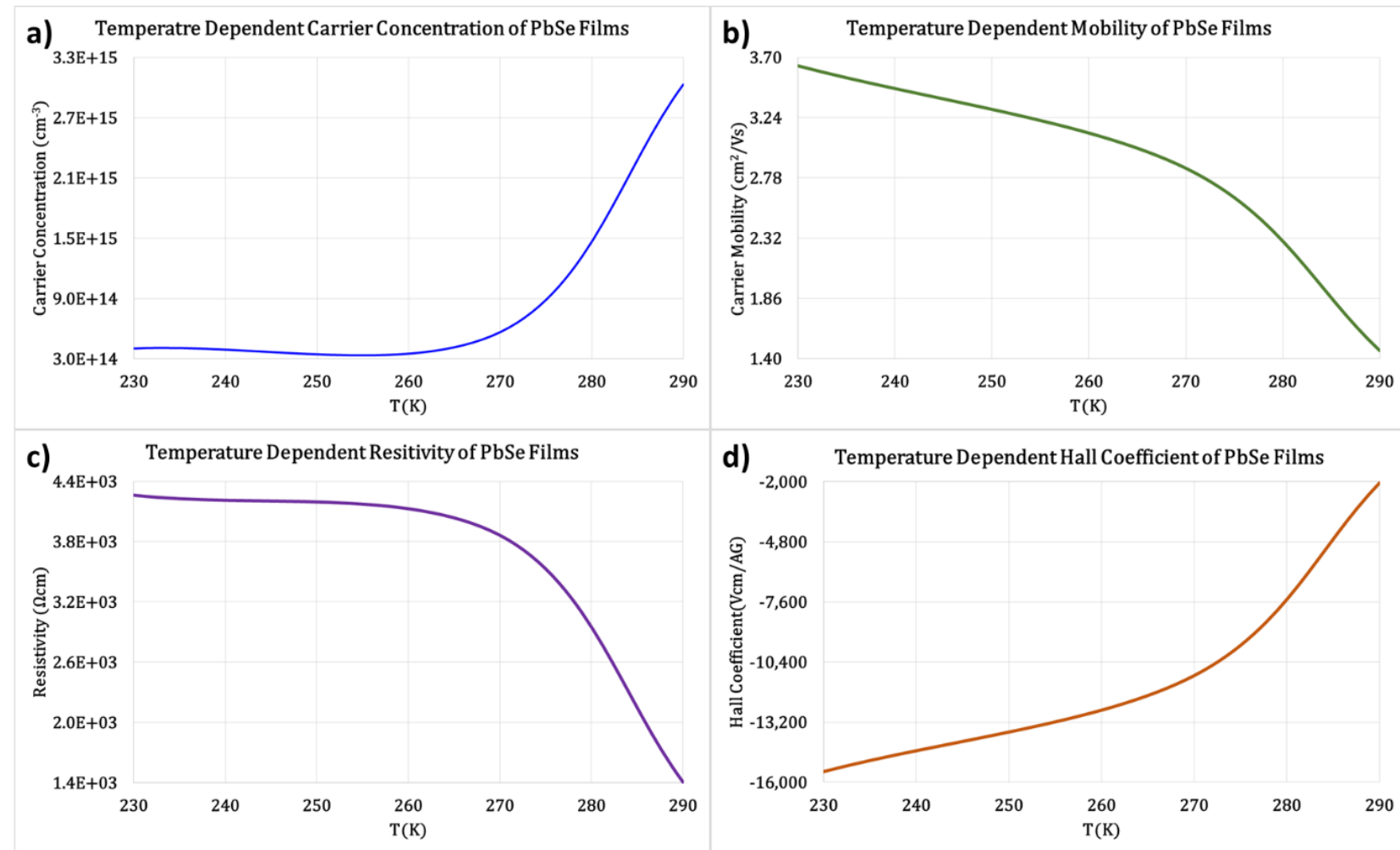

Figure 11. Temperature dependent a) carrier concentration, b) carrier mobility, c) film resistivity, and d) Hall coefficient in sensitized PbSe films. Trends indicate sensitivity increases at lower temperatures.

Table 2 contains room-temperature results we collected and analyzed from asgrown and oxidized $\mathrm{PbSe}$, as well as temperature dependent data of our sensitized devices. These results seem to support the oxygen trap model proposed by Humphrey and Petritz [12]. As-grown films show an n-type carrier concentration on the order of $10^{18}$ $\mathrm{cm}^{-3}$. After oxidation, the free-carrier concentration reduces by approximately an order of magnitude $\left(10^{17} \mathrm{~cm}^{-3}\right)$, suggesting a trapping mechanism. Finally, after sensitization the carrier-concentration reduces by roughly two orders-of-magnitude down to $\sim 10^{15} \mathrm{~cm}^{-3}$, and the polarity switches to p-type. Moreover, the carrier mobility decreases from 70 $\mathrm{cm}^{2} / \mathrm{Vs}$ in as-grown films to $45 \mathrm{~cm}^{2} / \mathrm{Vs}$ in oxidized films, further supporting electron trapping by oxygen impurities. Mobility then decreases to $1.5 \mathrm{~cm}^{2} / \mathrm{Vs}$ in sensitized films. The data presented in table 2 seems to confirm that the incorporation of $\mathrm{PbI}_{2}$ results in 
more effective oxygen traps, and lowers the Fermi-level of PbSe thereby increasing the flow of holes in these p-type films.

The temperature dependent results show expected behavior [10]. As the temperature decreases, the free carrier density, which is dependent on thermal energy $\mathrm{k}_{\mathrm{b}} \mathrm{T}$, reduces. This leads to an increase in mobility, as well as an increase in resistance. Figure 11 shows plots of the carrier concentration, resistivity, and mobility as a function of temperature for sensitized $\mathrm{PbSe}$ films as presented in table 2. The carrier concentration and resistivity change linearly with a steep slope from $290 \mathrm{~K}$ to $275 \mathrm{~K}$, approach an inflection point at $270 \mathrm{~K}$, then level off as the temperature approaches $230 \mathrm{~K}$. Recall from equation 1.4 in Chapter 1 that the sensitivity is inversely proportional to free carrier density. The trend in the carrier concentration and resistivity suggest that there should be similar sensitivity between $260 \mathrm{~K}$ and $230 \mathrm{~K}$. However, the mobility shows a linear increase from $260 \mathrm{~K}$ down to $230 \mathrm{~K}$. Photocurrent is directly proportional to carrier mobility, so as the temperature decreases sensitivity increases [18].

\subsubsection{Minority Carrier Lifetime}

Minority carrier lifetime is an important property for photoconductive detectors. Indeed, its importance is presented in equation 1.4 of Chapter 1 where the higher the minority carrier lifetime the higher the detector sensitivity. To determine this important material property, I measured carrier lifetime of our PbSe films using the photoconductive decay method (PCD) [19], and for comparison my collaborators utilized time resolved photoluminescence (TRPL) [20]. In the PCD method, the circuit depicted in figure 12 is used to measure the change in voltage across a load resistor over time as the PbSe detector is transversely illuminated by pulsed laser light. To ensure the 
measured signal was indicative of the PbSe lifetime, the laser wavelength was selected to be greater than the absorption cutoff of $\mathrm{PbI}_{2}$ [11]. The carrier lifetime is defined as the time for the excess carrier concentration $\mathrm{n}$ in $\mathrm{cm}^{-3}$, generated from the formation of electron-hole pairs during excitation, to decay to a value of approximately $36.7 \%$ (1/e) of its initial value $\mathrm{n}_{0}$

$$
n(t)=n_{0} \exp \left(-\frac{t}{\tau}\right)
$$

where $t$ is the time in seconds within a single period over which the measurement is taken. In the photoconductive decay method, $\mathrm{n}$ is proportional to the measured voltage $\mathrm{V}$, such that

$$
V(t)=V_{0} \exp \left(-\frac{t}{\tau}\right)
$$

The value of the carrier lifetime $\tau$, with units typically given as $\mu$ s or ns, can be found by fitting an exponential decay curve, shown in equation 2.10 , to the measured decay of the voltage.

$$
y(t)=y_{0} \exp (-A x)
$$

By equating equations 2.9 and 2.10 it is evident that $y_{0}$ is $\mathrm{V}_{0}$ and $\mathrm{Ax}$ is equal to $t / \tau$ from a similarity argument. Taking the natural log of both sides, and rearranging equation 2.10 results in the value of the lifetime shown in equation 2.11 .

$$
\tau=\frac{t}{\ln \left(\frac{V_{0}}{V(t)}\right)}
$$

Lead selenide test detectors were fabricated as described in Appendix A, with a 7 $\mathrm{mm} \times 7 \mathrm{~mm}$ active area, and metal contacts on either side of sensitized film. Figure 13 shows the observed photoconductive decay curve. The test detector was biased using a 9 
V battery, while the active area was illuminated by a model B071 pulsed laser from Power Technology, Inc. with a wavelength of 980 nm, pulse repetition frequency of 100 $\mathrm{Hz}$, pulse width of $20 \mathrm{~ns}$, and a measured average power of $1.3 \mathrm{~mW}$ [47]. The decay spectra was normalized to 1 , and an exponential fit was used to determine the lifetime as described above.

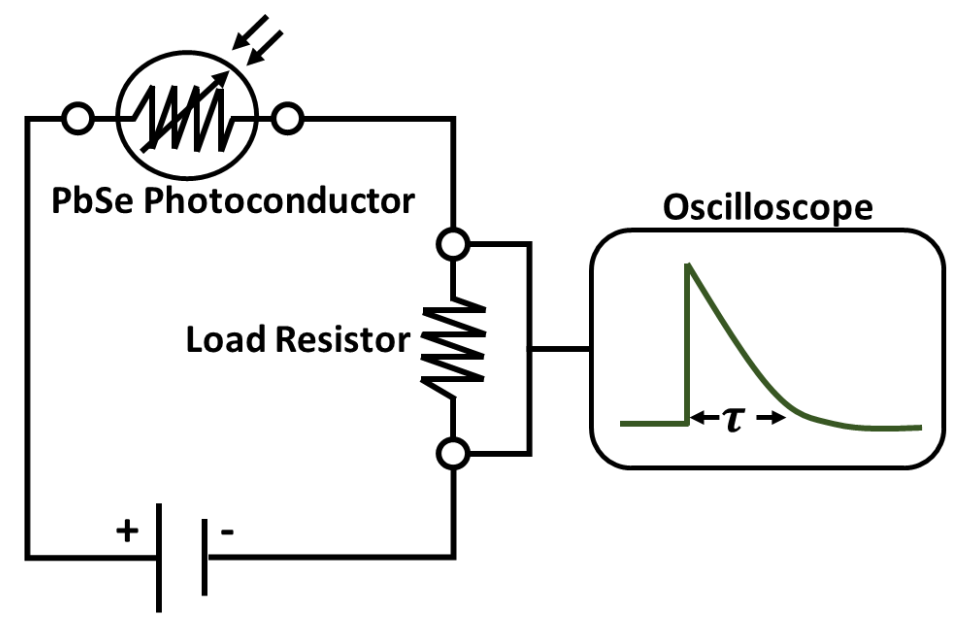

Figure 12. Bias circuit for measuring minority carrier lifetime in PbSe films.

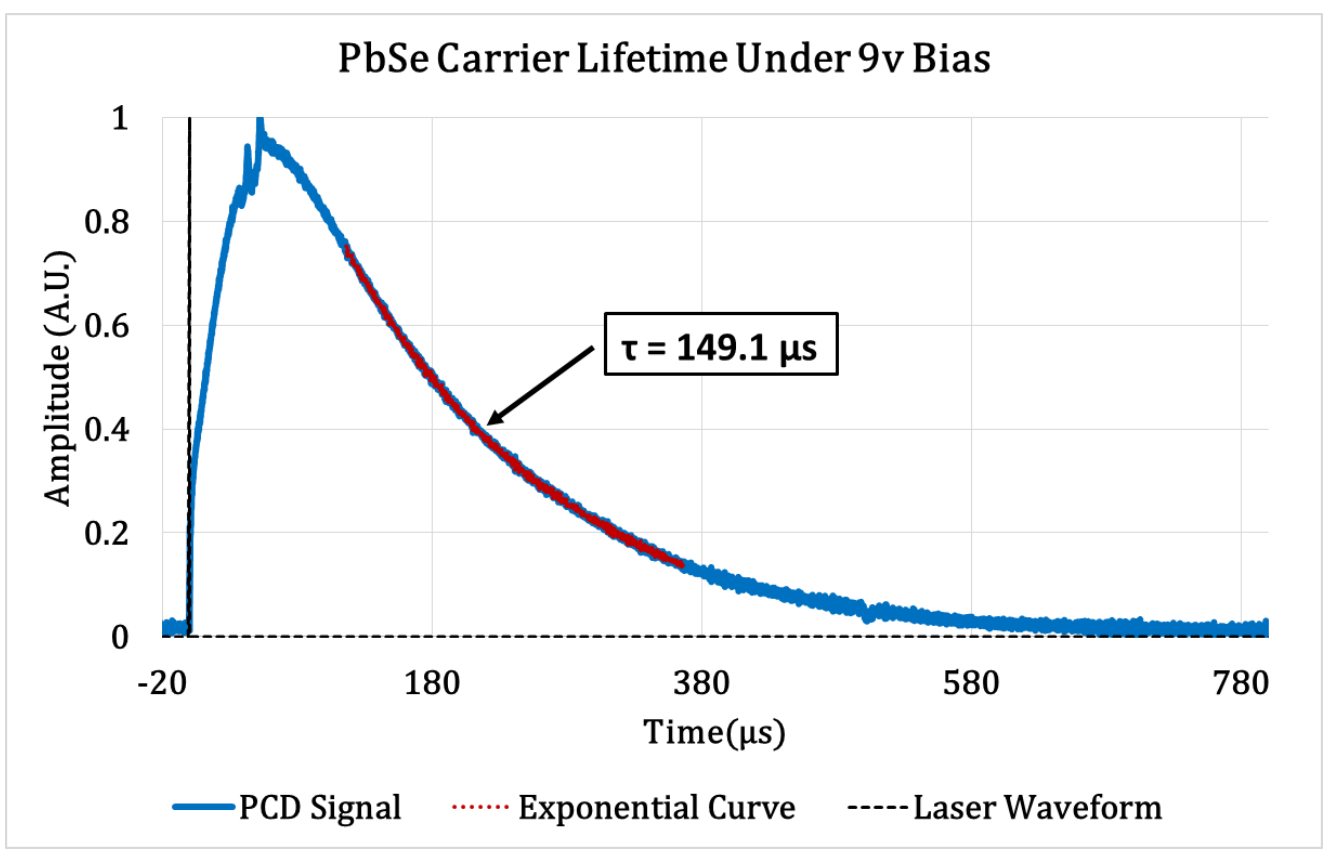

Figure 13. The room temperature PCD curve observed by transversely illuminating a PbSe test detector with pulsed laser light, and measuring the change in voltage across a load resistor connected in series. The carrier lifetime was extracted by fitting an exponential curve (red trace) to the decay data (blue trace). 
For sensitized PbSe detectors, the carrier lifetime as measured by the PCD method was found to be $\sim 149 \mu \mathrm{s}$. We have previously reported that the carrier lifetime of these detectors varies from $30 \mu$ s to greater than $150 \mu$ s at room temperature depending on deposition conditions and metal contacts [7]. This is quite large when compared to previously reported carrier lifetimes of $2-10 \mu$ s in other PbSe films $[14,12]$.

I verified carrier lifetime by also measuring the RC-time constant, and ensuring it was longer than the measured PCD decay time. In a configuration similar to that of the PCD method, the RC time constant was found by measuring the decay time across the load resistor as the bias circuit was pulsed using an arbitrary waveform generator (AWG) [21]. In this configuration, the sample is kept dark such that the observed signal is only due to the electrical pulse from the AWG. Figure 14 shows the configuration of an RCtime constant measurement, and figure 15 shows the RC-time constant data for the same detector under similar test conditions as figure 13.

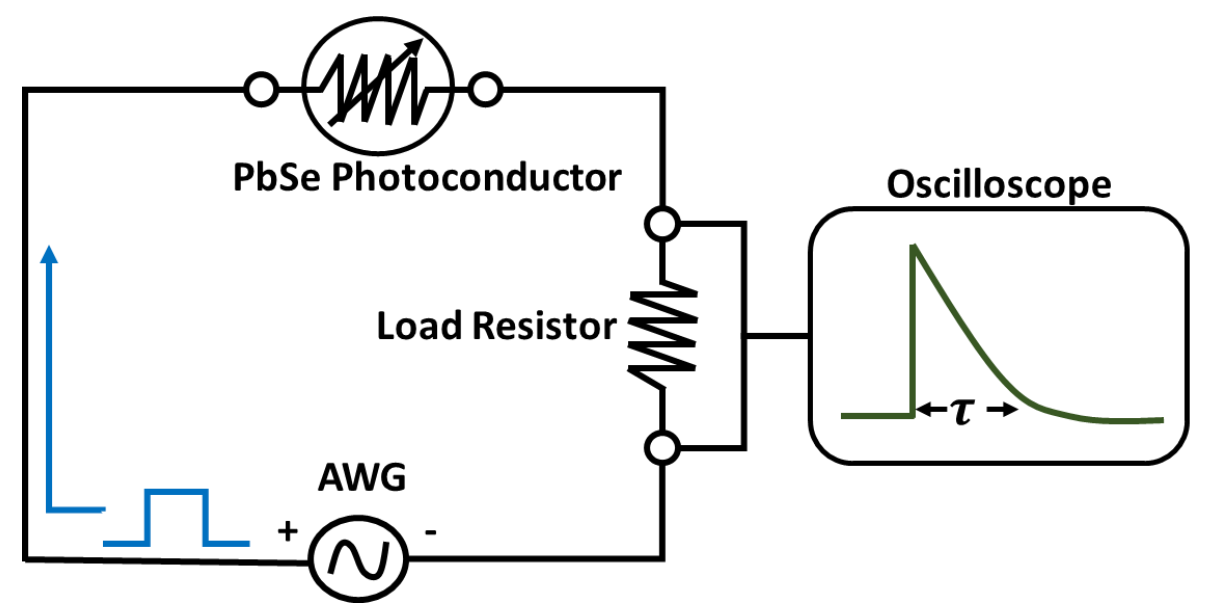

Figure 14. An RC-time constant measurement setup. 


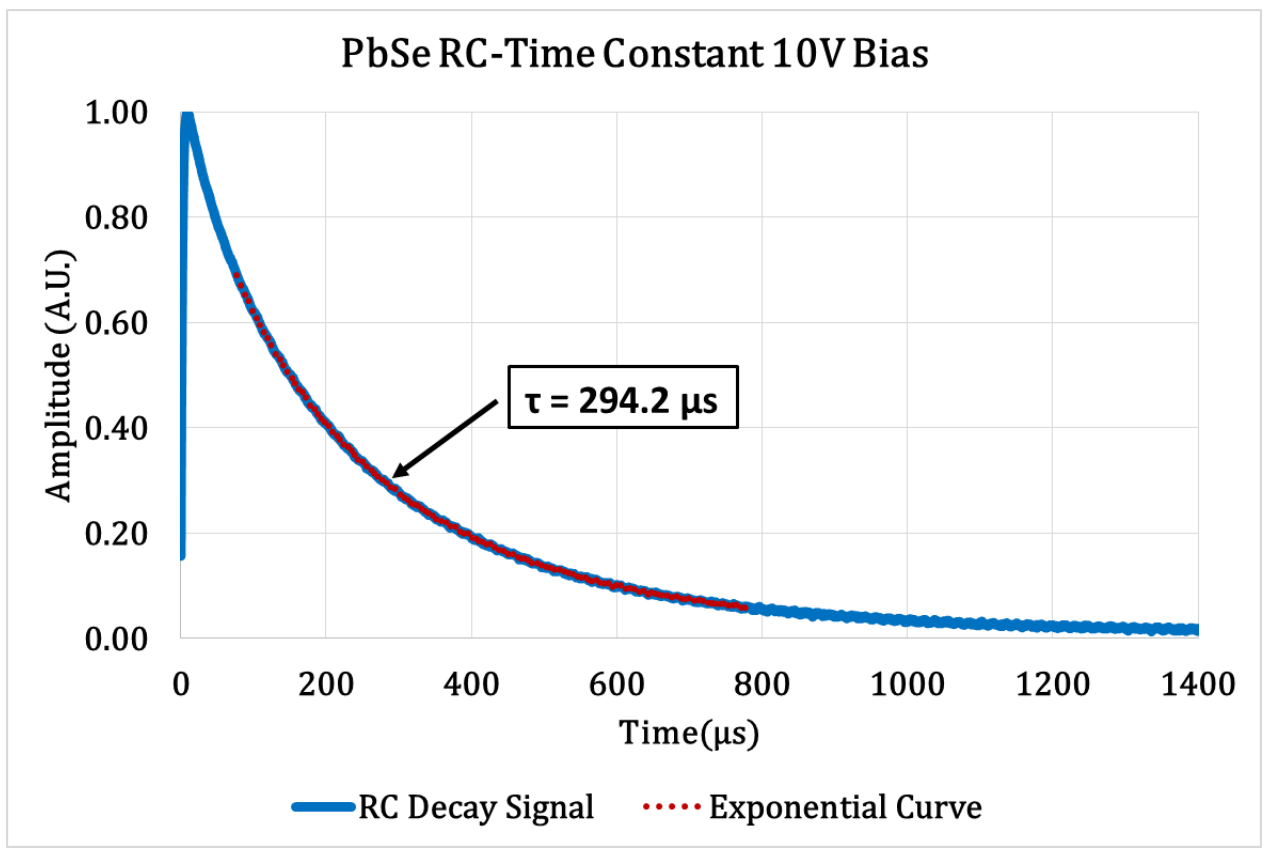

Figure 15. The RC-decay curve measured across the PbSe detector as it was pulsed by an AWG. The decay curve shows a factor of two increase over that of the PCD measurement, distinguishing the measured lifetime from the RC time constant.

The RC-time constant was calculated to be $294 \mu$ s using a $100 \mathrm{~Hz}$ pulse with an amplitude of $10 \mathrm{~V}$ to electrically excite the detector without illumination. The PCD carrier lifetime for the same detector biased from $9-25 \mathrm{~V}$ was found to be $149-168 \mu \mathrm{s}$. The drift velocity of holes is in the direction of the applied electric field [10,13]. Therefore, assuming oxygen trapping is the dominant mechanism for photoconduction, when the bias is increased across the $\mathrm{PbSe}$ detector, the drift current increases further reducing the probability of recombination. Notice that the measured RC-time constant is only two-times greater than the measured carrier lifetime. As a final verification for the measured lifetime, the carrier lifetime found by the PCD method was subtracted from the RC-time constant yielding a difference of $145.1 \mu \mathrm{s}$. This difference is within $2.7 \%$ of the calculated carrier lifetime, which shows the measured PCD curve and RC-decay curve were indeed distinguished from one another, confirming the validity of the results. 
Depicted in figure 16, our collaborators measured time resolved photoluminescence (TRPL) on the same PbSe devices [20]. PbSe test detectors were placed at the focus of a 100x microscope objective. A pulse signal, with pulse repetition frequency of $100 \mathrm{kHz}$, was sent from an arbitrary waveform generator (AWG) to trigger both the laser, and the start of the measurement time. The beam from a $1.064 \mu \mathrm{m}$ laser with a pulse width of $20 \mathrm{~ns}$ was reflected off of a long-wavelength pass dichroic mirror into the entrance aperture of the microscope objective. The beam was focused onto the sample to excite carriers and produce photoluminescence. The photoluminescence signal, which consisted of longer wavelength light than that of the pump beam, was collected by the microscope objective and projected through the dichroic mirror onto the detector head. Similar to the PCD method, the signal decay was monitored over time and analyzed to determine the carrier lifetime. In contrast to the PCD method, the PbSe detector was not biased during this measurement.

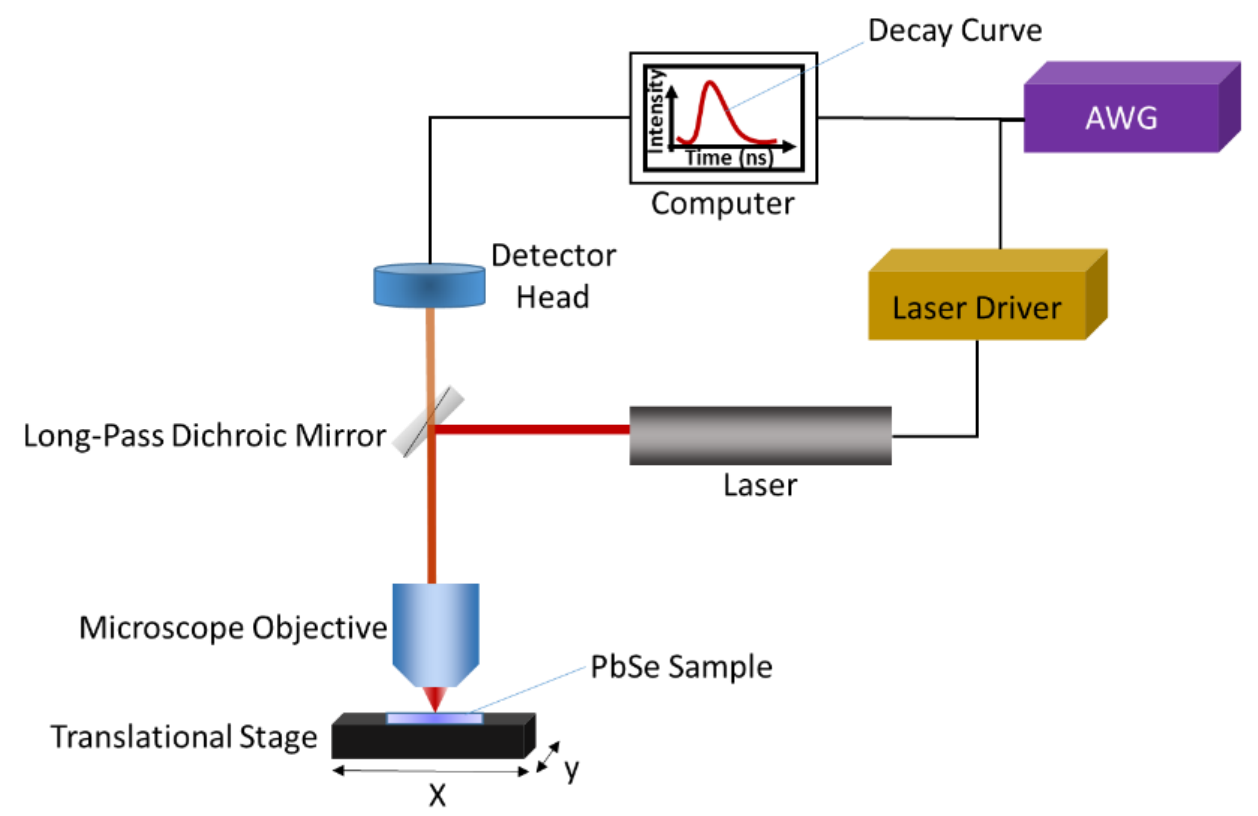

Figure 16. Time resolved photoluminescence setup for carrier lifetime measurements on PbSe test detectors. 
The observed decay curve from TRPL measurements is shown in figure 17. The decay of the trace is not smooth like in figures 13 and 15 . Therefore, the lifetime was estimated by simply finding the 1/e point after the peak of the intensity was reached. The lifetime determined by TRPL was found to be $92 \mathrm{ns,} \mathrm{which} \mathrm{is} \mathrm{three} \mathrm{orders-of-magnitude}$ less than the PCD results suggested. Ahrenkiel observed the same disparity when comparing PCD and TRPL results obtained from measuring polycrystalline $\mathrm{HgCdTe}$ thin films [22]. The difference between the results of the two techniques can be understood, again, by carrier trapping. The TRPL measurement requires radiative recombination, and is therefore not sensitive to carrier traps. The density of states of the trap level is too low for an appreciable photoluminescence signal at room temperature [22]. However, PCD is very sensitive to carrier trapping, because it measures the decay of current carriers through the semiconductor material. Furthermore, depending on the type of metal contacts, the barrier height, and therefore the carrier lifetime, will change [7].

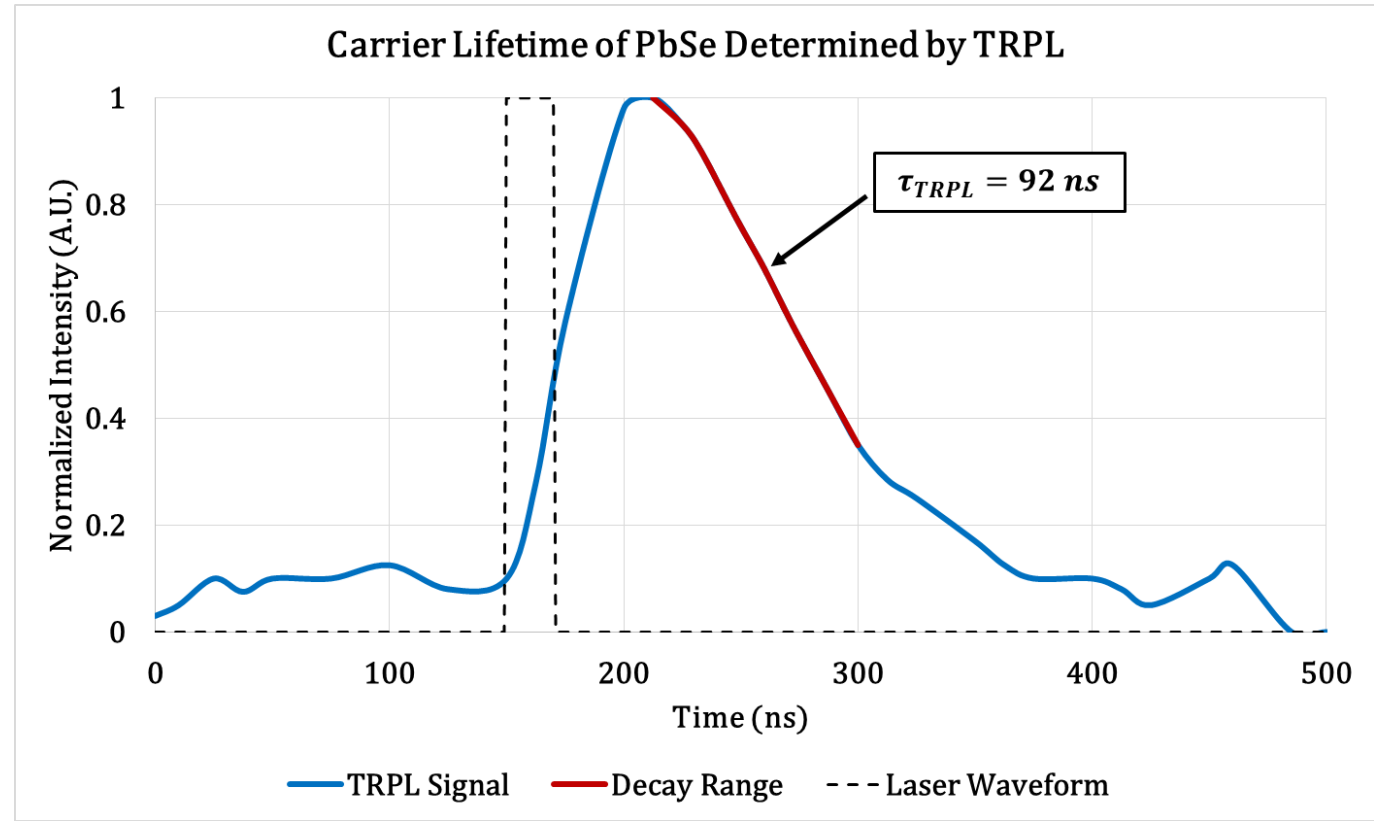

Figure 17. Observed TRPL decay curve for PbSe test detector at room temperature. Results show a threeorder-of-magnitude disparity compared to those of the PCD method due to carrier trapping. 
Another photoconduction model has been proposed by Kuman and Lihuazhao $[15,23]$. Their charge separation model suggests that the core of the PbSe polycrystalline grains is n-type, while the surface is p-type. The p-type carriers flow in one direction in the spacing between grains, while the n-type carriers flow between the grains in the opposite direction thereby separating the charges leading to long lived carrier lifetimes and greater performance. There are, however, differences between the $\mathrm{PbSe}$ films on which [14] and [23] report and the films we have investigated. The largest difference between the films of this investigation and those reported in literature is the fact that the films in the literature start as p-type, and then become n-type in as-grown and sensitized samples. Furthermore, the charge separation model assumes iodine acts as a dopant to increase the number of carriers in the film. Though the charge separation model is feasible, the experimental evidence presented above seems to suggest that the films we investigated adhere more to the oxygen trap model of Humphrey and Petritz [12].

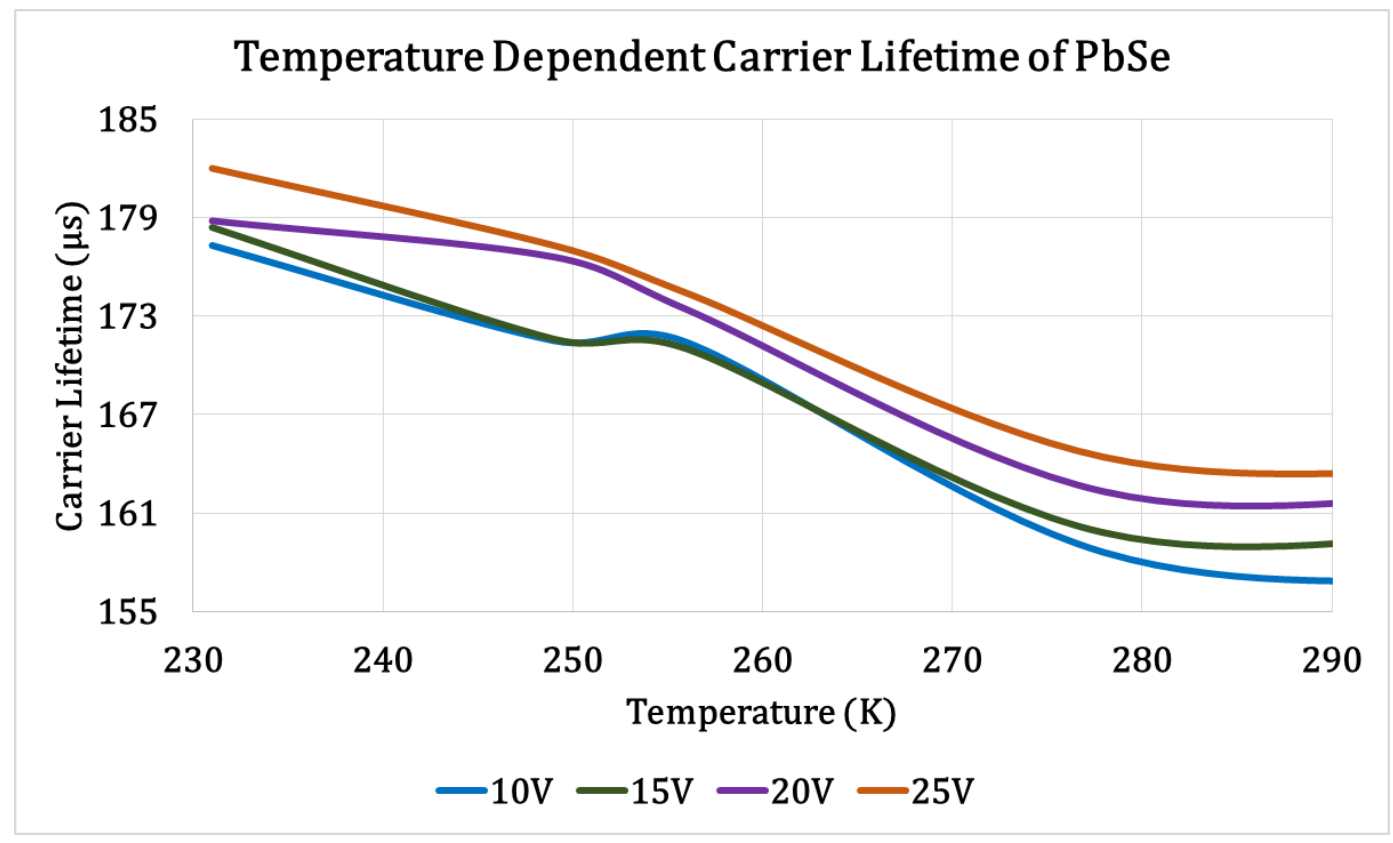

Figure 18. Observed temperature and bias dependence of the carrier lifetime of PbSe test detectors measured using the PCD method. 
In addition to room temperature measurements, I also measured the temperature dependence of the carrier lifetime. Test detectors were mounted in a thermoelectric cooler (TEC) dewar assembly, and the carrier lifetime was measured at $290 \mathrm{~K}, 270 \mathrm{~K}$, $250 \mathrm{~K}$, and $230 \mathrm{~K}$ at bias levels between $10 \mathrm{~V}$ and $25 \mathrm{~V}$. The data in figure 18 shows that for all biases, the carrier lifetime increases with decreasing temperature. This result is expected, because the excess carrier concentration should decrease as the temperature decreases due to its energy $\left(\mathrm{k}_{\mathrm{b}} \mathrm{T}\right)$ dependence $[10,13]$. Of note is the fact that the total increase in carrier lifetime from $290 \mathrm{~K}$ to $230 \mathrm{~K}$ was only found to be $30 \mu \mathrm{s}$. This small increase suggests that the performance of these $\mathrm{PbSe}$ films at room temperature is similar to that of $230 \mathrm{~K}$, and only a small enhancement is needed for comparable performance at room temperature.

\subsection{Absorption in PbSe}

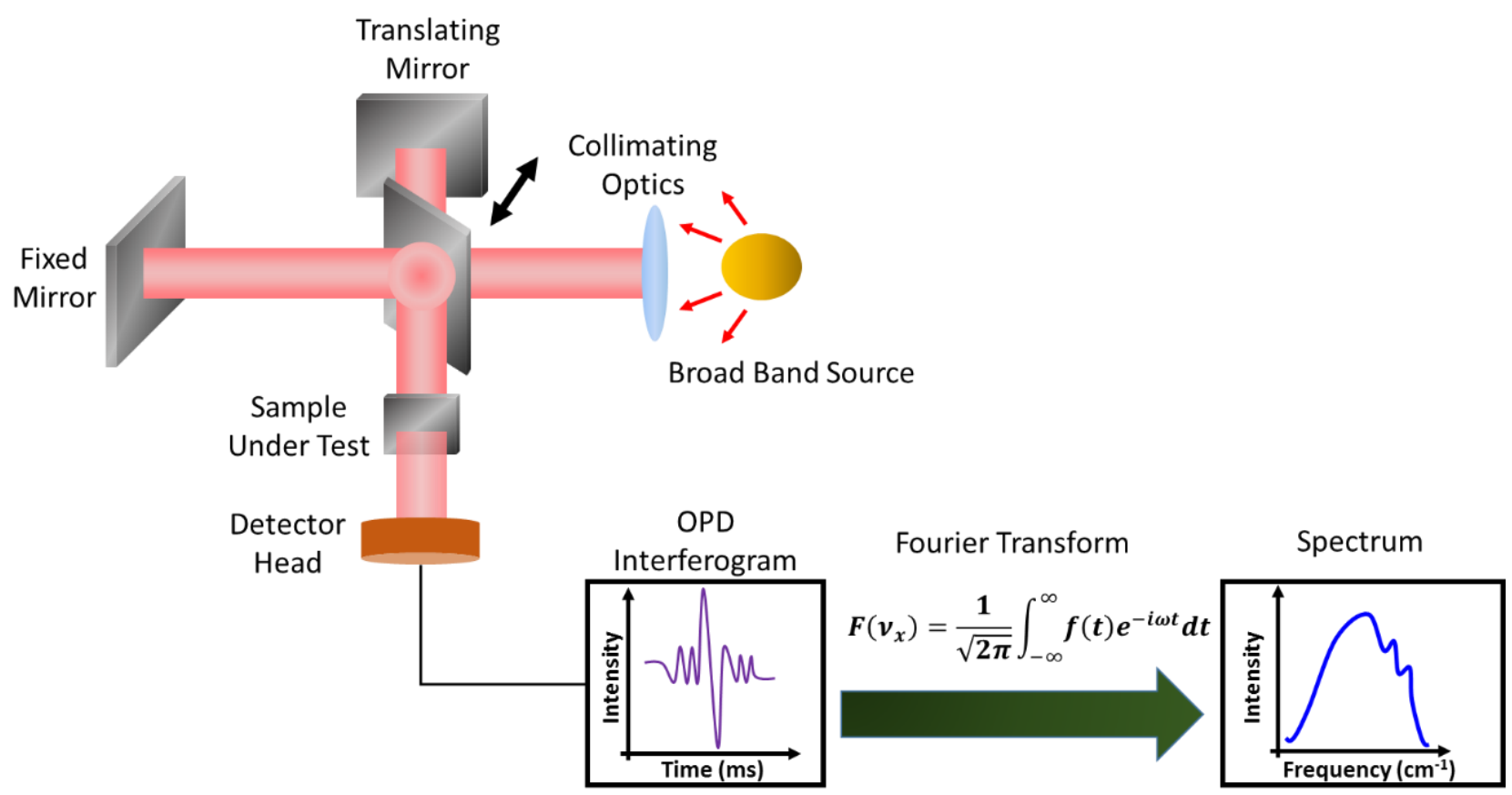

Figure 19. Depiction of FTIR measurement. 
Recall that the baseline films within this study have a peak-to-peak surface roughness of $500 \mathrm{~nm}$. This roughness makes it difficult to determine the absorption using methods such as Fourier transform infrared spectroscopy (FTIR). Depicted in figure 19, during an FTIR measurement, light from a blackbody emitter is transmitted through a Michelson interferometer where one leg of the interferometer is translated back and forth to modulate the signal in time creating an optical path difference between the beams of the two legs [24-25]. When using a broadband blackbody source, the resulting interferogram will contain peaks corresponding to constructive interference for the regions of zero-path difference. This interferogram is analyzed via a Fourier transform shown in equation 2.12 , which relates the time spectrum $f(t)$ to the spatial frequency spectrum $F\left(v_{x}\right)$, to determine the spectral content, as well as intensity, of the resulting spatial frequency spectrum in units of $\mathrm{cm}^{-1}$. The transmittance, reflectance, and absorbance of a sample can be determined by placing it in the optical path before the detector.

$$
F\left(v_{x}\right)=\frac{1}{\sqrt{2 \pi}} \int_{-\infty}^{\infty} f(t) e^{-i \omega t} d t
$$

Transmission spectra are relatively simple to determine, yet scattering by diffuse and rough surfaces can lead to indistinguishable spectral features [25]. Moreover, when the absorption or reflection coefficients are desired, without a priori knowledge of either the absorbance or reflectance, the quantity that can be determined with certainty is the optical density OD which includes contributions from reflection, including scattering, and absorption. The optical density is given by equation 2.13 ,

$$
O D=-\log (T)
$$


where $\mathrm{T}$ is the measured transmission with a maximum value of 1 . One way to circumvent this limitation and determine the absorption within thin films is to insert the sample in the middle of an integrating sphere, and sense the transmitted light output by the sphere [26]. The inside of an integrating sphere is made of a diffuse Lambertian scattering surface, which is a surface that scatters in all directions evenly resulting in a uniform output. When a sample is put within an integrating sphere, the incident light will propagate through the input port and undergo Fresnel reflections at the interfaces within the film. All of the incident light will be scattered and reflected out of the integrating sphere onto an optical detector, so the only attenuation will be from absorption within the sample material. Using this configuration, the light from an FTIR auxiliary port was focused onto the input port of an integrating sphere with a sensitized PbSe sample suspended from one of the sample ports. The output of the integrating sphere was positioned in front of a $\mathrm{HgCdTe}$ MWIR detector cooled to $77 \mathrm{~K}$. The signal of the detector was then input to the external detector port of the FTIR for analysis.

To measure the absorption in these films, I inserted sensitized PbSe film samples into a gold integrating sphere with a spectral reflectance from $1 \mu \mathrm{m}-20 \mu \mathrm{m}$ of greater than $95 \%$. To ensure minimal absorption by outside sources, the sample was suspended from one of the sphere sample ports by a gold wire. Using equation 2.14 , the absorption in the film was determined.

$$
A=1-10^{-O D}=1-10^{\log (T)}
$$

Figure 20 shows the absorption spectra obtained from one of our sensitized $\mathrm{PbSe}$ film samples. The maximum absorption above the cutoff wavelength at $4.3 \mu \mathrm{m}$ is $35 \%$ across the 3-5 $\mu \mathrm{m}$ spectral band. The decrease in absorption around $3.2 \mu \mathrm{m}$ and $4.3 \mu \mathrm{m}$ is 
associated with $\mathrm{H}_{2} \mathrm{O}$ and $\mathrm{CO}_{2}$ in the atmosphere [27-28]. Further, the increase in absorption between $4.8 \mu \mathrm{m}$ and $6.0 \mu \mathrm{m}$ is associated with carbon impurities in the underlying $\mathrm{SiO}_{2}$ [29]. Assuming a maximum absorption of $100 \%$, these results suggest the theoretical maximum in-band absorption enhancement below the cutoff wavelength is a factor of approximately 2.85. Moreover, these results also suggest the maximum external quantum efficiency in baseline $\mathrm{PbSe}$ detectors without resonant structures at room temperature is $35 \%$. However, as will be discussed shortly, the observed quantum efficiency in baseline $\mathrm{PbSe}$ detectors was much lower due to both radiative and nonradiative carrier recombination [10].

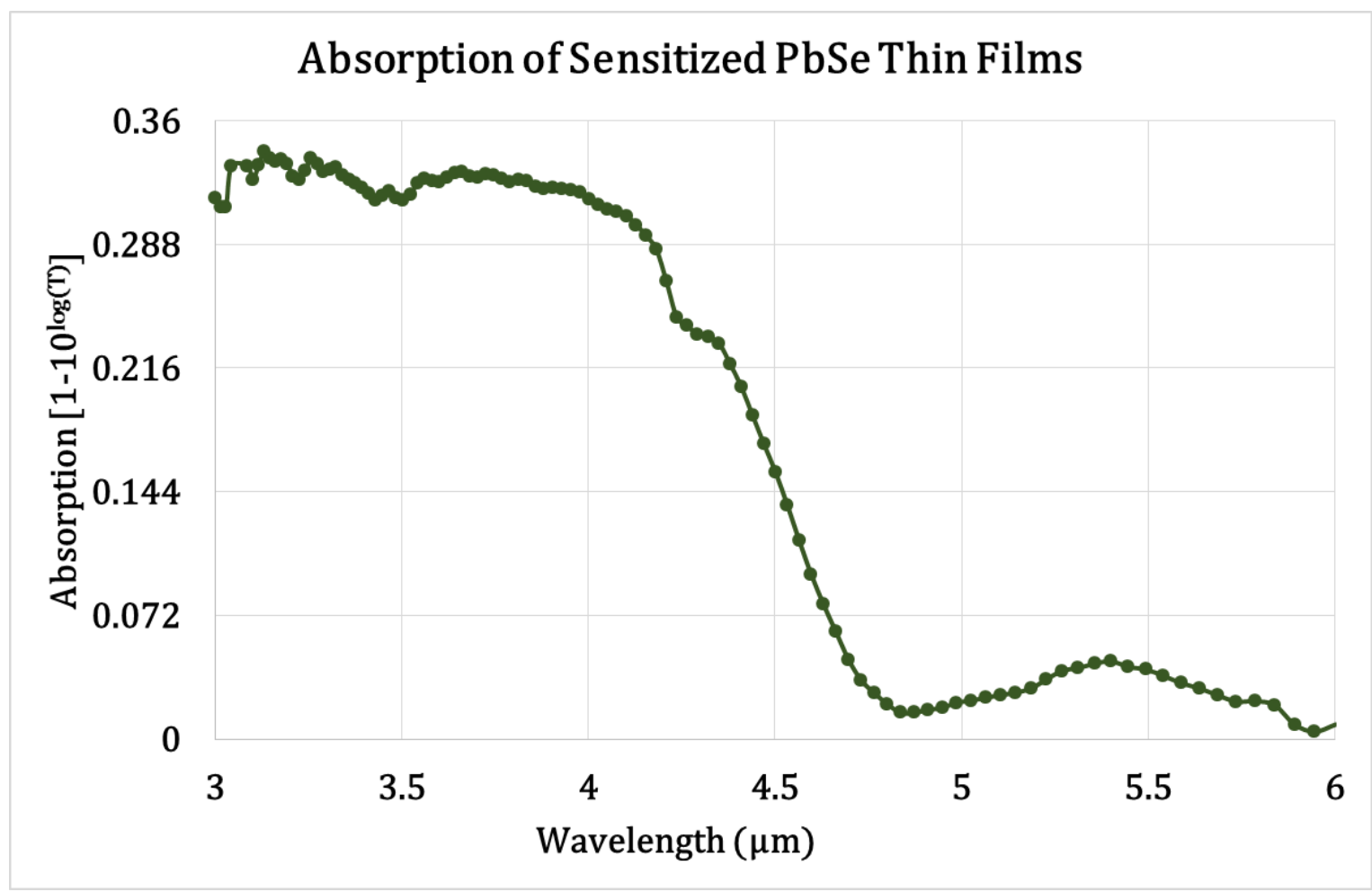

Figure 20. Absorption spectrum of investigated PbSe thin films at $290 \mathrm{~K}$ determined by FTIR with integrating sphere. 


\subsection{Performance of Baseline PbSe Test Detectors}

An important parameter for understanding the performance of infrared photoconductors is the wavelength dependent photoresponse. I measured the spectral response of $\mathrm{PbSe}$ test detectors using the same bias circuit configuration as that depicted in figure 12. The output from the bias circuit was input into an AMETEK Scientific Instruments model 5210 lock-in amplifier, the output of which was then used as the auxiliary detector input for a Nexus model 870 Fourier transform infrared spectrometer. The responsivity $\mathfrak{R}$ was measured over a spectrum of $10000 \mathrm{~cm}^{-1}$ to $1800 \mathrm{~cm}^{-1}(1 \mu \mathrm{m}-$ $5.5 \mu \mathrm{m})$. I calculated the FTIR source power spectral density by measuring the spectral response of a Thor Labs model FDPSE2X2 $2 \mathrm{~mm}$ x $2 \mathrm{~mm}$ PbSe photoconductor, interpolating the response curve derived from information in the detector manual, and taking the quotient between the measured and interpolated curves [30]. The calculated source power can be seen in figure 21.

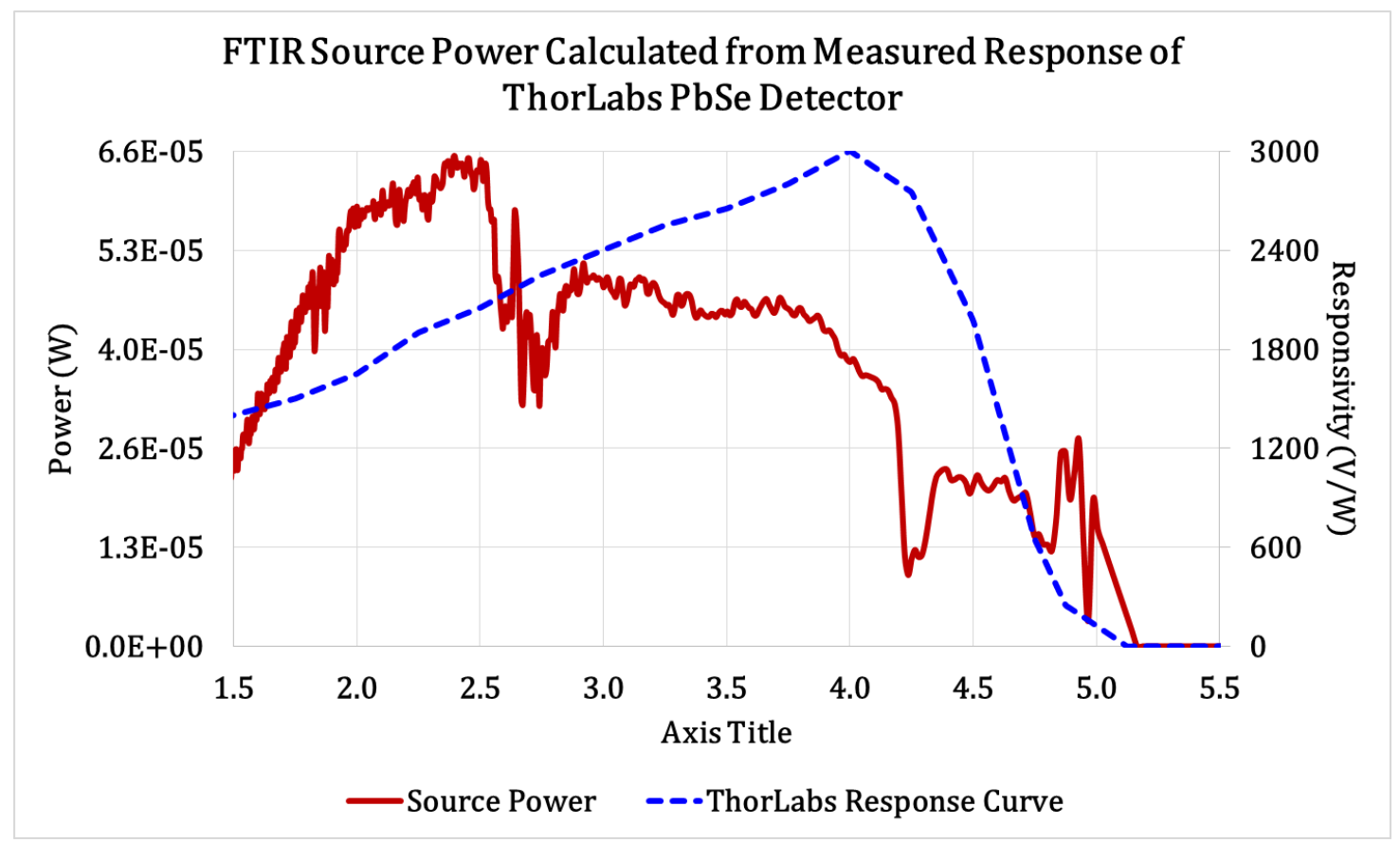

Figure 21. Reported Thor Labs responsivity (blue, dashed), and calculated FTIR source power spectrum (red, solid). 


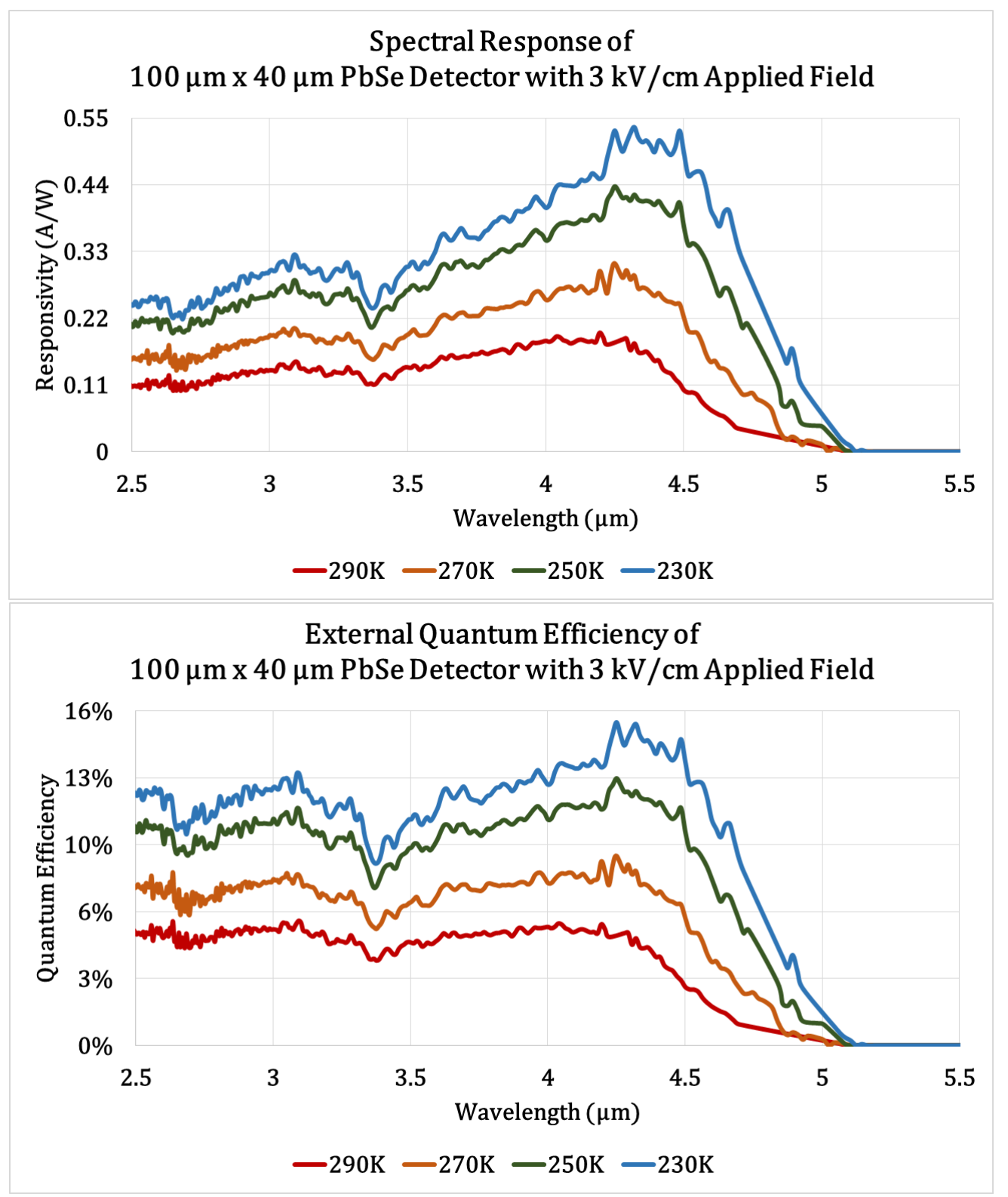

Figure 22. Measured responsivity and external quantum efficiency of a $100 \mu \mathrm{m} x 40 \mu \mathrm{m}$ PbSe test detector under an applied electric field of $3.3 \mathrm{kV} / \mathrm{cm}$ at ambient temperatures of interest.

The change in voltage across the $11 \mathrm{M} \Omega$ load resistor $\mathrm{R}_{\text {load }}$ was measured as the $\mathrm{PbSe}$ test detector was illuminated, and $\mathfrak{R}$, with units of $\mathrm{A} / \mathrm{W}$, was found using equation 2.15 ,

$$
\Re(\lambda)=\frac{\mathrm{V}_{\mathrm{m}}(\lambda) \mathrm{A}_{\mathrm{TLPbSe}}}{\mathrm{P}_{\mathrm{S}}(\lambda) \mathrm{R}_{\text {load }} \mathrm{A}_{\mathrm{PbSeTD}}}
$$


where $\mathrm{V}_{\mathrm{m}}$ is the measured voltage difference upon illumination across the load resistor $\mathrm{R}_{\text {load }}$ in ohms, $\mathrm{A}_{\text {TLPbSe }}$ is the area of the reference detector in $\mathrm{cm}^{2}, \mathrm{P}_{\mathrm{s}}$ is the calculated source power in watts, $\lambda$ is the wavelength in $\mu \mathrm{m}$, and APbSeTD is the area of the PbSe detector under test in $\mathrm{cm}^{2}$. From the responsivity, the external quantum efficiency, defined as the ratio of the number of carriers that contribute to photocurrent to the number of incident photons, can be found by using equation 2.16 .

$$
\eta_{e}=\frac{\Re h v}{q}
$$

Figure 22 shows the spectral response and quantum efficiency for a $100 \mu \mathrm{m}$ x 40 $\mu \mathrm{m}$ detector under an applied field of $3 \mathrm{kV} / \mathrm{cm}$ at temperatures ranging from $290 \mathrm{~K}$ down to $230 \mathrm{~K}$. The peak responsivity and external quantum efficiency at $4.3 \mu \mathrm{m}$ and $230 \mathrm{~K}$ were found to be $0.55 \mathrm{~A} / \mathrm{W}$ and $15 \%$, respectively. Notice the decrease in response and quantum efficiency at $\sim 3.4 \mu \mathrm{m}$. To be discussed in detail in Chapter 4 , this drop in sensitivity is due to optical interference. These are the baseline values from which the sensitivity enhancement gained by incorporating optically resonant structures into $\mathrm{PbSe}$ photoconductors were measured.

\subsection{Dispersion relations of $\mathrm{PbSe}$}

The dispersion relations, which define how the complex index of refraction of a material changes with the illumination wavelength, determine the behavior of electromagnetic waves as they pass through and interact with the material [31-32]. Reflection and transmission are most often associated with the real part of the complex index, whereas absorption is associated with the imaginary part. The complex index of refraction $\mathrm{m}$ is given by equation 2.17 , 


$$
m=n+i \kappa
$$

where $\mathrm{n}$ is the real part of the index, $\kappa$ is the imaginary part, and $i$ denotes the imaginary number $\sqrt{-1}$. Typically, ellipsometry is used to determine the optical constants of the thin film materials [33].

Table 3. Bauer and Krenn's PbSe dispersion relations at an ambient temperature of $290 \mathrm{~K}$.

\begin{tabular}{|c|c|c|}
\hline $\begin{array}{c}\text { Wavelength } \\
(\mu \mathrm{m})\end{array}$ & n & k \\
\hline 2 & 4.7 & 0.25 \\
\hline 2.066 & 4.7 & 0.254 \\
\hline 2.254 & 4.7 & 0.238 \\
\hline 2.48 & 4.7 & 0.45 \\
\hline 2.583 & 4.9 & 0.4 \\
\hline 2.695 & 4.88 & 0.25 \\
\hline 2.755 & 4.883 & 0.22 \\
\hline 2.818 & 4.885 & 0.22 \\
\hline 2.952 & 4.895 & 0.22 \\
\hline 3.024 & 4.9 & 0.22 \\
\hline 3.1 & 4.91 & 0.22 \\
\hline 3.179 & 4.85 & 0.219 \\
\hline 3.263 & 4.93 & 0.225 \\
\hline 3.444 & 4.95 & 0.22 \\
\hline 3542 & 4.95 & 0.199 \\
\hline 3647 & 4.96 & 0.194 \\
\hline 3757 & 4.97 & 0.189 \\
\hline 3875 & 4.98 & 0.173 \\
\hline 4000 & 4.97 & 0.127 \\
\hline 4275 & 4.95 & 0.0572 \\
\hline 4428 & 4.94 & 0.0353 \\
\hline 4592 & 4.94 & 0.00917 \\
\hline 4769 & 4.94 & 0.00285 \\
\hline 4959 & 4.92 & 0.00154 \\
\hline 5166 & 4.9 & 0.00138 \\
\hline 5391 & 4.83 & 0.00128 \\
\hline 5636 & 4.86 & 0.0012 \\
\hline
\end{tabular}


However this technique requires the angle of reflected light to be determinate, and random scattering from the roughness in these films makes this measurement difficult. Therefore, for PbSe, the room temperature complex index of refraction, shown in table 3, was taken from Bauer and Krenn [34].

It has been reported that the $\mathrm{PbSe}$ cutoff wavelength undergoes a redshift as the ambient temperature decreases $[1,7,37]$. This redshift is due to a decrease in the bandgap energy at lower temperature, which is unique to PbSe and other lead-salt materials [3637]. To understand and estimate how the complex index of refraction of $\mathrm{PbSe}$ changes with temperature, I quantified the red-shift in cutoff wavelength utilizing photoluminescence (PL) spectroscopy to observe the change in bandgap with temperature.

In photoluminescence spectroscopy, a semiconductor material is illuminated by either CW or pulsed laser light. If the energy of the incident beam is greater than that of the bandgap, an electron in the valence band can absorb an incoming photon and be excited into the conduction band of a direct bandgap semiconductor $[10,13,17]$. Unless the energy of excitation is equal to that of the bandgap, the electron will be excited to an energy state above the bottom of the conduction band leaving a positively charged core or hole at the top of the valence band. The time for the electron to relax to a lower energy level, known as the metastable state, will be on the order of picoseconds [38-39]. This transition process is non-radiative, and will not result in a photon. The electron will remain in the metastable state, which in semiconductors corresponds to the bottom of the conduction band, for time periods on the order of 10s of microseconds where it becomes part of a bound electron-hole pair known as an exciton [13]. If the semiconductor 
material under test is a direct bandgap material, defined as a material in which the bottom of the conduction band and the top of the valence band are aligned in momentum space, the electron will decay, recombine with its corresponding hole, and undergo the process of annihilation resulting in an emitted photon with an energy that matches the energy difference between the metastable state and the valence band [17, 39]. The photoluminescence process is depicted in figure 23, and a depiction of the PL setup is shown in figure 24 .

Sensitized PbSe test films were mounted in a closed cycle dewar-cooler assembly, and illuminated by a $1.064 \mu \mathrm{m}$ neodymium-doped yttrium aluminum garnet (Nd:YAG) laser at an incident angle of $45^{\circ}$. The resulting PL signal was emitted in the direction perpendicular to the sample face, and was detected by an FTIR spectrometer. Figure 25 shows the observed spectrum at various temperatures.
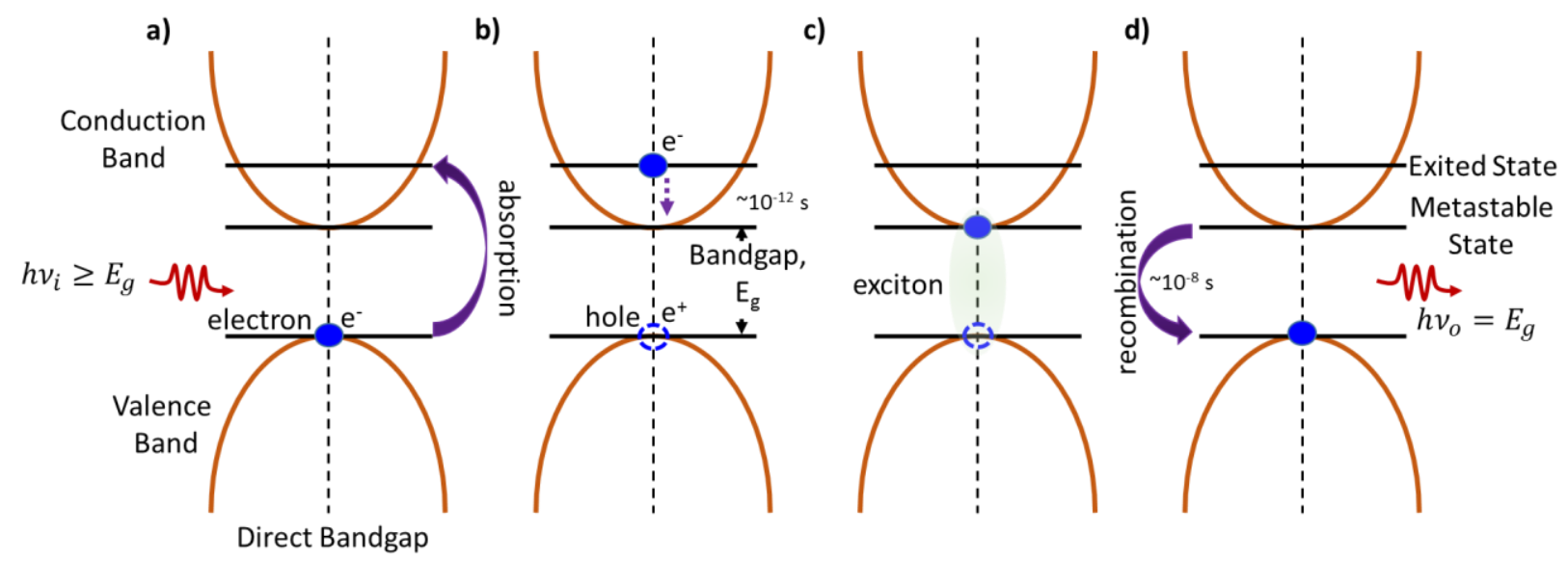

Figure 23. Graphical depiction of photoluminescence process in a direct bandgap semiconductor: a) electrons are excited to a state above the bottom of the conduction band upon absorption of pump photons;

$b)$ the electron undergoes non-radiation relaxation down to the bottom of the conduction band; $c)$ an exciton is created between the electron at the bottom of the conduction band and holes at the top of the valence band; $d$ ) the electron relaxes from the metastable state recombining with the hole and the atom emits a photon with an energy of $E_{g}$. 


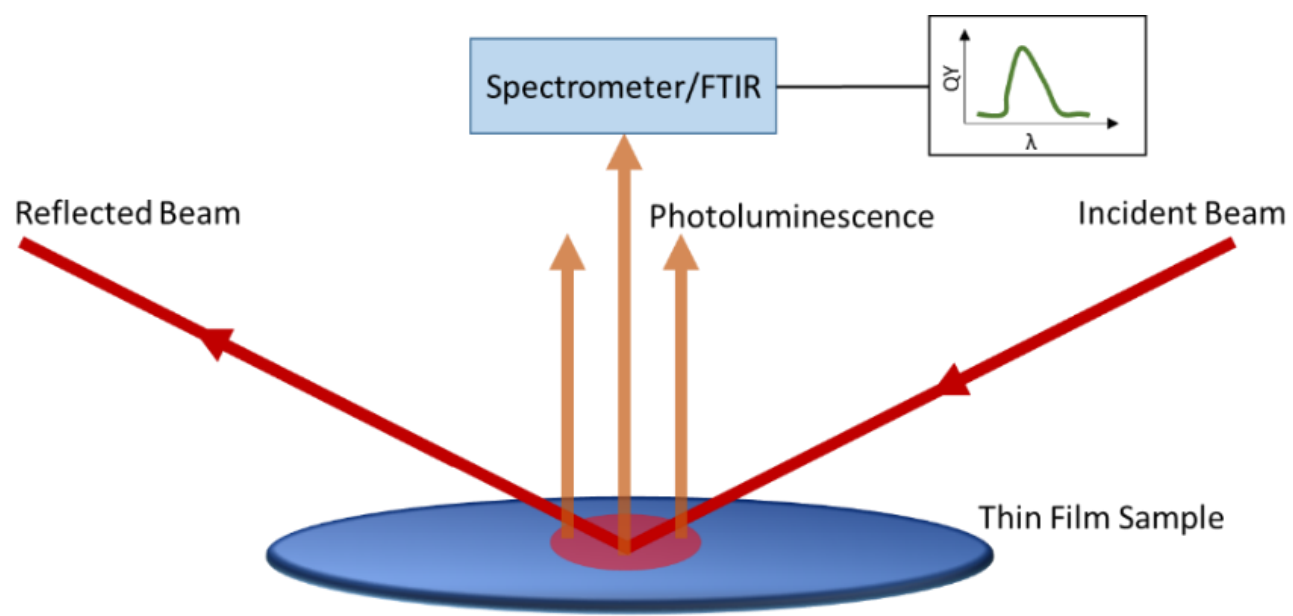

Figure 24. Graphical depiction of a photoluminescence setup.

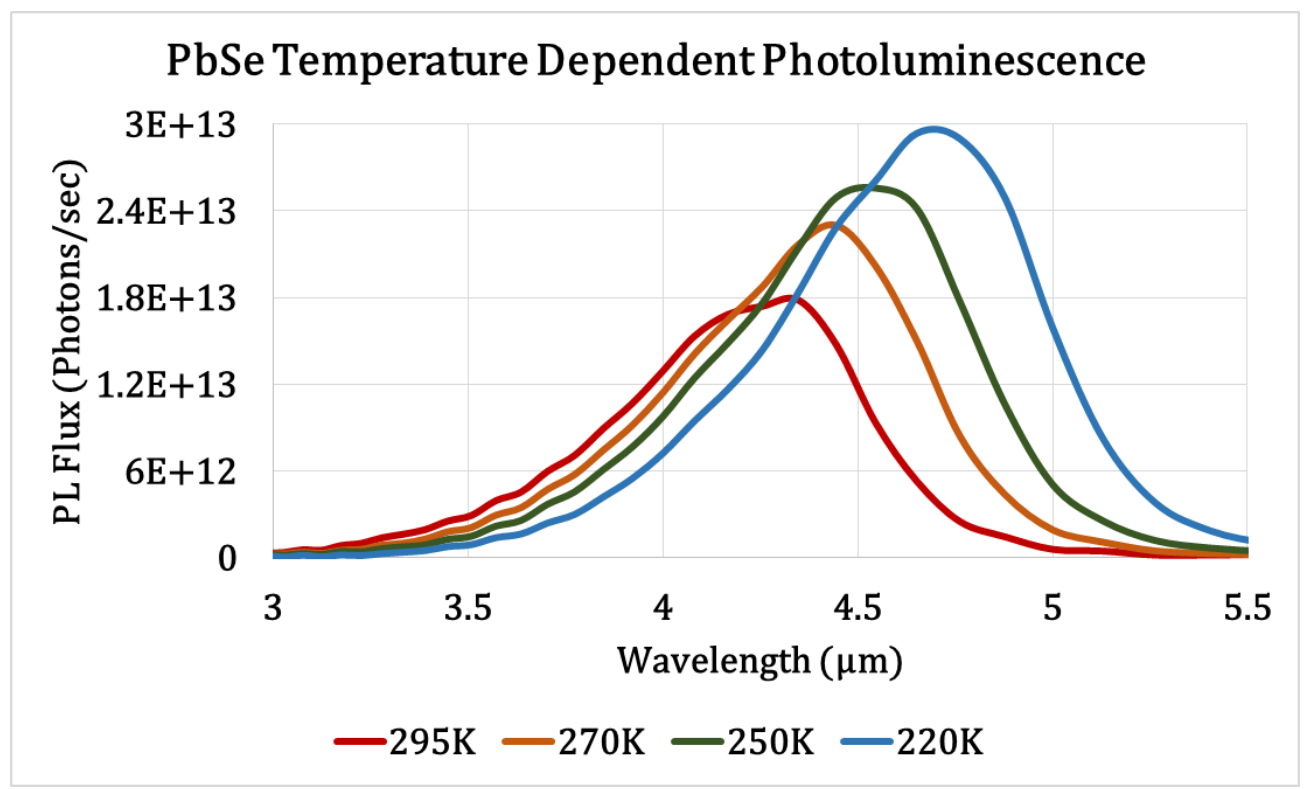

Figure 25. Measured temperature dependent photoluminescence of sensitized PbSe films.

Described above, the peak of the PL spectrum is associated with the bandgap energy, while the amplitude is the emitted photon flux. The results in figure 25 are redshifted, yet still show good agreement with those presented in [36]. This redshift can be quantified by dividing the peak energy or wavelength value at a given temperature by that at room temperature. Table 4 contains red-shift scaling factors, which were utilized to estimate how the dispersion relations change with temperature. Also of note in figure 25 
is the inverse relationship between emitted photon flux and temperature. Discussed above, this trend follows the same behavior observed in the responsivity and quantum efficiency of fabricated PbSe detectors.

Table 4. Temperature dependent scaling factor calculated from the PL spectra of investigated PbSe films.

\begin{tabular}{|c|c|c|c|}
\hline Temperature (K) & $\begin{array}{c}\text { Max Flux } \\
\text { (Photons/s) }\end{array}$ & $\begin{array}{c}\text { Eg Wavelength } \\
(\boldsymbol{\mu m})\end{array}$ & $\begin{array}{c}\text { Red-shift Scaling } \\
\text { Factor }\end{array}$ \\
\hline 290 & $1.74 \mathrm{e} 13$ & 4.256 & 1 \\
\hline 270 & $2.30 \mathrm{e} 13$ & 4.445 & 1.044 \\
\hline 250 & $2.56 \mathrm{e} 13$ & 4.546 & 1.068 \\
\hline 230 & $2.81 \mathrm{e} 13^{*}$ & $4.647^{*}$ & $1.092^{*}$ \\
\hline 220 & $2.81 \mathrm{e} 13$ & 4.652 & 1.093 \\
\hline *Estimated from surrounding temperature values
\end{tabular}

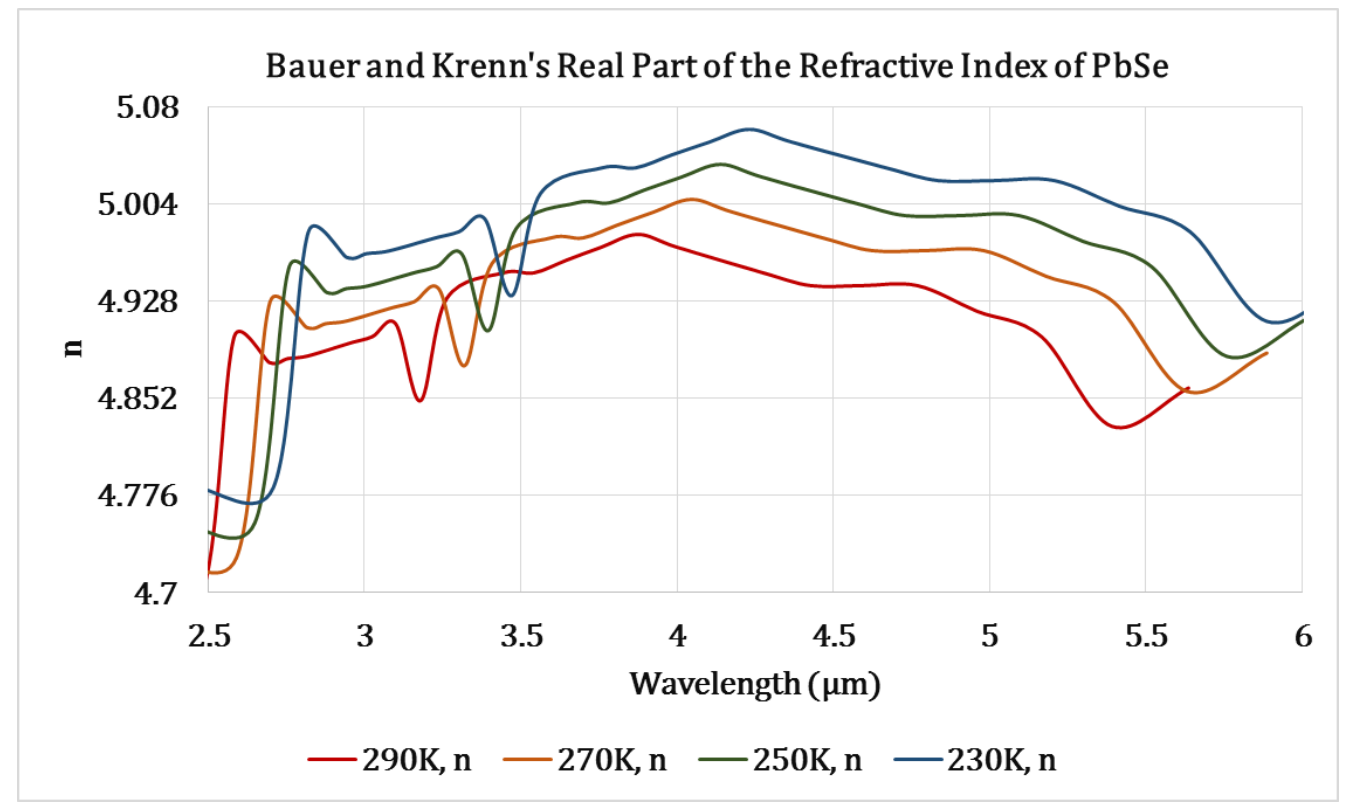

Figure 26. The real part of the index of refraction of PbSe from $2.5 \mu \mathrm{m}$ to $6 \mu \mathrm{m}$ at temperatures of interest.

To estimate how the amplitude of the complex index of refraction changes with temperature, the real part and the imaginary part must be considered separately. The real part of the index of refraction changes with temperature $\mathrm{T}$ according to a constant of proportionality called $\mathrm{dn} / \mathrm{dT}$, which for $\mathrm{PbSe}$ was found in the literature to be 


$$
\frac{1}{n_{0}}\left(\frac{d n}{d T}\right)=-28 \times 10^{-5} K^{-1}
$$

where $\mathrm{n}_{0}$ is defined as the index of refraction at room temperature [35]. From equation 2.16, the dispersion relations of table 3, and the red-shift scaling factors in table 4 the real part of the index of refraction between $2.5 \mu \mathrm{m}$ and $6 \mu \mathrm{m}$ was calculated. The resulting temperature dependence of the real part of the index of refraction from $290 \mathrm{~K}$ down to $230 \mathrm{~K}$ is shown in figure 26.

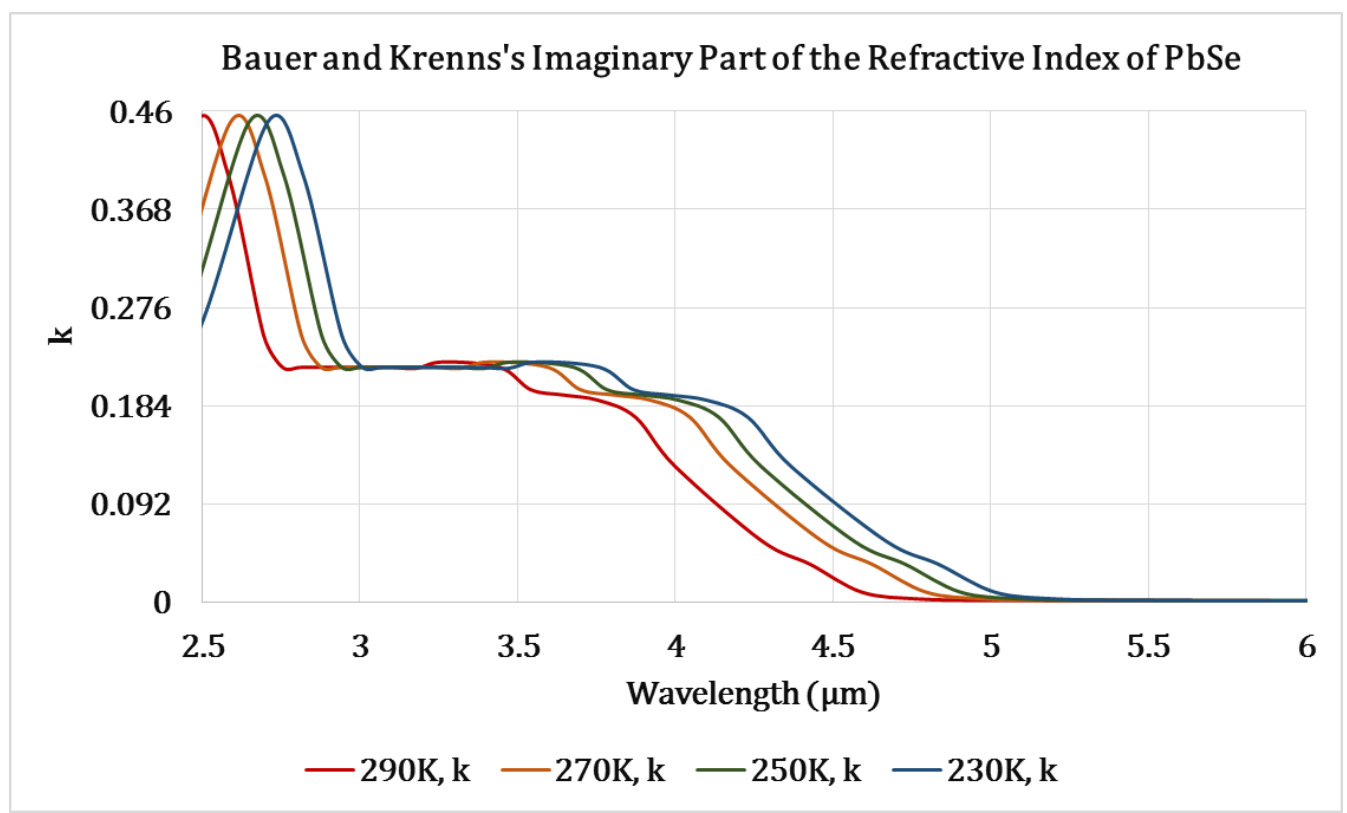

Figure 27. The imaginary part of the index of refraction of PbSe between $2 \mu m$ and $6 \mu \mathrm{m}$ at the temperatures of interest.

A first assumption for the imaginary part of the index of refraction might be to consider figure 22 , and observe that the response increases as the temperature decreases. Therefore, the imaginary part must increase. However, if this assumption were to be true, the absorption would also increase. Instead, consider the effect of temperature on the carrier concentration shown in table 2 . The thermal energy dependence would suggest that the recombination rate should decrease, therefore increasing the performance of the 
detector. The increase in performance in the material relates to a quantity known as the internal quantum efficiency, which is defined as the ratio of the number of carriers that contribute to photocurrent to the number of carriers generated upon photoexcitation [13]. The external quantum efficiency shown in figure 22 can also be defined as the product of the internal quantum efficiency and the absorption of the semiconductor material.

Therefore, the assumption for this study is that the absorption remains constant, while the internal quantum efficiency is inversely proportional to temperature. The temperature dependence of the imaginary part of the index of refraction for PbSe according to Bauer and Krenn's dispersion relations and the red-shift scaling factors in table 4 are shown in figure 27.

When comparing the EQE of figure 22 and the k-values of figure 27, the overall shape of the response should be the same. In figure 22 the cutoff wavelength is approximately $4.6 \mu \mathrm{m}$. Bauer and Krenn's dispersion relations, however, have a cutoff at room temperature around $4.25 \mu \mathrm{m}$ [33]. Their dispersion relations suggest a cutoff closer to the bandgap energy of $0.29 \mathrm{eV}(4.27 \mu \mathrm{m})$ of $\mathrm{PbSe}$, however the measured absorption from the films shows a 1.08x redshift in the cutoff wavelength. Recall, however that the investigated films herein have a three-layer polycrystalline structure. Therefore, the discrepancy is associated with additional absorptive states within the bandgap leading to what are known as mid-gap states and the Urbach tail [40-41]. When an electron near the top of the valence band is excited to an energy state close to the conduction band in a disordered material, it can experience thermal fluctuations that blur the well-defined edges of both bands. This blurring leads to additional energy states, as well as an exponential tail that has effects on the absorption coefficient $\alpha$. Considering these films 
are polycrystalline and highly disordered, I shifted the wavelength of Bauer and Krenn's dispersion relations by taking the cutoff wavelength of the measured absorption and dividing by the cutoff wavelength they report. The resulting dispersion relations are shown in figure 28 . The cutoff and knee wavelengths at $290 \mathrm{~K}$ are now in agreement with the measured absorption spectra of figure 20 .

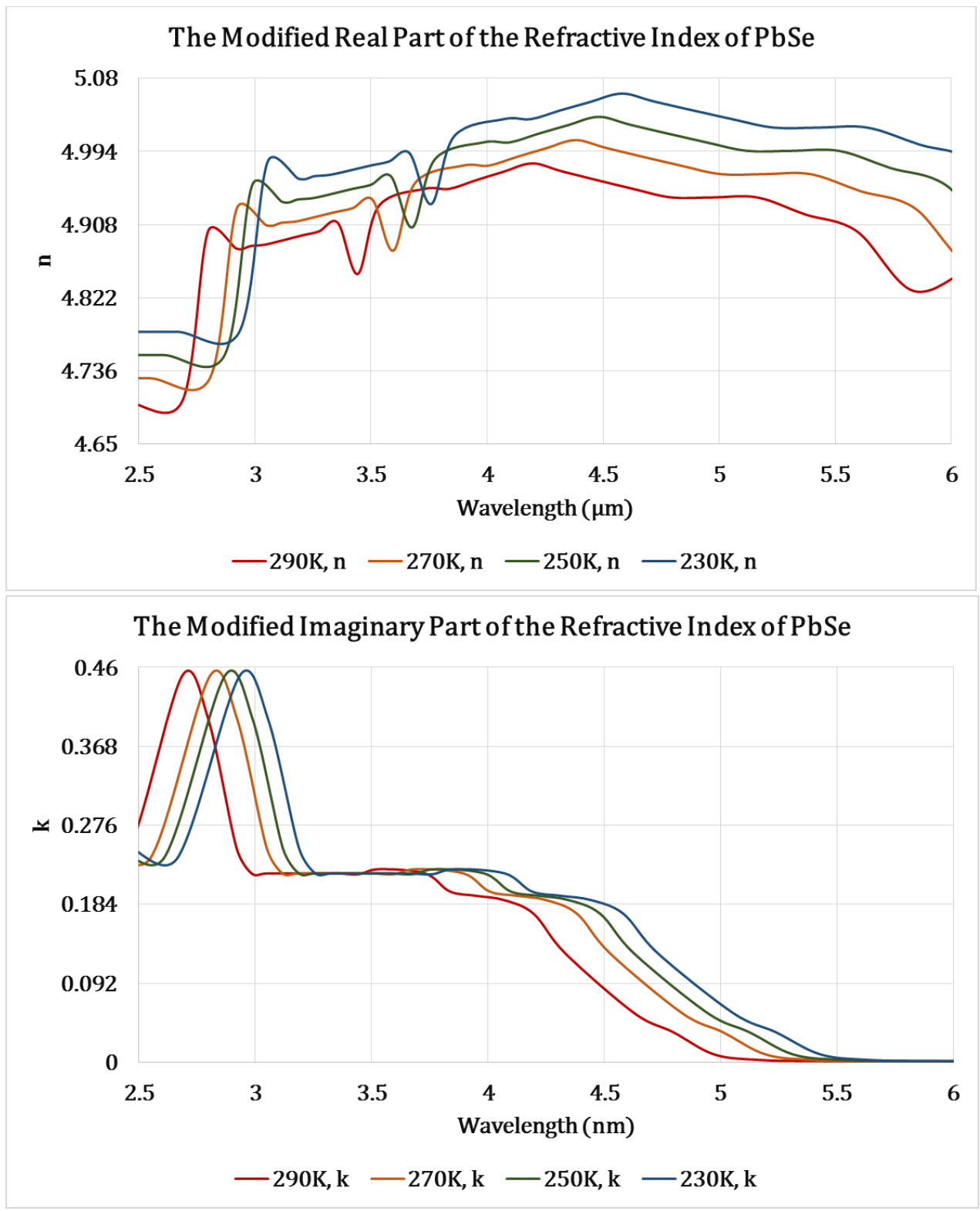

Figure 28. Modified dispersion relations of PbSe. 
There are several important features to acknowledge in figure 28 . The k-values have been kept constant across temperature to be consistent with the assumption that absorption should be approximately constant across temperatures. Further, the shape of the k-value spectra are similar to the measured EQE spectra, which is expected because $\mathrm{EQE}$ is dependent on the absorption within the material. Finally, for resonant structures, especially with respect to surface plasmon resonance and resonant cavities, the index of the host material is one of the primary parameters that governs the resonant behavior [ 31 , 42]. These modified dispersion relations that describe the PbSe films we investigated have been used extensively in numerical and finite element modeling of films with embedded resonant structures to predict the optical parameters, device performance, and theoretical absorption enhancement.

Recall from figure 3 that the baseline structure of the PbSe films discussed herein (from bottom up) is $\mathrm{Si}$ substrate, $\mathrm{SiO}_{2}, \mathrm{PbSe}$, and $\mathrm{PbI}_{2}$. The dispersion relations of $\mathrm{PbSe}$ have already been discussed, however the other dispersion relations must also be known to accurately model and predict the optical properties of the films. To ensure the modeled absorption was only from $\mathrm{PbSe}$, the k-values for $\mathrm{Si}, \mathrm{SiO}_{2}$, and $\mathrm{PbI}_{2}$ were excluded from this investigation. This assumption closely approximates the real system, because all three compounds are wide bandgap materials and therefore transparent in the band of interest. The index of refraction of $\mathrm{Si}$ is approximately 3.45 , while the index of refraction of $\mathrm{SiO}_{2}$ is approximately 1.4 in the MWIR band [43-44]. The real part of the index of refraction of $\mathrm{PbI}_{2}$ is between 2.3 and 2.5 in the MWIR regime [11, 45-46], so for this study $\mathrm{PbI}_{2}$ was taken to be 2.5 . In these films, the index of refraction alternates from lower to higher values multiple times as light propagates through them. As I have 
previously discussed in Chapter 1 , this will cause constructive and destructive interference fringes within the film which can be tailored to selectively enhance the inband performance of $\mathrm{PbSe}$. 


\section{Chapter 2 References}

[1] S. -S., Yoo, et al. "High Operating Temperature MWIR Photodetectors Development Using Polycrystalline PbSe Technology," presented at The U.S. Workshop on the Physics and Chemistry of II-VI Materials, Chicago, IL, 2017.

[2] M. - H., Jang, ““'Effect of Sensitization on PbSe IR Detector Materials Properties." Manuscript submitted to J. of Elect. Mat., 2017.

[3] B. Hafner, "Energy Dispersive Spectroscopy on the SEM: A Primer," University of Minnesota [online], Available: http://www.charfac.umn.edu/instruments/eds_on_sem_primer.pdf [accessed Oct. 30, 2018].

[4] R. S. Mitchell, "Structural polytypism of lead iodide and its relationship to screw dislocations.” Z. Kristallogr. Cryst. Mater. Vol. 111, Iss. 1-6, 1959.

[5] Z. I. Smirnova, et al. "Effect of an Iodine-Containing Additive on the Composition, Structure, and Morphology of Chemically Deposited Lead Selenide Films." Physics of the Solid Stat, Vol. 56, No. 12, 2014.

[6] Y. Suh and S. - H. Suh, "Effect of iodine doping in the deposition solution and iodine vapor pressure in the sensitization treatment on the properties of $\mathrm{PbSe}$ films." Optical Engineering, Vol. 56, No. 9, 2017.

[7] J. Grayer, P. Martin, M. Kramer, and S. -S. Yoo, "Material and Device Characterization of High-Temperature PbSe Photoconductive films," presented at Military Sensing Symposia, Gaithersburg, MD. 2018

[8] S. Horn, et al. "Reaching for the sensitivity limits of uncooled and minimally cooled thermal and photon infrared detectors.” Proc. Of SPIE, Vol. 5783, 2005. 
[9] M. Kramer, et al. "Effect of Temperature on the Sensitization of Lead Selenide," presented at the Northrop Grumman Corporation Materials Forum, Rolling Meadows, IL. 2017.

[10] D. A. Neamen, Semiconductor Physics and Devices: Basic Principles, 3rd Ed, New York, NY: McGraw Hill, 2003.

[11] R. Ahuja, et al. "Electronic and Optical Properties of Lead Iodide." J. Appli. Phys., Vol. 92, No 12, 2002.

[12] J. N. Humphrey and R. I. Petritz, "Photoconductivity of Lead Selenide: Theory of the Mechanism of Sensitization." Physical Review, Vol. 105, No. 6, 1957.

[13] J. Singh, Optoelectronics: An Introduction to Materials and Devices," New York: McGraw-Hill Companies, Inc. 1996.

[14] P. R. Norton, "Photodetectors," in McGraw-Hill's Handbook of Optics, 3rd ed., Vol. 2, New York: McGraw-Hill, 2010, pp. 24.3-24.100.

[15] Lihuazhao, et al. "Understanding sensitization behavior of lead selenide photoconductive detectors by charge separation model.” J. of Appl. Phys., Vol. 115, 084502, 2014.

[16] W. V. Roosebroeck, "Current-Carrier Transport and Photoconductivity in Semiconductors with Trapping,” Physical Review, Vol. 119, No. 2, 1960.

[17] M. P. Marder. Condensed Matter Physics, 2nd ed. Hoboken, NJ: John Wiley and Sons, Inc. 2010.

[18] V. D. Mihailetchi, J. Wilderman, and P. W. M. Blom, "Space-Charge Limited Photocurrent," Physical Review Letters, Vol. 94, 126602, 2005. 
[19] Y. Nishina and G. C. Danielson, "Measurements of Minority Carrier Lifetimes in Semiconductors,” Ames Laboratory ISC Technical Report ISC-926, 1957.

[20] W. K. Metzger, et al. "Time-Resolved Photoluminescence and Photovoltaics," presented at the DOE Solar Energy Technologies Program Review, Denver, CO. 2004.

[21] S. Dunford. "Calculating the Time Constant of an RC Circuit," Undergraduate Journal of Mathematical Modeling: One+2, Vol. 2, Iss. 2, Art. 3, 2010.

[22] R. K. Ahrenkiel, “Analysis of Techniques for Measuring Carrier Recombination Lifetime," presented at the US Department of Energy Conference on Energy Efficiency and Renewable Energy, 2013.

[23] P. Kuman, et al. "PbSe Mid-IR Photoconductive Thin Films (Part 1): Phase Analysis of the Functional Layer," Journal of Alloys and Compounds, Vol. 724, 2017.

[24] Thermo Scientific, Advanced FT-IR spectroscopy: Principles, Experiments, and Applications, Thermo Scientific Instruments, LLC, 2012.

[25] P. R. Griffiths and J. A. Haseth, Fourier Transform Infrared Spectrometry, $2^{\text {nd }}$ ed, Hoboken, NJ: John Wiley \& Sons, Inc. 2007.

[26] T. A. Blake, et al. "Methods for quantitative infrared directional-hemispherical and diffuse reflectance measurements using an FTIR and commercial integrating sphere," Applied Optics, Vol. 57, No. 3, 2018.

[27] M. Falk and A. G. Miller, "Infrared spectrum of carbon dioxide in aqueous solution," Vibrational Spectroscopy, Vol. 4, Iss. 1, 1992. 
[28] M. Falk and T. A. Ford, "Infrared Spectrum and Structure of Liquid Water," presented at the Symposium on Molecular Structure and Spectroscopy, Columbus, OH, 1965.

[29] B. Shokri, M. A. Firouzjah, and S. I. Hosseini, "FTIR analysis of silicon dioxide thin film deposited by metal organic-based PECVD," presented at the $19^{\text {th }}$ International Symposium on Plasma Chemistry, 2009.

[30] Thor Labs "FDPSE2x2 Lead Selenide Photoconductor User Guide," Rev C, pg 8, 2017.

[31] E. Hecht, Optics, $4^{\text {th }}$ ed. New York: Addison-Wesley, 2002.

[32] I. Chambouleyron and J. M. Marinez, Handbook of Thin Film Materials; ch 12, edited by H.S. Nalwas, Academic Press, 2001.

[33] J. H. W. G. Boer, Spectroscopic infrared ellipsometry: components, calibration and application, Eindhover: Technische Universiteit Eindhoven, 1995.

[34] G. Bauer and H. Krenn, "Lead Selenide (PbSe)", in Palik, E.D. Editor, Handbook of Optical Constants of Solids, (Vol. 1, pp. 517 - 523). San Diego, CA. United States: Academic Press, Inc. 1985.

[35] S. Adachi, Optical Properties of Crystalline and Amorphous Semiconductor: Materials and Fundamental Principles, New York, New York: Springer, 2012.

[36] N. M Ravindra, S. Auluck, and V. K. Srivastava, "Temperature dependence of the energy gap in PbS, PbSe, and PbTe,” Phys. Sat. Sol. (a) 52, K151, 1979.

[37] Z. Dashevsky, et al. "Mid-infrared photoluminescence of PbSe film structures up to room temperature," Proc. Of SPIE, Vol. 7142, 71420L-1, 2008. 
[38] K. Bhatnagar, "Nanogap Embedded Silver Gratings for Surface Plasmon Enhanced Fluorescence,” M.S. Thesis, University of Missouri, Columbia, MO, 2012.

[39] J. R. Laokowicz, Principles of Fluorescence Spectroscopy, $3^{\text {rd }}$ ed. Springer, 2006.

[40] A. Lukyanov, et al. "Structural origin of the midgap electronic states and the Urbach tail in Pnictogen-Chalcogenide glasses," J. Phys. Chem. B, 2018.

[41] Sadigh, et al. "First-principles calculation so of the Urbach tail in the optical absorption spectra of silica glass," Phys. Rev. Lett. PRL 106, 027401, 2011.

[42] D. Sarid and W. Challner, Modern Introduction to Surface Plasmons: Theory, Mathematical Modeling, and Applications, New York, NY: Cambridge University Press, 2010.

[43] H. H. Li, "Refractive index of silicon and germanium and its wavelength and temperature derivatives.” J. Phys. Chem. Ref. Data 9, 1993.

[44] J. Kischkat, et al. "Mid-infrared optical properties of thin films of aluminum oxide, titanium dioxide, silicon dioxide, aluminum nitride, and silicon nitride." Appl. Opt. 51, 2012.

[45] A. E. Dugan and H. K. Henisch, "Dielectric Properties and Index of Refraction of Lead Iodide Single Crystals". J. Phys. Chem. Solids. Pergamon Press, Great Britain, 1967.

[46] L. Glasser, et al. "Dielectric Properties of Lead Iodide". J. Chem. Soc. (A), 1967.

[47] Power Technology, Inc., "IQ1C100(LD2191)," 2018. [Online]. Available: https://www.powertechnology.com/b071.html. [Accessed; Nov. 9, 2018]. 
[48] D. A. Kondas, "Introduction to Lead Salt Infrared Detectors," Technical Report ARFSD-TR-92024, US Army Armament Research, Development, and Engineering Center, 1993.

[49] D. J. Lovell, "The Development of Lead Salt Detectors," American Journal of Physics, Vo. 37, No. 2, 1957.

[50] G. Vergara, et al. "Polycrystalline lead selenide: the resurgence of an old infrared detector," Opto-Electronics Review, 15(2), 110-117, 2007. 


\section{Chapter 3: Surface Plasmon Resonance}

\subsection{Electrodynamics and Light-Matter Interaction}

In Chapter 1, surface plasmons and surface plasmon resonance was defined without derivation. Hence, the electrodynamic relationships for surface plasmons are discussed in detail in this chapter. Equations 3.1-3.4 show the familiar Maxwell's equations in differential form [1-4].

$$
\begin{gathered}
\nabla \cdot \boldsymbol{D}=\rho \\
\nabla \cdot \boldsymbol{B}=0 \\
\nabla \times \boldsymbol{E}=-\frac{\partial \boldsymbol{B}}{\partial t} \\
\nabla \times \boldsymbol{H}=\boldsymbol{J}+\frac{\partial \boldsymbol{D}}{\partial t}
\end{gathered}
$$

Equation 3.1, Gauss's law, relates the electric flux density $\mathbf{D}$ to the charge density $\rho$. Equation 3.2 is Gauss's law of magnetism, where $\mathbf{B}$ is the magnetic flux density, which states that there are no magnetic monopoles. Equation 3.3, Faraday's law, shows that a change in the electric field $\mathbf{E}$ induces a change in $\mathbf{B}$ and vice versa. Finally, Ampere's law, from equation 3.4, shows that a change in the magnetic field $\mathbf{H}$ is related to the change in $\mathbf{D}$, in addition to the electric current density $\mathbf{J}$.

Maxwell's equations are not complete without the constitutive relationships, given in equations $3.5-3.7$,

$$
\begin{gathered}
\boldsymbol{D}=\varepsilon \boldsymbol{E}=\varepsilon_{0} \varepsilon_{r} \boldsymbol{E} \\
\boldsymbol{B}=\mu \boldsymbol{H}=\mu_{0} \mu_{r} \boldsymbol{H} \\
\boldsymbol{J}=\sigma \boldsymbol{E}
\end{gathered}
$$


where $\varepsilon_{0}$ is the vacuum permittivity which is equal to $8.854 \times 10^{-12} \mathrm{~F} / \mathrm{m}, \varepsilon_{r}$ is the relative dielectric constant of the material, $\mu_{0}$ is the vacuum permeability which is equal to $4 \pi \times 10^{-7} \mathrm{H} / \mathrm{m}, \mu_{r}$ is the relative permeability of the medium assumed to be one for all calculations, and $\sigma$ in Siemens/cm is the specific conductivity which represents power loss in a material. The discussion of surface plasmons deals with the phenomena that occur at the interface between dielectric and metallic media. Therefore, we must consider the boundary conditions for solving Maxwell's equations [4].

\subsection{Surface Plasmon Polaritons}

Consider a spherical metal particle located on the surface of a dielectric medium. Light incident on the metallic particle will either be reflected, scattered and diffracted, or absorbed by this metallic surface feature [4-5]. Here, a distinction has been made between reflection (back scattering) and forward scattering, but in general scattering and reflection are synonymous. If the frequency of the incident light is such that it meets the plasmonic resonance condition, then either radiative or non-radiative evanescent waves will be induced at the interface between the dielectric and metallic particle [4-6]. If scattering and diffraction dominate, then evanescent waves will radiate and decay from the interface into the near field of the dielectric [4-5].

\subsubsection{Spherical Particles in the Quasi-Static Limit}

In the limit that the radius of the particle is much smaller than the wavelength of incident light $(r \ll \lambda)$, a classical approach can be employed for determining the surface

plasmon (SP) resonance of a material system [7-8]. In the classical limit, the response of surface plasmons is due to induced polarization of the metal particle by an external 
driving field [4-5, 7-10]. Moreover, the incident light can be treated as a plane wave, and the metallic particle can be treated as a dipole oscillating with a resonant frequency $\omega_{p}$ known as the plasma frequency.

\subsubsection{Drude-Lorentz Model of Metals}

To discuss the metallic particle, we draw on the Drude-Lorentz, also known as the free-electron, model [4-6, 11]. For this model, the conduction electrons in a metal are treated as a gas of free, non-interacting carriers that undergo relaxation through collisions with the metal lattice and other scatterers [4-6]. We can describe the dynamics of these free electrons using Newton's equation of motion shown in equation 3.8,

$$
m \frac{d^{2} x}{{d t^{2}}^{2}}=-\frac{m}{\tau} \frac{d x}{d t}-q E_{t o t}(t)
$$

where $\mathrm{m}$ is the effective electron mass in the metal, $\mathrm{x}$ is the displacement distance during oscillation, q denotes the electric charge constant, and $\tau$ is the decay time which accounts for electron scattering. The term $\mathrm{E}_{\text {tot }}$ represents the total electric field, including the incident and induced fields acting on the electrons in the metal, and is given by equation 3.9 .

$$
E_{t o t}(t)=\operatorname{Re}\left\{E_{t o t}(\omega) \exp (-i \omega t)\right\}
$$

It is important to note how the interaction between the metal and the incident field is dependent on the field frequency $\omega$. The spatial displacement of the electrons during oscillation is also a function of frequency, and is given by equation 3.10.

$$
x(\omega)=\frac{q E_{t o t}(\omega)}{m\left(\omega^{2}+\frac{i \omega}{\tau}\right)}
$$


Assuming a 2D density of states and an electron density of $\mathrm{N}$, the polarization $\mathrm{P}$ induced in the metal particle by the incident light is given by equation 3.11 .

$$
P(\omega)=-q x(\omega) N
$$

Recall from equation 3.1 that the electric flux density is a product of the permittivity and the electric field in metals. Using the Drude-Lorentz model, the magnitude of the electric flux density in a metal is given by equation 3.12 .

$$
D(\omega)=\varepsilon(\omega) E_{t o t}(\omega)=\varepsilon_{\infty}(\omega) E_{t o t}(\omega)+4 \pi P(\omega)
$$

The above relationship leads to the metal permittivity, shown in equation 3.13 , as described in the Drude-Lorentz model. This relationship is needed to describe the surface plasmon resonance condition [4-6, 7-10].

$$
\varepsilon(\omega)=\varepsilon_{\infty}(\omega)-\frac{\omega_{p}^{2}}{\omega^{2}+\frac{i \omega}{\tau}}
$$

The first term on the right hand side of equation $3.13, \varepsilon_{\infty}$, represents the high-frequency permittivity limit of the metal, and $\omega_{p}$ is the bulk plasma frequency of the metal given by equation 3.14.

$$
\omega_{p}=\sqrt{\frac{4 \pi q^{2} N}{\epsilon_{\infty} m}}
$$

It is important to remark on the time constant $\tau$, and how it relates to SPs at this point. Notice how if $\tau$ approaches infinity, then the denominator of the right side is only a function of the frequency of the incident light. This implies the surface plasmons have an infinite lifetime, and will have sharp resonances at the plasma frequency. However, if $\tau$ is finite, then plasmon resonances become broadened around a specific frequency that 
depends on the surrounding material and the plasma frequency of the metal [4]. The surface plasmon resonance lifetimes of various metals will be revisited shortly.

For completeness, we can now revise equations 3.9 and 3.10 to include the contributions from the external $E_{\text {ext }}$ and induced fields. The total electric field and spatial displacement of electrons in a metal due to an external field is shown in equations 3.15 and 3.16.

$$
\begin{gathered}
\mathrm{E}_{\mathrm{tot}}=\mathrm{E}_{\text {ext }}(\omega)+\frac{4 \pi N \mathrm{Na}(\omega)}{\varepsilon_{\infty}} \\
x(\omega)=\left(\omega^{2}+\frac{i \omega}{\tau}-\frac{4 \pi N q^{2}}{\varepsilon_{\infty} m}\right)=\frac{q}{m} E_{\text {ext }}(\omega)
\end{gathered}
$$

\subsubsection{Metal Spheres in the Classical Limit}

From the classical or quasi-static treatment a metallic particle can be treated as a

dipole oscillator with a resonant frequency $\omega_{p}[4-5]$. Here, the incident light is considered to be a plane wave with a uniform field distribution. In other words, we will ignore the spatial distribution of intensities of the incident field, and only consider the time dependence.

In the quasi-static limit, we can use equation 3.17, Laplace's equation, for the static potential to solve for the electric field in spherical coordinates given in equation 3.18 .

$$
\begin{gathered}
\nabla^{2} \Phi=0 \\
\mathbf{E}=-\nabla \Phi
\end{gathered}
$$


If the incident plane wave is polarized along the $\mathrm{x}$-direction, then the lowest order solution for the total field is that of an induced dipole [4]. Inside the metal sphere, the electric potential is given by equation 3.19 ,

$$
\Phi_{\text {in }}=A_{p} x
$$

where $A_{p}$ is the amplitude of the potential, and $\mathrm{x}$ is the displacement. Therefore, the electric field inside the metal sphere is given by equation 3.20 [4-5].

$$
\boldsymbol{E}_{\text {in }}=-A_{p} \hat{x}=-A_{p}(\hat{r} \sin \theta \cos \varphi+\hat{\theta} \cos \theta \cos \varphi-\hat{\varphi} \sin \varphi)
$$

Outside the sphere, the field potential, shown in equation 3.21, is given by the sum of the contributions from the external and induced dipole fields,

$$
\Phi_{\text {out }}=-\mathrm{Ex}+\frac{\mathrm{C}_{\mathrm{DP}}}{\mathrm{r}^{2}} \sin \theta \cos \varphi
$$

where $\mathrm{r}$ is the radius of the spherical particle, and $\mathrm{C}_{\mathrm{DP}}$ is the amplitude of the dipole potential. Using equation 3.18 and 3.21 , the electric field outside of the particle is given by equations 3.22 and 3.23 .

$$
\begin{gathered}
\boldsymbol{E}_{\text {out }}=E \hat{x}-C_{D P}\left[\frac{\hat{x}}{r^{3}}-\frac{3 x}{r^{5}}(x \hat{x}+y \hat{y}+z \hat{z})\right] \\
\boldsymbol{E}_{\text {out }}=E(\hat{r} \sin \theta \cos \varphi+\hat{\theta} \cos \theta \cos \varphi-\hat{\varphi} \sin \varphi)+ \\
\frac{C_{D P}}{r^{3}}(2 \hat{r} \sin \theta \cos \varphi-\hat{\theta} \cos \theta \cos \varphi+\hat{\varphi} \sin \varphi)
\end{gathered}
$$

Due to the fact that the host material and metallic sphere have different dielectric properties, there will be an induced charge that perturbs the incident electric field. Shown in equation 3.24 and 3.25 , there is continuity across the sphere boundary between the tangential component of the electric field $E_{t}$ and the normal component of the displacement field $E_{n}$, respectively, 


$$
\begin{gathered}
E_{t_{\text {outside }}}=E_{t_{\text {inside }}} \\
\mathrm{E}_{\mathrm{n}_{\text {outside }}}=\frac{\varepsilon_{\mathrm{m}}}{\varepsilon_{\mathrm{d}}} \mathrm{E}_{\mathrm{n}_{\text {inside }}}
\end{gathered}
$$

where $\varepsilon_{m}$ is the frequency dependent relative permittivity of the metal sphere, and $\varepsilon_{d}$ is the relative permittivity of the host dielectric material, which is assumed to be constant in the quasi-static model [5]. Therefore, we can relate the static $A_{p}$ and induced $C_{D P}$ amplitudes using equations 3.26 and 3.27.

$$
\begin{gathered}
A_{p}=-\left(\frac{3 \varepsilon_{d}}{2 \varepsilon_{d}+\varepsilon_{m}}\right) E \\
C_{D P}=\frac{r^{3}\left(\varepsilon_{m}-\varepsilon_{d}\right)}{2 \varepsilon_{d}+\varepsilon_{m}} E
\end{gathered}
$$

The relative dielectric constant of the metal, shown in equation 3.28, has both a real and imaginary part, which relate to the phenomena of scattering and absorption.

$$
\varepsilon_{m}=\varepsilon_{m}^{\prime}+i \varepsilon_{m}^{\prime \prime}
$$

Equations 3.26 and 3.27 lead to the polarizability of the metal, which is defined as the ability for carriers within the metal to align into dipoles. Shown in equation 3.29 , the polarizability $\alpha$ is highly dependent on the complex permittivity of the metal and the host material.

$$
\alpha(\omega)=\frac{4 \pi \varepsilon_{0} \varepsilon_{\mathrm{d}} \mathrm{C}_{\mathrm{DP}}}{\mathrm{E}}=\left(4 \pi \varepsilon_{0} \varepsilon_{\mathrm{d}}\right) \mathrm{r}^{3}\left(\frac{\varepsilon_{\mathrm{m}}-\varepsilon_{\mathrm{d}}}{2 \varepsilon_{\mathrm{d}}+\varepsilon_{\mathrm{m}}}\right)
$$

Notice, that as the denominator in equation 3.29 goes to zero, then the polarizability approaches infinity, and the plasmonic resonance condition for spherical metallic particles is reached [4-10]. In real metals, the polarizability would approach a maximum, yet still adhere to the same resonance condition shown in equation 3.30 [34-35]. 


$$
\varepsilon_{m}^{\prime}(\omega) \cong-2 \varepsilon_{d}
$$

Equation 3.30 states that the surface plasmon resonance condition for spherical particles occurs when the frequency of the incident light is such that the real part of the complex permittivity of the metal is negative, with an amplitude that is two-times that of the surrounding dielectric $[4-10,21-22,30,36]$. Notice the resonance condition in the classical limit is independent of size. The size independence is due to the omission of retardation effects in the metal [4-5]. As will be discussed shortly this is not the case for larger particles, and resonances will shift to lower frequencies as particle sizes increase $[5,7,12-15]$. Also of note for the quasi-static limit is that we assume the incident light only couples to dipole resonances [4-5]. However, when the theory is expanded into the Mie regime, light can also couple to multi-pole resonances.

To get a glimpse of how surface plasmons can greatly increase the field strength of the incident light, consider the peak field intensity. The spatial location of the peak field intensity will be located where the sphere and the dielectric medium meet $(\varphi=$ $0, \theta=\pi / 2$ ). At resonance, the electric field outside the sphere is given by equation 3.31.

$$
\boldsymbol{E}_{\text {out }}=\left[1+\frac{2\left(\varepsilon_{m}-\varepsilon_{d}\right)}{2 \varepsilon_{d}+\varepsilon_{m}}\right] E \hat{r}=\left[\frac{3 \varepsilon_{m}^{\prime}}{i \varepsilon_{m}^{\prime \prime}}\right] E \hat{r}
$$

The imaginary part of equation 3.31 represents the losses in the metal, so for metals in which the losses are small $\left(\left|\varepsilon_{m}^{\prime}\right| \gg\left|\varepsilon_{m}^{\prime \prime}\right|\right)$, the maximum predicted field enhancement at resonance on the surface of the particle is given by equation 3.32 [4],

$$
\left|\frac{E_{S P}}{E_{0}}\right| \cong 3\left|\frac{\varepsilon_{m}^{\prime}}{\varepsilon_{m}^{\prime \prime}}\right|
$$

where $E_{s p}$ represents the field on the metallic sphere surface due to surface plasmons, and $\mathrm{E}_{0}$ represents the incident field. The relationship in equation 3.32 is a first approximation 
for how large the electric field at the surface of a metallic sphere in a host material can be. Consider the carrier generation rate $\mathrm{G}_{\mathrm{L}}$, with units of $\mathrm{cm}^{-3} \mathrm{~s}^{-1}$, for photoconductors shown in equation 3.33,

$$
G_{L}=\left(1-e^{-\alpha x}\right) I_{p h, 0} \eta_{i} A_{\text {detector }}
$$

where $\alpha$ is the absorption coefficient in units of inverse length, $\mathrm{x}$ is the distance into the semiconductor material, $\mathrm{I}_{\mathrm{ph}, 0}$ is the incident photon irradiance (photons $/ \mathrm{cm}^{2} \mathrm{~s}$ ), $\eta_{i}$ is the internal quantum efficiency defined as the ratio of the current-contributing carriers to the total number of generated carriers, and $\mathrm{A}_{\text {detector }}$ is the effective area of the detector [44]. The quantity of interest is the incident photon irradiance $\mathrm{I}_{\mathrm{ph}, 0}$ which is related to the optical intensity $\mathrm{I}_{0}$ with units of $\mathrm{W} / \mathrm{cm}^{2}$ by equation 3.34 ,

$$
I_{p h, 0}=\frac{I_{0}}{h v}
$$

where $h v$ is the photon energy $[2,44]$. The incident optical intensity $\mathrm{I}_{0}$ is related to the square of the incident electric field $\mathbf{E}_{\mathbf{0}}$ by equation 3.35,

$$
I_{0}=\frac{1}{2} c \varepsilon_{d} \varepsilon_{0}|\boldsymbol{E}|^{2}
$$

where $\mathrm{c}$ is the speed of light $\left(2.998 \times 10^{8} \mathrm{~m} / \mathrm{s}\right)$, and $\varepsilon_{0}$ is the permittivity of free space $\left(8.854 \times 10^{-12} \mathrm{~F} / \mathrm{m}\right)[1-3]$. If the intensity of the incident light is related to the electric field strength, then through the excitation of surface plasmons the electric field strength is increased by a factor of $\left|\frac{E_{S P}}{E_{0}}\right|$ at resonance. The square of this electric field enhancement can then be used to derive the excess carrier generation rate $G_{L_{-} S P}$ in surface plasmon enhanced photoconductive detectors by equation 3.36 . 


$$
\mathrm{G}_{\mathrm{L}, \mathrm{SP}}=\frac{\left(1-\mathrm{e}^{-\alpha \mathrm{x}}\right) \varepsilon_{0} \varepsilon_{\mathrm{d}}\left(\left|\frac{\mathrm{E}_{\mathbf{S P}}}{\mathbf{E}_{\mathbf{0}}}\right|\right)^{2}\left|\mathbf{E}_{\mathbf{0}}\right|^{2} \eta_{\mathrm{i}} \mathrm{A}_{\text {detector }}}{2 \mathrm{~h} \nu}=\left|\frac{\mathrm{E}_{\mathbf{S P}}}{\mathbf{E}_{\mathbf{0}}}\right|^{2} \mathrm{G}_{\mathrm{L}}
$$

The importance of equation 3.32 can be seen by examining equations 3.36 , and observing that the square of the electric field enhancement at resonance acts as an effective gain coefficient for the number of generated excess carriers. For instance, an electric field enhancement of 10 at the surface of the metal sphere would result in a 100x increase in the carrier generation rate, while a field enhancement of 20 would result in a factor of 400 more carriers being generated in the same amount of time over the dielectric host material alone. To the best of the author's knowledge as of the time of this writing, the author was the first to explicitly derive and present this relationship [45]. It should be noted that this relationship was one of the theoretical motivations for investigating surface plasmon resonance for enhanced sensitivity in PbSe photoconductors.

Recall that the major interactions that can occur when light is incident on the metallic particle are reflection, scattering, and absorption. In the quasi-static limit, we ignore the contribution from back-scattering, and only deal with forward-scattering and absorption. In this case, the incident plane wave will be partially transmitted without deviation, partially absorbed, and partially scattered.

The first term to be considered is the absorption cross-section $\sigma_{a b s}$, which is defined as the total power absorbed by the particle, normalized to the intensity of the incident plane wave. Recall that the imaginary part of equation 3.28 represents absorption in the metal. Therefore the imaginary part of the complex polarizability $\alpha^{\prime \prime}$ is also 
representative of absorption, leading to the relationship for the absorption cross-section shown in equation 3.37 ,

$$
\sigma_{a b s}=\frac{k \alpha^{\prime \prime}}{\varepsilon_{0}} \cong \frac{\left(12 \pi k r^{3}\right) \varepsilon_{d} \varepsilon_{m}^{\prime \prime}}{\left(2 \varepsilon_{d}+\varepsilon_{m}^{\prime}\right)^{2}}
$$

where $k=\sqrt{\varepsilon_{d}} k_{0}=\frac{\sqrt{\varepsilon_{d}} 2 \pi}{\lambda_{0}}$ is the wavenumber of the incident plane wave in the surrounding medium in units of inverse length, $\mathrm{r}$ represents the radius of the sphere in $\mathrm{cm}$, and the units of $\sigma_{a b s}$ are $\mathrm{cm}^{2}$. The scattering cross-section $\sigma_{s c a}$ is similarly defined, with the total scattered power being normalized to the intensity of the incident plane wave, in equation 3.38 .

$$
\sigma_{\text {sca }}=\frac{\mathrm{k}^{4}|\alpha|^{2}}{6 \varepsilon_{0}^{2}} \cong \frac{8 \mathrm{k}^{4} \mathrm{r}^{6}}{3}\left|\frac{\varepsilon_{\mathrm{m}}-\varepsilon_{\mathrm{d}}}{\varepsilon_{\mathrm{m}}+2 \varepsilon_{\mathrm{d}}}\right|^{2}
$$

The sum of the scattering and absorption cross-sections, shown in equation 3.39 , is called the extinction cross-section, which represents the total power coupled to a surface plasmon modes normalized to the intensity of the incident plane wave.

$$
\sigma_{e x t}=\sigma_{a b s}+\sigma_{s c a}
$$

Of note are the units of $\sigma_{a b s}, \sigma_{s c a}$, and $\sigma_{\text {ext }}$, which are that area. An intuitive evaluation of the benefits offered by SPPs can be made by examining what is known as the efficiency or Q-factor. The Q-factor is the ratio of a given cross-section to the geometrical cross-section $\mathrm{G}$ of the particle. For spherical particles, the geometrical crosssection is the area of a circle, so the Q-factors associated with surface plasmon resonance are given by equations 3.40-3.42.

$$
Q_{a b s}=\frac{\sigma_{a b s}}{G}=\frac{\sigma_{a b s}}{\pi r^{2}}
$$




$$
\begin{aligned}
& Q_{s c a}=\frac{\sigma_{s c a}}{G}=\frac{\sigma_{s c a}}{\pi r^{2}} \\
& Q_{e x t}=\frac{\sigma_{e x t}}{G}=\frac{\sigma_{e x t}}{\pi r^{2}}
\end{aligned}
$$

Equations 3.40 - 3.42 represent an effective gain factor over the dielectric without metallic nanoparticles. This gain factor will be one of the primary metrics for analytically evaluating the plasmonic response for different metals in $\mathrm{PbSe}$.

\subsubsection{Ellipsoids}

It is important to also discuss ellipsoidal particles in the quasi-static limit, because the particle geometry and orientation with respect to the incident light plays a significant role in determining the resonant frequency [4-10, 12-16]. Ellipsoids are defined mathematically by equation 3.43 ,

$$
\frac{x^{2}}{A^{2}}+\frac{y^{2}}{B^{2}}+\frac{z^{2}}{C^{2}}=1
$$

where $\mathrm{A}, \mathrm{B}$, and $\mathrm{C}$ are the lengths in their respective directions, and $\mathrm{x}, \mathrm{y}$, and $\mathrm{z}$ are defined in equations 3.44-3.46.

$$
\begin{gathered}
x=A \cos \theta \sin \phi \\
y=B \sin \theta \cos \phi \\
z=C \cos \phi
\end{gathered}
$$

Here, $\theta$ and $\phi$ are are contained within the intervals $[0,2 \pi]$ and $[0, \pi]$, respectively [17]. In the special case of ellipsoids in which two of the axes are equal, the shape is called a spheroid. Spheroids are of particular interest, because their shape factors S, described shortly, can be found analytically $[4-5,18-19,22-23,30,45]$. 
There are two cases of spheroids to be considered. The first is the prolate spheroid, in which the relationship between the radii can be defined as in equation 3.47.

$$
C>A=B
$$

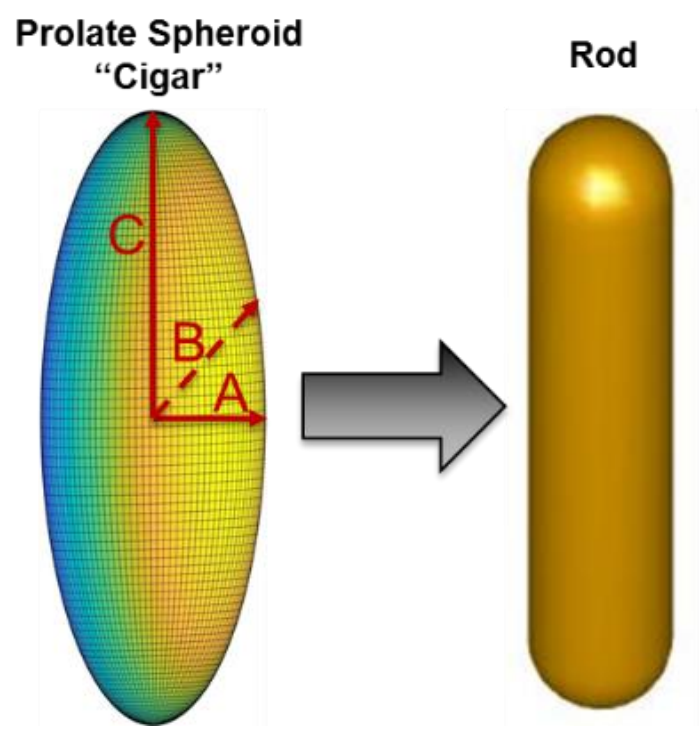

Figure 29. Depiction of a prolate spheroid, which is an analogue of the rod structure.

Figure 29 shows a graphical representation of the prolate spheroid. As the radii of the two ends start to match the radii of $\mathrm{A}$ and $\mathrm{B}$, the shape becomes a rod. Conversely, as the radii of the two ends become smaller and smaller, the prolate spheroid takes on a cigar-like shape. Therefore, prolate spheroid is an analogue for a nanorod, which has many interesting plasmonic properties [18-19, 22-23].

The second spheroidal shape of interest is the oblate spheroid. For the oblate spheroid, the radii follow the relationship as given in equation 3.48.

$$
A=B>C
$$


These structures have a pancake-like shape as the dimension $\mathrm{C}$ becomes smaller. When $\mathrm{C}$ becomes larger, the shape is that of a disc making oblate spheroids acceptable analogues for discs. A graphical representation of the oblate spheroid is shown in figure 33.
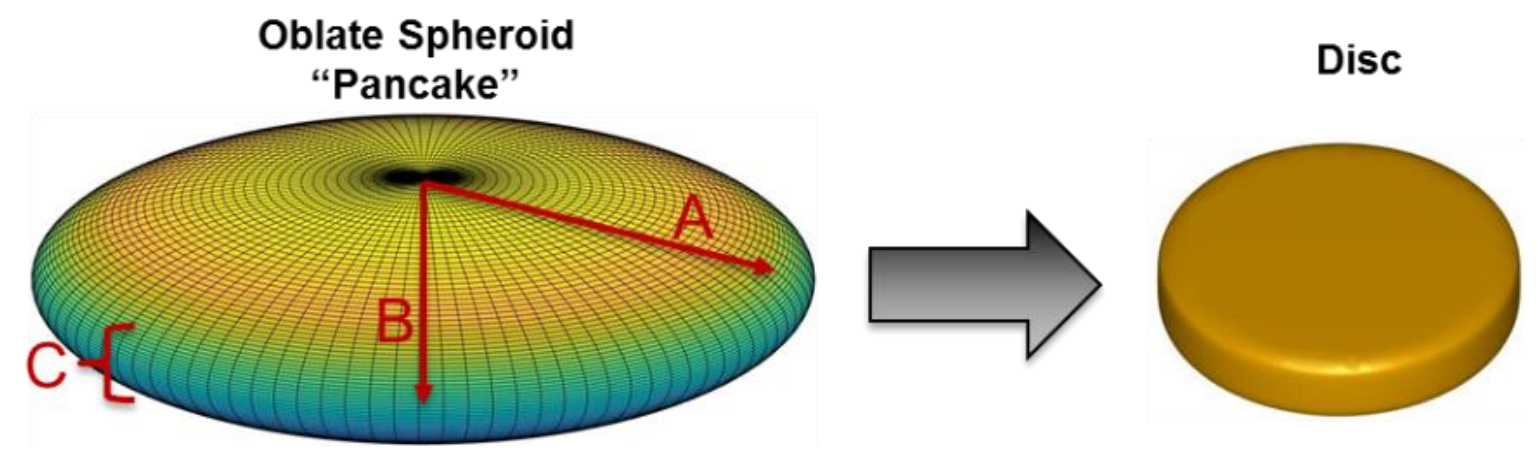

Figure 30. Depiction of an oblate spheroid, which is an analogue of the disc structure.

Returning to the discussion on surface plasmon resonance, the polarizability for ellipsoids is now a tensor quantity dependent on orientation, size, and shape. For the $\mathrm{x}, \mathrm{y}$, and $\mathrm{z}$ directions, the polarizability is given by equations $3.49-3.51$,

$$
\begin{aligned}
& \alpha_{x}=\frac{\left(4 \pi \varepsilon_{0} \varepsilon_{d}\right) A B C\left(\varepsilon_{m}-\varepsilon_{d}\right)}{3\left[\varepsilon_{d}+S_{x}\left(\varepsilon_{m}-\varepsilon_{d}\right)\right]} \\
& \alpha_{y}=\frac{\left(4 \pi \varepsilon_{0} \varepsilon_{d}\right) A B C\left(\varepsilon_{m}-\varepsilon_{d}\right)}{3\left[\varepsilon_{d}+S_{y}\left(\varepsilon_{m}-\varepsilon_{d}\right)\right]} \\
& \alpha_{\mathrm{z}}=\frac{\left(4 \pi \varepsilon_{0} \varepsilon_{\mathrm{d}}\right) \operatorname{ABC}\left(\varepsilon_{\mathrm{m}}-\varepsilon_{\mathrm{d}}\right)}{3\left[\varepsilon_{\mathrm{d}}+\mathrm{S}_{\mathrm{z}}\left(\varepsilon_{\mathrm{m}}-\varepsilon_{\mathrm{d}}\right)\right]}
\end{aligned}
$$

where $S_{x, y, z}$ are the shape factors in the $x_{-}^{-}, y_{-}$, and $\mathrm{z}$-directions given by equations 3.523.54 .

$$
\begin{aligned}
& S_{x}=\frac{A B C}{2} \int_{0}^{\infty} \frac{d s}{\left(s+A^{2}\right)^{\frac{3}{2}}+\left(s+B^{2}\right)^{\frac{1}{2}}+\left(s+C^{2}\right)^{\frac{1}{2}}} \\
& S_{y}=\frac{A B C}{2} \int_{0}^{\infty} \frac{d s}{\left(s+A^{2}\right)^{\frac{1}{2}}+\left(s+B^{2}\right)^{\frac{3}{2}}+\left(s+C^{2}\right)^{\frac{1}{2}}}
\end{aligned}
$$




$$
\mathrm{S}_{\mathrm{z}}=\frac{\mathrm{ABC}}{2} \int_{0}^{\infty} \frac{\mathrm{ds}}{\left(\mathrm{s}+\mathrm{A}^{2}\right)^{\frac{1}{2}}+\left(\mathrm{s}+\mathrm{B}^{2}\right)^{\frac{1}{2}}+\left(\mathrm{s}+\mathrm{C}^{2}\right)^{\frac{3}{2}}}
$$

Equation 3.55 provides a convenient relationship between the shape factors, making analytical calculations of spheroids possible.

$$
S_{x}+S_{y}+S_{z}=1
$$

Consider an X-polarized plane wave incident on a prolate-spheroid. Then the shape factor in the $\mathrm{x}$-direction reduces to the equation 3.56 ,

$$
\begin{gathered}
S_{x}=\left(\frac{1}{1-r_{a s p}^{2}}\right)-\frac{r_{a s p}}{\left(1-r_{a s p}^{2}\right)^{\frac{3}{2}}} \sin ^{-1} \sqrt{1-r_{a s p}^{2}} \\
=\frac{\left(1-e^{2}\right)}{e^{2}}\left[\frac{1}{2 e} \ln \left(\frac{1+e}{1-e}\right)-1\right]
\end{gathered}
$$

where $\mathrm{r}_{\text {asp }}$ is the aspect ratio given as the ratio of the $\mathrm{x}$-dimension to that of the $\mathrm{y}$ - or $\mathrm{z}$ dimensions, and $\mathrm{e}$ is the eccentricity given as $1-r_{a s p}^{-2}$. Now, the $\mathrm{y}$ - and $\mathrm{z}$-shape factors can be found by utilizing the relationship in equation in equation 3.55 , the results of which are shown in equation 3.57 .

$$
S_{y}=S_{z}=\frac{1-S_{x}}{2}
$$

Recall that the resonant plasmonic frequency for spheres in the quasi-static limit is only dependent on the properties of the metal comprising the sphere and the host material. The resonance condition for ellipsoids, however, includes the shape factors and is given by equation 3.58 ,

$$
\varepsilon_{m}^{\prime} \cong \varepsilon_{d}\left(1-\frac{1}{s_{i}}\right)
$$


where the subscript $i$ on the shape factor denotes the direction under consideration. There are several key observations that can be made from equation 3.58. First, in addition to the properties of the metal and host material, the resonance condition now depends on the shape factor. So, depending on the morphology of an ellipsoidal particle, the resonance can be tuned to longer wavelengths [7-18-19]. Further, there will be three different resonance conditions corresponding to the different directions. Therefore, the resonance will be dependent on shape and orientation of the spheroidal structures, as well as the polarization of incident light. It should be noted that if the $\mathrm{x}-, \mathrm{y}-$, and $\mathrm{z}$-axes are equal, equation 3.58 reduces to equation 3.30 .

To gain an intuitive understanding for how the shape of a particle affects the resonance condition, we continue the discussion of prolate-spheroids. As the aspect ratio increases, the surface charges at either end of the particle become more separated $[4,18$ 19]. If the incident plane wave is polarized along the major axis of the spheroid, then the shape factor decreases and the energy of the resonant mode decreases red-shifting the resonant wavelength. In other words, the surface charges at either side of the particle are repulsive, and pushing them further apart decreases the energy in the particle-system [4]. The SP resonance will now occur for wavelengths in which $\varepsilon_{m}{ }^{\prime}$ is more negative. In general, lengthening a particle will cause the resonance along the lengthened direction to shift to longer wavelengths [7-8]. Another phenomenon, known as the "lightning rod" effect, which describes the increase in the local field strength in a rod-like particle, also occurs at the ends of the particle [4, 18-19]. The field strength is inversely proportional to length, so in the limiting case in which the ends of a rod-like particle become like zero- 
dimensional needle points the field strength would become infinite. Of course, for a real particle, the ends would have some finite dimension.

Another important system is the dual- or multi-particle system [4, 14, 20-21]. Similar to the ellipsoidal particle, the resonance condition is polarization and orientation dependent. For simplicity, we consider a dual-particle system of discs positioned adjacent to each other with a small gap between them. If these discs are illuminated by a plane wave polarized along the axis between the discs, their surface charges become attractive [20-21]. Moving the particles closer together would then reduce the energy in the system, and thus shift the resonance to longer wavelengths. Conversely, if the plane wave is polarized perpendicular to the axis between the particles, then the energy in the system increases and the resonant wavelength is blue shifted $[4,20]$. In either case, due to the small gap, a very large electric field will accumulate between the particles [22]. This phenomenon is called the dual-dipole effect [4, 20-22].

\subsubsection{Plasmonics in the Mie Regime}

The quasi-static or Rayleigh regime is a subset of Mie theory. The upper limit of the classical regime typically occurs when the particle size is between $1 \%$ [4] and $10 \%$ [7] of the wavelength. When the particle or structure size approaches the wavelength of light, then Mie theory must be used. In the Mie regime, resonances are dependent on morphology and size, where in general the resonance redshifts for larger particles [1213]. Further, the relaxation time of plasmons in the metal must now be considered [4]. As the relaxation time decreases, the resonances become broadened [4-5, 23-24]. Moreover, where we only considered the dipole resonance in the quasi-static limit, we must now consider multi-pole resonances [4-5, 12-13]. For a detailed derivation of Mie theory, see 
[12-13]. For now, the scattering, extinction, and absorption cross-sections will be given without derivation. Assuming plane wave illumination, the scattering, extinction, and absorption cross-sections in Mie theory are given by equations 3.59-3.61,

$$
\begin{gathered}
\sigma_{s c a}=\frac{2 \pi}{k_{m}^{2}} \sum_{l=1}^{\infty}(2 l+1)\left(\left|a_{l}\right|^{2}+\left|b_{l}\right|^{2}\right) \\
\sigma_{e x t}=\frac{2 \pi}{k_{m}^{2}} \operatorname{Re}\left\{\sum_{l=1}^{\infty}(2 l+1)\left(a_{l}+b_{l}\right)\right\} \\
\sigma_{a b s}=\sigma_{e x t}-\sigma_{s c a}
\end{gathered}
$$

where 1 indicates the multi-pole resonant mode (for a dipole, $1=1$ ), al and $b_{1}$ are the Mie coefficients representing scattering, and the wavenumber $\mathrm{k}_{\mathrm{m}}$ is related to the size parameter $\chi$ by equation 3.62 [4],

$$
\chi=k_{m} r
$$

where for a sphere $r$ represents the radius. There are in fact four Mie coefficients that represent scattering $\left(a_{1}, b_{1}\right)$, backscattering $\left(c_{1}\right)$, and absorption $\left(d_{1}\right)$ [13]. The coefficients $c_{1}$ and $d_{1}$ represent the internal fields of the particle, while $a_{1}$ and $b_{1}$ coefficients coincide with the external/scattered fields of the particle. Modifying equations $3.40-3.42$ to account for the new size parameter $\chi$, the Q-factors for scattering, absorption, and extinction can be found.

\subsection{Investigating the Plasmonic Response in PbSe}

Shown in Chapter 2, PbSe has a bandgap of $0.29 \mathrm{eV}$. For this investigation, the detector films are sensitized with iodine, forming a top layer of $\mathrm{PbI}_{2}$ which has a bandgap of $\sim 2.4 \mathrm{eV}$. The band of interest for these detector materials is in the mid-infrared (3-5 $\mu \mathrm{m})$, so from equation 1.4 in Chapter 1 it is clear that light incident on the detector films should pass through the $\mathrm{PbI}_{2}$ and be absorbed by the $\mathrm{PbSe}$. Keeping this in mind, 
assuming uniform plane wave illumination, oblate spheroids have been modeled in $\mathrm{PbSe}$ using MATLAB scripts I developed, which can be found in appendix B. Stated in Chapter 1, the plasmonic disc was selected as the structure of choice due to its ease of fabrication, demonstrated sensor enhancement in the literature, wavelength selectivity, and large near-field intensity increase [25-26]. Using the Sellmeier equation, the index of refraction $\mathrm{n}$ for $\mathrm{PbSe}$ in the 3-5 $\mu \mathrm{m}$ range is given by equation 3.63 [27].

$$
n^{2}-1=\frac{21.1 \lambda^{2}}{\lambda^{2}-1.37^{2}}
$$

For $3 \mu \mathrm{m}$, the index of refraction of PbSe was calculated to be 5.27, and for $5 \mu \mathrm{m}$ the index of refraction was found to be 4.88. For the following calculations a uniform index over the spectral band of interest was assumed, so the calculated average refractive index of 5.07 was used over the $3 \mu \mathrm{m}$ and $5 \mu \mathrm{m}$ band. The Sellmeier equation, as opposed to the full dispersion relations, was used for simplicity as a first approximation for numerical calculations. It should be noted that the following results include only the resonance conditions and Q-factors in PbSe. Later, the effect of nanostructures on the absorption of $\mathrm{PbSe}$ will be treated in a more rigorous fashion using the finite element analysis method.

\subsubsection{Dispersion Relations of Drude Metals}

To determine a suitable metal for surface plasmon resonance in $\mathrm{PbSe}$, various Drude metals were investigated. Using the compiled and tabulated constants for the Lorentz-Drude model from Rakic, the dispersion relations have been found for Ag, Al, $\mathrm{Au}, \mathrm{Ni}, \mathrm{Pd}$, and $\mathrm{Pt}$ [28]. The complex permittivity was determined using equation 3.64 and 3.65, where the former is the single oscillator permittivity, and the latter accounts for many oscillators within the metal. 


$$
\begin{gathered}
\varepsilon_{r 0}(\omega)=1-\frac{\Omega_{p}^{2}}{\omega^{2}-i \omega \Gamma_{0}} \\
\varepsilon_{r}(\omega)=\varepsilon_{r 0}+\sum_{i=1}^{m} \frac{f_{i} \omega_{p}^{2}}{\left(\omega_{i}^{2}-\omega^{2}\right)+i \omega \Gamma_{i}}
\end{gathered}
$$

Here, $\Omega_{p}=\sqrt{f_{0}} \omega_{p}$ is the intraband transition, also known as the free-electron, plasma frequency, $\mathrm{f}$ is the oscillator strength which is an indication of the probability of absorption in the transition between the energy levels of individual oscillators, $\Gamma$ is the damping constant, $\omega_{i}$ is the oscillator frequency, $\omega_{p}$ is the bulk, also known as the bound-electron, plasma frequency, and $\omega$ is the probe frequency. Specific oscillators are denoted by $i$, where Rakic has divided the calculation between the bulk and individual oscillators within the metal [28]. To compare and evaluate all variables without difficulty, they are provided in units of energy $(\mathrm{eV})$. The complex index was found by taking the square root of equation 3.65. The MATLAB scripts the author developed for the calculation of the dispersion relations of these metals can be found in Appendix B.

The properties of plasmonic structures, as well as their host material, not only govern the resonance, but also govern the surface plasmon dynamics [4, 32]. Important surface plasmon properties include the propagation distance $\mathrm{D}_{\mathrm{SP}}$, the phase velocity $\mathrm{V}_{\mathrm{SP}}$, and the surface plasmon lifetime $\tau_{S P}$ [4]. The propagation distance, given in equation 3.66, for an ideal metal is a measure of the length over which the metal can support the propagation of surface plasmons before their amplitude decays to 1/e of their initial value.

$$
D_{S P} \cong \frac{2 \varepsilon_{m}^{\prime 2}}{\left(\varepsilon_{d}\right)^{\frac{3}{2}} \varepsilon_{m}^{\prime \prime} k_{0}}
$$

The surface plasmon phase velocity is given in equation 3.67 , which is needed to find the surface plasmon lifetime by equation 3.68, 


$$
\begin{gathered}
V_{S P}=\frac{\omega}{k_{S P}}=c \sqrt{\frac{\varepsilon_{d}+\varepsilon_{m}}{\varepsilon_{d} \varepsilon_{m}}} \\
\tau_{S P}=\frac{D_{S P}}{V_{S P}} \cong \frac{2\left(\varepsilon_{m}^{\prime}\right)^{2}}{\omega \varepsilon_{d} \varepsilon_{m}^{\prime \prime}}
\end{gathered}
$$

where $\omega$ is the angular frequency of the incident light wave, $\mathrm{k}$ is the wavenumber, $\varepsilon_{d}$ is the relative dielectric constant of the host material, and $\varepsilon_{m}{ }^{\prime}$ and $\varepsilon_{m}^{\prime \prime}$ are the real and imaginary parts of the relative permittivity of the metal, respectively. Stated above, the surface plasmon lifetime is an important parameter that is inversely proportional to the width of resonant peaks, defined as the full-width-at-half-maximum (FWHM). Surface plamons can be limited by decay mechanisms such as nonradiative decay due to electronhole pairs generated in the metal [34], as well as a loss of phase coherence due to scattering mechanisms [35]. In general, in real metals with finite surface plasmon lifetimes, a shorter-lived lifetime leads to a broader resonance peak with lower Q-factors and electric field enhancement [36].

To determine the MWIR surface plasmon properties of the metals listed above, I calculated the surface plasmon propagation distance, surface plasmon phase velocity normalized to the speed of light in vacuum, and surface plasmon lifetime. The results of this calculation were used to evaluate which metal was best suited for surface plasmon enhanced PbSe detectors. The host material was assumed to be $\mathrm{PbSe}$ with a refractive index of 5.07.

Table 5 shows the calculated results at the cutoff wavelength $(4.3 \mu \mathrm{m})$ of $\mathrm{PbSe}$, and figure 31 - figure 33 show the trend from $2.5 \mu \mathrm{m}-6 \mu \mathrm{m}$. We find that $\mathrm{Ag}, \mathrm{Al}$, and Au have the highest surface plasmon characteristics, with the calculated propagation distance reaching as high as $\sim 50 \mu \mathrm{m}$ and the surface plasmon lifetime being as high as 
800 ns. In contrast, the calculated characteristics for $\mathrm{Ni}, \mathrm{Pd}$, and Pt showed a factor of 5to 10-times lower propagation distance and lifetime compared to that of $\mathrm{Ag}, \mathrm{Al}$, and $\mathrm{Au}$. These results suggest the metals in the top part of table 5 will yield sharper resonances with higher field-enchantment than those in the bottom half of the table.

Table 5. Calculated surface plasmon characteristics for various metals embedded in PbSe.

\begin{tabular}{|c|c|c|c|}
\hline Metal & $\begin{array}{c}\text { DsP at 4.3 } \boldsymbol{\mu m} \\
(\boldsymbol{\mu m})\end{array}$ & VsP/c at 4.3 $\boldsymbol{\mu m}$ & $\begin{array}{c}\boldsymbol{\tau}_{\text {SP }} \text { at } \mathbf{4 . 3} \boldsymbol{\mu m} \\
(\mathbf{n s})\end{array}$ \\
\hline $\mathrm{Ag}$ & 48.4 & 0.200 & 818 \\
\hline $\mathrm{Al}$ & 42.7 & 0.199 & 722 \\
\hline $\mathrm{Au}$ & 35.6 & 0.201 & 602 \\
\hline $\mathrm{Ni}$ & 4.13 & 0.208 & 697 \\
\hline $\mathrm{Pd}$ & 8.27 & 0.205 & 101 \\
\hline $\mathrm{Pt}$ & 6.48 & 0.207 & 110 \\
\hline
\end{tabular}

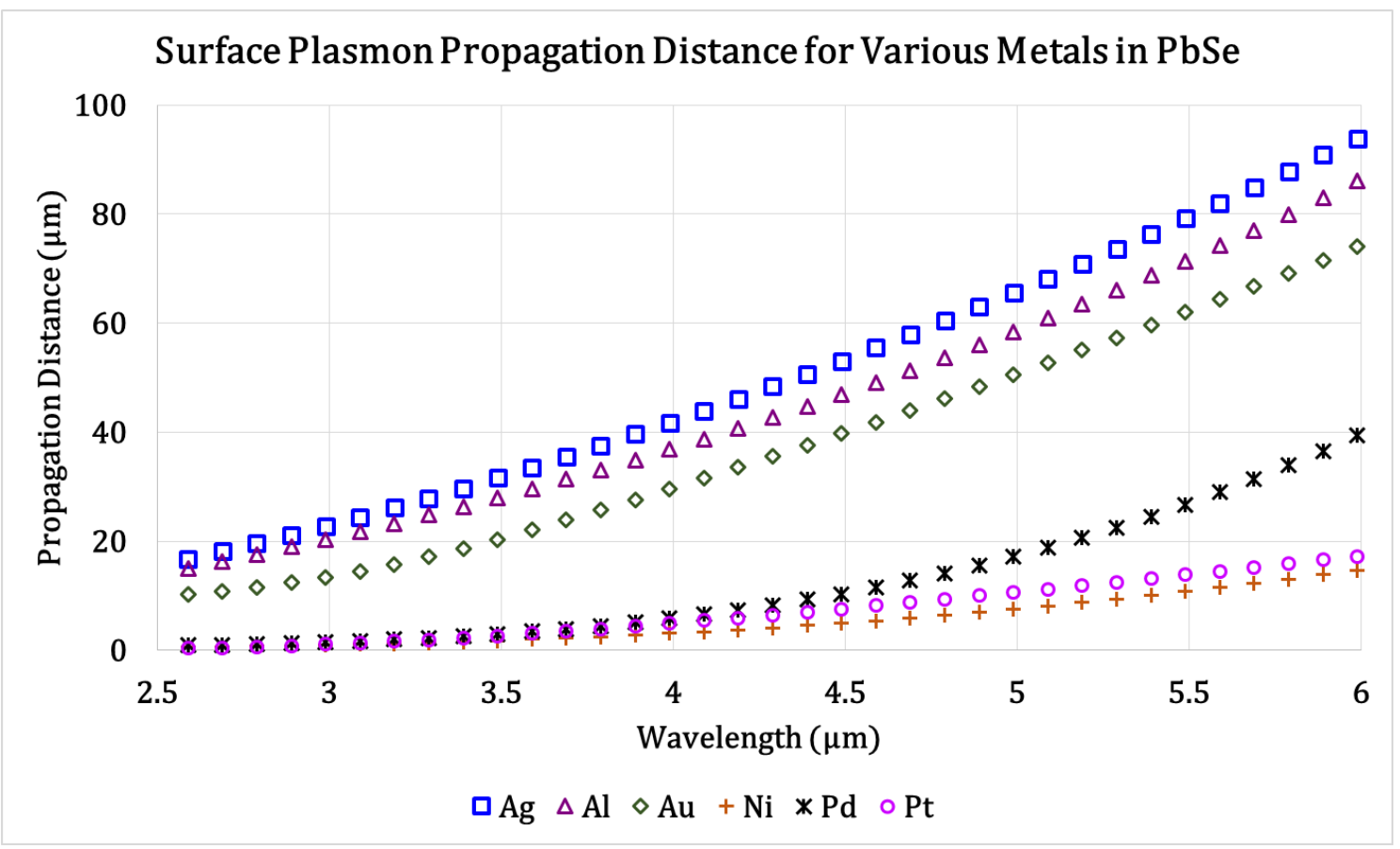

Figure 31. Surface plasmon propagation distance for various metals embedded in PbSe. 
The observed trends from the calculated surface plasmon characteristics, shown in figure 31 - figure 33, show that the surface plasmon propagation distance and lifetime increase with wavelength for all the metals. This is however an idealization, and does not account for surface roughness or the change of the refractive index of $\mathrm{PbSe}$ with wavelength. Moreover, these calculations do not account for morphology or size of the metallic structures, which can have a significant effect on the surface plasmon characteristics [34]. In addition to surface roughness and morphology considerations, the $\mathrm{PbSe}$ deposition and sensitization process must also be considered. Nobel metals like Au have been found in previous experiments to diffuse into our PbSe films during the sensitization process. Therefore, metals like Pt, though their enhancement may not be as great as the nobel metals, were found to offer a feasible alternative for fabricating surface plasmon resonant structures into PbSe detectors.

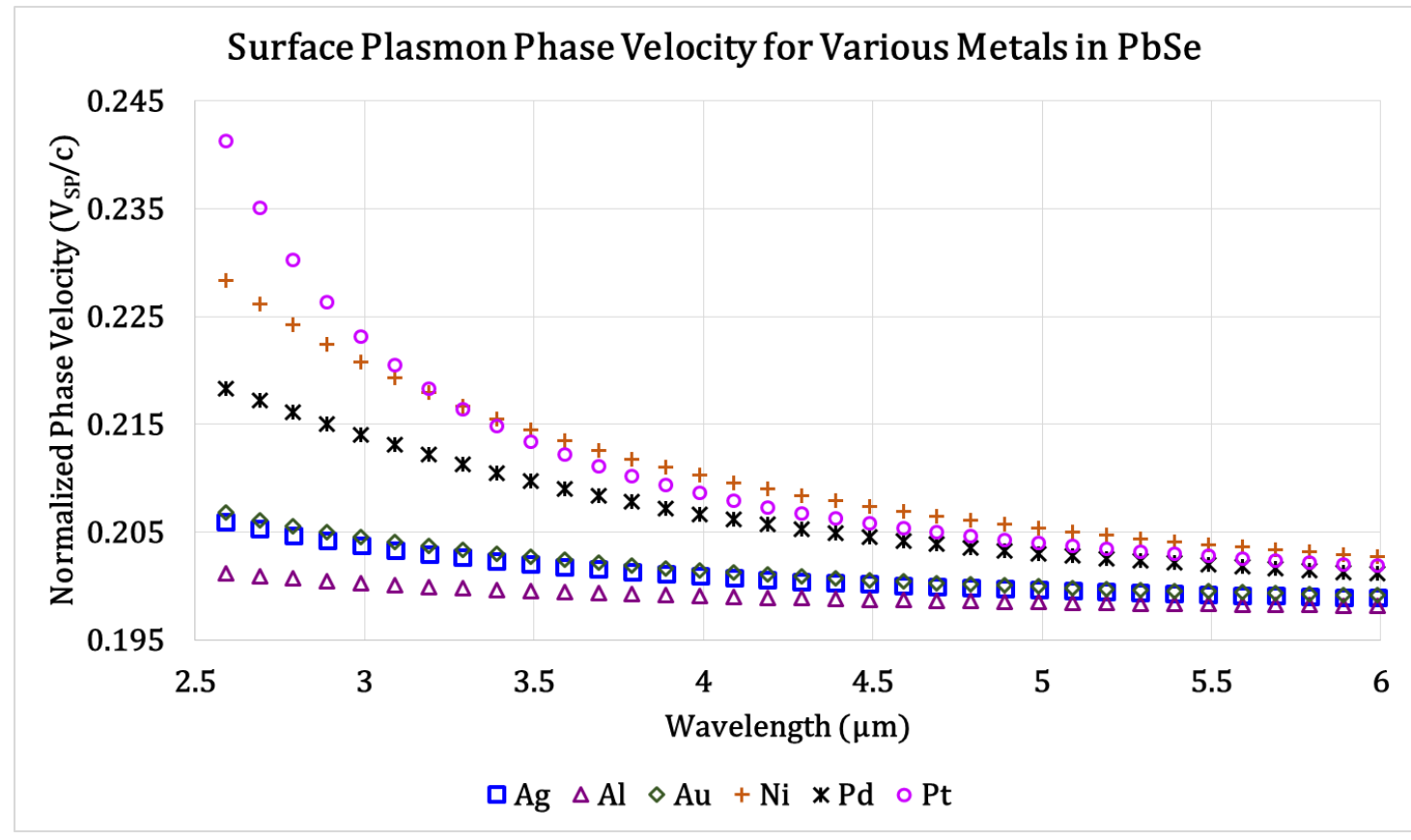

Figure 32. Phase velocity normalized to the speed of light in vacuum of surface plasmons for various metals in PbSe. 
The nanodisc is polarization independent if transversely illuminated from above, assuming the disc is oriented such that its circular face is perpendicular to the propagation vector of the incident light wave [4-5, 25-26]. Assuming the source of the light wave is above the disc and traveling in the $-\mathrm{z}$-direction, the face of the nanodisc is a circle of radius $\mathrm{A}$. The dimension $\mathrm{C}$ is half the thickness of the disc, and the aspect ratio is $r_{a s p}=$ $A / C$. Mentioned above, the nanodisc is relatively simple to fabricate with techniques such as electron beam lithography, photolithography, and nanosphere lithography [21, 30].

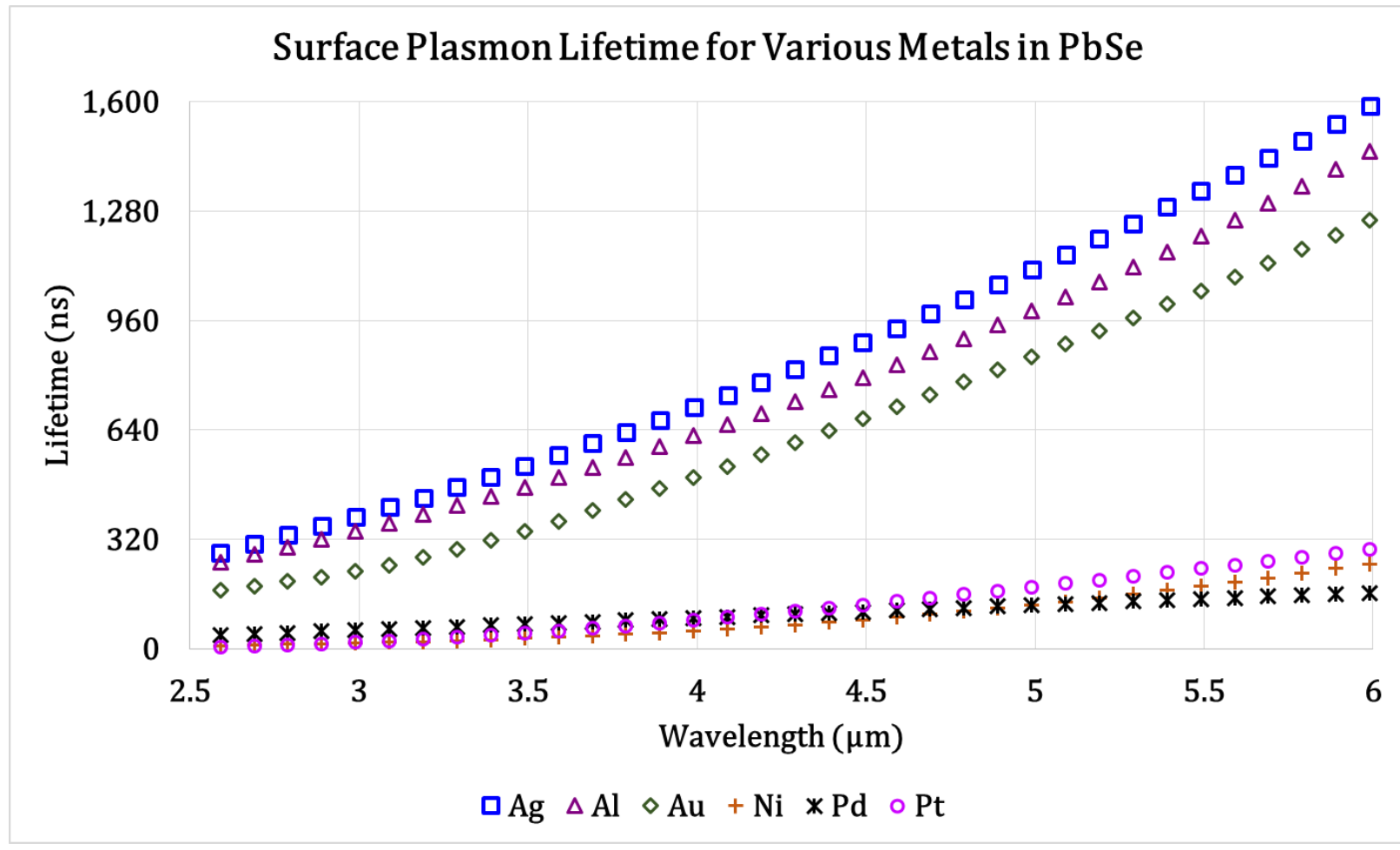

Figure 33. Surface plasmon lifetime for various metals in PbSe.

Due to their polarization independence, relative ease of fabrication, and demonstrated enhancement in similar materials, this investigation was focused on the nanodisc structure. Recall that the $\mathrm{SiO}_{2}$ layer of the films is roughened to a peak-to-peak 
value of $500 \mathrm{~nm}$ to ensure the PbSe adheres to the substrate. Coupled with the inherent roughness of the polycrystalline grains of $\mathrm{PbSe}$, this surface roughness permeates throughout the $\mathrm{PbSe}$ film. Therefore, it would be difficult to adequately fabricate structures within the PbSe layer. The limitation stems from the coherent nature of surface plasmons [4-5, 33, 34-35]. Surface plasmons have a degree of coherence, and the waves of which they are comprised retain a direct phase relationship for short distance within the near-field of the structure surface. The surface roughness of the metal can act as scattering centers, thereby changing the path length of various parts of the traveling evanescent waves reducing their coherence length [31-32]. In fact, if the surface is too rough it may negate any surface plasmon enhancement. Therefore, the nanodiscs in this study will be considered at the $\mathrm{PbSe} / \mathrm{SiO}_{2}$ interface. Practically, discs were fabricated on smooth $\mathrm{SiO}_{2}$, and a newly developed roughening procedure, described in Appendix A, was performed around the discs.

For the investigation of PbSe films, nanodiscs were modeled numerically using the quasi-static derivation described above. For a structure located at an interface between two dielectric materials, the effective index of the host material is the average of the two materials [22, 37-39]. Therefore, the effective index of refraction at the interface between $\mathrm{PbSe}$ and $\mathrm{SiO}_{2}$ over the 3-5 $\mu \mathrm{m}$ band is considered to be 3.24 , because $\mathrm{SiO}_{2}$ has an in-band refractive index of 1.41. Figure 34 shows the comparison of extinction crosssections of nanodiscs with aspect ratios of 10, 13, 15, 18, and 20. As the aspect ratio increases, the resonance peak red-shifts to longer wavelengths as would be expected. It was found in figure 34 that an increasing the aspect ratio of the Pt nanodisc from 10 to 20 lead to a resonance peak that coincides with the PbSe absorption edge at $4.27 \mu \mathrm{m}$. 
Furthermore, the peaks become broader suggesting a decreased surface plasmon lifetime $[4,35]$. Finally, the amplitude of the extinction cross-section increases with aspect ratio due to the increased diameter.

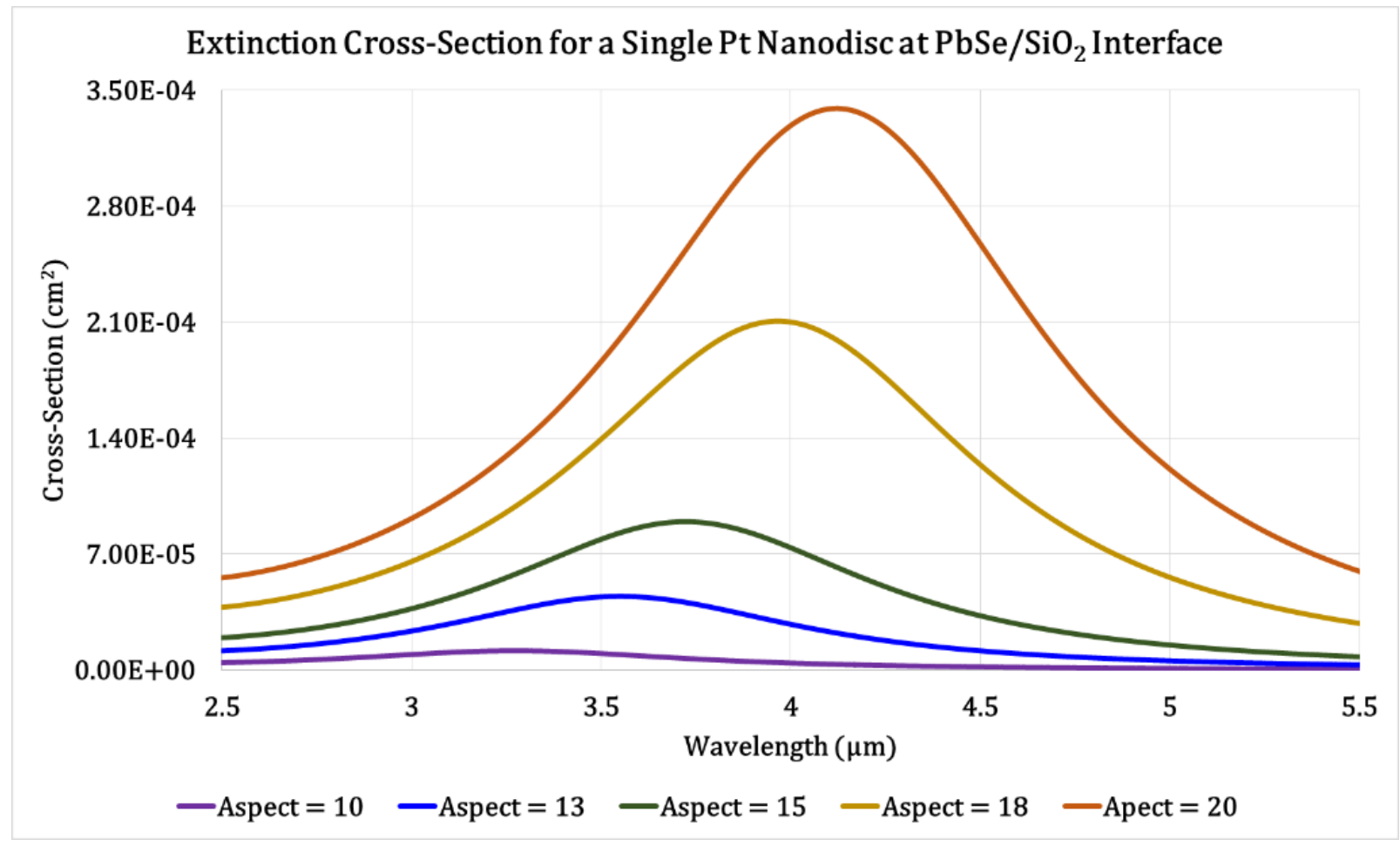

Figure 34. Extinction cross-section of a single Pt nanodisc at the PbSe/SiO 2 interface. Resonance peak redshifts with wavelength, demonstrating wavelength selectivity for designing surface plasmon resonant disc structures for detector enhancement.

For the Pt discs in figure 34 it would seem that an aspect ratio of 20 would be the optimum design choice, because the resonance peak coincides with the absorption edge of $\mathrm{PbSe}$ and has the largest amplitude. However, the PbSe absorption edge also coincides with the atmospheric $\mathrm{CO}_{2}$ absorption notch [33]. If the resonance is slightly blue shifted, then the peak can still be within the PbSe spectral sensitivity band while remaining close the absorption edge. To this end, an aspect ratio between 13 and 18 would be preferred. 
I also calculated the field enhancement of $\mathrm{Pt}$ discs at the $\mathrm{PbSe}_{-} \mathrm{SiO}_{2}$ interface.

Using the MNPBEM MATLAB toolbox, a freely available simulation tool which uses the boundary element method to calculate the solution to Maxwell's equations using surface charges, the near-field intensity enhancement $\frac{|E|^{2}}{\left|E_{0}\right|^{2}}$ was calculated [40-41]. Moreover, this tool was also used to observe the effect of the incident illumination angle on the scattering cross-section and field-enhancement. Modeled discs were $250 \mathrm{~nm}$ in diameter and $20 \mathrm{~nm}$ thick, which resulted in an aspect ratio of 12.5.

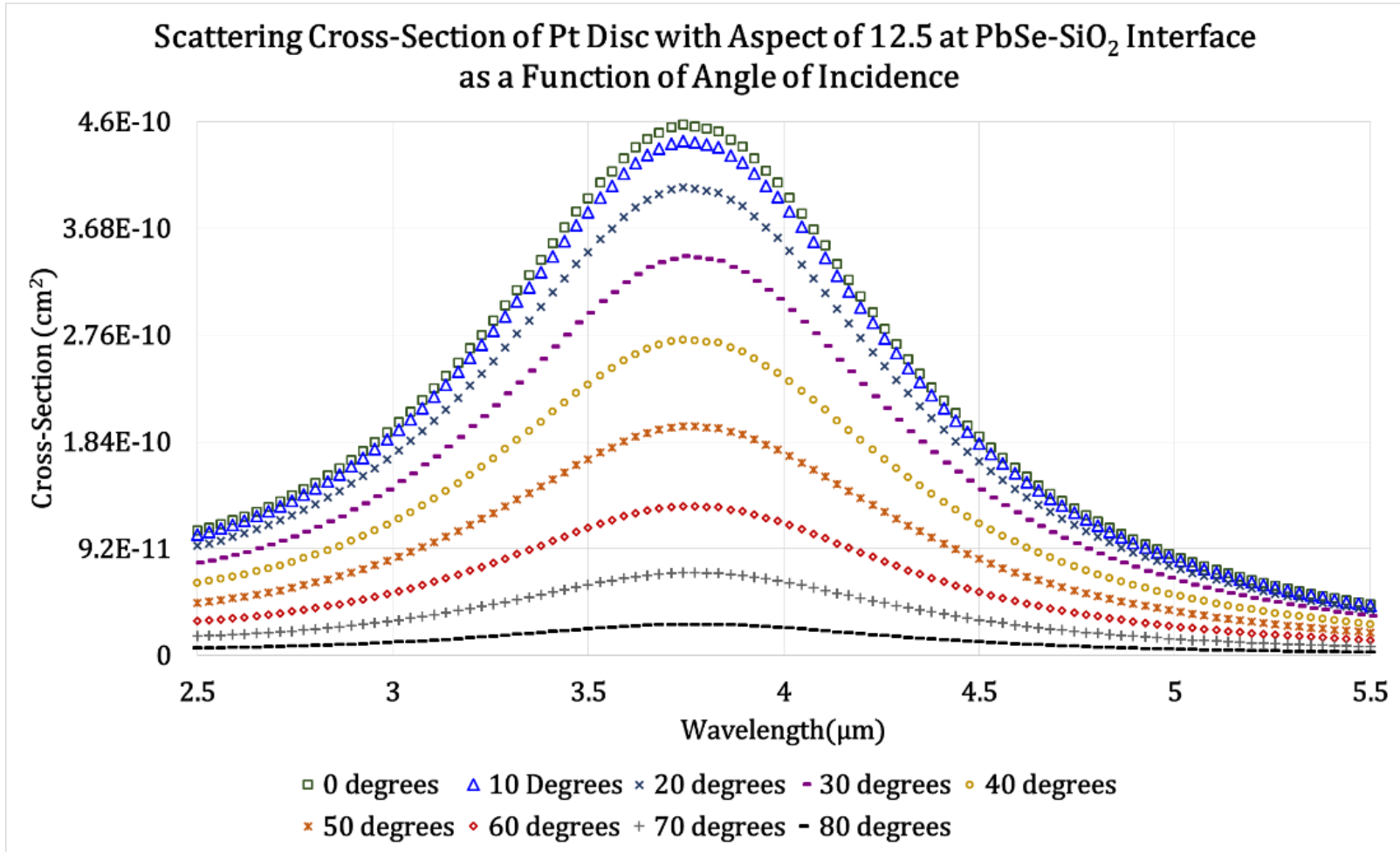

Figure 35. Calculated scattering-cross section for surface plasmon resonant discs at the PbSe-SiO interface as a function of incident illumination angle.

The calculated scattering cross-section as a function of incident illumination angle is shown in figure 35 . For all angles, the resonance was found to be $3.7 \mu \mathrm{m}$, which is slightly red-shifted from the results shown in figure 34 . Further, the amplitudes differ by 
several orders-of-magnitude, with the quasi-static calculation yielding more idealistic results. The discrepancy is due to the different algorithms used for the calculation. In figure 34 the quasi-static limit was used, while in figure 35 the more rigorous boundary element method was used. Nevertheless, the results yield similar resonances. Of note in figure 35 is the fact that the maximum scattering-cross section was found at a normal angle of incidence. This is an important feature of the plasmonic disc structure, because the investigated $\mathrm{PbSe}$ detectors are designed to be illuminated at normal incidence.

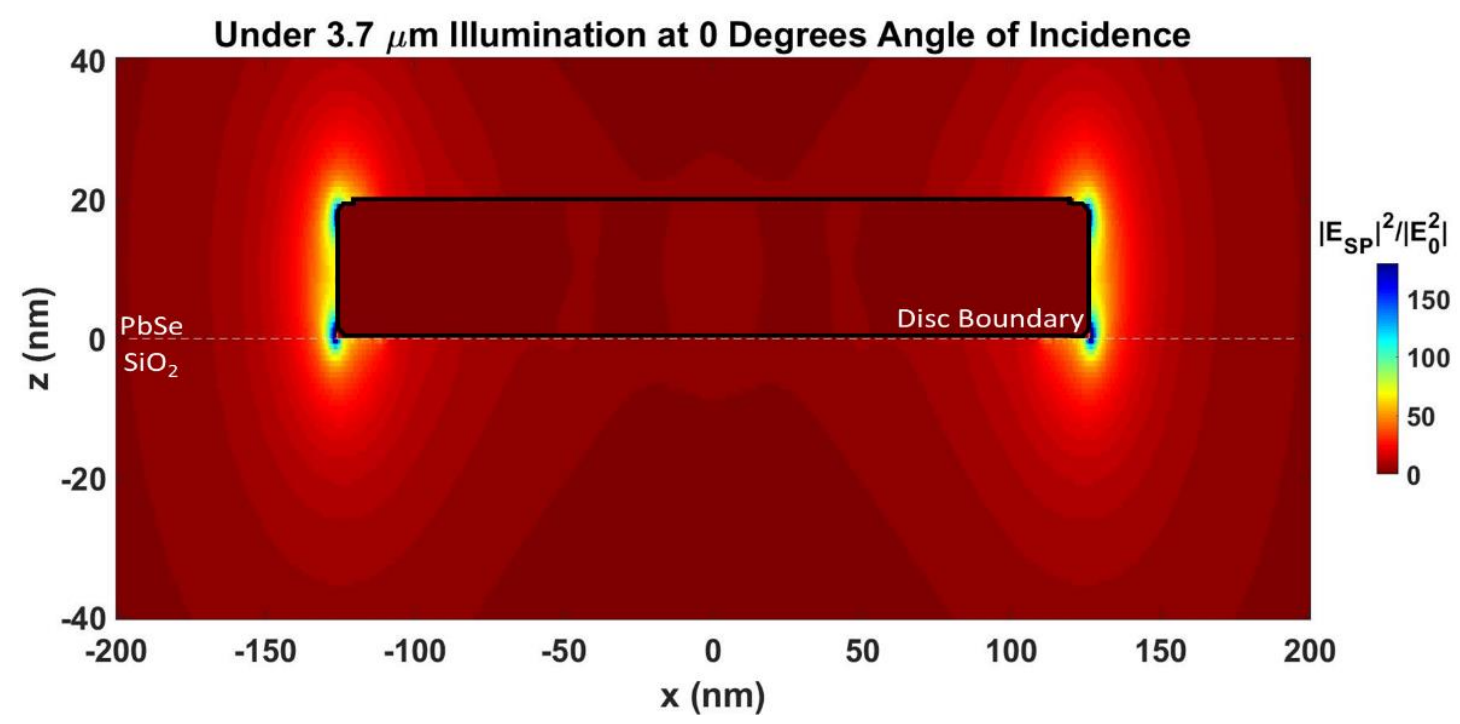

Figure 36. Field intensity enhancement map of a Pt disc located at the $\mathrm{SiO}_{2}-\mathrm{PbSe}$ interface illuminated with a $3.7 \mu \mathrm{m}$ plane wave at normal incidence

The electric field intensity enhancement for a Pt disc illuminated at normal incidence with an excitation wavelength of $3.7 \mu \mathrm{m}$ is shown in figure 36 . The near-field intensity enhancement, which is the square of equation 3.31 modified by the morphology of the disc, is predicted be as high as 200 on the disc surface, and up to 75 a distance of $25 \mathrm{~nm}$ into the PbSe layer. Distances of $\sim 90 \mathrm{~nm}$ into the PbSe layer still experience a near field intensity enhancement greater than that of the incident field. Even on the inside of the 
discs, which shows the induced field intensity indicative of surface plasmon resonance, a small field intensity increase is observed. The field intensity enhancement map shown in figure 36, which was only considered as an ideal approximation, showed that an appreciable increase in absorption and carrier generation in the investigated PbSe films was feasible.

Before moving on from this chapter, there are a couple of important comments to make with respect to figure 34 - figure 36 . The calculated results assumed an aspect ratio of 12.5 , yet the question may arise as to how large the disc diameter can be and still show the same surface plasmon resonance characteristics. Part of the answer comes down to the limitation of the fabrication techniques to be employed. For instance, for larger structures, ultraviolet (UV) photolithography might be chosen for its relatively quick process and ability to cover large areas [42-43]. Yet, if UV photolithography is chosen, there is a limit as to how small the structures can be accurately reproduced in photoresist, which is dependent on the minimum feature size of the mask and the diffraction limit for the wavelength of the UV light. Modeling is a powerful tool for assessing what aspect ratio is required and/or desired for the particular application and spectral band. From there, the limits of the fabrication technique will dictate the actual size of the structure.

The second discussion point, which is closely related to the first, is wavelength selectivity. The spectral band of interest and the bandwidth are dependent on the application for which a detector system will be used. Figure 34 demonstrates the wavelength selectivity offered by the surface plasmon resonant disc structure. Depending on the application, spectral band, and bandwidth of interest, the structure can be designed and tailored. 


\section{Chapter 3 References}

[1] J. D. Jackson, Classical electrodynamics, New York: Wiley, 1999.

[2] J. D. Griffiths, Introduction to electrodynamics, Upper Saddle River, N.J: Prentice Hall, 1999.

[3] C. A. Balanis, Advanced Engineering Electromagnetics, ${ }^{\text {st }}$ Ed., Hoboken, NJ: John Wiley and Sons, Inc. 1989.

[4] D. Sarid and W. Challner, Modern Introduction to Surface Plasmons: Theory, Mathematical Modeling, and Application, New York, NY: Cambridge University Press, 2010.

[5] A. A. Toropov and T. V. Shubina, Plasmonic Effects in Metal-Semiconductor Nanostructures, Great Clarendon, Oxford, United Kingdom: Oxford University Press, 2015.

[6] M. P. Marder, Condensed Matter Physics, 2nd ed., Hoboken, NJ: John Wiley and Sons, Inc., 2010.

[7] Z. Jaks ic', et al. "Red Shifting Approach for Nanoplasmonic Enhancement of Semiconductor Infrared Detectors.” Proc. 28th International Conference on Microelectronics, Serbia, 2012.

[8] A. Battaglia, et al. "Light scattering by Gaussian particles: Rayleigh-ellipsoid approximation,” J. of Quantitative Spectroscopy and Radiative Transfer, 63, 1999.

[9] P. Berini and A. Olivieri, "Plasmonic Optoelectronics on Si." Proc. Of SPIE, Vol. 9163, 2014.

[10] W. A. Murray and W. L. Barnes, "Plasmonic Materials.” Adv. Mater. 19, 2007. 
[11] C. Kittel, Introduction to Solid State Physics, Hoboken, NJ: John Wiley and Sons, Inc. 2005.

[12] Evan, Amato. University of California San Diego, Scripps Institute of Oceanography, Department of Atmospheric and Climate Sciences 251: Radiation The Atmosphere Ch3 Class Notes

[13] C. Matzler, "MATLAB Functions for Mie Scattering and Absorption," Institut fur Angewandte Physik, Research Report No 2002-08, 2002.

[14] P. Strobbia, et al. "Recent advances in plasmonic nanostructures for sensing: a review," Optical Engineering, Vol. 54, No. 10, 2015.

[15] N. Pere-Laperne, et al. "Infrared Plasmonic Detectors." Proc. of SPIE, Vol. 7945, 2011.

[16] https://nanocomposix.com, visited 2/24/2018

[17] W. E. Weisstein, "Spheroid." From MathWorld - A Wolfram Web Resource [online]. Available: http://mathworld.wolfram.com/Spheroid.html [Accessed: Sept. 11, 2018].

[18] E. S. Kooij and B. Poelsema, "Shape and size effects in the optical properties of metallic nanorods," Phys. Chem. Chem. Phys., Vol. 8, 2006.

[19] J. S. Sekhon and S. S. Verma, "Rational Selection of Nanorod Plasmons: Material, Size, and Shape Dependence Mechanism for Optical Sensors," Plasmonics, Vol. 7, 2012.

[20] T. Atay, J. - H. Song, and A. V. Nurmikko, "Strongly Interacting Plasmon Particle Pairs: From Dipole-Dipole Interaction to Conductively Coupled Regime," Nano Lett., Vol. 4, No. 9, 2004. 
[21] Y. Li, Plasmonic Optics Theory and Applications, Tutorial Texts in Optical Engineering, Vol. TT110. SPIE Press. Billingham, WA., 2017.

[22] J. A. Schuller, et al. "Plasmonics for extreme light concentration and manipulation,” Nature Materials, Vol. 9, 2010.

[23] V. Juve, et al. "Size-Dependent Surface Plasmon Resonance Broadening in Nonspherical Nanoparticles: Single Gold Nanorods,” Nano Lett., Vol. 13, No. 5, 2013.

[24] T. J. -Y. Derrien, et al. "Properties of Plasmon Polaritons on lossy metals: Lifetimes, periods, and excitation conditions," J. of Opt., Vol. 18, No. 11, 2016

[25] X. Tang, et al. "Plasmon resonance enhanced colloidal HgSe quantum dot filterless narrowband photodetectors for mid-wave infrared," J. Mater. Chem. C, $5,2017$.

[26] J. Liang, et al. "Modeling of $\mathrm{HgCdTe}$ photoconductive infrared detector with metallic nanostructures.” IEEE, NUSOD, 2014.

[27] Polyansky, M. RefractiveIndex.info. https://refractiveindex.infor/?shelf=main\&book=PbSe\&page=zemel

[28] Rakic', et al. "Optical Properties of Metallic Films for Vertical Cavity Optoelectronic Devices,” Applied Optics, Vol. 37, No. 22, 1998.

[29] A. I. Golovashkin, and G. P. Motulevich, "Optical Properties of Lead in the Visible and Infrared Spectral Ranges,” Sov. Phys. Jet P 26, 1968.

[30] J. Alda and G. Boremann, Infrared Antennas and Resonant Structures, Billingham, WA: SPIE Press, 2017. 
[31] G. A. Farias and A. A. Maradudin, "Effect of surface roughness on the attenuation of surface polaritons on metal films,” Phys. Rev. B 27, 1983.

[32] A. Degiron and D. R. Smith, "Numerical simulations of long-range plasmons," Optics Express, Vol. 14, No. 4, 2006.

[33] M. Falk and A. G. Miller, "Infrared spectrum of carbon dioxide in aqueous solution," Vibrational Spectroscopy, Vol. 4, Iss. 1, 1992.

[34] U. Freibig and M. Vollmer, Optical Properties of Metal Clusters, Berlin: Springer-Verlag, 1995.

[35] M. Perner, et al. "Optically induced damping of the surface plasmon resonance in gold colloids,” Phys. Rev. Lett., Vol. 78, 1997.

[36] T. Klar, et al. "Surface-plasmon resonances in single metallic noanparticles," Phys. Rev. Lett., Vol. 80, 1998.

[37] P. Strobbia, et al. "Recent advances in plasmonic nanostructures for sensing: a review," Optical Engineering, 54(10), 100902, 2015.

[38] H. N. Daghestani and B. W. Day, "Theory and applications of Surface Plasmon Resonance, Resonant Mirror, Resonant Waveguide Grating, and Dual Polarization Interferometry Biosensors," Sensors, 10, 2010.

[39] E. Li and H. Chu, Plasmonic Nanoelectronics and Sensing, United Kingdom: Cambridge Press, 2014.

[40] U. Hohenester and A. Trugler, "MNPBEM-A Matlab toolbox for the simulation of plasmonic nanoparticles," Computer Physics Communications, Vol. 183, Iss. 2, 2012. 
[41] J. Waxenegger and U. Hohenester, "Plasmonics simulations with the MNPBEM toolbox: Consideration of substrates and layer structures," Computer Physics Communications, Vol. 193, 2014.

[42] S. A. Campbell, Fabrication Engineering at the Micro- and Nanoscale, Fourth Edition, Oxford University Press, New York, 2013.

[43] M. J. Madou, Fundamentals of Microfabrication: The Science of Miniaturization, Second Edition. CRC Press, 2002.

[44] J. D. Vincent, et al. Fundamentals of Infrared and Visible Detector Operation and Testing, $2^{\text {nd }}$ Ed., John Wiley and Sons, 2015.

[45] J. Grayer and S.-S. Yoo, "Investigation of nanoplasmonic antenna for increased performance of high temperature operation PbSe photoconductors," Proceedings of SPIE, Vol. 10722, 2018. 


\section{Chapter 4: Thin Film Interference and the Back Reflector}

\subsection{Derivation of the Refractive Index and Absorption Coefficient}

In Chapter 3, the interaction of light with matter was explained from the perspective of the plasma frequency in metals. The discussion is now expanded to the provenance of the refractive index of materials. Consider a beam of light incident on a semiconductor material. When the light wave interacts with the particles in the film there is an induced dipole moment due to the oscillation of the incident electric field. Let the variable $\mathbf{P}$ represent this resulting polarization from the induced dipole, such that the effective charge $\rho$ and the current density are given by equations 4.1 and 4.2 , respectively.

$$
\begin{gathered}
\rho=-\nabla \cdot \boldsymbol{P} \\
\boldsymbol{j}=\frac{\partial \boldsymbol{P}}{\partial t}
\end{gathered}
$$

Accounting for polarization effects, Maxwell's equations are now given by equations 4.34.6,

$$
\begin{gathered}
\nabla \cdot \boldsymbol{E}=-\frac{(\nabla \cdot \boldsymbol{P})}{\varepsilon_{0}} \\
\nabla \times \boldsymbol{E}=-\frac{\partial \boldsymbol{B}}{\partial t} \\
\nabla \cdot \boldsymbol{B}=0 \\
c^{2} \nabla \times \boldsymbol{B}=\frac{\partial}{\partial t}\left(\frac{\boldsymbol{P}}{\varepsilon_{0}}+\boldsymbol{E}\right)
\end{gathered}
$$

where the variable for the speed of light, which can be found from the electric permittivity and magnetic susceptibility of free space by $c=\left(\varepsilon_{0} \mu_{0}\right)^{-1 / 2}$, is included to simplify the derivation [9]. By taking the curl of both sides in equations 4.4 and 4.6, the 
electric and magnetic fields can be decoupled leading to the wave equations for the electric and magnetic fields. The traveling wave solutions for the electric and magnetic fields take on the form of equations 4.7 and 4.8 ,

$$
\begin{aligned}
\boldsymbol{E} & =\boldsymbol{E}_{\mathbf{0}} \exp [i(\omega t-k \cdot r)] \\
\boldsymbol{H} & =\boldsymbol{H}_{\mathbf{0}} \exp [i(\omega t-k \cdot r)]
\end{aligned}
$$

where the variable $\mathrm{k}$ is the wave vector, the amplitude of which is the wave number given by $|k|=2 \pi / \lambda$, and the variable $\omega$ is the angular frequency given as $2 \pi f[1-5]$. The argument of the exponential of these solutions is what is known as the phase of the individual wave, and leads to the phase velocity $v_{p}$ and group velocity $v_{g}$, shown in equations 4.9 and 4.10 , of the waves propagating in the material.

$$
\begin{aligned}
& v_{p}=\frac{\omega}{k} \\
& v_{g}=\frac{\partial \omega}{\partial k}
\end{aligned}
$$

The investigated PbSe films are absorptive, so the wave vector will be complex taking the form $\tilde{k}=k_{1}+i k_{2}[5]$. The index of refraction is a measure of the reduction of the speed of light as it travels through a medium [5-7]. Examining equation 4.9, the phase velocity is related to the speed of light by equation 4.11 .

$$
\frac{\omega}{k}=\frac{c}{n}
$$

Therefore, the complex index of refraction for absorptive mediums is given by equation 4.12 .

$$
m=\tilde{k}\left(\frac{c}{\omega}\right)=\frac{k_{1}}{|\tilde{k}|}+\frac{i k_{2}}{|\tilde{k}|}=n+i \kappa
$$


Equation 4.12 shows that the real part of the refractive index is equivalent to the real part of the wave vector normalized to its magnitude, and the imaginary part of the refractive index is the imaginary part of the wave vector normalized to its magnitude. From this relationship, it is clear that the dispersion relations relating the complex index of refraction to the incident wavelength are equivalent to the dispersion relations that relate the complex wave vector to angular frequency. There is another relationship to be considered, which relates the time variation and the spatial variation of the incident wave according to equation 4.13 .

$$
k^{2}=\varepsilon\left(\frac{\omega}{c}\right)^{2}
$$

From equations 4.11 and 4.13 , the complex index of refraction $m$ is the square root of the complex permittivity $\varepsilon$. To further define the components of each, the real part of the complex permittivity can be found by equation 4.14 ,

$$
\varepsilon_{1}=n^{2}-\kappa^{2}
$$

and the imaginary part can be found by equation 4.15 .

$$
\varepsilon_{2}=2 n \kappa
$$

These relationships have been used extensively throughout this study, examples of which will be discussed in Chapter 5 .

With the components of the complex index of refraction defined, the absorption coefficient $\alpha$, the square root of which is proportional to detector sensitivity from equation 1.2 , can be defined. As stated previously, the imaginary part of the refractive index stems from the attenuation of light as it passes through an absorptive medium. So, 
for the imaginary part of the index of refraction, the phase velocity can be rearranged to yield $\alpha$ by equation 4.16 .

$$
\alpha=\frac{2 \omega \kappa}{c}=\frac{4 \pi \kappa}{\lambda}
$$

\subsection{Absorption in PbSe Thin Films}

Table 6. Calculated absorption coefficient using the dispersion relations from figure 28 for PbSe films.

\begin{tabular}{|c|c|c|c|c|c|c|c|}
\hline $\begin{array}{c}\text { Wavelength } \\
(\boldsymbol{\mu m})\end{array}$ & $\mathbf{n}$ & $\mathbf{k}$ & $\begin{array}{c}\boldsymbol{\alpha} \\
\left(\mathbf{c m}^{-1}\right)\end{array}$ & $\begin{array}{c}\text { Wavelength } \\
(\boldsymbol{\mu m})\end{array}$ & $\mathbf{n}$ & $\mathbf{k}$ & $\begin{array}{c}\boldsymbol{\alpha} \\
\left(\mathbf{c m}^{-\mathbf{1}}\right)\end{array}$ \\
\hline 2.166 & 4.7 & 0.25 & 14504.12 & 3.730 & 4.95 & 0.22 & 7412.09 \\
\hline 2.237 & 4.7 & 0.254 & 14265.43 & 3.836 & 4.95 & 0.199 & 6519.07 \\
\hline 2.441 & 4.7 & 0.238 & 12251.93 & 3.950 & 4.96 & 0.194 & 6172.30 \\
\hline 2.686 & 4.7 & 0.45 & 21054.37 & 4.069 & 4.97 & 0.189 & 5837.17 \\
\hline 2.797 & 4.9 & 0.4 & 17968.71 & 4.197 & 4.98 & 0.173 & 5180.31 \\
\hline 2.919 & 4.88 & 0.25 & 10763.73 & 4.332 & 4.97 & 0.127 & 3684.05 \\
\hline 2.984 & 4.883 & 0.22 & 9265.79 & 4.630 & 4.95 & 0.0572 & 1552.53 \\
\hline 3.052 & 4.885 & 0.22 & 9058.64 & 4.796 & 4.94 & 0.0353 & 925.01 \\
\hline 3.197 & 4.895 & 0.22 & 8647.44 & 4.973 & 4.94 & 0.00917 & 231.71 \\
\hline 3.275 & 4.9 & 0.22 & 8441.55 & 5.165 & 4.94 & 0.00285 & 69.34 \\
\hline 3.357 & 4.91 & 0.22 & 8234.60 & 5.371 & 4.92 & 0.00154 & 36.03 \\
\hline 3.443 & 4.85 & 0.219 & 7993.46 & 5.595 & 4.9 & 0.00138 & 31.00 \\
\hline 3.534 & 4.93 & 0.225 & 8001.05 & 5.838 & 4.83 & 0.00128 & 27.55 \\
\hline
\end{tabular}

Using the dispersion relations found in figure 28 , the wavelength dependent absorption coefficient at room temperature for the investigated $\mathrm{PbSe}$ films was calculated. Table 6 and figure 37 show the calculated results for the room temperature absorption coefficient between approximately $2 \mu \mathrm{m}$ and $6 \mu \mathrm{m}$. The calculated MWIR absorption coefficient for the investigated films agrees well with the previously reported value of $10000 \mathrm{~cm}^{-1}$ by Baleva [8]. 


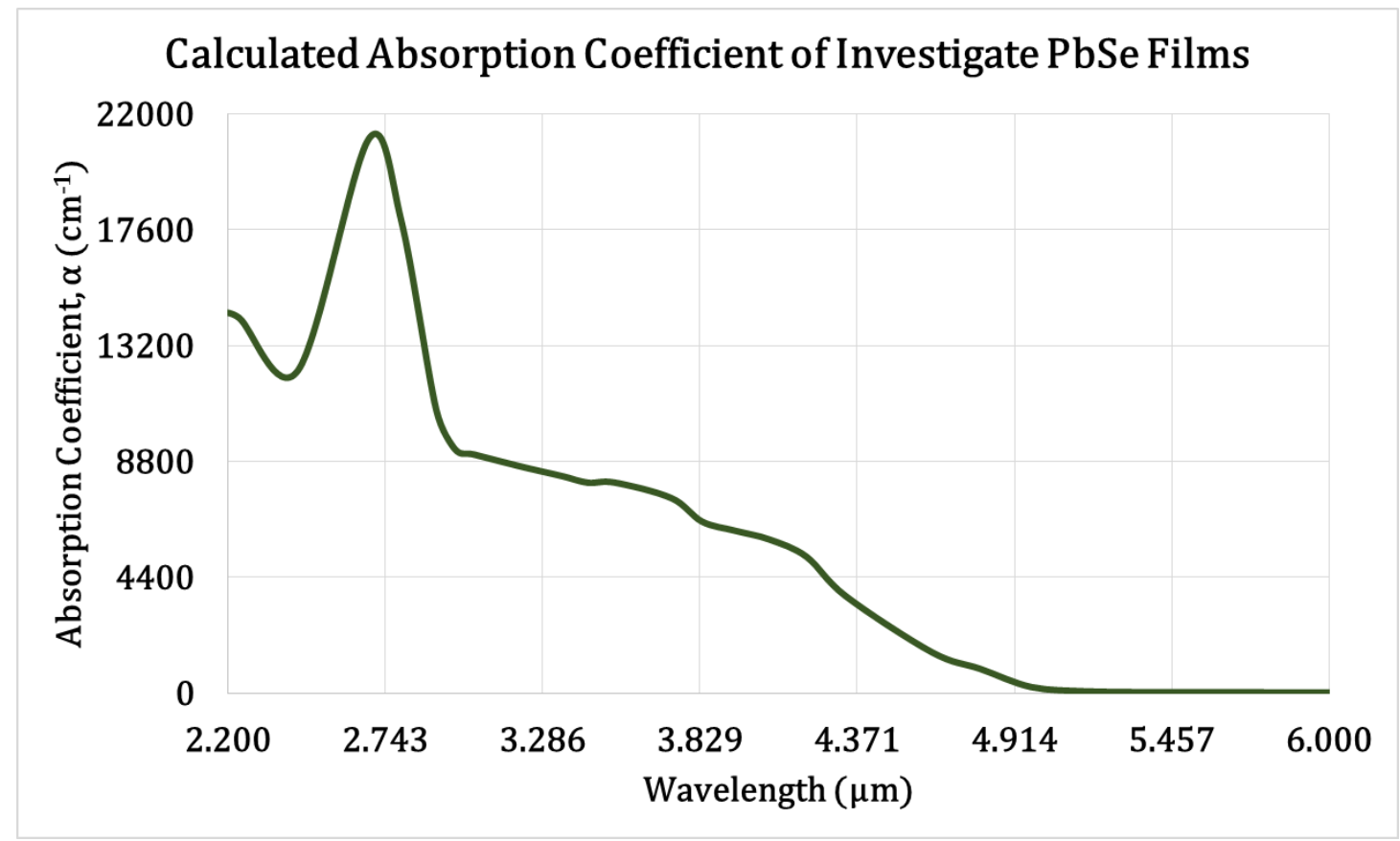

Figure 37. Calculated wavelength-dependent absorption coefficient of PbSe films.

As seen in figure 37 , the absorption coefficient between $3 \mu \mathrm{m}$ and $5 \mu \mathrm{m}$ is weighted toward lower wavelengths. The Beer-Lambert law, seen in equation 4.17, describes the resulting field intensity $I$ of a wave after it propagates through a finite distance within a medium,

$$
I=I_{0} e^{-\alpha t}
$$

where $I_{0}$ is the incident field intensity in units of $\mathrm{W} / \mathrm{cm}^{2}$, and $\mathrm{t}$ is the thickness through which the wave propagates in $\mathrm{cm}$ [5]. Dividing the resulting field intensity by the incident field intensity yields the transmission, which can be used to find the absorption within the material by equation 4.18 .

$$
A=1-\frac{I}{I_{0}}=1-e^{-\alpha t}
$$




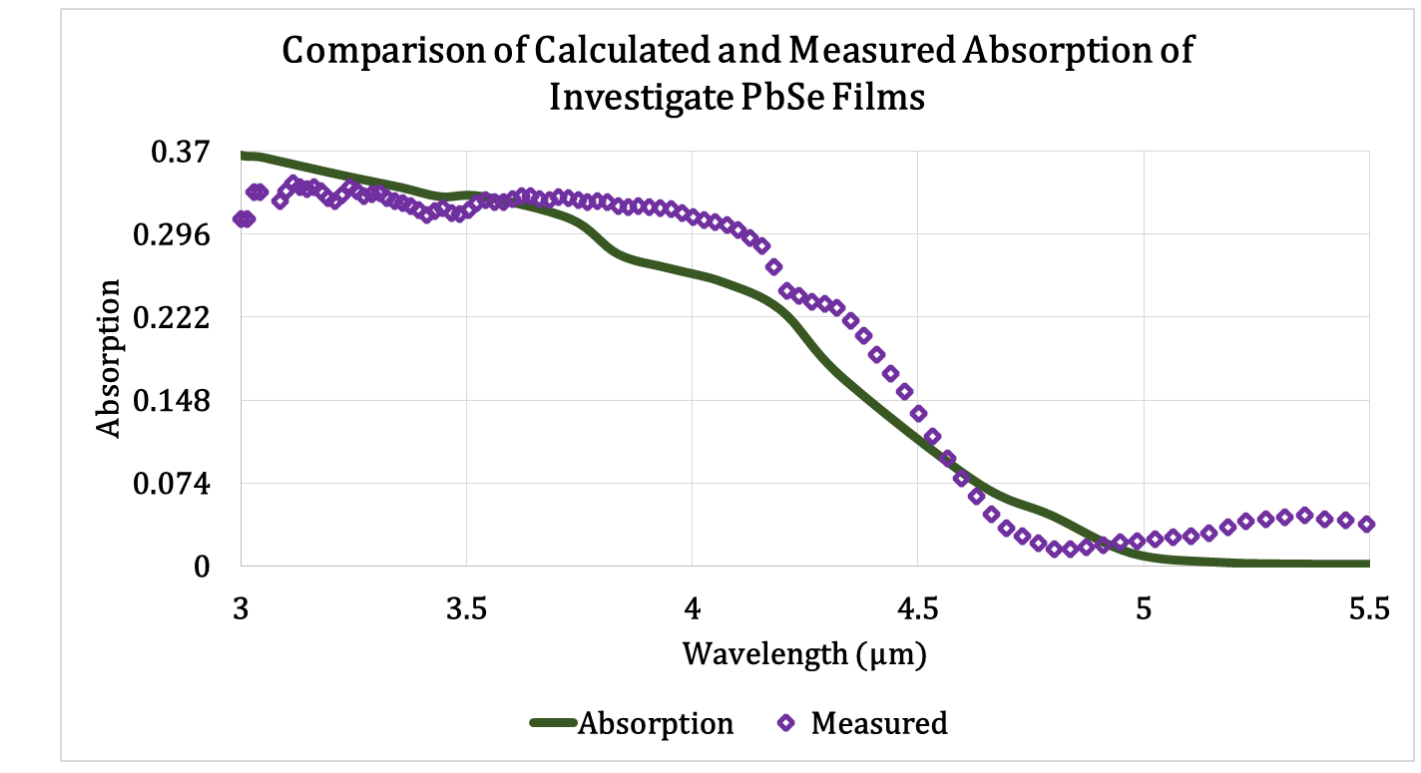

Figure 38. Comparison of absorption calculated from modified PbSe dispersion relations and measured absorption in multi-layer PbSe films.

Stated in Chapter 2, the investigated PbSe films of this study are grown to a thickness of approximately $1 \mu \mathrm{m}$, half of which is converted to $\mathrm{PbI}_{2}$ after sensitization. Therefore, using equation 4.18 and the wavelength-dependent absorption coefficient found in table 6, I calculated the absorption within PbSe films. Shown in figure 38, the absorption of PbSe was calculated to be $\sim 25-37 \%$ above the absorption edge. Included in figure 38 is the measured absorption spectrum from figure 20 for comparison. The calculated absorption agrees well with that of the measured up to $\sim 3.6 \mu \mathrm{m}$, yet between $3.7 \mu \mathrm{m}$ and $4.5 \mu \mathrm{m}$ the measured spectrum has a higher absorption. The calculated spectrum of figure 38 only takes into consideration the dispersion relations of $\mathrm{PbSe}$, yet the films I investigated were engineered to contain multiple layers of alternating values of the index of refraction. As mentioned in Chapter 2, the $\mathrm{PbI}_{2}$ and $\mathrm{SiO}_{2}$ layers, as well as the Si substrate, are wide bandgap semiconductors, so are considered to be transparent to MWIR radiation. However, from figure 38 it is clear that these layers have an effect on 
the absorption of the films that were investigated. Previously described in Chapter 1, this multi-layer structure leads to constructive interference which increases the absorption for wavelengths close to $4 \mu \mathrm{m}$ in the investigated films. The origin of the interference in multi-layer thin films will now be discussed.

\subsection{Thin Film Interference}

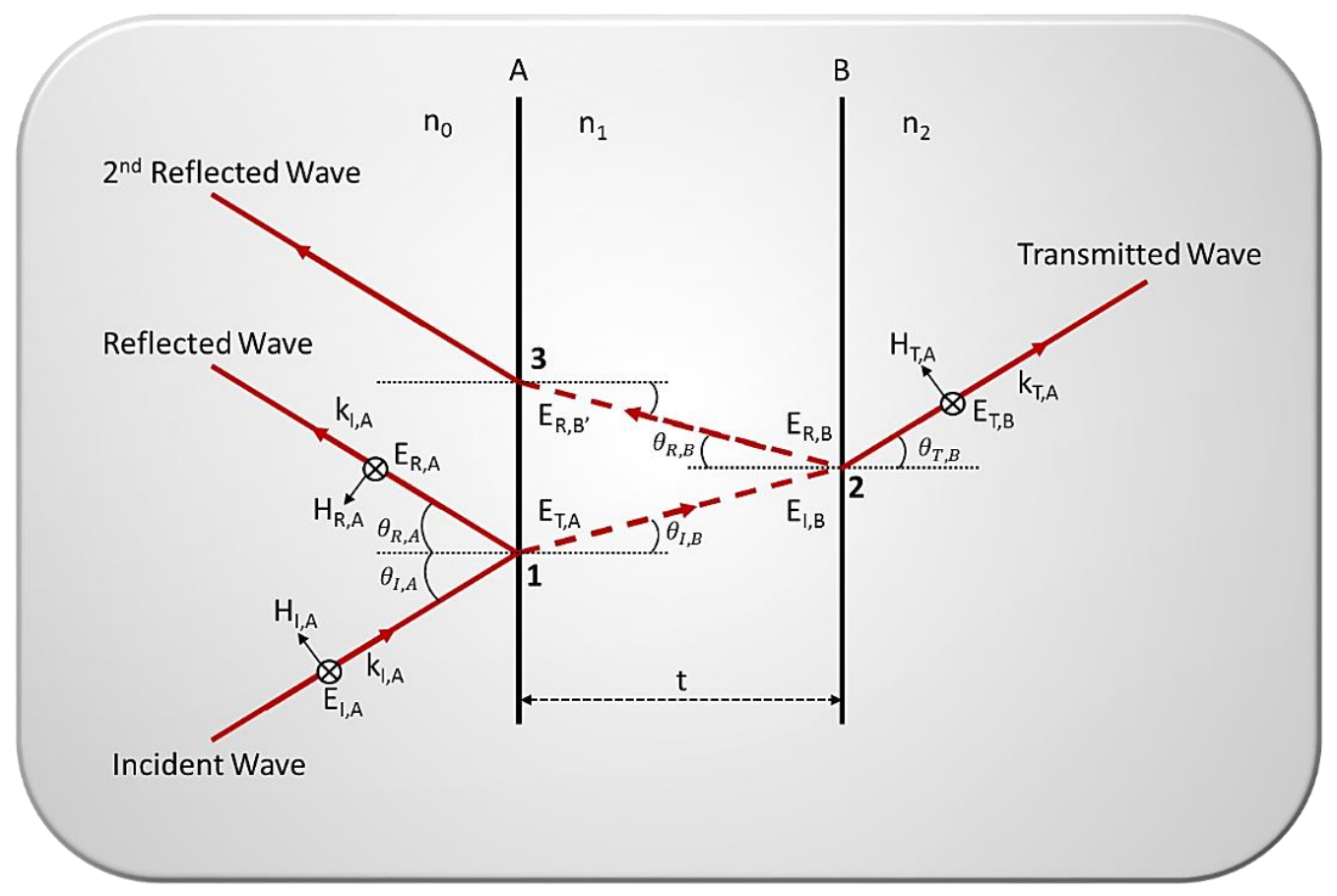

Figure 39. Interaction of a light wave as it transmits through the layers of an optical thin film.

Consider the situation presented in figure 39 in which an incident light wave travels within a three-layer thin film. The index of refraction of the three layers are labeled as $\mathrm{n}_{0}$ for the incident medium which is considered to be air, $\mathrm{n}_{1}$ for the intermediate medium, and $\mathrm{n}_{2}$ for the final medium, which is considered to be a thick substrate. The electrodynamic behavior within the thin film leads to the phenomenon of reflection, transmission, and absorption $[2,5]$. Let $A$ represent the interface between medium 1 and medium 2, while $B$ represents the interface between medium 2 and the 
substrate. From the continuity condition of Maxwell's equations the tangential part of the electric and magnetic fields must be continuous across the boundary [5]. However, the normal part of the electric field is not continuous across the interface, due to the differences in the polarizabilities between materials on either side of the boundary. Instead the electric displacement is conserved [9]. Using the continuity condition, and utilizing the propagation vector $\boldsymbol{H}=\sqrt{\frac{\varepsilon_{0}}{\mu_{0}}}(n \boldsymbol{k} \times \boldsymbol{E})$, the electric $\mathrm{E}_{\mathrm{A}}$ and magnetic $\mathrm{H}_{\mathrm{A}}$ field components at $A$ in figure 39 are given by equations 4.19 and 4.20 ,

$$
\begin{gathered}
E_{A}=E_{I, A}+E_{R, A}=E_{T, A}+E_{R^{\prime}, B} \\
H_{A}=\sqrt{\frac{\varepsilon_{0}}{\mu_{0}}}\left(E_{I, A}-E_{R, A}\right) n_{0} \cos \theta_{I, A}=\sqrt{\frac{\varepsilon_{0}}{\mu_{0}}}\left(E_{T, A}-E_{R^{\prime}, B}\right) n_{1} \cos \theta_{I, B}
\end{gathered}
$$

where $\mathrm{E}_{\mathrm{I}, \mathrm{A}}, \mathrm{E}_{\mathrm{R}, \mathrm{A}}$, and $\mathrm{E}_{\mathrm{T}, \mathrm{A}}$ represent the incident, reflected, and transmitted tangential electric field component at interface $A$. The quantity $\mathrm{E}_{\mathrm{R}}{ }^{\prime}, \mathrm{B}$ represents the tangential electric field component reflected from $B$, while the $\mathrm{n}_{0}$ and $\mathrm{n}_{1}$ are the refractive indices of the incident medium and the single layer film [5]. The prime denotation for the reflected part of the field is to indicate that the component is to be considered at interface $A$, which is to say it is the reflected component after passing through the film from $A$ to $B$. The PbSe films and devices we investigated were designed to be illuminated at or near normal incidence, so the term $\cos \theta_{I, A}$ can be considered to be 1 . The refracted angle $\theta_{B}$ for the ideal case would also be normal to the film surface, however in real films significant scattering can occur which would increase the contribution from other angles of illumination.

Similarly, the components of the tangential part of the electric $\mathrm{E}_{\mathrm{B}}$ and magnetic $\mathrm{H}_{\mathrm{B}}$ fields are given by equations 4.21 and 4.22 , 


$$
\begin{gathered}
E_{B}=E_{I, B}+E_{R, B}=E_{T, B} \\
H_{B}=\sqrt{\frac{\varepsilon_{0}}{\mu_{0}}}\left(E_{I, B}-E_{R, B}\right) n_{1} \cos \theta_{I, B}=\sqrt{\frac{\varepsilon_{0}}{\mu_{0}}} E_{T, B} n_{2} \cos \theta_{T, B}
\end{gathered}
$$

where $\mathrm{E}_{\mathrm{R}, \mathrm{B}}$ is the component reflected from and in the vicinity of $B, \mathrm{n}_{2}$ is the refractive index of the substrate or subsequent layer, and $\theta_{T, B}$ is the angle at which the beam refracts into the substrate.

Recall that the vacuum wavelength of light is reduced by the index of refraction of the medium in which it propagates. To determine if the first and second reflected waves interfere, consider the optical path length the original wave traverses as it propagates through the film. Referring to figure 39 , let the point in which the incident ray enters the medium of index $\mathrm{n}_{1}$ be denoted as $\mathbf{1}$, the point in which the refracted wave enters the medium with refractive index of $\mathrm{n}_{2}$ be known as $\mathbf{2}$, and finally the point at which the second reflected wave exits the medium and enters the incident medium be $\mathbf{3}$. The optical path length $\triangle P L$ is given by equation 4.23 ,

$$
\Delta P L=\frac{|\overrightarrow{12}|+|\overrightarrow{23}|}{\lambda_{n}}=\frac{n(|\overrightarrow{12}|+|\overrightarrow{23}|)}{\lambda}
$$

where $|\overrightarrow{12}|$ and $|\overrightarrow{23}|$ represent the length of the vectors between the points $\mathbf{1}$ and $\mathbf{2}$ and $\mathbf{2}$ and $\mathbf{3}$, respectively [10]. In the special case of normal incidence, the sum of the length of the vectors between points $\mathbf{1}, \mathbf{2}$, and $\mathbf{3}$ is just double the thickness t. Thus, $\triangle P L$ then reduces to equation 4.24 .

$$
\Delta P L=\frac{2 n t}{\lambda}
$$

The numerator in equation 4.24 is known as the optical path length, which is used to determine whether interference will be constructive or destructive. The optical path 
length is only part of the picture, however. Above, it was stated that the first wave will undergo a $180^{\circ}$ phase shift upon reflection. This can be regarded as an equivalent path difference of $\Delta R=\lambda_{0} / 2$, which must be taken into account. Including this phase shift, the conditions for constructive interference are given by equation 4.25 , and those for destructive interference given by equation 4.26 .

$$
\begin{aligned}
& \Delta P L+\Delta R=p \lambda ;(p=1,2,3, \ldots) \\
& \Delta P L+\Delta R=p \lambda ;\left(p=\frac{1}{2}, \frac{3}{2}, \frac{5}{2} \ldots\right)
\end{aligned}
$$

The importance of equation 4.25 and 4.26 with respect to the PbSe films of this study should be evident. When a light beam travels through the PbSe film, interference can occur due to the layers containing media of alternating high and low refractive index. In fact, this phenomenon can be exploited to increase the absorption and field intensity within the PbSe layer.

Returning to the light wave as it propagates through the film, a phase shift, shown in equation 4.27 , will occur as the wave propagates through the film,

$$
\frac{k_{0}\left(2 n_{1} t \cos \theta_{I, B}\right)}{2}=k_{0} g
$$

where the variable $g$ has been used to simplify the parenthetical argument. The incident and reflected waves at B can then be defined in terms of the transmitted wave and reflected wave from B at A by equations 4.28 and 4.29.

$$
\begin{gathered}
E_{I, B}=E_{T, B} e_{0}^{-i k g} \\
E_{R, B}=E_{R^{\prime}, B} e^{i k_{0} g}
\end{gathered}
$$

The fields at interface A and interface B can now be described as in equations $4.30-4.33$. 


$$
\begin{gathered}
E_{B}=E_{T, A} e^{-i k_{0} g}+E_{R^{\prime}, B} e^{i k_{0} g} \\
H_{B}=\left(E_{T, A} e^{-i k_{0} g}-E_{R^{\prime}, B} e^{i k_{0} g}\right) \sqrt{\frac{\varepsilon_{0}}{\mu_{0}}} n_{1} \cos \theta_{I, B} \\
E_{A}=E_{B} \cos k_{0} g+\frac{H_{B}\left(i \sin k_{0} g\right)}{\sqrt{\frac{\varepsilon_{0}}{\mu_{0}}} n_{1} \cos \theta_{I, B}} \\
H_{A}=E_{B} \sqrt{\frac{\varepsilon_{0}}{\mu_{0}}} i \sin k_{0} g+H_{B} \cos k_{0} g
\end{gathered}
$$

It is often more efficient to treat these equations in matrix form, especially when extending to multiple layers $[5,9]$. The above relationships in matrix form are given by equation 4.34 .

$$
\left[\begin{array}{c}
E_{A} \\
H_{A}
\end{array}\right]=\left[\begin{array}{cc}
\cos _{0} g & \frac{i \operatorname{sing} k_{0} g}{\operatorname{sqrt}\left(\frac{\varepsilon_{0}}{\mu_{0}}\right) n_{1} \cos \theta_{I, B}} \\
\sqrt{\frac{\varepsilon_{0}}{\mu_{0}}} \text { isink }_{0} g & \cos _{0} g
\end{array}\right]\left[\begin{array}{l}
E_{B} \\
H_{B}
\end{array}\right]
$$

The transformation matrix that relates fields at the second interface to fields at the first is known as the characteristic matrix, which will be denoted as $\mathrm{M}_{j}$ where the subscript $\mathrm{j}$ represents the layer under evaluation. The characteristic matrix of a single layer has been given, however the method can be extended to a q-layer film with equations 4.35 and 4.36 .

$$
\begin{gathered}
M_{\text {film }}=\prod_{j=1}^{q} M_{j}=M_{1} M_{2} \cdots M_{q}=\left[\begin{array}{ll}
m_{11} & m_{12} \\
m_{21} & m_{22}
\end{array}\right] \\
{\left[\begin{array}{l}
E_{A} \\
H_{A}
\end{array}\right]=M_{\text {film }}\left[\begin{array}{l}
E_{q+1} \\
H_{q+1}
\end{array}\right]}
\end{gathered}
$$

Returning to the single layer film, it is now possible to determine the reflection and transmission amplitude coefficients in terms of the components of the light wave [5, 9]. The matrix form of equations 4.21 and 4.22 is given by equation 4.37 . 


$$
\left[\begin{array}{c}
E_{I, A}+E_{R, A} \\
\left(E_{I, A}-E_{R, A}\right) \sqrt{\frac{\varepsilon_{0}}{\mu_{0}}} n_{0} \cos \theta_{I, A}
\end{array}\right]=M_{1}\left[\begin{array}{c}
E_{T, B} \\
E_{T, B} \sqrt{\frac{\varepsilon_{0}}{\mu_{0}}} n_{2} \cos \theta_{T, B}
\end{array}\right]
$$

Let $\tilde{r}$ and $\tilde{t}$ be the reflection and transmission amplitude coefficients, respectively. Relating these coefficients to the incident, reflected, and transmitted waves while expanding equation 4.37 leads to equations 4.38 and 4.39 .

$$
\begin{gathered}
\tilde{r}=\frac{\left(\sqrt{\frac{\varepsilon_{0}}{\mu_{0}}} n_{0} \cos \theta_{I, A} m_{11}+\frac{\varepsilon_{0}}{\mu_{0}} n_{0} n_{2} \cos \theta_{I, A} \cos \theta_{T, B}-m_{21}-\sqrt{\frac{\varepsilon_{0}}{\mu_{0}}} n_{2} \cos \theta_{T, B} m_{22}\right)}{\sqrt{\frac{\varepsilon_{0}}{\mu_{0}}} n_{0} \cos A m_{11}+\frac{\varepsilon_{0}}{\mu_{0}} n_{0} n_{2} \cos \theta_{I, A} \cos \theta_{T, B} m_{12}+m_{21}+\sqrt{\frac{\varepsilon_{0}}{\mu_{0}}} n_{2} \cos \theta_{T, B} m_{22}} \\
\tilde{t}=\frac{2 \sqrt{\frac{\varepsilon_{0}}{\mu_{0}}} n_{0} \cos \theta_{I, A}}{\sqrt{\frac{\varepsilon_{0}}{\mu_{0}}} n_{0} \cos A m_{11}+\frac{\varepsilon_{0}}{\mu_{0}} n_{0} n_{2} \cos \theta_{I, A} \cos \theta_{T, B} m_{12}+m_{21}+\sqrt{\frac{\varepsilon_{0}}{\mu_{0}}} n_{2} \cos \theta_{T, B} m_{22}}
\end{gathered}
$$

For larger angles of incidence, the reflectance and transmittance amplitude coefficients can be very complex, however typical infrared detection and imaging systems are designed to collect light at small angles near normal incidence. This greatly reduces the complexity of equations 4.38 and 4.39. As an example, for a single layer film assuming normal incidence, equation 4.38 reduces to equation 4.40 ,

$$
\tilde{r}=\frac{n_{1}\left(n_{0}-n_{2}\right) \cos _{0} g+i\left(n_{0} n_{2}-n_{1}^{2}\right) \sin _{0} g}{n_{1}\left(n_{0}+n_{2}\right) \cos k_{0} g+i\left(n_{0} n_{2}+n_{1}^{2}\right) \sin _{0} g}
$$

where $\mathrm{n}_{2}$ in this case is the substrate refractive index. Finally, the square of the amplitude coefficients gives the overall reflectance $\mathrm{R}$ and transmittance $\mathrm{T}$ of the film. Therefore, the reflectance of the film is given by equation 4.41 ,

$$
R_{1}=r_{1} r_{1}^{*}=\frac{n_{1}^{2}\left(n_{0}-n_{2}\right)^{2} \cos ^{2} k_{0} g+\left(n_{0} n_{2}-n_{1}^{2}\right) \sin ^{2} k_{0} g}{n_{1}^{2}\left(n_{0}+n_{2}\right)^{2} \cos ^{2} k_{0} g+\left(n_{0} n_{2}+n_{1}^{2}\right)^{2} \sin ^{2} k_{0} g}
$$

where the $*$ denotation represents the complex conjugate operator. Depending on the optical thickness of the film, $\mathrm{R}$ can be further simplified. This simplification is used in 
thin film applications like interference filters, anti-reflection coatings, and dielectric mirrors [11-17].

\subsection{The Back Reflector Structure in PbSe}

Discussed in Chapter 1, interference in the investigated PbSe films is possible in the $\mathrm{PbSe}$ and $\mathrm{PbI}_{2}$ layers. The majority of the field intensity enhancement, which directly effects the absorption, from constructive interference will occur within the PbSe layer. It should be noted, however, that the films are sufficiently thin that interference fringes generated in the $\mathrm{PbI}_{2}$ will reach into PbSe offering a small enhancement [5]. From calculations shown in Chapter 1 , I found that $23 \%$ of the incident light will transmit through the film into the substrate without being absorbed or reflected. Though the situation presented in figure 2 assumes smooth layers and sharp interfaces, its argument is still valid for the rough films that were investigated. Indeed, figure 38 demonstrates the effects of interference in these multilayer films, yet up to $23 \%$ of the incident light is lost to transmission. The intensity of the resultant interference wave is directly related to the amplitudes of the constituent waves of which it is comprised [5, 9-10]. Therefore, the transmission loss through the substrate leads to reduced electric field intensity in the interference fringes created in the PbSe layer. The addition of an embedded metal reflector, in this case platinum, can help to circumvent this transmission loss by reflecting all of the light that transmits through the film without absorption or reflection back into the film. The back reflector is attractive as a PbSe enhancement mechanism due to its ease of fabrication, wide area coverage, wavelength selectivity, and large increase in absorption and field intensity [18-21]. 
Shown in figure 40, the difference in the PbSe structure with an embedded $\mathrm{Pt}$ reflector is minimal. The $\mathrm{SiO}_{2}$ layer serves as a dielectric spacer with which to change the thickness of the film to selectively tune the resonant enhancement. The effective structure is that of a Fabry-Perot cavity [5, 10].

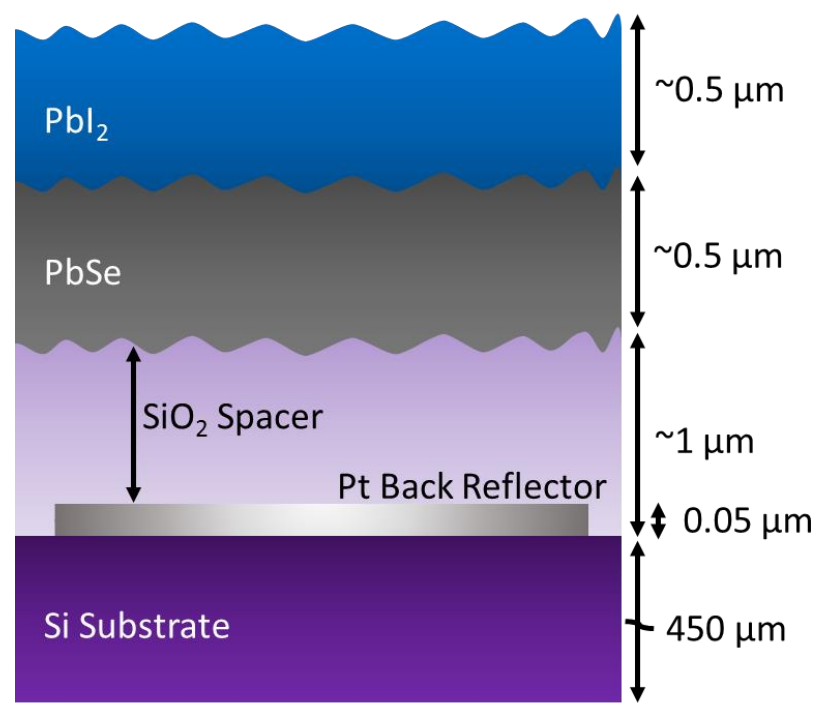

Figure 40. Depiction of PbSe film cross-section with embedded Pt back reflector.

Table 7. Layer thicknesses for modeled PbSe films with Pt back reflector.

\begin{tabular}{|c|c|c|}
\hline Layer & Refractive Index, $\mathbf{n}$ & Thickness (nm) \\
\hline $\mathrm{PbI}_{2}$ & 2.5 & 400 \\
\hline $\mathrm{PbSe}^{\mathrm{SiO}}{ }_{2}$ & 4.97 & 500 \\
\hline $\mathrm{Pt}$ & 1.39 & $800-1400$ \\
\hline $\mathrm{Si}$ substrate & 3.23 & 50 \\
\hline
\end{tabular}

As an example, consider a PbSe film with an embedded Pt back reflector. The metal of choice would typically be gold due to its high reflectivity and low losses in the MWIR regime. However, our experiments have shown that gold diffuses into the PbSe film during the oxidation and sensitization processes. Therefore, $\mathrm{Pt}$ was selected due to 
its high melting temperature, and high reflectivity in the MWIR regime [22]. As an aside, a popular configuration for commercial detectors is to back side illuminate them, such that the incident light transmits through the substrate instead of the top of the film. In this configuration, a dielectric spacer and back reflector could be deposited on the $\mathrm{PbI}_{2}$ layer after the sensitization process, and gold could be used. However, for this study the films and devices were designed for front side illumination.

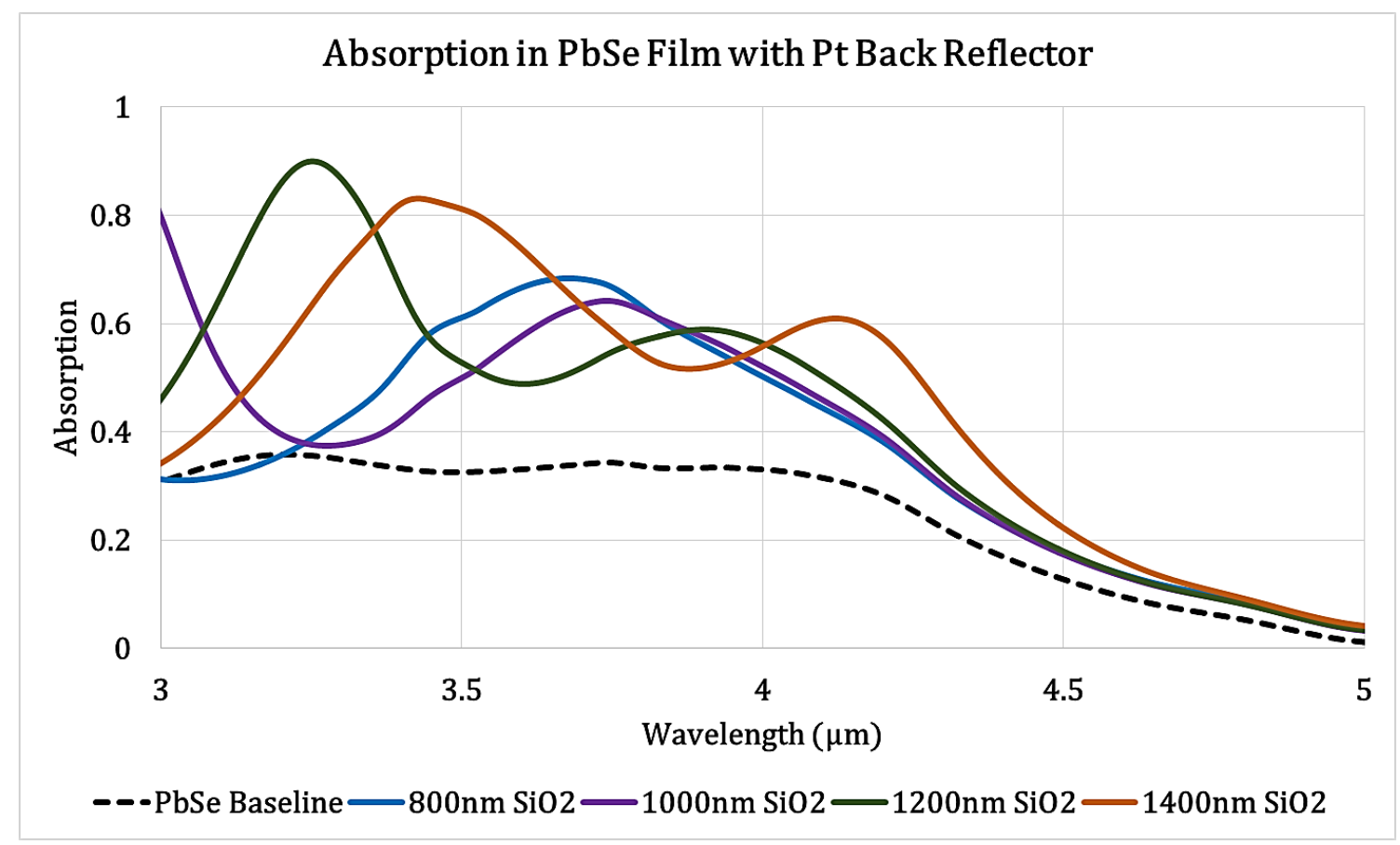

Figure 41. Calculated absorption in modeled PbSe films with Pt back reflector for various $\mathrm{SiO}_{2}$ thicknesses.

To first order, the film can be modeled numerically using the transmittance and reflectance coefficients derived previously. I modeled PbSe films with OpenFilters, an open-source thin film program from Montreal Polytechnique [23]. The $\mathrm{SiO}_{2}$ layer was varied between $800 \mathrm{~nm}$ to $1400 \mathrm{~nm}$ to observe how the spectrum shifts with dielectric spacer thickness. The layer thicknesses for the modeled films are shown in table 7. 
The calculated absorption in the modeled films is shown in figure 41 . The baseline PbSe film absorption (black dashed trace), shown here for reference, agrees well with the measured results of figure 20. For $\mathrm{SiO}_{2}$ thicknesses of $800 \mathrm{~nm}$ (blue trace) and $1000 \mathrm{~nm}$ (violet trace), single resonance peaks are observed at $3.64 \mu \mathrm{m}$ and $3.76 \mu \mathrm{m}$, respectively. As the $\mathrm{SiO}_{2}$ thickness is increased, the single-peaked spectra of $800 \mathrm{~nm}$ and $1000 \mathrm{~nm}$ become two-peaked spectra for $1200 \mathrm{~nm}$ (green trace) and $1400 \mathrm{~nm}$ (orange trace). Further, the absorption spectrum red-shifts with $\mathrm{SiO}_{2}$ thickness, moving one of the resonance peaks to coincide with the absorption edge of $\mathrm{PbSe}$. The presence of multiple peaks and the red-shift are indicative of interference within the films. The $\mathrm{SiO}_{2}$ spacer changes the length of the cavity setup within the film. When the thickness of the spacer is such that the cavity length corresponds to a longitudinal mode, optical interference will occur and increase the electric field intensity within the films [5]. These results demonstrate that by changing the $\mathrm{SiO}_{2}$ thickness, the absorption can be selectively tuned to the desired spectral region.

I also calculated the amplitude of the absorption enhancement, shown in figure 42, by dividing the absorption spectrum for each $\mathrm{SiO}_{2}$ thickness by that of the baseline $\mathrm{PbSe}$ film. Here, only the results between $3 \mu \mathrm{m}$ and $4.5 \mu \mathrm{m}$ are considered relevant, because the absorption is relatively low and falls off at longer wavelengths. The baseline trace, which represents the absorption in PbSe films without a back reflector, is shown as a black dashed line for reference. The results of these preliminary calculations were used to determine the optimum $\mathrm{SiO}_{2}$ thickness for back reflector enhanced PbSe devices. In this case, a $\mathrm{SiO}_{2}$ thickness of $1400 \mathrm{~nm}$ was found to be desirable due to the large peak at $3.45 \mu \mathrm{m}$ and the peak close to the PbSe absorption edge at $4.2 \mu \mathrm{m}$. Indeed, the $1400 \mathrm{~nm}$ 
$\mathrm{SiO}_{2}$ thickness yields an absorption as high as 2.55-times greater than that of baseline films, as well as a factor of two enhancement at the PbSe absorption edge. Described in Chapter 5, a second, higher fidelity optimization study was conducted utilizing finite element analysis to further refine these initial calculations before fabrication.

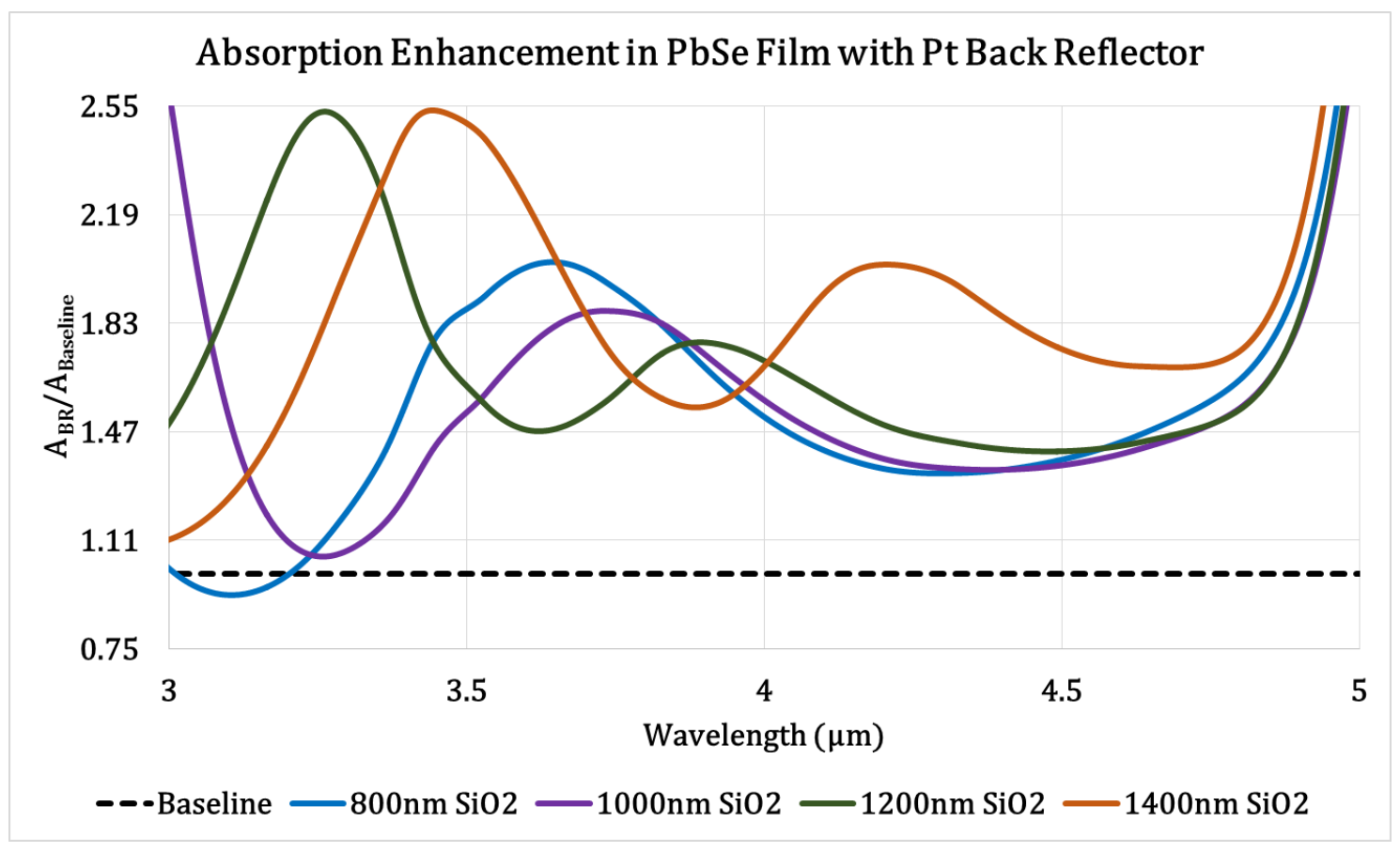

Figure 42. Calculated absorption enhancement in PbSe films with back reflector as a function of $\mathrm{SiO}_{2}$ thickness. 


\section{Chapter 4 References}

[1] J. D. Jackson, Classical electrodynamics, New York: Wiley, 1999.

[2] J. D. Griffiths, Introduction to electrodynamics, Upper Saddle River, N.J: Prentice Hall, 1999.

[3] C. A. Balanis. Advanced Engineering Electromagnetics, 1st Ed., Hoboken, NJ: John Wiley and Sons, Inc. 1989.

[4] D. Sarid and W. Challner, Modern Introduction to Surface Plasmons: Theory, Mathematical Modeling, and Application, New York, NY: Cambridge University Press, 2010.

[5] E. Hecht, Optics, 4th Edition. Addison-Wesley, New York, 2002.

[6] K. Kasunic, Optical Systems Engineering. McGraw-Hill Education, 2011.

[7] C. J. Willers, Electro-Optical Systems Analysis and Design, Bellingham, WA: SPIE Press, 2013.

[8] Baleva, et al. "Infrared Absorption of Laser Deposited PbSe Films," J. Physics C, Sol-State Phys., 1987.

[9] I. Chambouleyron and J. M. Marinez, Handbook of Thin Films Materials; ch 12, edited by Nalwa, H.S., Academic Press, 2001.

[10] OP-Tec: National Center for Optics and Photonics Education, Fundamental of Light and Lasers, 2nd ed. Texas: Op-TEC, 2013.

[11] M. S. Rauscher, et al. "Dual-channel mid-infrared sensor based on tunable FabryPerot filters for fluid monitoring applications," Sensors and Actuators B 259, 2018 
[12] E. Gayo, and J. Frutos, "Interference filters as an enhancement tool for infrared thermography in humidity studies of building elements," Infrared Physics and Technology 38, 1997.

[13] L. Fei, et al. "Design and fabrication of composite structure in ZnSe providing broadband mid-infrared anti-reflection,” Optical Materials 84, 2018.

[14] $\mathrm{H}$. Wu, et al. "High quantum efficiency N-structure type-II superlattice midwavelength infrared detector with resonant cavity enhanced design," Superlattices and Microstructures 105, 2017.

[15] F. Felder, et al. "Lead Salt Resonant Cavity Enhanced Detector with MEMS Mirror.” Physics Procedia 3, 2010.

[16] W. Heiss, et al. "Epitaxial Bragg mirrors for the mid-infrared and their applications.” Progress in Quantum Electronics 25, 2001.

[17] T. Wood, et al. "Study of the influence of temperature on the optical response of interferometric detectors systems," Sensors and Actuators A 203, 2013.

[18] S. Jain, et al. "Broadband absorption enhancement in ultra-thin crystalline Si solar cells by incorporating metallic and dielectric nanostructures in a back reflector," Pro. Photovolt: Res. Appl., Vol. 23, 2015.

[19] J. Piotrowski and A. Piotrowski, "Uncooled infrared photodetectors in Poland," Opto-Electron. Rev., 14, no. 1, 2006.

[20] J. J. Talghader, A. S. Gawarikar, and P. R. Shea, "Spectral selectivity in infrared thermal detection," Light: Science \& Applications, Vol. 1, 2012. 
[21] X. Tang, M. Ackerman, and P. Guyot-Sionnest, "Thermal Imaging with Plasmon Enhanced HgTe Collidal Quantum Dots Photovoltaic Devices," manuscript nn2018-03871g submitted to ACS Nano, 2018.

[22] A. Y-C. Yu and W. E. Spicer, "Optical Properties of Platinum," Physical Review, Vol. 171, No. 3, 1968.

[23] S. Larouche, and L. Martinu, "OpenFilters: open-source software for the design, optimization, and synthesis of optical filters," Appl. Optics, vol. 47, issue 13, 2008.

[24] Evan, Amato. University of California San Diego, Scripps Institute of Oceanography, Department of Atmospheric and Climate Sciences 251: Radiation The Atmosphere Ch3 Class Notes

[25] C. Matzler, "MATLAB Functions for Mie Scattering and Absorption," Institut fur Angewandte Physik, Research Report No 2002-08, 2002. 


\section{Chapter 5: Design Methodology and Performance Predictions}

We have shown that, depending on the type of resonant structure, there are many degrees of freedom with which to selectively enhance PbSe films. As we discussed in Chapter 3, with surface plasmon resonant structures the morphology, metal type, and surrounding material dictate the resonant condition. In regards to the back reflector structure discussed in Chapter 4, the metal type and dielectric spacer thickness determine the resonance. To narrow the design space, I employed a design methodology similar to that of Choi [1-3]. First, numerical analysis was carried out using MATLAB or OpenFilters to select design parameters. The chosen parameters were inserted into the finite element modeling (FEM) tool ANSYS HFSS to predict the absorption, as well as the field dynamics, in the PbSe films we investigated [4-10]. I started finite element modeling efforts with a single unit cell, the results of which were compared with those obtained with numerical methods for validation. Then, an infinite array of unit cells, meant to approximate the physical film, was modeled to determine the effect of the resonant structure on the film absorption. For optimization, I conducted parametric analyses of structure and film parameters, the results of which were used to choose the optimum design dimensions. Structures based on the chosen parameters were fabricated with help from my collaborators, and incorporated into our $\mathrm{PbSe}$ photoconductive detectors. I conducted detector measurements, and compared the results to my models for concurrence. Moreover, I compared the resonant structure enhanced $\mathrm{PbSe}$ photoconductor measurements to the reference detector measurements, shown in Chapter 2, to verify we had achieved the goal of raising the operating temperature of $\mathrm{PbSe}$ 
detectors. These last two steps will be described in Chapter 6. In this chapter, the design and optimization of the resonant structure enhanced films will be discussed in detail.

\subsection{FEM and the ANSYS HFSS Environment}

The necessary theoretical background for resonant structures based on surface plasmon resonance and interference effects were discussed in Chapter 3 and Chapter 4. The numerical models I constructed and employed for this investigation follow closely with the theoretical background, and thus will not be discussed here. Instead, attention is turned to the finite element analysis method and the ANSYS HFSS simulation environment.

Finite element modeling (FEM) is a powerful tool for solving Maxwell's equations for complex objects [11-12]. The solution to these relationships can become intractable, requiring large computing resources and time to produce meaningful results. The FEM method is attractive due to its capability of solving nonhomogeneous regions quickly and efficiently.
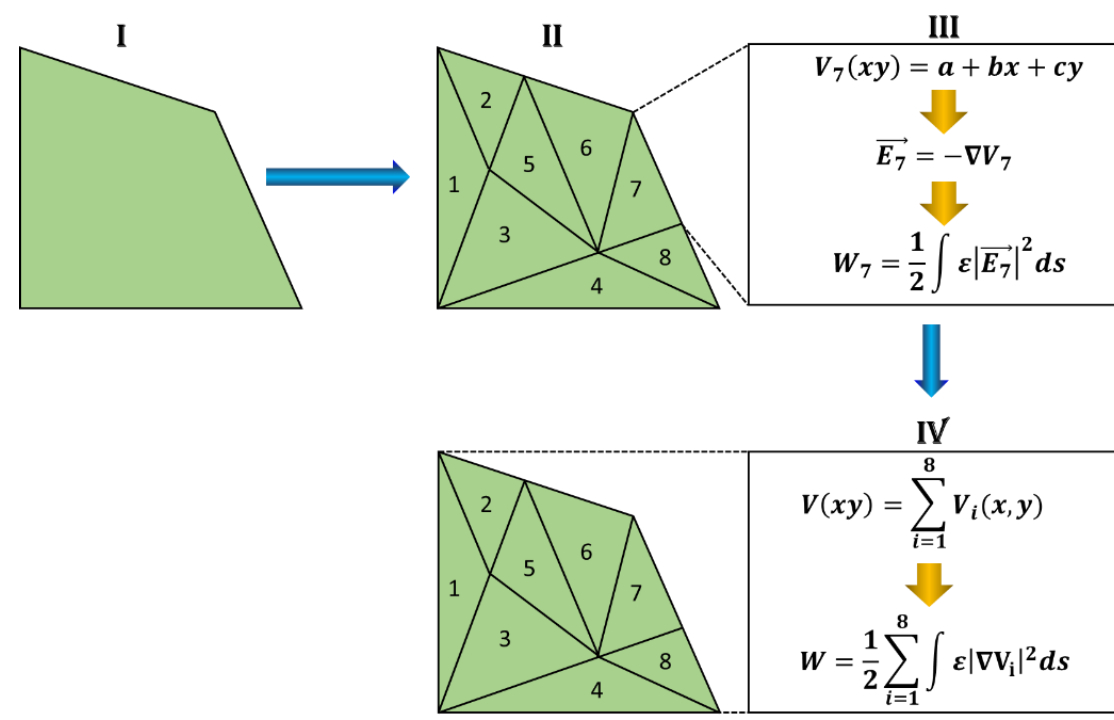

Figure 43. Simple depiction of the finite element method (FEM) for solving electromagnetic problems. 
Depicted in figure 43, there are four major steps in the FEM method [12]. In step I the object (or objects) under investigation are defined, and in step II the region is discretized into smaller volume elements such as hexahedrons, or tetrahedrons. Here, a two-dimensional solution space has been divided into triangular elements for simplicity. The collection of segmented elements forms what is known as the mesh. In III the electric potential in a single element is approximated by a polynomial solution of the form found in equation 5.1,

$$
V_{i}(x, y)=a+b x+c y
$$

where $\mathrm{a}, \mathrm{b}$, and $\mathrm{c}$ are constants. When solving for the solutions of a single element, the electric potential is assumed to be non-zero inside the element and zero everywhere else. Moreover, the electric potential is assumed to vary linearly, such that the electric field, shown in equation 5.2, is uniform within the element.

$$
\boldsymbol{E}_{i}=-\nabla V
$$

The energy per unit length, which in this example is the desired quantity, of the element is found by equation 5.3,

$$
W_{i}=\frac{1}{2} \int \varepsilon\left|\boldsymbol{E}_{i}\right|^{2} d s
$$

where $\varepsilon$ is the permittivity within the element. After finding a solution for each element, step $\mathbf{I V}$ is to solve for the entire solution space by summing the constituent solutions. For an excellent rigorous explanation and mathematical derivation of the finite element method, the reader is referred to [12]. 
The high frequency structure simulator available from ANSYS, Inc. (ANSYS $H F S S$ ) is a full 3-dimensional frequency domain solver for Maxwell's equations [13-14]. Similar to programs such as COMSOL Multiphysics and CST Microwave Studio, ANSYS HFSS is an integrated computer aided design (CAD)-solver environment with a built in graphical user interface in which the user has the ability to draw, model, and simulate nearly any electromagnetic problem imaginable [15]. ANSYS HFSS uses the FEM, where solutions for the electric field take on the form of equation 5.4,

$$
\nabla \times\left(\frac{1}{\mu_{r}} \nabla \times \boldsymbol{E}\right)-k_{0}^{2} \varepsilon_{r} \boldsymbol{E}=\boldsymbol{J}_{\text {source }}
$$

where $\mu_{\mathrm{r}}$ is the relative permeability, $\varepsilon_{r}$ is the relative permittivity, $\mathrm{k}_{0}$ is the wave number defined as $\omega / c, \omega$ is the angular frequency of the wave, $\mathrm{c}$ is the speed of light in vacuum, and $\boldsymbol{J}_{\text {source }}$ represents the source current density. The magnetic field is calculated using equation 5.5,

$$
\boldsymbol{H}=\frac{1}{\omega \mu} \nabla \times \boldsymbol{E}
$$

and the other electromagnetic properties are derived from equations $3.5-3.7$ in Chapter 3. ANSYS HFSS finds solutions using a similar approach to that defined in figure 43. However, ANSYS HFSS uses a proprietary algorithm in which the approximated solution is then evaluated against pre-defined error criteria, and the mesh is refined into smaller elements to iterate the process until the solution falls below the error threshold [14]. This adaptive mesh process is depicted in figure 44, where between steps $\mathbf{I V}$ and $\mathbf{V}$ the difference between S-parameter solutions, defined shortly, of two consecutive iterations are compared to the preset error criterion. 


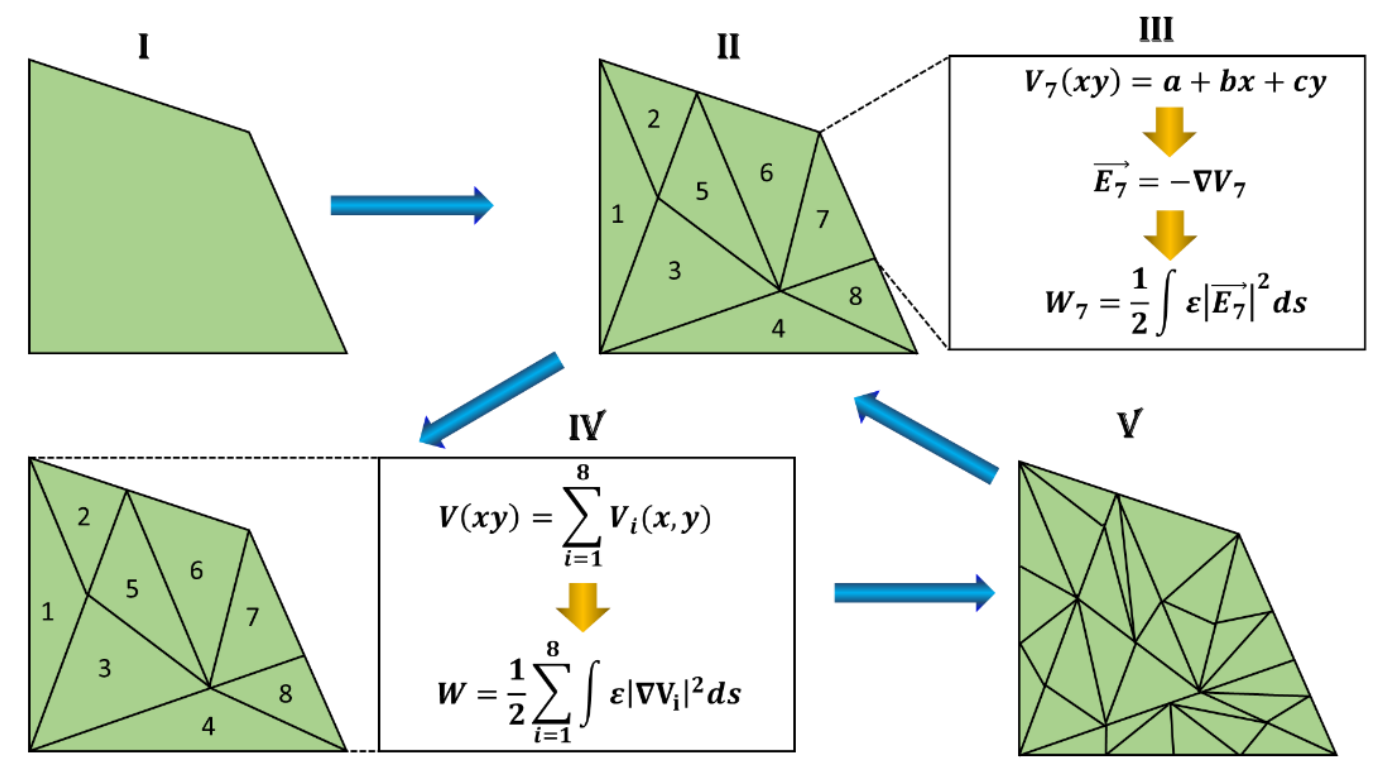

Figure 44. Simple depiction of adaptive mesh process in ANSYS HFSS.

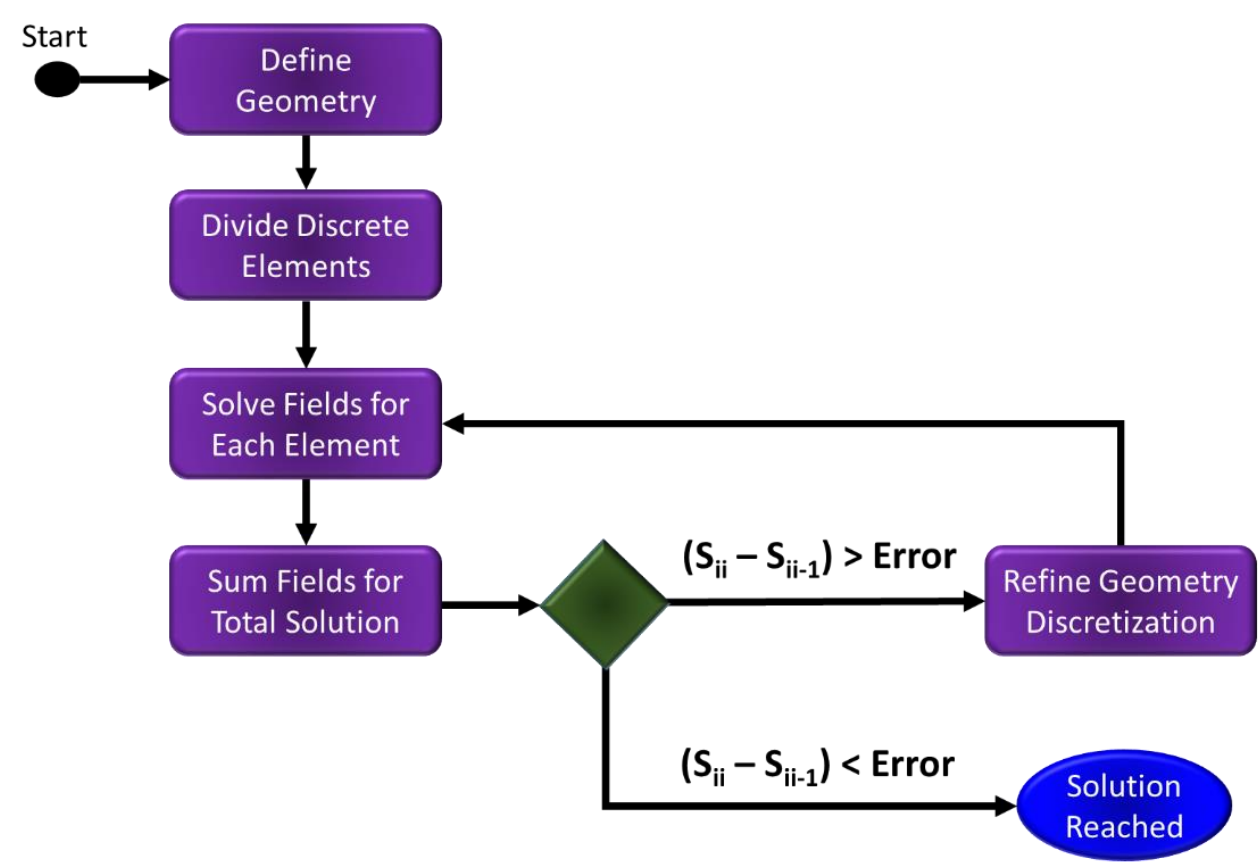

Figure 45. Simple flow block diagram for the FEM and adaptive mesh refinement in ANSYS HFSS.

For clarity, a flow block diagram of the adaptive mesh procedure is shown in figure 45 . In the next section, the details of setting up the ANSYS HFSS modeling environment will be discussed in the context of the PbSe films we investigated. 


\subsection{Modeling the PbSe Film with ANSYS HFSS}

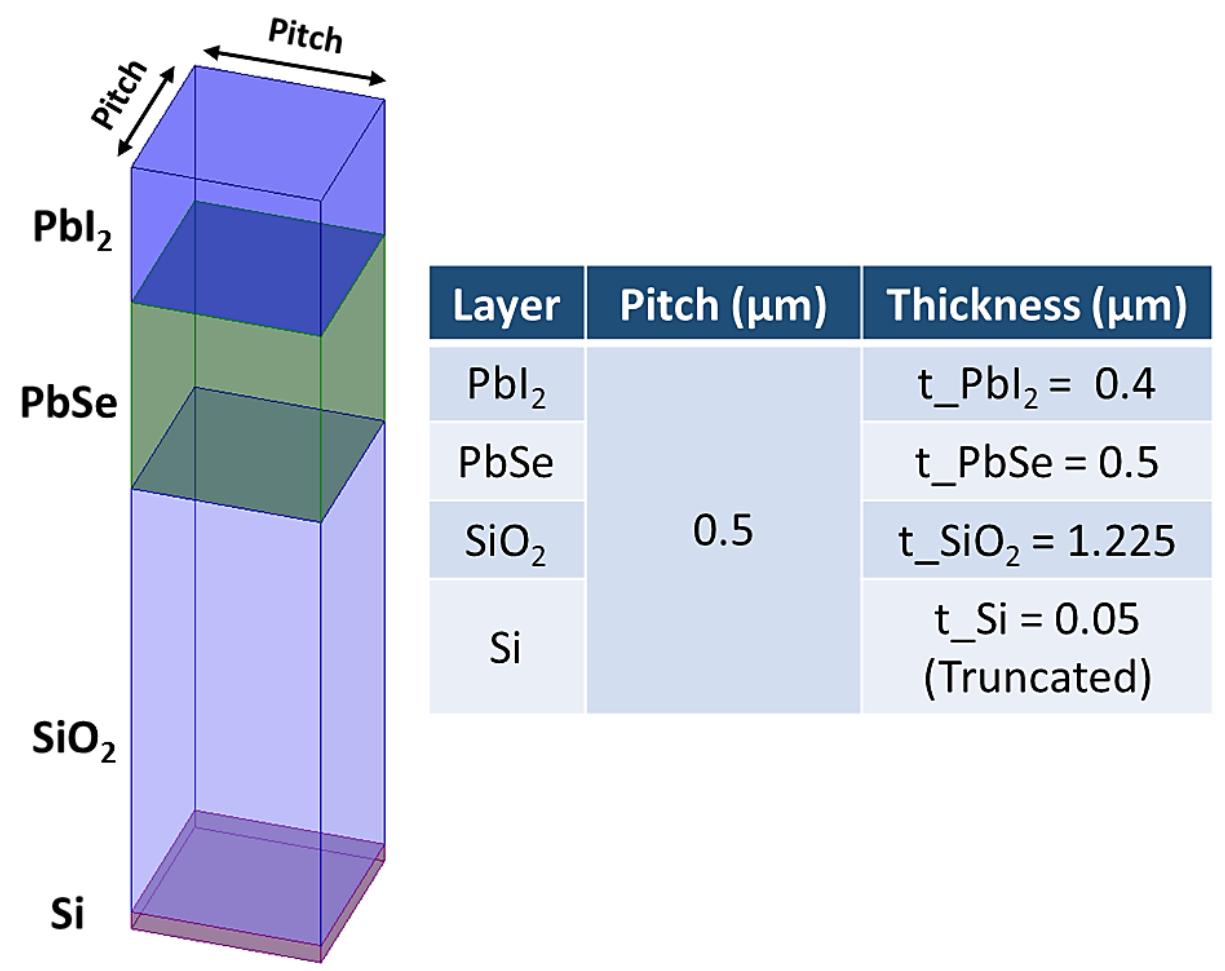

Figure 46. Unit cell of the PbSe films under investigation modeled in ANSYS HFSS.

The PbSe films we investigated, shown in figure 46, were drawn in the GUI of ANSYS HFSS. A driven modal solution setup was selected to solve for the whole film [14]. The film was input layer by layer, with the side length of each layer being input as the variable pitch. The thicknesses of each layer are input as $t_{-}$"layer/structure”, where the "layer/structure" section is replaced by the material of the layer or the structure. For example, the thickness of the PbSe layer is input as $t_{-} P b S e$. Variable names were used to enable the use of parametric analysis in the "Optimetrics" feature of ANSYS HFSS [4]. It should be noted that due to the large thickness of the Si layer $(450 \mu \mathrm{m})$, it was input with a thickness of only $50 \mathrm{~nm}$. This approximation was used to save computational time. Further, when the full substrate thickness was input, I observed several erroneous peaks 
in the modeled result. Due to the principles described in Chapter 1 and Chapter 4, it was only necessary to model the $\mathrm{SiO}_{2}-\mathrm{Si}$ interface to observe interference effects. Therefore, the truncation of the substrate was found to be an acceptable approximation.

After defining the geometry, the next step was to specify the material parameters for each layer. In ANSYS HFSS, materials are defined by two properties [8]. The first is the real part of the relative permittivity of the material, and the second is either the loss $\operatorname{tangent} \tan \delta$ or the frequency dependent conductivity $\sigma_{A C}$. The real and imaginary parts of the complex permittivity have already been defined in equations 4.14 and 4.15 of Chapter 4. The loss tangent and complex conductivity were used to simulate the losses in the material, and are given by equations 5.6 and 5.7, respectively,

$$
\tan \delta \cong \frac{\varepsilon^{\prime \prime}}{\varepsilon^{\prime}}
$$

And

$$
\sigma_{A C}=2 \pi f \varepsilon^{\prime \prime} \varepsilon_{0}
$$

where $\varepsilon^{\prime}$ and $\varepsilon^{\prime \prime}$ are the real and imaginary parts of the complex permittivity, $\mathrm{f}$ is the frequency of the electromagnetic wave, and $\varepsilon_{0}$ is the permittivity of free space [16]. Typically, the loss tangent was used in this study for PbSe, while the frequency dependent conductivity was used for Au and Pt. Recall from Chapter 2 that the other semiconductor layers were treated as non-absorbing due to their large bandgaps, and to ensure the absorption in the film is representative of the PbSe active layer. Therefore in the $\mathrm{Si}, \mathrm{SiO}_{2}$, and $\mathrm{PbI}_{2}$ layers, only the real part of the relative dielectric constant was utilized. 


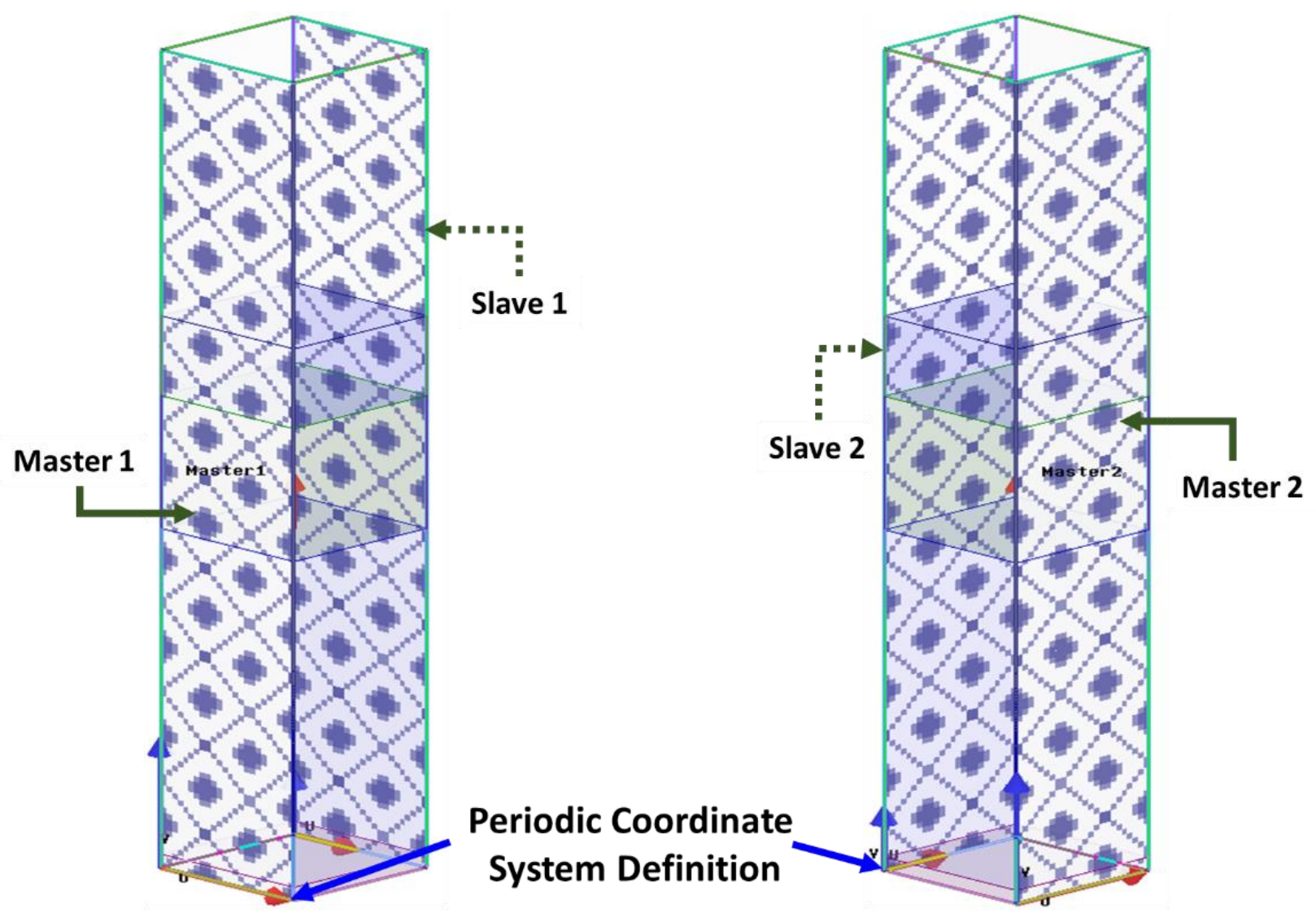

Figure 47. Periodic Master/Slave boundary conditions for modeled PbSe films.

The next step was to select the boundary conditions for the film [17]. Periodic boundary conditions, shown in figure 47, known as Master/Slave boundary conditions were assigned on the faces of the unit cell. To simplify the process of defining the boundary conditions, as well as to the ensure there was enough room between the excitation and the film surface to approximate the far field, a vacuum box $\left(\varepsilon_{r}, \mu_{r}=1\right)$ was used around the unit cell with a thickness equal to the sum of the thicknesses of each layer and an additional space of 1 micron. Master boundary conditions were placed on two adjacent faces, labeled Master1 and Master2, while Slave1 and Slave2 were placed on the opposite faces. For each master boundary condition, a coordinate system (U,V) was selected to define the direction of periodicity. With this configuration, the solutions 
of Maxwell's equations are calculated for a single unit cell, and symmetry is used at the boundaries to approximate them for either a finite or infinite array [17-18]. In the case I simulated the $\mathrm{PbSe}$ film as an infinite array to approximate the real film.

Flouquet ports, excitation ports for plane-periodic structures, were used to excite the unit cell. For these ports, the user defines a set of modes that represent plane wave modes with their direction of propagation set by the defined frequency, phase angle, and geometry of the unit cell [19]. Stated in Chapter 2 and Chapter 4, the PbSe photoconductors we investigated were designed to operate at normal incidence. Therefore, the angle of incidence in the models was kept at 0 degrees. Plane wave illumination was assumed in both the TM and TE polarization directions, so only the $\mathrm{TE}_{00}$ and $\mathrm{TM}_{00}$ modes were used. Flouquet ports offer a relatively simple way to determine the reflection, transmission, and absorption of a modeled structure in the form of S-parameters.

Scattering parameters, known as S-parameters, are defined as a transfer matrix that relates an incoming set of parameters on a multi-port system to the output of the system [20-21]. For simplicity, consider a two port system in which the input is labeled as $I_{1}$ and $\mathrm{I}_{2}$, and the output is labeled as $\mathrm{O}_{1}$ and $\mathrm{O}_{2}$. The outputs $\mathrm{O}_{1}$ and $\mathrm{O}_{2}$ are defined in terms of the inputs $I_{1}$ and $I_{2}$ by equation 5.8 .

$$
\left[\begin{array}{l}
O_{1} \\
O_{2}
\end{array}\right]=\left[\begin{array}{ll}
S_{11} & S_{12} \\
S_{21} & S_{22}
\end{array}\right]\left[\begin{array}{l}
I_{1} \\
I_{2}
\end{array}\right]
$$

Here, $S_{11}$ and $S_{22}$ are the TE and TM reflection coefficients, while $S_{12}$ and $S_{21}$ are the TE and TM transmission coefficients. Similar to the Fresnel reflection and transmission coefficients, to find the percent of the incident excitation reflected $\mathrm{R}$ or transmitted $\mathrm{T}$, the 
S-parameters must be squared. Therefore, the reflection, transmission, and absorption in the film can be found using equations 5.9-5.11.

$$
\begin{gathered}
R=S_{11}^{2} \text { or } S_{22}^{2} \\
T=S_{21}^{2} \text { or } S_{12}^{2} \\
A=1-S_{11}^{2}-S_{21}^{2} \text { or } 1-S_{12}^{2}-S_{22}^{2}
\end{gathered}
$$

After the film geometry, boundary, and excitation conditions were established, the analysis setup was defined. In ANSYS HFSS the initial solver frequency, error threshold, number of convergence passes, mesh refinement, solver type, and port impedance must be specified. Table 8 shows the analysis setup parameters I used to simulate the fields in the modeled PbSe films. When modeling resonant or frequency dependent structures, if the frequency dependent behavior is unknown the frequency should be set to the highest frequency of interest [7]. The spectral region of interest in these films is from $3 \mu \mathrm{m}$ to 5 $\mu \mathrm{m}$, so the solver frequency was set to $100000 \mathrm{GHz}$. To ensure the solver would reach convergence, the maximum number of iterative passes was set to 15 . This number of passes was chosen empirically during the modeling process as a balance between computation time, and convergence time-out. The error threshold was set to be slightly lower than the default value, again chosen empirically. The default maximum refinement, which takes advantage of the ANSYS HFSS built-in adaptive mesh algorithm, was found to be sufficient [21]. The mixed-order solver, which is the most accurate of the available solvers, was used. For periodic arrays, ANSYS HFSS has a feature called domaindecomposition in which computation time can be reduced using high performance parallel computing to distribute a model's solutions across multiple resources [22]. This feature was utilized to reduce computation time. Finally, an interpolating frequency 
sweep with 500 points was defined between $50000 \mathrm{GHz}$ and $120000 \mathrm{GHz}$. The interpolating sweep provides accurate S-parameter solutions, and is used when the fields are not necessarily the parameter of interest [14]. Later, when discussing the electric fields within the film, we use discrete frequency sweeps, in which the field quantities are saved at each frequency to calculate figures of merit.

Table 8. Specified analysis setup parameters for modeled PbSe film simulations in ANSYS HFSS.

\begin{tabular}{|l|l|l|l|l|}
\hline $\begin{array}{l}\text { Initial Solve } \\
\text { Frequency } \\
(\mathbf{G H z})\end{array}$ & $\begin{array}{l}\text { Convergence } \\
\text { Passes }\end{array}$ & $\begin{array}{l}\text { Error } \\
\text { Threshold } \\
\text { (Max Delta S) }\end{array}$ & $\begin{array}{l}\text { Maximum Mesh } \\
\text { Refinement/Pass }\end{array}$ & Solver \\
\hline 100000 & $\begin{array}{l}15 \\
\text { Minimum 3 } \\
\text { Minimum Converged } \\
\text { Passes 2 }\end{array}$ & 0.01 & $30 \%$ & $\begin{array}{l}\text { Mixed Order; } \\
\text { Domain } \\
\text { Decomposition }\end{array}$ \\
\hline
\end{tabular}

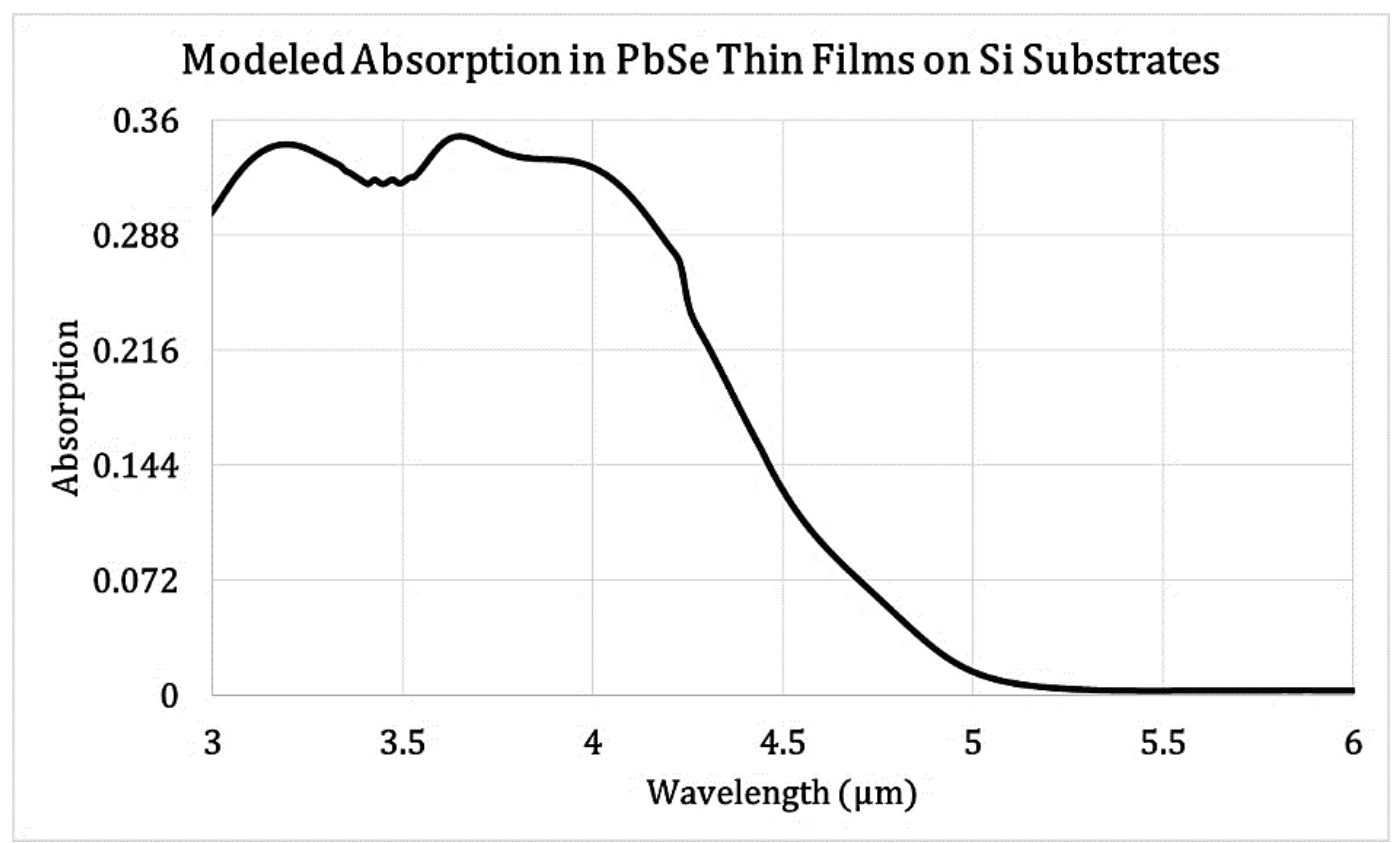

Figure 48. Modeled absorption in PbSe thin films with $1225 \mathrm{~nm} \mathrm{SiO}$, $500 \mathrm{~nm} \mathrm{PbSe,} \mathrm{and} 400 \mathrm{~nm} \mathrm{PbI}$.

To determine the absorption of the PbSe film depicted in figure 46, the $\mathrm{S}$ parameters of the TM mode were used. It should be noted that this unit cell has a polarization independent configuration, so the TE mode yielded the same results. A 
parametric analysis was conducted to determine which $\mathrm{SiO}_{2}, \mathrm{PbSe}$, and $\mathrm{PbI}_{2}$ thicknesses resulted in an absorption spectra in agreement with figure 20. It was found that $\mathrm{SiO}_{2}$ thickness of $1225 \mathrm{~nm}, \mathrm{PbSe}$ thickness of $500 \mathrm{~nm}$, and a $\mathrm{PbI}_{2}$ thickness of $400 \mathrm{~nm}$ resulted in a closely matched absorption spectrum. Figure 48 shows the absorption of the modeled film. The decrease in absorption centered at $3.5 \mu \mathrm{m}$ is associated with interference in the films, which is also present in figure 20. Moreover, this interference was also found to be present in the spectral response and quantum efficiency of the baseline detectors shown in figure 22, suggesting it even plays a significant role in our baseline PbSe detectors.

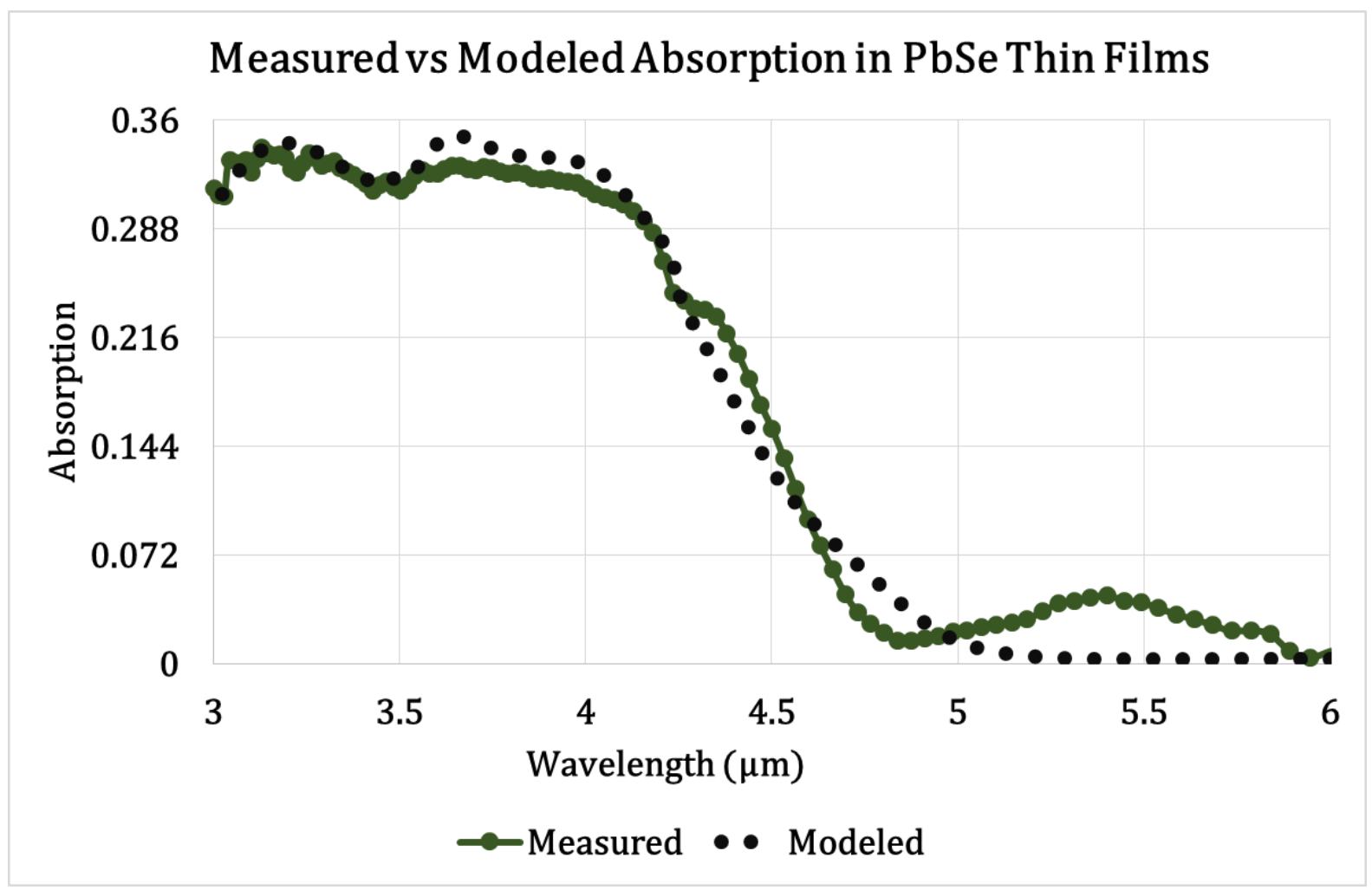

Figure 49. Comparison of modeled to measured absorption in PbSe films for model validation.

Before moving on, it is important to review how the model was validated. Modeling and simulation are powerful tools used to predict real system behavior, thereby making the engineering process more efficient. Further, they provide insight into the 
underlying mechanisms and working principles in the system under investigation. To this end, I compared the modeled and measured absorption to validate the model. Figure 49 shows that the modeled spectrum agrees well with the measured spectrum, with only minor deviations attributed to surface roughness and impurities as mentioned in Chapter 2. The close agreement between modeled and measured results shows that the model can be used to predict, at least to first order, the absorptive behavior of the investigated $\mathrm{PbSe}$ films.

\subsection{Predicted Results for Metal Discs in PbSe Films}

For surface plasmon resonant structures, the disc shape was chosen for its polarization independence and simplicity of fabrication. To select the aspect ratio, defined as the quotient of disc diameter and thickness, I completed a parametric study using a quasi-static model developed in MATLAB (see Appendix B). The Au and Pt discs were modeled with a fixed disc thickness of $20 \mathrm{~nm}$, and a disc diameter varying from 40 $\mathrm{nm}$ to $500 \mathrm{~nm}$. This resulted in a disc aspect ratio between 2 and 25. In anticipation of the limitations of fabrication on rough surfaces, the discs were considered at the $\mathrm{SiO}_{2}-\mathrm{PbSe}$ interface. The discs would be patterned and fabricated on the $\mathrm{SiO}_{2}$ before roughening and PbSe deposition. Figure 50 shows an example result from the parametric study. Recall from Chapter 3 that the extinction Q-factor is the sum of the absorption and scattering Qfactors. As seen in figure 50, the absorption Q-factor is very small, and the scattering and extinction Q-factors lie on top of one another. Therefore, the extinction Q-factor is dominated by scattering. As mentioned Chapter 3, this means the evanescent waves generated due to surface plasmon polaritons will be traveling waves. The resonance for Pt discs with a 12.5 aspect ratio was found to be at $3.74 \mu \mathrm{m}$. 


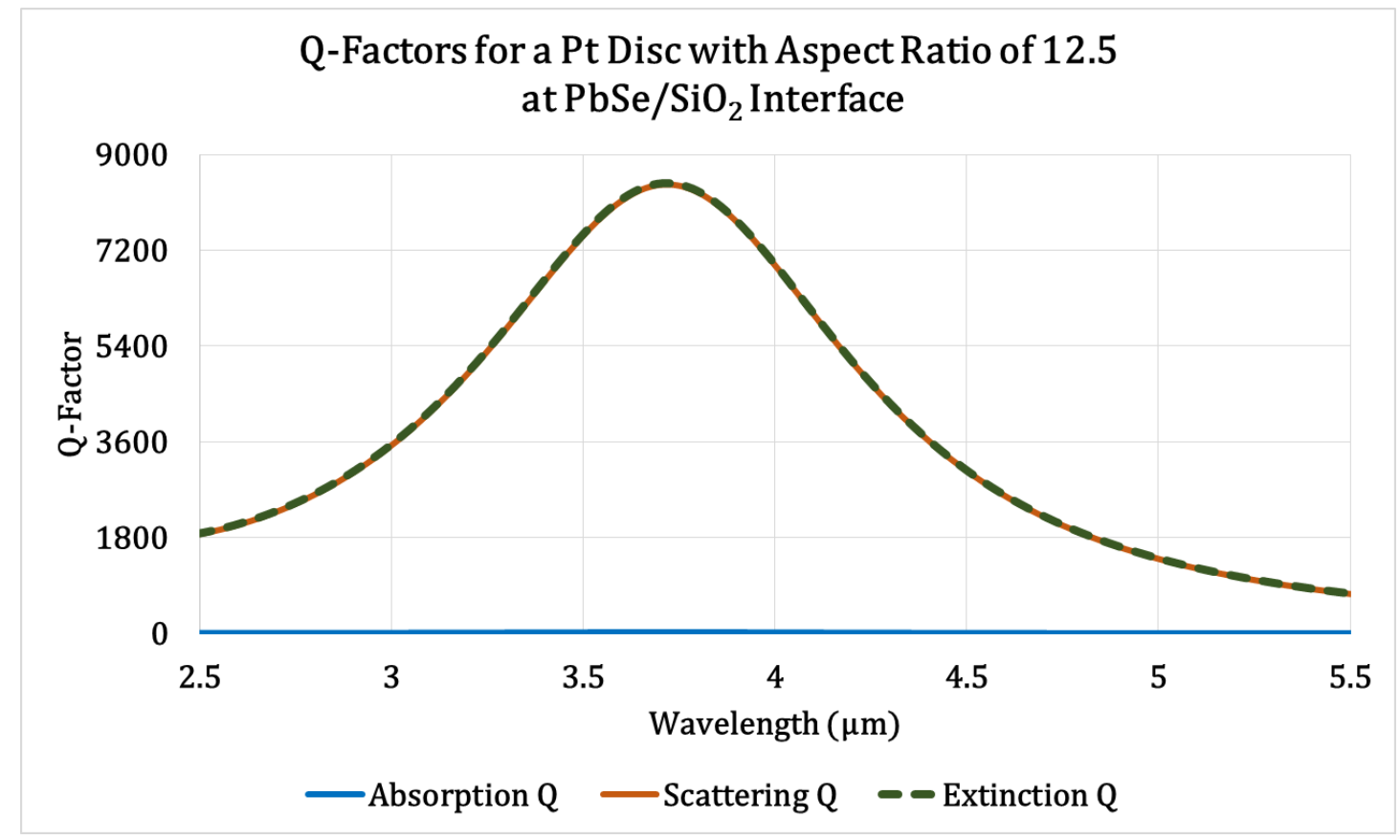

Figure 50. Efficiency $(Q)$ factors of Pt discs at $\mathrm{SiO}_{2} / \mathrm{PbSe}$ interface for an aspect ratio of 12.5 .

In both $\mathrm{Au}$ and $\mathrm{Pt}$, scattering was found to be the dominant surface plasmon phenomenon. In fact, as a general observation, scattering was found to dominate in the plasmonic disc structure regardless of metal. From Mie theory, scattering becomes the dominant mechanism for large structures [24-25]. Therefore, due to the large diameter of the discs, this observation is expected behavior and an excellent reason to choose the disc shape for enhancing PbSe detectors. Figure 51 shows how the efficiency factors for $\mathrm{Au}$ and Pt discs vary as a function of disc diameter. For larger aspect ratio discs, the resonance red-shifts, and the amplitude increases. Moreover, the width of the resonance peak, defined as the full width at half maximum, increases. For the Au discs, it increases from an estimated $0.5 \mu \mathrm{m}$ at an aspect ratio of 15 to $0.8 \mu \mathrm{m}$ at an aspect ratio of 25 . The Pt discs starts with and maintains a larger resonance peak width than the $\mathrm{Au}$; where an aspect ratio of 12.5 resulted in a width of $1.2 \mu \mathrm{m}$, and at an aspect of 25 a width of $2 \mu \mathrm{m}$. 


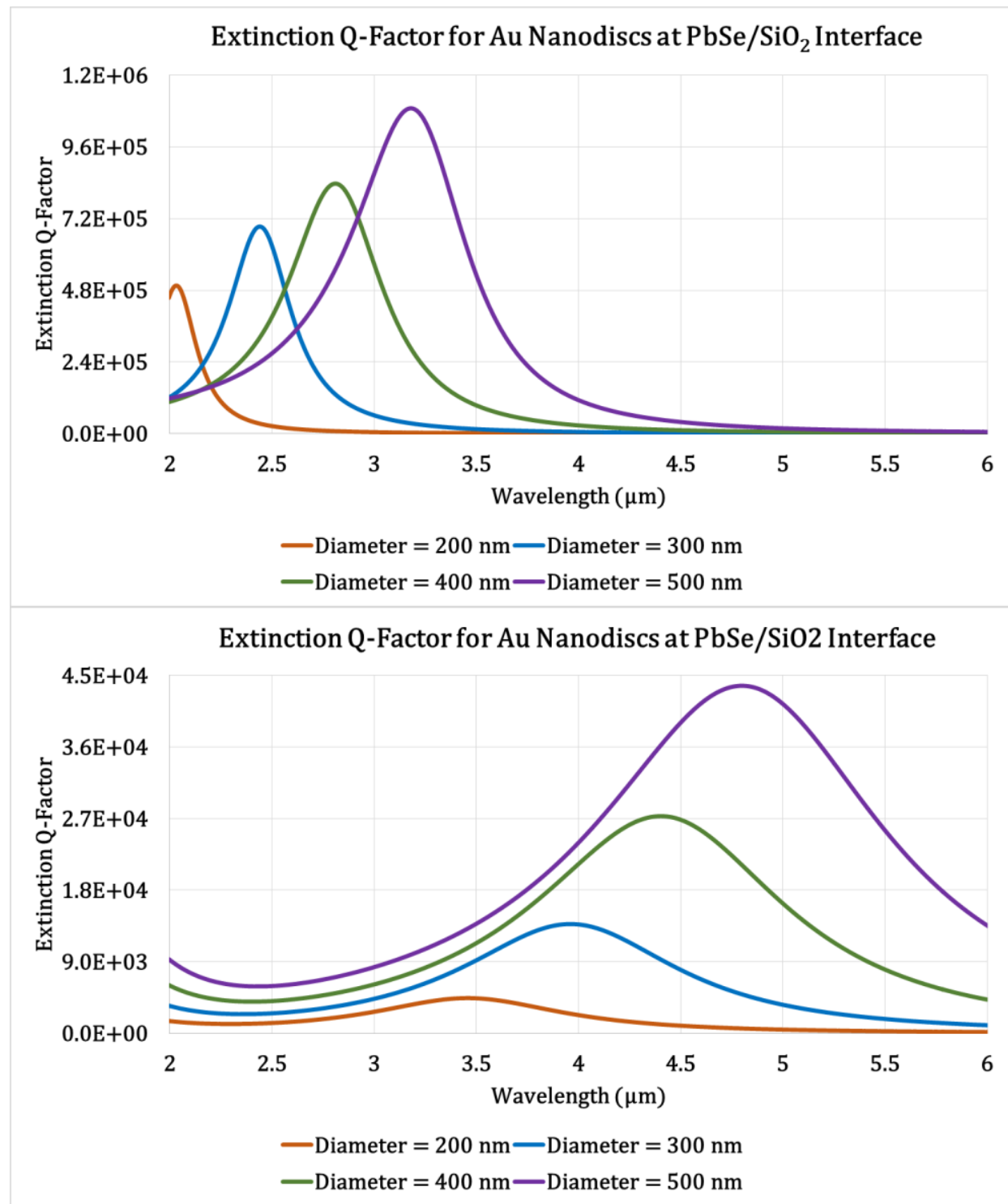

Figure 51. Results of the parametric analysis of the Au and Pt discs embedded at the $\mathrm{SiO}_{2}-\mathrm{PbSe}$ interface.

Recall from Chapter 3 that the width of the resonance peak is related to the surface plasmon lifetime, where the longer the lifetime the sharper the resonance peak [23]. When comparing the surface plasmon lifetimes of Au and Pt from figure 36, with respect to the resonance peak width, the results of figure 51 are to be expected. The goal of this investigation was to enhance $\mathrm{PbSe}$ performance over the MWIR regime. Therefore, a tradeoff exists between the narrower peaks of $\mathrm{Au}$, which offer an increased extinction efficiency (Q) factor, and the broad peaks of Pt that offer a broader 
enhancement. For this study, Pt was selected for device fabrication, in part, due to its broad resonance.

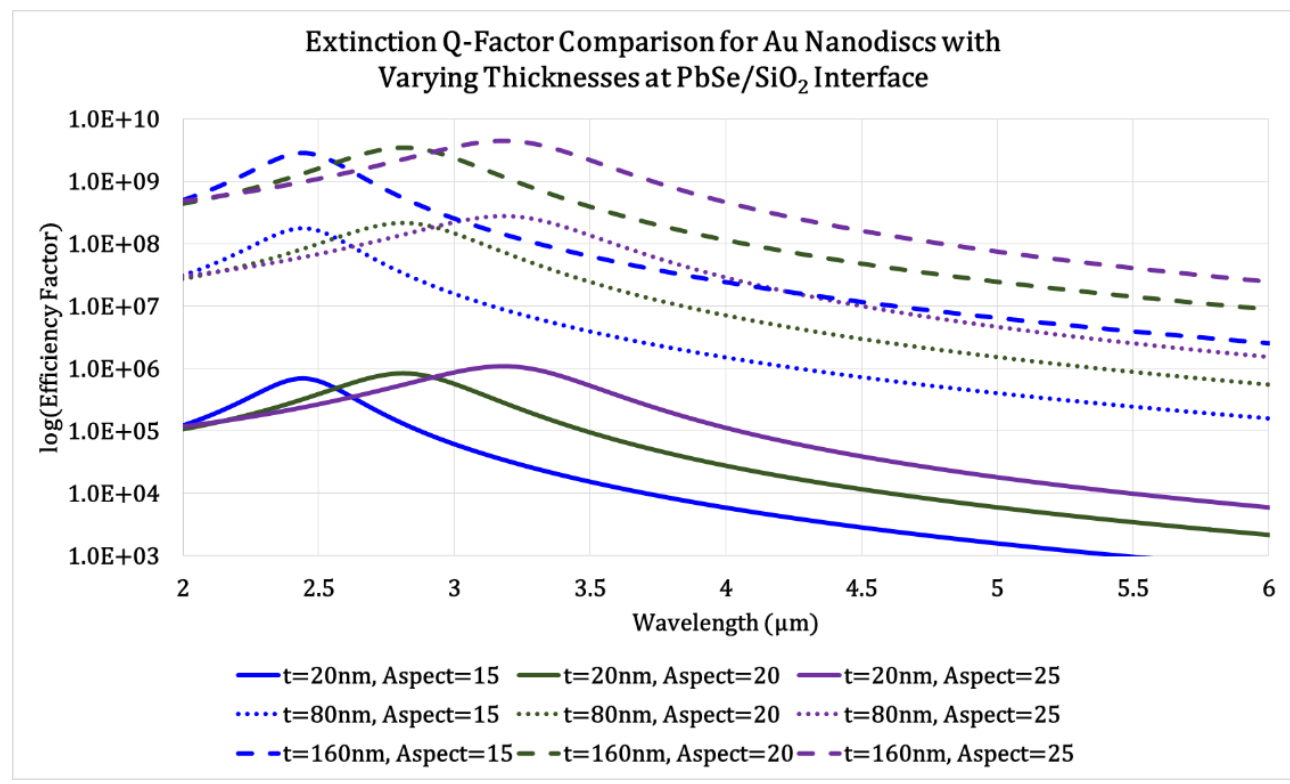

Figure 52. Comparison of $20 \mathrm{~nm}, 80 \mathrm{~nm}$, and $160 \mathrm{~nm}$ thick Au discs with fixed aspect ratios.

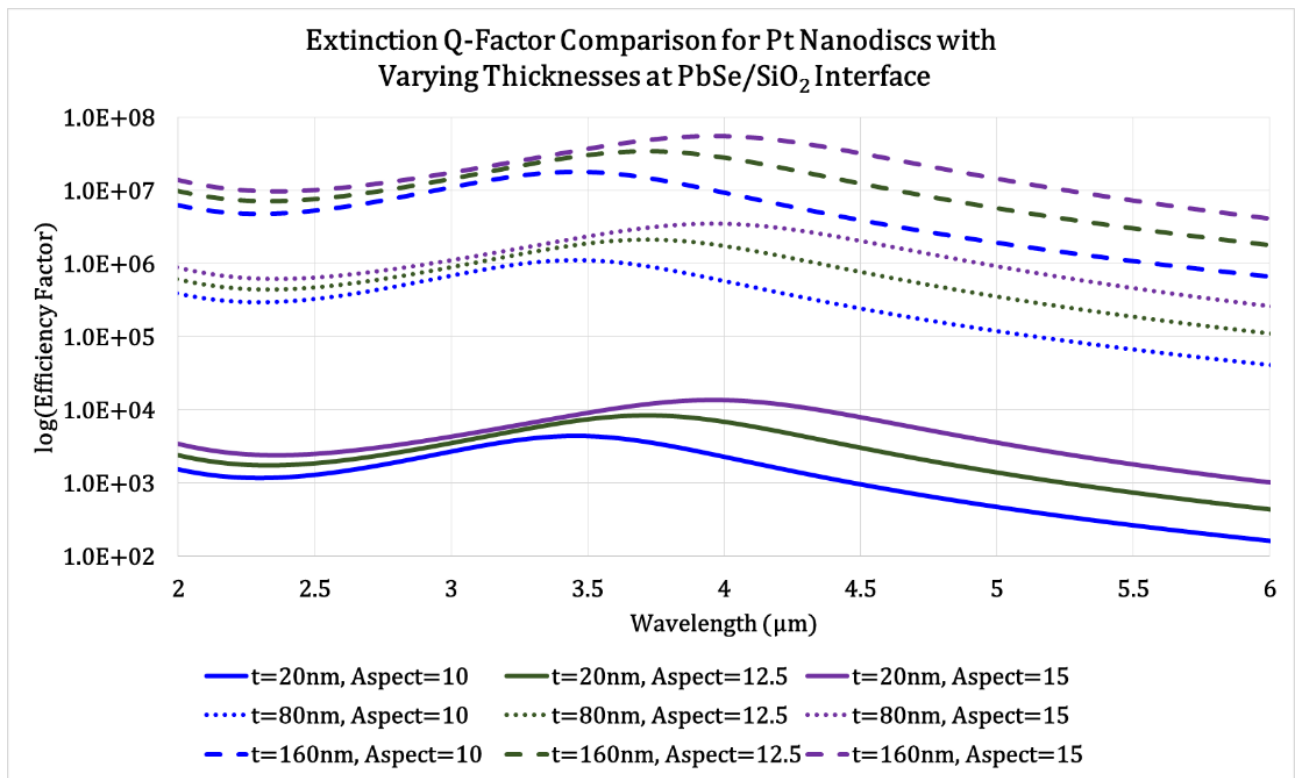

Figure 53. Comparison of $20 \mathrm{~nm}, 80 \mathrm{~nm}$, and $160 \mathrm{~nm}$ thick Pt discs with fixed aspect ratios.

To ensure the resonance peak and majority of the peak width would reside within the spectral response region of $\mathrm{PbSe}$, which has an absorption edge of $\sim 4.3 \mu \mathrm{m}$, and to 
ensure the resonance peak did not fall within the atmospheric $\mathrm{CO}_{2}$ absorption notch of $4.1 \mu \mathrm{m}-4.4 \mu \mathrm{m}$, the disc parameters were chosen such that their resonance peaks would be located between $3.5 \mu \mathrm{m}$ and $4 \mu \mathrm{m}$. For Pt, this lead to an aspect ratio of 12.5 , which has a resonance of $3.74 \mu \mathrm{m}$. However, for Au the resonance peak did not reach this region during the parametric study. It was required to extend the aspect ratio of $\mathrm{Au}$ to 30 to reach a resonance wavelength of $3.51 \mu \mathrm{m}$.

Though the disc diameters shown in figure 51 have a maximum diameter of 500 $\mathrm{nm}$, larger discs would be used in the real films due to fabrication constraints. To keep the aspect ratio fixed, larger disc diameters necessitate larger thicknesses. To this end, I modeled larger discs to observe the effect of disc diameter on the resonant behavior of $\mathrm{Au}$ and Pt discs. Keeping the aspect ratio fixed by scaling the disc diameter, disc thicknesses of $20 \mathrm{~nm}, 80 \mathrm{~nm}$, and $160 \mathrm{~nm}$ were modeled. Figures 52 and 53 demonstrate that increasing the disc thickness for the same aspect ratio does not affect the resonance. This agrees well with the assertion in Chapter 3 that the aspect ratio is the governing parameter for surface plasmon resonance for the disc structure [23]. In-turn, the larger disc diameter necessary to maintain the aspect ratio leads to an increased extinction Qfactor. Therefore, discs of up to $1 \mu \mathrm{m}$ diameter were considered feasible for fabrication. The selected parameters for finite element modeling are shown in table 9.

Table 9. Selected parameters for finite element method modeling of single Au and Pt discs.

\begin{tabular}{|c|c|c|c|}
\hline Metal & Aspect Ratio & Diameter & Resonant Wavelength \\
\hline $\mathrm{Au}$ & 30 & $\leq 1 \mu \mathrm{m}$ & $3.51 \mu \mathrm{m}$ \\
\hline $\mathrm{Pt}$ & 12.5 & $\leq 1 \mu \mathrm{m}$ & $3.74 \mu \mathrm{m}$ \\
\hline
\end{tabular}




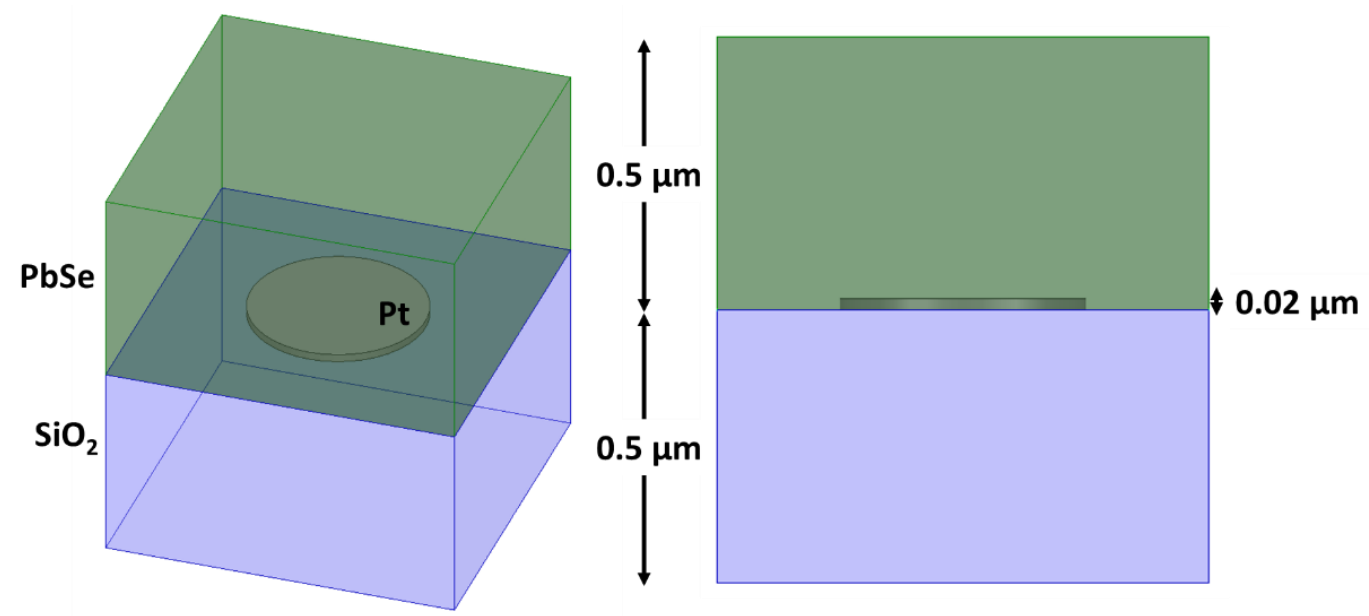

Figure 54. PbSe-SiO ${ }_{2}$ interface unit cell with single Pt disc modeled in ANSYS HFSS.

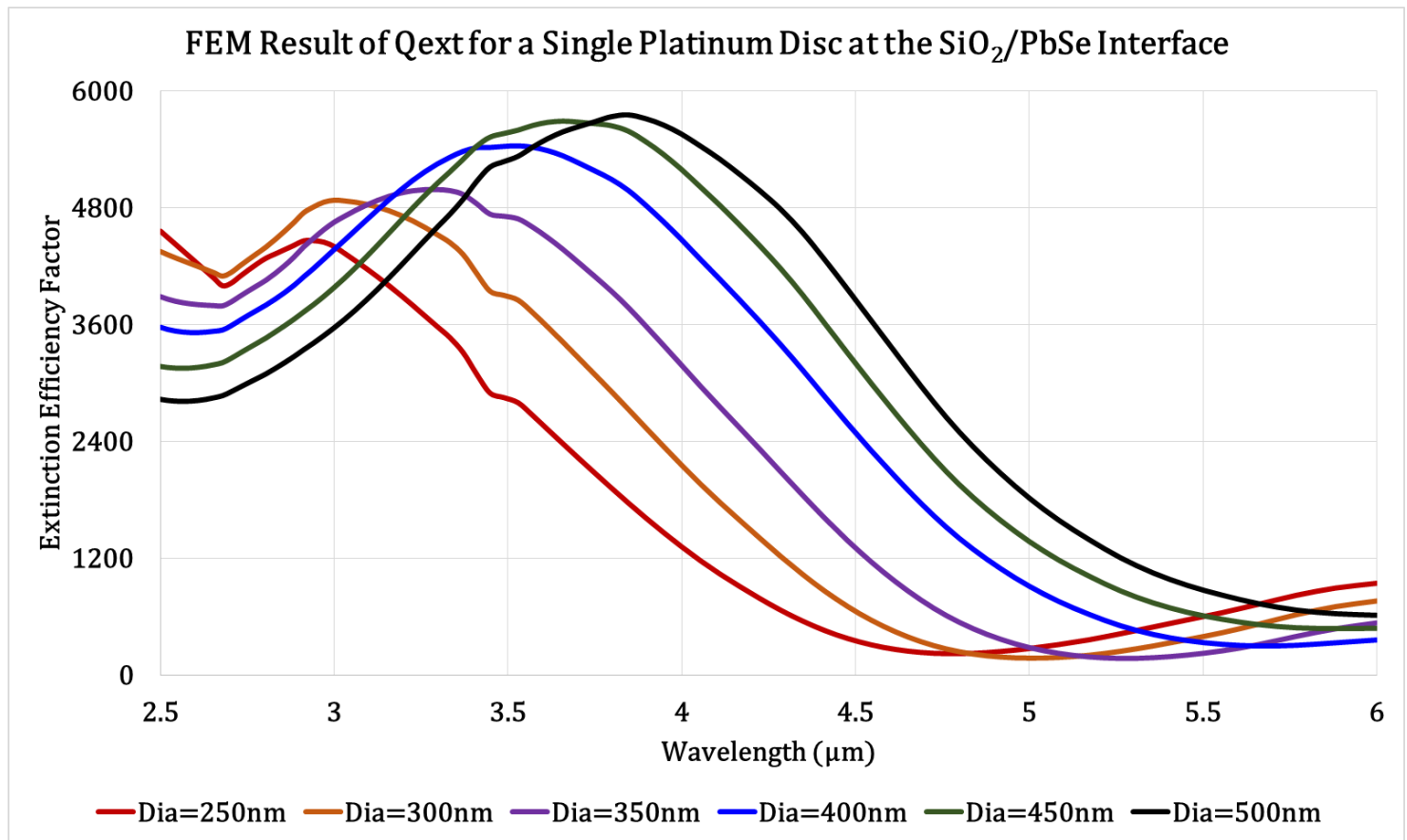

Figure 55. Extinction efficiency $(Q)$ factor, dominated by scattering, of Pt discs at the $\mathrm{SiO}_{2}$-PbSe interface modeled in ANSYS HFSS.

The aspect ratios of table 9 were found using the quasi-static model for surface plasmon resonant discs. During the parametric analysis described above, the dielectric constant of the host material, in this case the interface between the $\mathrm{SiO}_{2}$ and $\mathrm{PbSe}$, was considered constant. For higher fidelity results, a single disc at the same interface was modeled with the full dispersion relations in ANSYS HFSS. For this simulation, only a 
single unit cell was modeled to observe the fields and the Q-factors of the disc structure. Moreover, to minimize interference effects in the film, only the $\mathrm{PbSe}$ and $\mathrm{SiO}_{2}$ layer was considered. The discs in the context of the whole film will be discussed shortly.

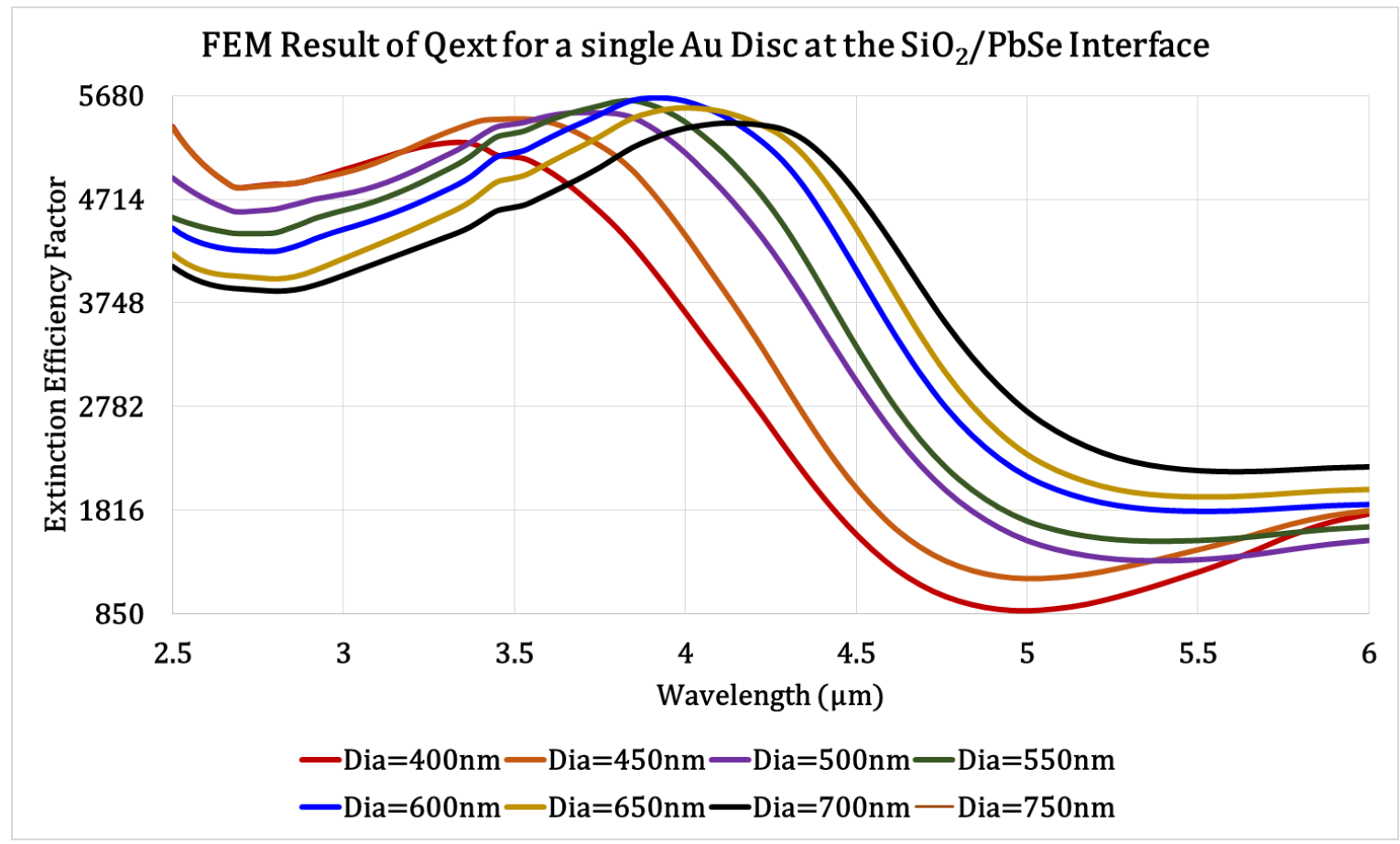

Figure 56. Extinction efficiency $(Q)$ factor, dominated by scattering, of Au discs at the $\mathrm{SiO}_{2}$-PbSe interface modeled in ANSYS HFSS.

Shown in figure 54, the layer thickness of both $\mathrm{SiO}_{2}$ and $\mathrm{PbSe}$ were set to $500 \mathrm{~nm}$. Here, radiation boundary conditions, which are absorbing boundary conditions, were used at the edge of the unit cell [17]. Excitation was in the form of an x-polarized plane wave traveling in the $-\mathrm{z}$-direction, and starting $1 \mu \mathrm{m}$ above the film. The disc radius was set to be a quarter of the unit cell pitch, such that the disc diameter was half the pitch. The disc was positioned in the center of the unit cell. A discrete frequency sweep was used to determine and utilize all of the field solutions necessary for calculating the field intensity enhancement and efficiency factors. The scattering and absorption efficiency factors were 
calculated using equation 5.12 and equation 5.13, and the extinction efficiency factor is given as their sum,

$$
\begin{gathered}
Q_{a b s}=\frac{\sigma}{\pi r_{d i s c}^{2} c \varepsilon_{0} n_{0}} \iiint_{\text {Disc Volume }}|E|^{2} d V \\
Q_{s c t}=\frac{2}{\pi r_{d i s c}^{2} c \varepsilon_{0} n_{0}} \iint_{\text {Disc Surface }}\left|E \times H^{*}\right| d S
\end{gathered}
$$

where $\frac{c \varepsilon_{0} n_{0}}{2}$ is the intensity of the incident wave, $\sigma$ is the electrical conductivity of the medium, and $\pi r_{\text {disc }}^{2}$ is the geometric cross-section of the disc transverse to the direction of illumination [7, 23].

To observe the effects of the full dispersion relations of PbSe on the surface plasmon resonance of the discs, the pitch was varied from $500 \mathrm{~nm}$ up to $1 \mu \mathrm{m}$ for Pt discs and $500 \mathrm{~nm}$ to $1.5 \mu \mathrm{m}$ for Au. The discs, in turn, were modeled with diameters between $250 \mathrm{~nm}$ and $750 \mathrm{~nm}$, depending on the metal. Finite conductivity boundary conditions were used on the disc, which utilize the input conductivity to calculate losses within the metal. These boundary conditions were necessary, because, as described in Chapter 3, the charges inside and outside the disc contribute to the evanescent waves that result from surface plasmon resonance. Figure 55 and figure 56 show the results for the extinction efficiency (Q) factors for Pt and Au discs, respectively. From the quasi-static model, it would be expected that Pt would have a smaller amplitude and a narrower resonance than Au. However, the modeled results show that Au has the smaller amplitude and broader resonance. Further, the resonances of Pt have a more symmetrical shape, while the Au discs exhibit a more asymmetric. 


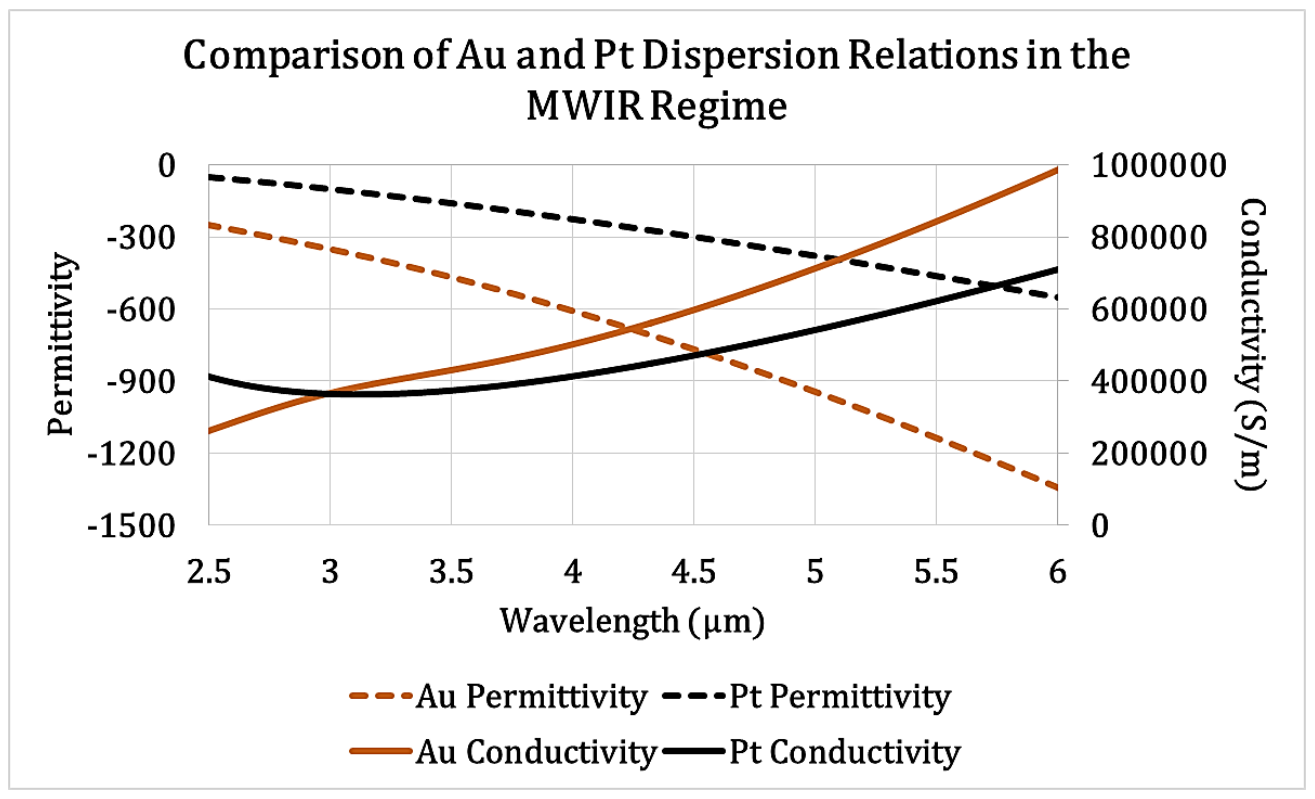

Figure 57. Comparison of $A u$ and Pt dispersion relations from $2.5 \mu \mathrm{m}$ to $6 \mu \mathrm{m}$.

Consider the quasi-static resonance condition for surface plasmons, which considers the host material to have a fixed dielectric constant. Figure 33 shows that $\mathrm{Au}$ has long surface plasmon lifetime (up to $\sim 500 \mathrm{~ns}$ at the PbSe absorption edge of $4.3 \mu \mathrm{m}$ ), leading to a narrow resonance line width. However, figure 28 shows that the dielectric constant of PbSe is highly frequency dependent in the MWIR region. Therefore, the changes in the dielectric constant of PbSe create a broad background continuum, where for each different value there is a narrow Au resonance condition. Fano resonances describe the asymmetric line shape that arises from the interference between a broad background continuum and a narrow resonance [24-26]. Therefore the asymmetry observed in figure 56 is associated with Fano resonance. Though the Pt absorption spectra is more symmetric, Fano resonances were observed in the spectra of figure 55 as well. However, due to the lower surface plasmon lifetime for Pt, the resonances are broader and so its asymmetry is less pronounced. The Fano resonances observed suggest 
that the absorption enhancement in $\mathrm{PbSe}$ will be more asymmetric than the results of figure 51 would suggest. As described in Chapter 6, I found this assertion to be true.

For both $\mathrm{Pt}$ and $\mathrm{Au}$, it would seem that for the resonance condition to reside between $3.5 \mu \mathrm{m}$ and $4 \mu \mathrm{m}$, the required diameter is between $400 \mathrm{~nm}$ to $500 \mathrm{~nm}$. This similarity is perhaps the most startling result, because it suggests that the material of the disc does not change the resonance condition dramatically as the quasi-static model would suggest. Figure 57 shows the differences between the electrical properties of $\mathrm{Au}$ and Pt. The Au has a lower permittivity and a higher conductivity than Pt. With its lower conductivity Pt has a higher absorption cross-section than $\mathrm{Au}$, which explains the observed increase in the extinction Q-factor in Pt. Recall from Chapter 3 that the permittivity is an important parameter for surface plasmon resonance. From the difference in the permittivity of $\mathrm{Au}$ and $\mathrm{Pt}$, a first assumption would be that the resonances should be different for the same aspect ratio discs. Recall that for discs, the aspect ratio is just as important for resonance. In fact, the results of figures 55 and 56 suggest aspect ratio is more important than the metal type for the resonance condition of these discs in PbSe films.

Along with the increase in extinction, it was important to determine the electric field intensity enhancement at the resonance wavelength. Indeed, the importance of the electric field intensity enhancement was emphasized in equation 3.35 in Chapter 3 while describing the carrier generation rate in photoconductors. The intensity enhancement is found by taking the ratio of the resulting wavelength-dependent electric field intensity to that of the incident intensity. The incident field intensity in these models is that of a unit 
amplitude plane wave. I calculated the electric field intensity enhancement in the xz-, yz-, and xy-planes for both $\mathrm{Au}$ and $\mathrm{Pt}$ discs at the $\mathrm{SiO}_{2} / \mathrm{PbSe}$ interface using ANSYS HFSS.

\begin{tabular}{|r|}
\hline Intensity Enhancement $(\mathbf{x z})$ \\
3819.2 \\
3604.3 \\
3389.5 \\
3174.6 \\
2959.8 \\
2744.9 \\
2530.1 \\
2315.2 \\
2100.4 \\
1885.5 \\
1670.7 \\
1455.8 \\
1241.0 \\
1026.1 \\
811.3 \\
596.4 \\
\hline
\end{tabular}
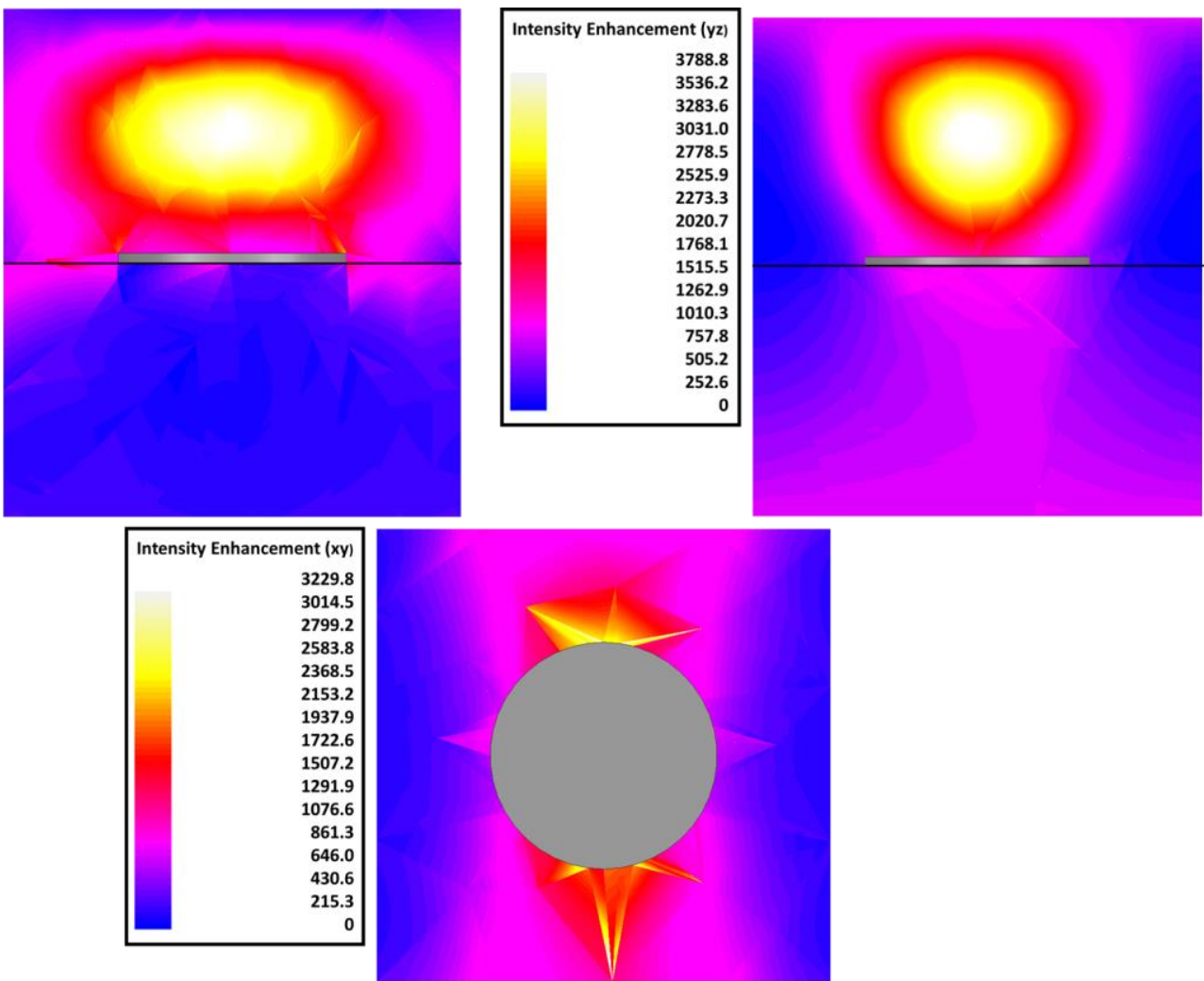

Figure 58. Modeled electric field enhancement plots in the (top-left) xz-, (top-right) yz-, and (bottom) xydirections for a single Pt disc at $\mathrm{SiO}_{2} / \mathrm{PbSe}$ interface.

Recall that the plane wave is $\mathrm{x}$-polarized and propagates in the -z-direction.

Therefore the largest intensity enhancement is observed in the xz-plane, followed by the xy-plane, and finally the yz-plane. The field intensity plots for both Au and Pt are shown in figure 58 and figure 59, respectively. Both materials produce large intensity enhancements, as seen from the xz-plane, in the middle of the PbSe film. The Au has a slightly higher maximum intensity enhancement of 3944 over Pt at 3819 , yet from figure 51 it would be expected that Au would lead to a much larger enhancement than Pt.

The field behavior varies in the yz-plane as well, with both the Au and Pt discs 
leading to an increase in field intensity in $\mathrm{PbSe}$ and throughout $\mathrm{SiO}_{2}$. This same similarity between Au and Pt discs was observed when comparing figures 55 and figure 56, reaffirming the assertion made above that aspect ratio is more important for surface plasmon resonance than the material characteristics of the metal discs. It can be asserted from these results that embedding either Au or Pt disc arrays into PbSe films would lead to similar sensitivity enhancement. Indeed, when modeling the full film structure, perfect electrical conductor boundary conditions, described below, were used, so the results were metal independent.
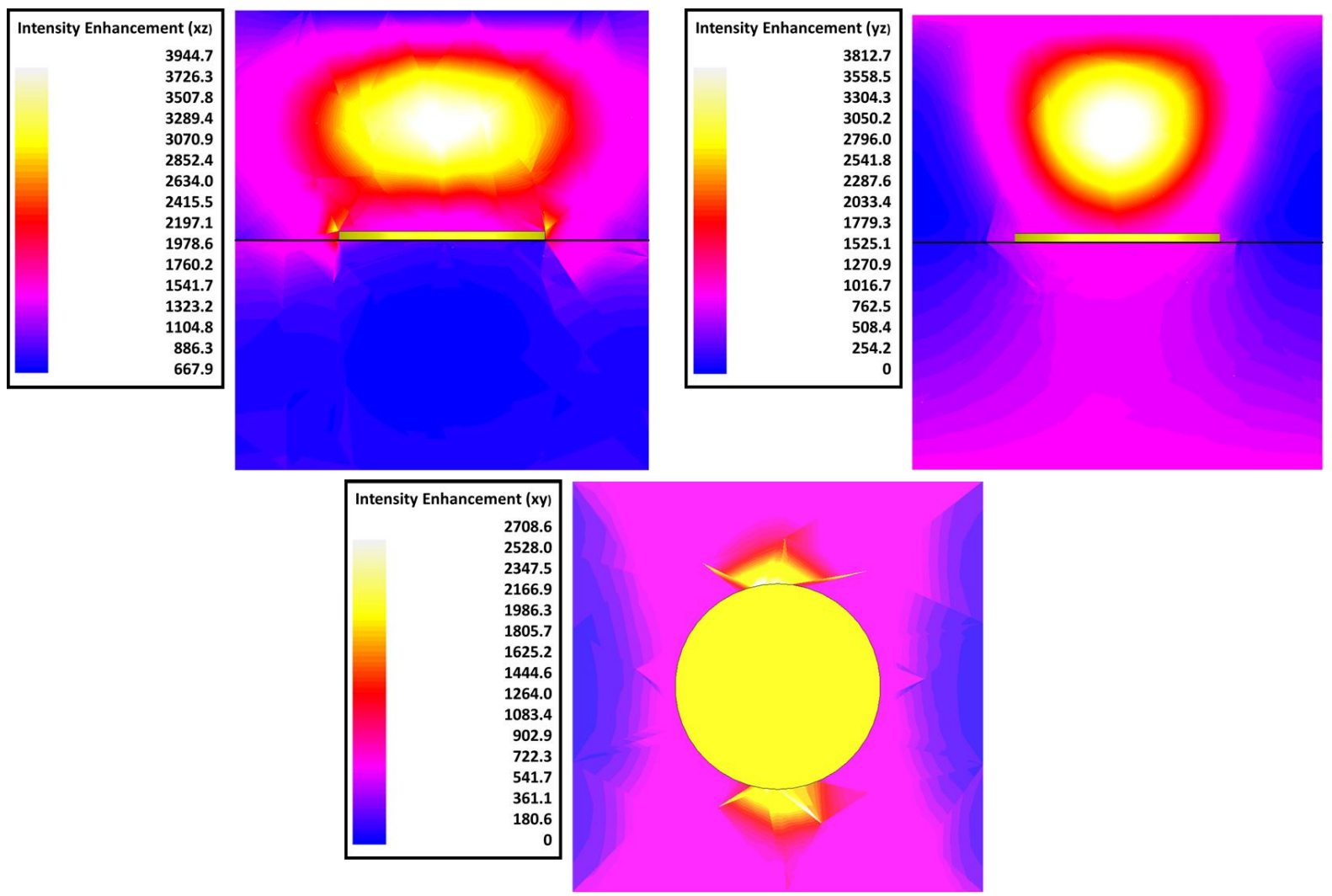

Figure 59. Modeled electric field intensity enhancement plots in the (top-left) xz-, (top-right) yz-, and (bottom) xy-directions for a single $\mathrm{Au}$ disc at $\mathrm{SiO}_{2} / \mathrm{PbSe}$ interface.

My goal in modeling the single discs was to determine the expected field enhancement in PbSe films. Though the scattering cross-section and field enhancement 
are good figures of merit for preliminary studies, the desired effect of embedding surface plasmon resonant structures into the PbSe detectors was to enhance the absorption in the investigated films. To this end, infinite arrays, with a unit cell shown in figure 60, of Pt and $\mathrm{Au}$ discs with diameters from $250 \mathrm{~nm}$ to $1000 \mathrm{~nm}$ and a fixed thickness of $40 \mathrm{~nm}$ were modeled in the full PbSe film structure in a similar manner described above. Discs were placed $30 \mathrm{~nm}$ below the $\mathrm{SiO}_{2}-\mathrm{PbSe}$ interface.

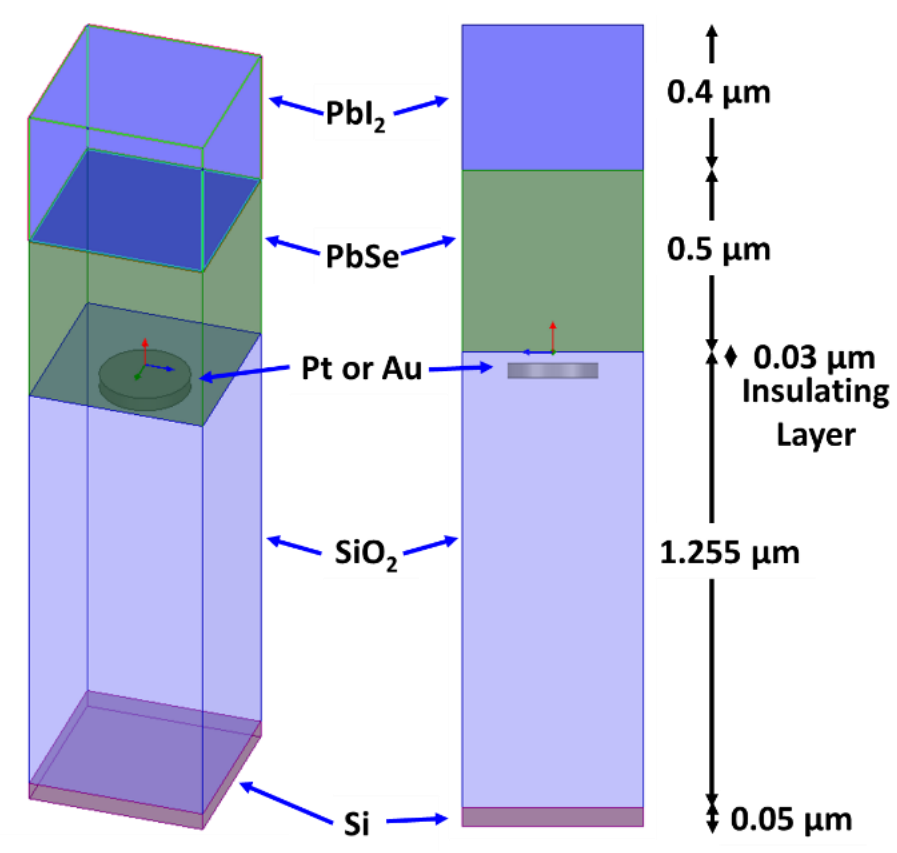

Figure 60. The modeled unit cell of the PbSe film with embedded metallic discs modeled in ANSYS HFSS.

As described in [27], quenching, a process in which surface charges generated during SPR excitation can recombine in the metal, can occur thereby negating the enhancement. Therefore, a $30 \mathrm{~nm}$ insulation layer of $\mathrm{SiO}_{2}$ was inserted on top of the disc to mitigate this potential parasitic process. The perfect electric conductor (PEC) boundary conditions were used for this simulation to separate any losses in the metal from the absorption spectra [17]. Due to this boundary condition, the spectra for both $\mathrm{Au}$ and $\mathrm{Pt}$ 
were found to be the same. However, it should be noted that for Pt discs more losses would be expected, leading to lower overall enhancement. The absorption over the range of $2.5 \mu \mathrm{m}$ to $6 \mu \mathrm{m}$ was calculated using an interpolated frequency sweep and equation 5.11 .

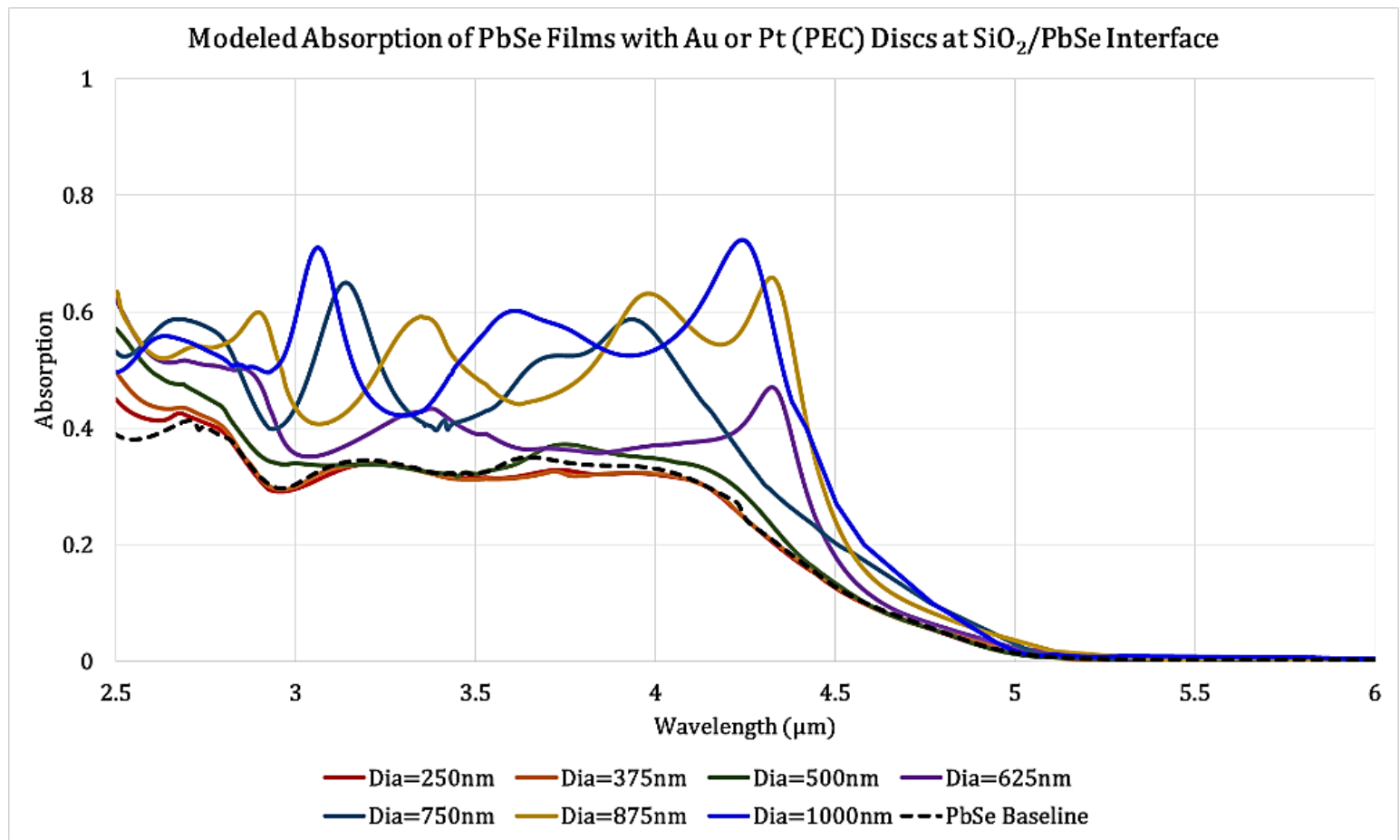

Figure 61. Modeled absorption in PbSe films with metallic (PEC) discs at $\mathrm{SiO}_{2} / \mathrm{PbSe}$ interface.

The predicted absorption for various diameters of PEC discs in PbSe films is shown in figure 61. Discs with diameters below $500 \mathrm{~nm}$ (aspect ratio smaller than 12.5) are shown to result in an absorption that is similar to the baseline PbSe absorption. Once the aspect ratio reaches 12.5 (green), there starts to be absorption enhancement. As the discs become larger the absorption continues to grow, and multiple resonance peaks develop. Up to a factor of three enhancement over the baseline PbSe absorption was predicted, depending on disc diameter and spectral region of interest. These results predict that surface plasmon resonant discs can lead to a large sensitivity increase in the 
$\mathrm{PbSe}$ films we investigated. Notice how for the $625 \mathrm{~nm}$ diameter and $875 \mathrm{~nm}$ diameter discs in figure 61 there is a resonance peak at $3.35 \mu \mathrm{m}$. This region is close to the previously observed reduction in absorption due to interference in the baseline $\mathrm{PbSe}$ films shown in figure 20 . For the $750 \mathrm{~nm}$ and $1000 \mathrm{~nm}$ diameter discs, the peak is red-shifted, and the dip in absorption is again present in the region around $3.4 \mu \mathrm{m}$. Therefore, peaks in the vicinity of this region are associated with surface plasmon resonance.

As the disc diameter increases, more peaks appear suggesting that interference starts to play a more significant role. In multilayer films and optical cavities, the separation between interference peaks can be determined by the free spectral range, which gives the wavelength separation between adjacent spectral peaks [28-30]. Using the thicknesses of the film found in figure 60, we can determine the separation between peaks due to interference using equation 5.14 ,

$$
\Delta \lambda \cong \frac{\lambda_{0}^{2}}{2 n t \cos \theta+\lambda_{0}}
$$

where $\lambda_{0}$ is the vacuum wavelength of the peak of interest, and the refractive indexthickness product $n t$ is the optical thickness of the layer. For multiple layers, the effective optical thickness is the sum of the optical thicknesses of each layer. For instance, consider the predicted absorption spectra for the $1000 \mathrm{~nm}$ disc in figure 61 . The peak centered at $3.6 \mu \mathrm{m}$ is associated with SPR, and will be ignored for now. There are two other peaks of interest in the $3 \mu \mathrm{m}-5 \mu \mathrm{m}$ region. These are centered at $3.07 \mu \mathrm{m}$ and 4.25 $\mu \mathrm{m}$, a wavelength span of $1.18 \mu \mathrm{m}$. If the free space wavelength is that of the peak at $4.25 \mu \mathrm{m}$, the free spectral range is calculated to be $1.2 \mu \mathrm{m}$, which is within $1.7 \%$ of the modeled distance between peaks. This example serves to reinforce the assertion of 
interference in the investigated films, and shows that with embedded discs the SPR and interference work together to provide a broadband absorption enhancement in the $\mathrm{PbSe}$.

\subsection{Predicted Results for an Embedded Reflector Layer in PbSe Films}

Discussed in Chapter 1 and Chapter 4, the back reflector is an attractive structure for PbSe photoconductor enhancement. Consider figure 41 and figure 42 in Chapter 4 , which show how varying the $\mathrm{SiO}_{2}$ thickness in the back reflector structure effects the absorption of PbSe. It was stated that the desired thickness of the $\mathrm{SiO}_{2}$ layer is around $1400 \mathrm{~nm}$ to yield an absorption increase that is a factor of 2.55 and 2 above the baseline $\mathrm{PbSe}$ films at $3.45 \mu \mathrm{m}$ and $4.2 \mu \mathrm{m}$, respectively. To observe the resulting field intensity within this configuration, and to verify the numerical results, it was modeled in ANSYS HFSS.

A unit cell similar to that of figure 46 was used as the geometry of these simulations. A $50 \mathrm{~nm}$ Pt layer was inserted, and given PEC boundary conditions to ensure the only absorption observed in the model would be from the PbSe layer. To further optimize the $\mathrm{SiO}_{2}$ thickness, the $\mathrm{SiO}_{2}$ layer in the ANSYS HFSS model was varied from $1400 \mathrm{~nm}$ to $1500 \mathrm{~nm}$. Due to the fact that the enhancement mechanism in these films is optical interference, the thickness of the films can affect the resonant shape dramatically. Recall that the $\mathrm{PbSe}$ deposition process involves growing a layer of $\mathrm{PbSe}$, and then converting approximately half of the layer to $\mathrm{PbI}_{2}$ for sensitization. Therefore, for these simulations the $\mathrm{PbSe}$ thickness is assumed fixed, while the $\mathrm{PbI}_{2}$ thickness was varied from $300 \mathrm{~nm}$ to $400 \mathrm{~nm}$ to account for variation in the sensitization process. I observed experimental results that supported the reduced $\mathrm{PbI}_{2}$ thickness, and were in good 
agreement with modeled predictions. Table 10 shows the parameters used for the different film thicknesses modeled.

The modeled results of figure 64 agree well with those of figure 41, which validates the predicted absorption increase in figure 42. For configurations 1 and 2 , the increase is even more pronounced. In all three configurations, the peaks of the spectrum are predicted to red-shift for $1500 \mathrm{~nm}$ thick $\mathrm{SiO}_{2}$ over that of the $1400 \mathrm{~nm}$ thick layer. For configuration 1 and 2, this red-shift leads to larger peaks at the PbSe absorption ( $\sim 4.3$ $\mu \mathrm{m})$ edge, which can be beneficial.

The detector sensitivity, specifically the spectral responsivity, is weighted by photon energy $h v$ according to equation 5.15,

$$
\mathfrak{R}(h v)=\frac{A \eta_{i} q i_{\text {photo }}}{h v P_{\text {inc }}}
$$

where $\mathrm{A}$ is the absorption of the active material, $\eta_{i}$ is the internal quantum efficiency defined as the ratio of the absorbed photons to current generating carriers, $\mathrm{q}$ is the charge of the electron, $i_{\text {photo }}$ is the magnitude of the photogenerated current, and $\mathrm{P}_{\text {inc }}$ is the power incident on the detector. This relationship shows that there should be higher response at longer wavelengths. Moreover, the photon flux is also weighted toward higher wavelengths for thermal energies close to room temperature (see Appendix C), so the $\mathrm{SiO}_{2}$ thickness should be designed such that a large enhancement is achieved at longer wavelengths. Therefore, a $1500 \mathrm{~nm} \mathrm{SiO}$ layer was selected for fabrication. A secondary peak was observed at $3.11 \mu \mathrm{m}, 3.32 \mu \mathrm{m}$, and $3.5 \mu \mathrm{m}$ for $\mathrm{PbI}_{2}$ thicknesses of $300 \mathrm{~nm}, 350 \mathrm{~nm}$, and $400 \mathrm{~nm}$, respectively. This secondary peak serves to broaden the absorption enhancement, and lead to even greater response. 
Table 10. Thickness parameters for three different configurations of PbSe films used to observe the effect of the $\mathrm{PbI}_{2}$ and $\mathrm{SiO}_{2}$ layer thickness on the absorption in PbSe films with a Pt back reflector.

\begin{tabular}{|c|c|c|}
\hline Configuration & Layer & Thickness (nm) \\
\hline \multirow{4}{*}{1} & $\mathrm{PbI}_{2}$ & 300 \\
\cline { 2 - 3 } & $\mathrm{PbSe}$ & 500 \\
\cline { 2 - 3 } & $\mathrm{SiO}_{2}$ & $1400-1500$ \\
\cline { 2 - 3 } & $\mathrm{Pt}$ & 50 \\
\cline { 2 - 3 } & $\mathrm{Si}$ & 50 \\
\hline \multirow{4}{*}{2} & $\mathrm{PbI}_{2}$ & 350 \\
\cline { 2 - 3 } & $\mathrm{PbSe}$ & 500 \\
\cline { 2 - 3 } & $\mathrm{SiO}_{2}$ & $1400-1500$ \\
\cline { 2 - 3 } & $\mathrm{Pt}$ & 50 \\
\cline { 2 - 3 } & $\mathrm{Si}$ & 50 \\
\hline \multirow{4}{*}{3} & $\mathrm{PbI}_{2}$ & 400 \\
\cline { 2 - 3 } & $\mathrm{PbSe}$ & 500 \\
\cline { 2 - 3 } & $\mathrm{SiO}_{2}$ & $1400-1500$ \\
\cline { 2 - 3 } & $\mathrm{Pt}$ & 50 \\
\cline { 2 - 3 } & $\mathrm{Si}$ & 50 \\
\hline \multirow{4}{*}{} & & \\
\hline \multirow{4}{*}{} & & \\
\hline
\end{tabular}

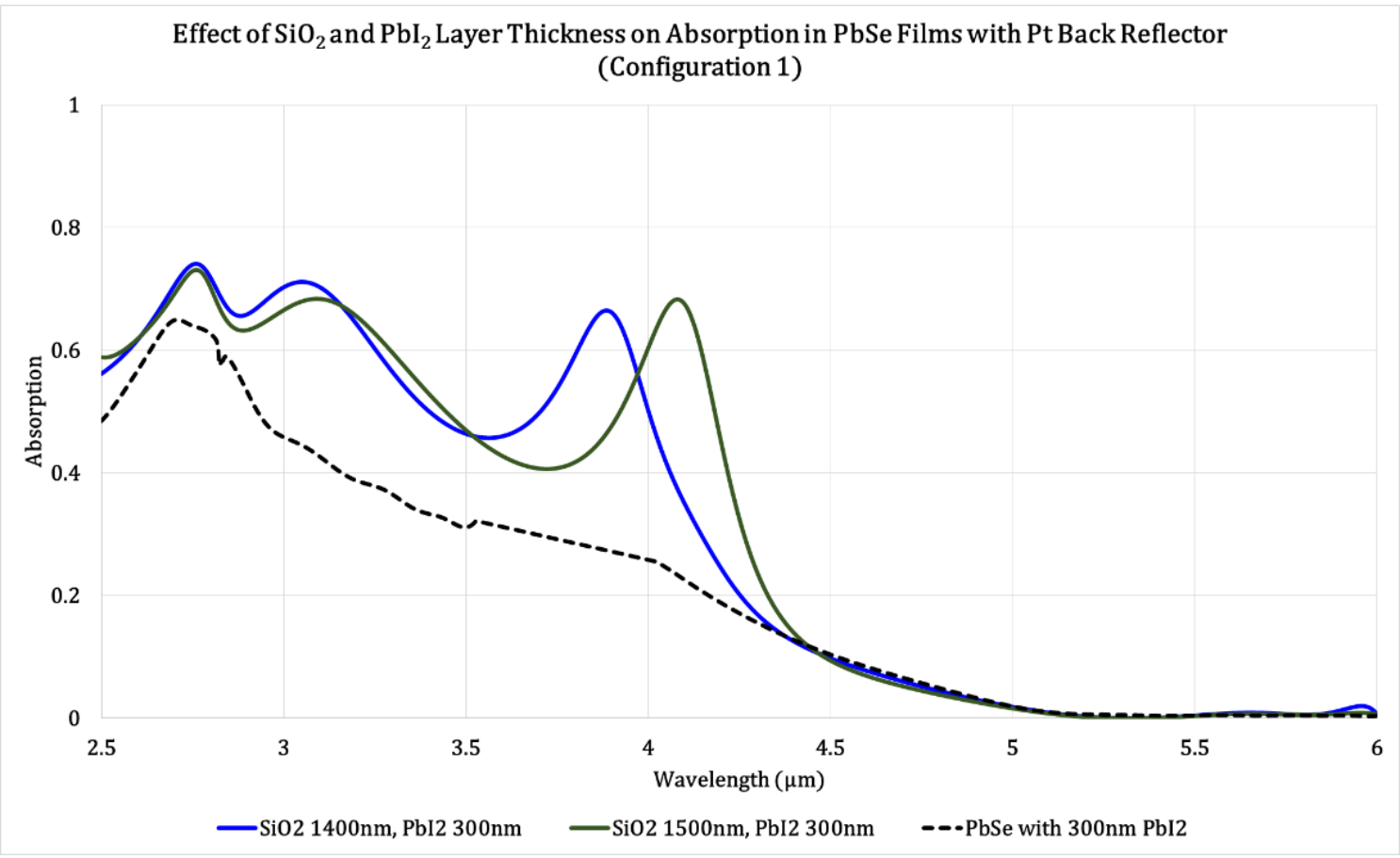

Figure 62. Effect of $\mathrm{SiO}_{2}$ and $\mathrm{PbI}_{2}$ layer thickness on absorption in PbSe Films with Pt back reflector in configuration 1 . 


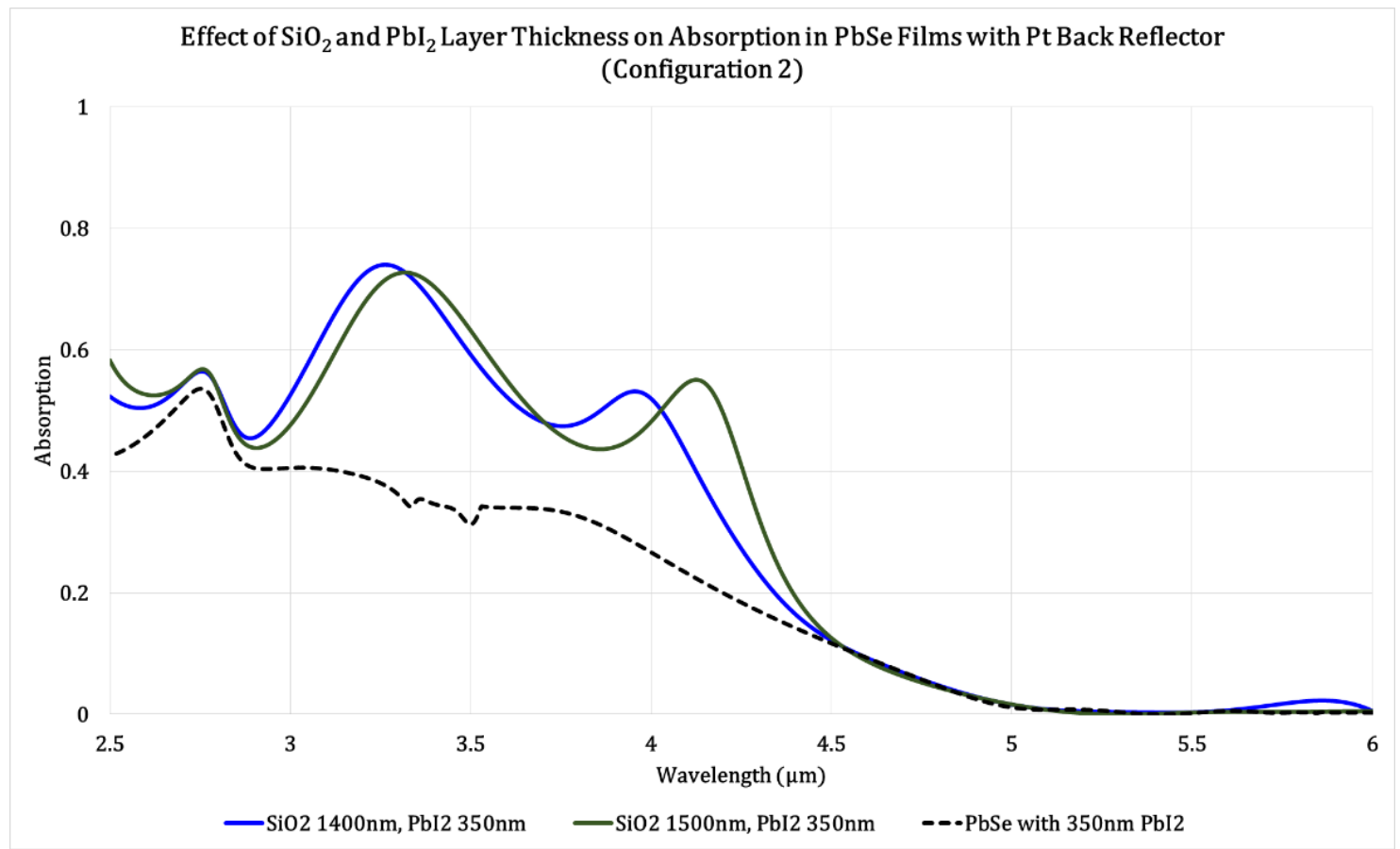

Figure 63. Effect of $\mathrm{SiO}_{2}$ and $\mathrm{PbI}_{2}$ layer thickness on absorption in PbSe Films with Pt back reflector in configuration 2.

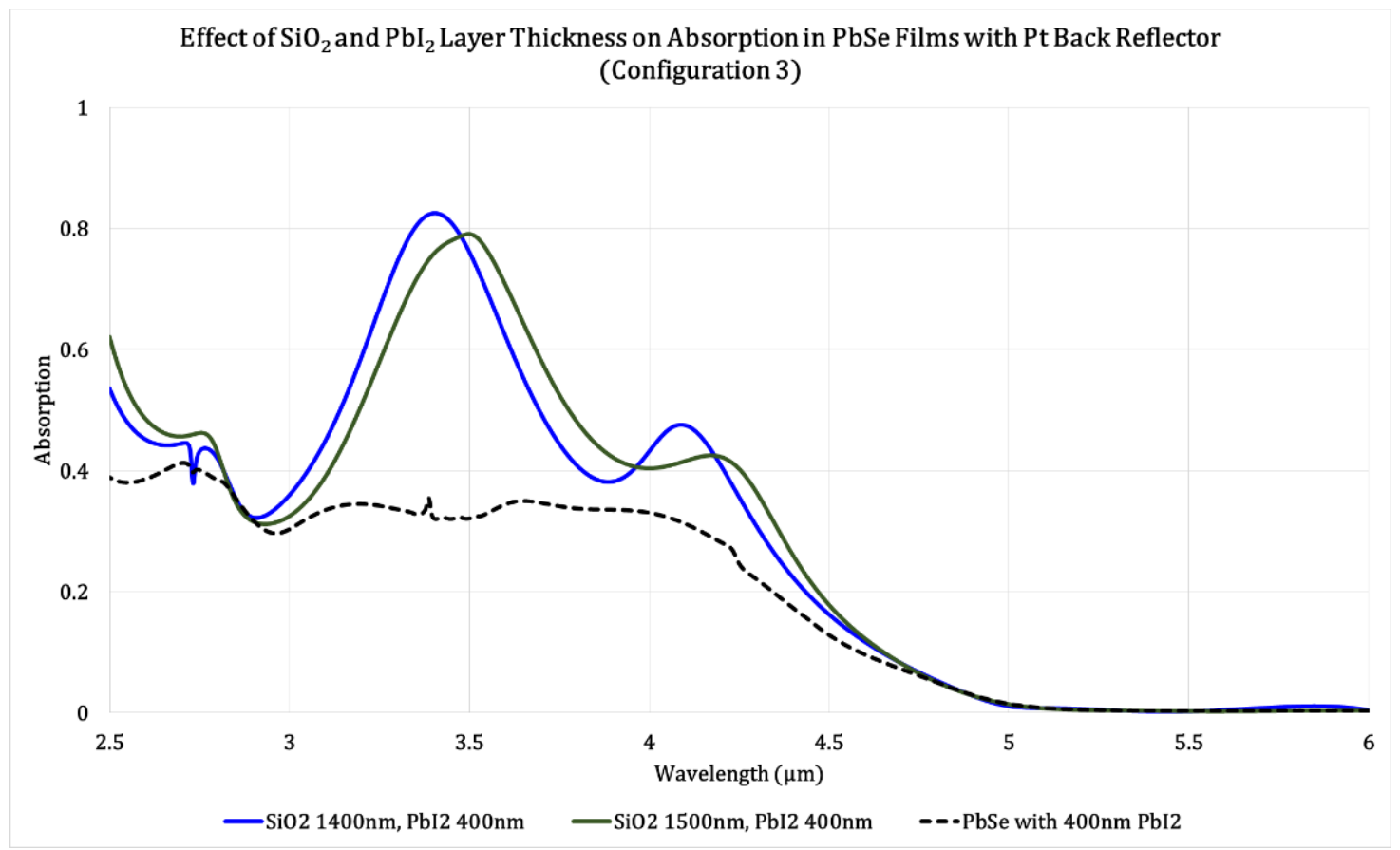

Figure 64. Effect of $\mathrm{SiO}_{2}$ and $\mathrm{PbI}_{2}$ layer thickness on absorption in PbSe Films with Pt back reflector in configuration 3. 

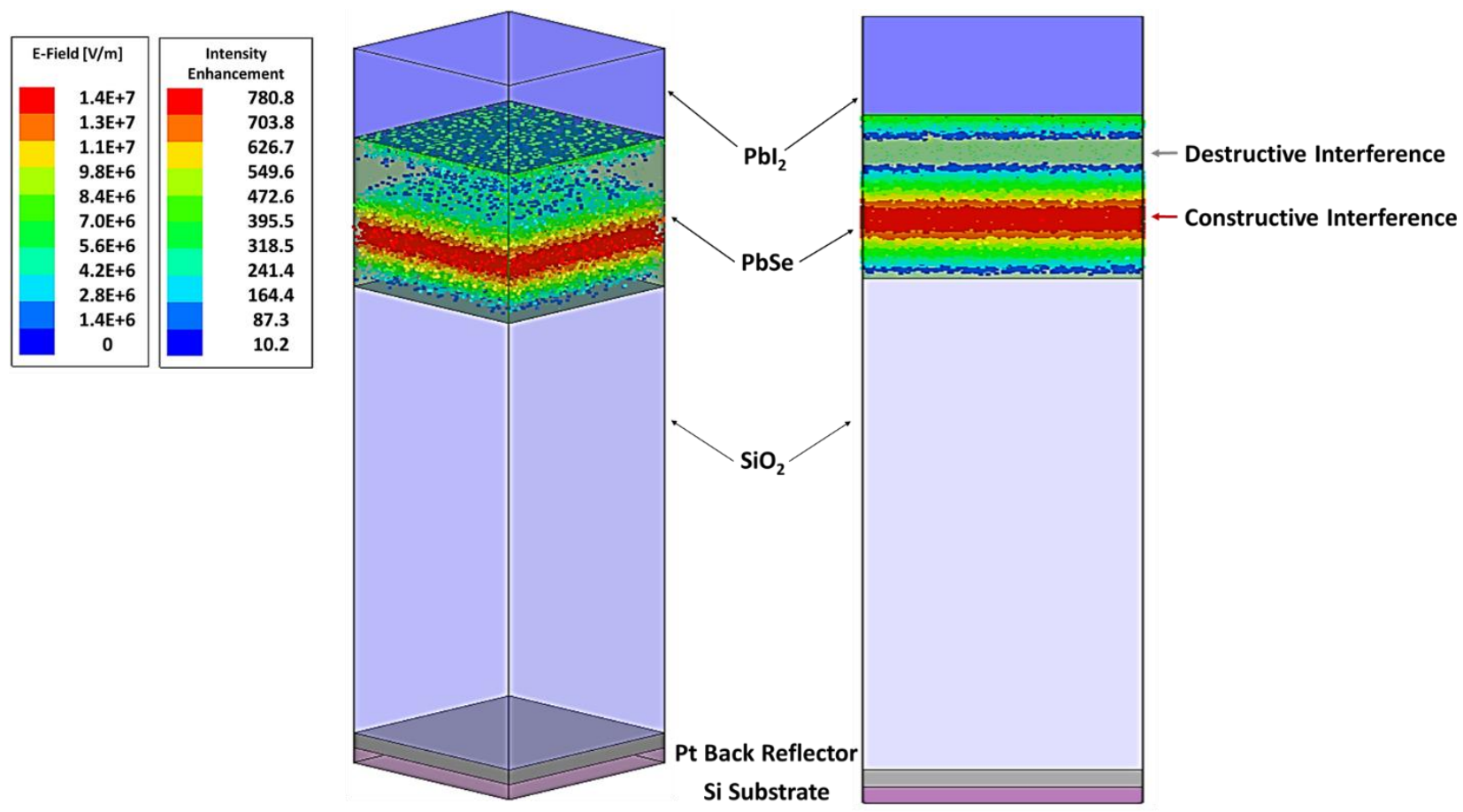

Figure 65. Electric field and field intensity enhancement due to interference at $4.08 \mu \mathrm{m}$ in PbSe films with Pt back reflector, $1500 \mathrm{~nm} \mathrm{SiO}$, and $300 \mathrm{~nm} \mathrm{PbI}_{2}$ thickness

Consider configuration 1 in figure 62 above. The peak near the absorption edge of $\mathrm{PbSe}$ $(\sim 4.3 \mu \mathrm{m})$ is $68 \%$ at $4.08 \mu \mathrm{m}$. This is nearly a factor of three enhancement over the corresponding $\mathrm{PbSe}$ baseline film at the same wavelength. To further investigate this test case, I simulated the electric field intensity in the PbSe layer of the modeled film. Flouquet ports were used for a discrete frequency sweep in which the fields were saved at every $1000 \mathrm{GHz}$ between $50000 \mathrm{GHz}$ and $120000 \mathrm{GHz}$. Recall that the $\mathrm{TE}_{00}$ and $\mathrm{TM}_{00}$ mode excitations of the Flouquet port are effectively plane waves, and in this simulation were set to an input power of $1 \mathrm{~W}$. With the unit cell area being $500 \mathrm{~nm} \times 500 \mathrm{~nm}$, the incident intensity was $4 \times 10^{12} \mathrm{~W} / \mathrm{m}^{2}$. Figure 65 shows the magnitude of the electric field within the PbSe layer, as well as the field intensity enhancement. From top to bottom, the magnitude of the field within the layer starts at $7 \mathrm{MV} / \mathrm{m}$, and then the field vanishes in the top half of the layer. This disappearance is indicative of destructive interference. 
Indeed, shortly after the field returns and results in a highly intense region with a field of $14 \mathrm{MV} / \mathrm{m}$ near the bottom half of the film, which is due to constructive interference. The predicted electric field intensity enhancement in this region is as large as 780 times that of the incident wave, showing the benefits of this simple configuration. A similar intensity increase would be seen at the $3.11 \mu \mathrm{m}$ peak; and even in the band between 3.5 $\mu \mathrm{m}$ and $4 \mu \mathrm{m}$ where the absorption decreases, a small increase over the baseline films is expected.

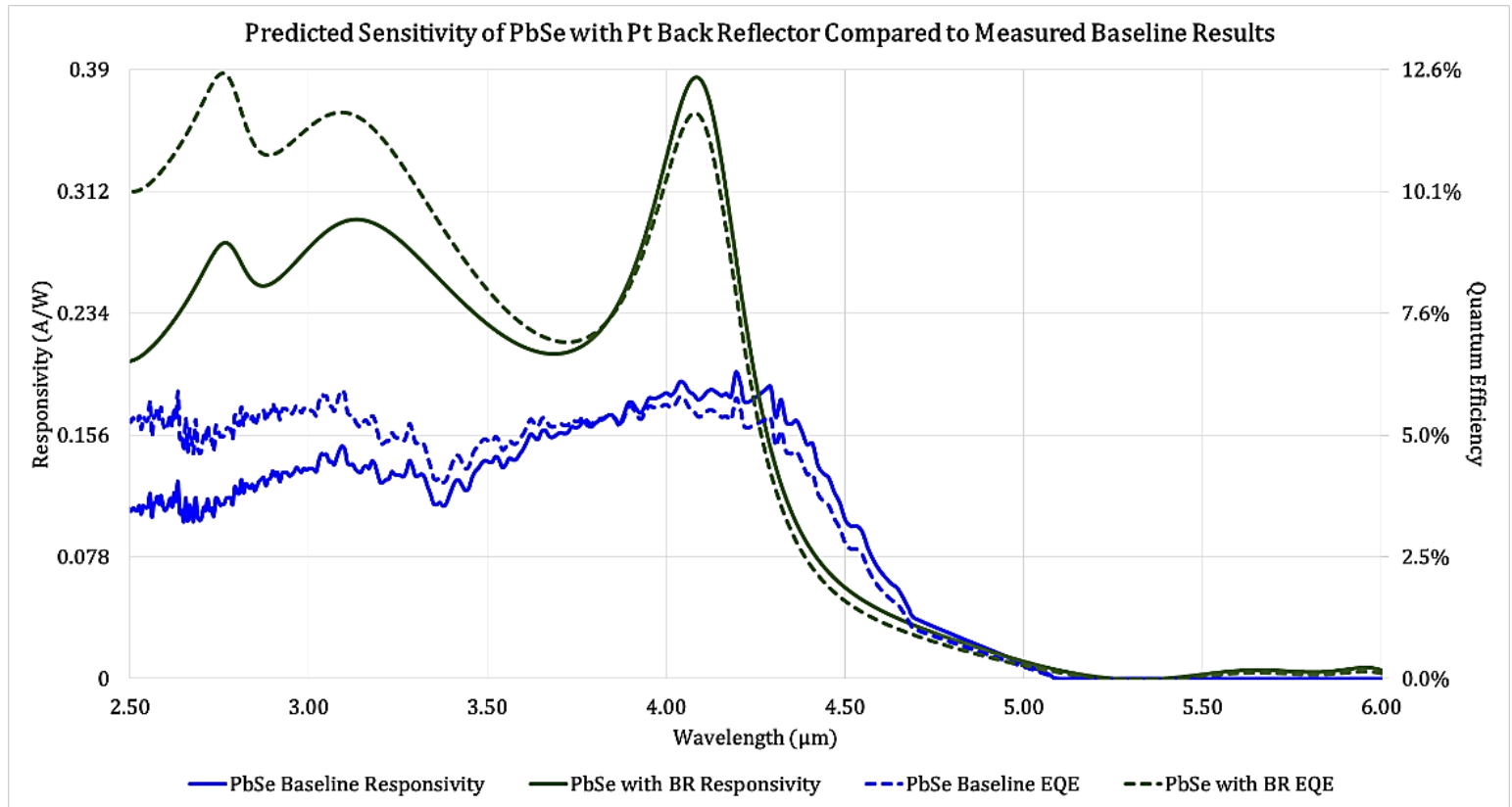

Figure 66. Predicted sensitivity of PbSe detector with Pt back reflector compared to baseline result.

So far, the primary figure of merit discussed for these films has been absorption. However for detectors, responsivity and external quantum efficiency (EQE) were used to gauge the sensitivity; after all, the goal of this investigation was to demonstrate $\mathrm{PbSe}$ device sensitivity enhancement such that the room temperature performance is comparable to that of the baseline operated at lower temperatures. Recall from figure 22 that the peak room temperature responsivity and $\mathrm{EQE}$ at $4.3 \mu \mathrm{m}$ for the baseline $\mathrm{PbSe}$ 
detectors was shown to be $0.2 \mathrm{~A} / \mathrm{W}$ and $6 \%$, respectively. Coupled with figure 20 , which shows the PbSe absorption at the absorption edge $(\sim 4.3 \mu \mathrm{m})$ is $35 \%$, the internal quantum efficiency (IQE), which is the quotient of the EQE and absorption, for these detectors at room temperature is calculated to be $17.14 \%$. I used this IQE to calculate the responsivity from equation 2.15 in Chapter 2 based on the predicted absorption.

The predicted sensitivity for a photoconductor derived from a back reflector film with a $300 \mathrm{~nm} \mathrm{PbI} I_{2}$ layer is shown in figure 66 . The dip in responsivity at $\sim 3.4 \mu \mathrm{m}$ in the baseline detector is predicted to red-shift to $\sim 3.5 \mu \mathrm{m}$ in the back reflector enhanced films. Further, there are three distinguishable peaks at approximately $2.8,3.3$, and $4.1 \mu \mathrm{m}$. It would seem that due to resonance the large peak at $4.08 \mu \mathrm{m}$ blue shifts the response spectrum compared to baseline $\mathrm{PbSe}$. This is evidence of interference, because the wavelengths that match the longitudinal modes of the film will experience the largest absorption enhancement in a plane-parallel cavity. However, as will be shown in Chapter 6, this peak was observed to be much wider due to the roughness of the deposited films. Roughness leads to peak broadening, and, as discussed in Chapter 2, the measured films are very rough compared to the smooth interfaces that were modeled. Of note is the increase in sensitivity over the whole band. At the $4.08 \mu \mathrm{m}$ peak, the sensitivity is increased by a factor of 2, and over the whole band the responsivity and quantum efficiency are well above that of the baseline detectors. These results predict the responsivity and quantum efficiency of PbSe detectors with embedded back reflectors are indeed comparable to those of the baseline films at lower temperatures. These results were put to the test, and in the next chapter it will be demonstrated that the back reflector enhanced $\mathrm{PbSe}$ detectors offer a major improvement over the current state-of-the-art. 


\section{Chapter 5 References}

[1] K. K. Choi, et al. "Electromagnetic modeling and resonant detectors and arrays," Infrared Physics \& Technology, Vol. 70, 2015.

[2] K. K. Choi, et al. "Resonant structures for infrared detection," Applied Optics, Vol. 56, No. 3, 2017.

[3] K. K. Choi, et al. "Resonator-quantum well infrared photodetectors," Appl. Phys. Lett. 103, 2013.

[4] Getting Started with HFSS: Optimizing a Waveguide T Junction. ANSYS Release 18.2, ANSYS, Inc., 2017.

[5] ANSYS Metamaterial Summit: Frequency Selective Surfaces. ANSYS 14.0 Release, ANSYS, Inc., 2012.

[6] Workshop 3-6: RCS of a Cube. ANSYS 2015.0 Release, ANSYS, Inc., 2015.

[7] N. M. Buford, "Plasmonic Nanostructurres for the Absorption Enhancement of Silicon Solar Cells," Masters Thesis, University of Arkansas, 2013.

[8] Material Properties. ANSYS, Inc., 2014.

[9] HFSS Fields Calculator Cookbook. ANSYS Release 17.2, ANSYS, Inc., 2016.

[10] Modeling Layered Structures with Shell Elements. ANSYS, Inc., 2016.

[11] K. F. Warnick, Numerical Methods for Engineers: An Introduction Using MATLAB and Computational Electromagnetics Examples, SciTech Publishing, Edison, NJ, 2011.

[12] M. N. O. Sadiku, "A Simple Introduction to Finite Element Analysis of Electromagnetic Problems," IEEE Transactions on Education, Vol. 32, No. 2, 1989. 
[13] M. Jones, “Ansys High Frequency Structure Simulator (HFSS) Tutorial,” Pacific Northwest National Laboratory, 2017. [Online]. Available:

https://indico.fnal.gov/event/13068/contribution/10/material/slides/1.pdf. [Accessed: Nov. 3, 2018].

[14] ANSYS, Inc., An Introduction to HFSS, Release 18.2, Canonsburg, PA: ANSYS, Inc., 2017.

[15] J. Hoffmann, et al. "Comparison of electromagnetic field solvers for the 3D analysis of plasmonic nano antennas," Proc. SPIE. Vol. 7390, 2009.

[16] C. A. Balanis. Advanced Engineering Electromagnetics, $1^{\text {st }}$ Ed., Hoboken, NJ: John Wiley and Sons, Inc. 1989.

[17] ANSYS, Inc., "Appenix 5-2: HFSS 3D Boundary Conditions," Technical Note, Release 2014.0, Canonsburg, PA: ANSYS, Inc., 2014.

[18] ANSYS, Inc., "Introduction to ANSYS HFSS for Antenna Design", Presented as part of FSS Workshop, ANSYS 14.0 Release, ANSYS, Inc., 2012.

[19] ANSYS, Inc., "Getting Started with HFSS: Floquet Ports," Technical Note, Release 18.2, Canonsburg, PA: ANSYS, Inc., 2017.

[20] J. O. Sophocles, Electromagnetic Waves and Antennas, Rutgers University, 2016.

[21] Agilent Technologies, Inc., "Topic 5: S-parameter Simulation and Optimization." ADS 2009 (version 1.0). Agilent Technologies, Inc., 2009.

[22] ANSYS, Inc., "HFSS with HPC for Large Finite Antenna Array Design," Canonsburg, PA: ANSYS, Inc., 2011. 
[23] D. Sarid and W. Challner, Modern Introduction to Surface Plasmons: Theory, Mathematical Modeling, and Application, New York, NY: Cambridge University Press, 2010.

[24] F. M. Limonov, et al. "Fano resonances in photonics," Nature Photonics, Vol. 11, 2017.

[25] Y. Zhang, et al. "Fano resonances in disc-ring plasmonic nanostructure: strong interaction between bright dipolar and dark multipolar mode," Optics Letters, Vol. 37, No. 23, 2012.

[26] B. Luk'yanchuk, et al. "The Fano resonance in plasmonic nanostructures and metamaterials," Nature Materials, Vol. 9, 2010.

[27] X. Tang, et al. "Plasmon resonance enhanced colloidal HgSe quantum dot filterless narrowband photodetectors for mid-wave infrared," J. Mater. Chem. C, $5,2017$.

[28] E. Hecht, Optics, 4th Edition. Addison-Wesley, New York, 2002.

[29] R. E. Fischer, et al. Optical System Design, 2nd Edition, SPIE Press, McGraw Hill, 2008.

[30] I. Chambouleyron and J. M. Marinez, Handbook of Thin Films Materials; ch 12, edited by Nalwa, H.S., Academic Press, 2001. 


\section{Chapter 6: PbSe Film and Device Measurements}

The results presented thus far have assumed smooth interfaces, perfect fabrication and ideal conditions. To determine the achievable enhancement under laboratory conditions, structures were fabricated in PbSe films and test detector devices. The detector fabrication procedures are described in Appendix A. In this chapter, the experimental results of the investigated $\mathrm{PbSe}$ devices with embedded resonant structures are discussed.

\subsection{Confirmation of Plasmonic Resonance of Metal Discs in PbSe}

Before devices with embedded plasmonic structures were fabricated using photolithography, I performed preliminary studies to verify the feasibility of metallic discs in PbSe. With help from my collaborators, Au and Pt discs were fabricated on the $\mathrm{SiO}_{2}$ layer using electron beam lithography (EBL), which is similar to photolithography with the exception that the exposure is with a concentrated electron beam instead of UV photons [1-2]. We patterned $500 \mathrm{~nm}$ and $1000 \mathrm{~nm}$ diameter discs into $1 \mathrm{~mm} \times 1 \mathrm{~mm}$ arrays with a pitch, defined as the center-to-center disc spacing, twice that of the diameter. After patterning and developing the e-beam resist, RF sputtering was performed to deposit a chromium $(\mathrm{Cr})$ adhesion layer and either Pt or Au onto the substrate. In accordance with table 9, the deposited thickness of the Pt and Au discs was selected such that the aspect ratio would be 12.5 and 30, respectively. Liftoff and cleaning was then performed to remove excess metal and contaminates from the surface. Figure 67 shows scanning electron microscope images of the arrays, and table 11 shows the measured disc diameters and pitches. 
$500 \mathrm{~nm}$ diameter discs with $1000 \mathrm{~nm}$ pitch

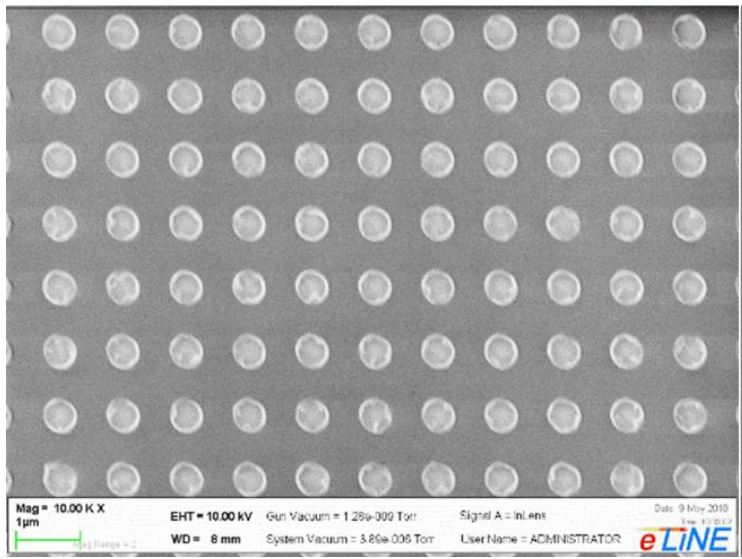

$1000 \mathrm{~nm}$ diameter discs with $2000 \mathrm{~nm}$ pitch

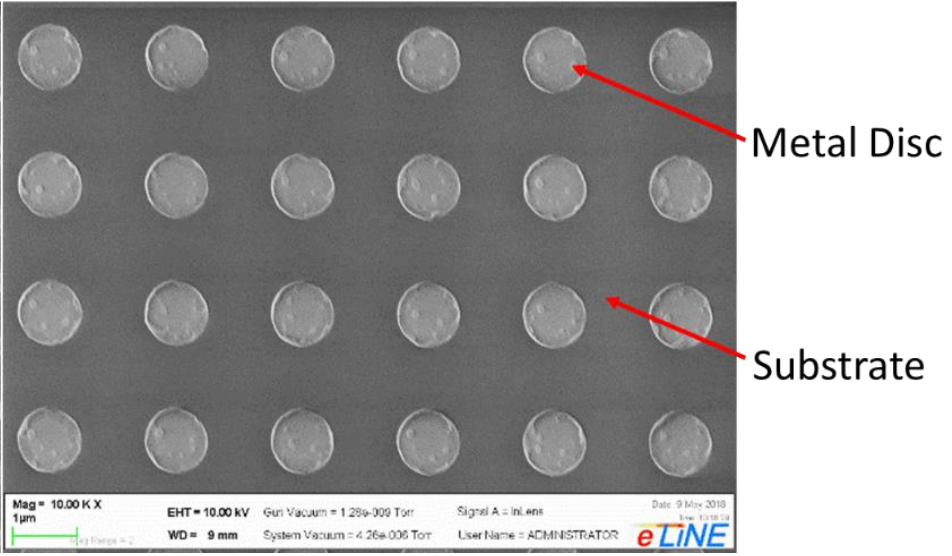

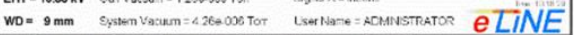

Figure 67. SEM images of $500 \mathrm{~nm}$ (left) and $1000 \mathrm{~nm}$ (right) disc arrays patterned by EBL.

Table 11. Measured disc diameter and pitch for $500 \mathrm{~nm}$ and $1000 \mathrm{~nm}$ Au and Pt discs patterned by EBL.

\begin{tabular}{|c|c|c|c|c|c|}
\hline Metal & $\begin{array}{c}\text { Designed } \\
\text { Dia./Pitch } \\
(\mathbf{n m})\end{array}$ & $\begin{array}{c}\text { Measured } \\
\text { x-Dia.(nm) }\end{array}$ & $\begin{array}{c}\text { Measured } \\
\text { y-Dia. (nm) }\end{array}$ & $\begin{array}{c}\text { Measured } \\
\text { x-Pitch (nm) }\end{array}$ & $\begin{array}{c}\text { Measured } \\
\text { x-Pitch (nm) }\end{array}$ \\
\hline $\mathrm{Pt}$ & $500 / 1000$ & 602.9 & 502.4 & 1016.0 & 846.6 \\
\hline $\mathrm{Pt}$ & $1000 / 2000$ & 982.5 & 960.2 & 1998.5 & 1953.1 \\
\hline $\mathrm{Au}$ & $500 / 1000$ & 524.8 & 558.3 & 937.8 & 848.6 \\
\hline $\mathrm{Au}$ & $1000 / 2000$ & 993.8 & 1027.0 & 1965.0 & 1942.8 \\
\hline
\end{tabular}

Before depositing PbSe onto the disc arrays, I used a Thermo Fisher Scientific iS5 tabletop FTIR spectrometer to determine the optical density spectra of the disc arrays. It was important to measure the baseline spectrum from the disc arrays alone for comparison with disc arrays embedded in $\mathrm{PbSe}$. In this case, the integrating sphere method described in Chapter 2 was not used due to the small area of the disc arrays. Instead, the discs were positioned over a $2 \mathrm{~mm}$ aperture to ensure the focused black body source would be incident only on the array. The background transmission signal was collected at normal incidence through the aperture alone, and the signal transmission was collected through the disc arrays and aperture. The optical density was calculated using equation 2.13 found in Chapter 2. 


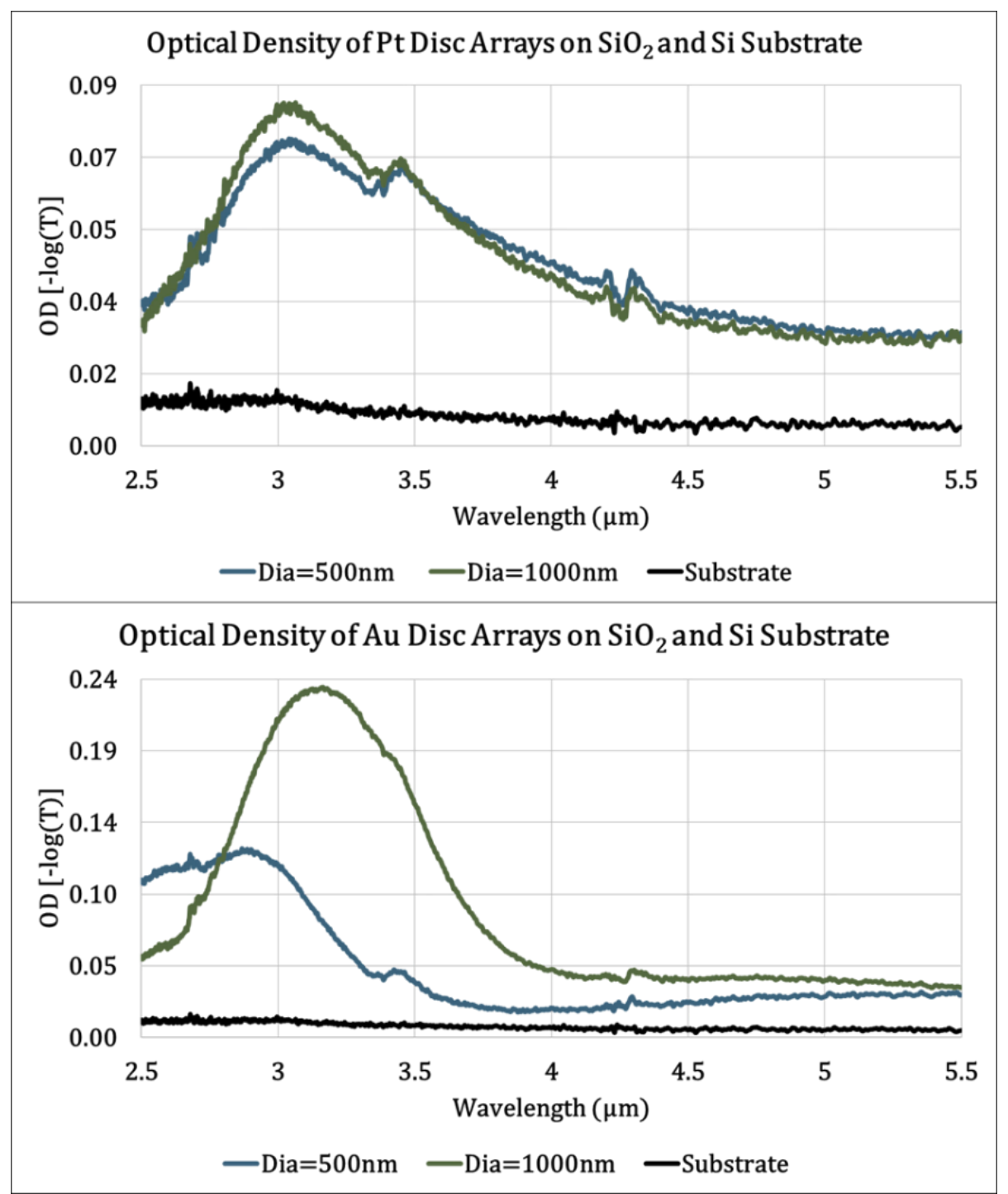

Figure 68. Optical density spectra of Pt (top) and $\mathrm{Au}$ (bottom) disc arrays on $\mathrm{SiO}_{2}$ layer and Si substrate.

Figure 68 shows the measured OD spectra of the Pt and Au disc arrays.

Considering the $1000 \mathrm{~nm}$ discs the width of the Pt peak was observed to be approximately $1.25 \mu \mathrm{m}$. In contrast the width of the Au peak is smaller at $0.71 \mu \mathrm{m}$. The difference in the peak width is expected due to the longer surface plasmon lifetime shown in figure 33. A more interesting phenomenon is noticed when comparing the difference in how the spectra change with size between the Pt and Au disc arrays. For Pt the size seems to only have the effect of increasing the amplitude, which follows the general rule that 
aspect ratio is the dominant parameter for SPR in discs and is consistent with figure 53. However, in Au the resonance shifts to longer wavelengths as the disc size increases. This behavior is in contrast with figure 52. The explanation again comes back to the conductivity of the two metals. As seen in figure 57, Au has a higher electrical conductivity, and therefore is less lossy than Pt. Less loss equates to higher reflectivity, and makes $\mathrm{Au}$ act like a perfect electric conductor (PEC) in the MWIR regime.

Therefore, aspect ratio, more specifically the thickness of the disc, is not as important a parameter as the diameter for the resonance in Au discs. This explanation seems to be consistent with the modeled results of figure 56.

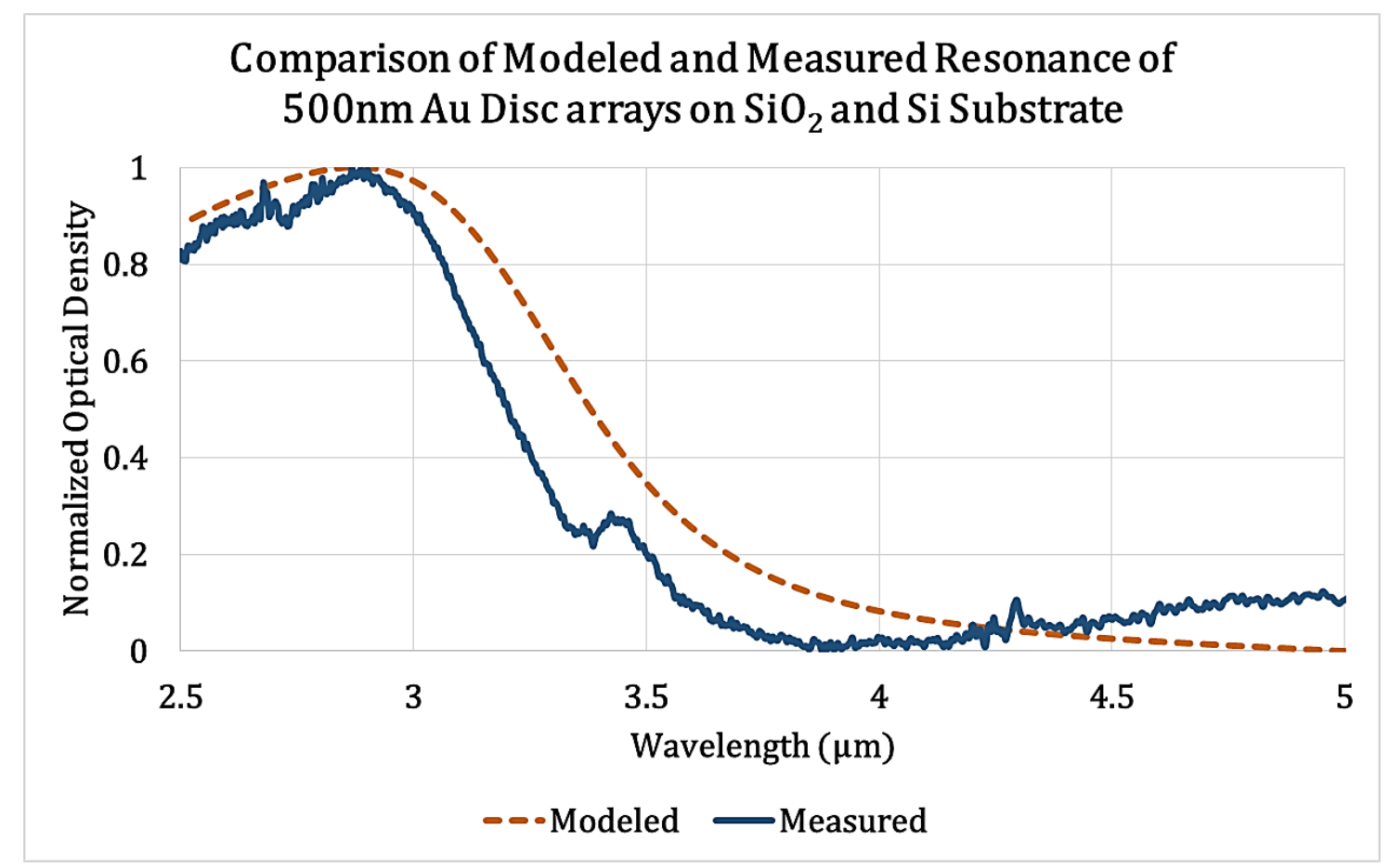

Figure 69. Comparison of modeled and measured results for $500 \mathrm{~nm} \mathrm{Au} \mathrm{disc} \mathrm{array} \mathrm{on} \mathrm{SiO}_{2}$ layer and $\mathrm{Si}$ substrate at room temperature.

Also of note in figure 68 is the differences in the amplitude of the OD. A comparison of the $500 \mathrm{~nm}$ diameter disc arrays will show that the amplitude of $\mathrm{Au}$ is 
slightly larger than that of Pt. This again is to be expected due to the larger electrical conductivity of $\mathrm{Au}$. Yet, the Au has more of an asymmetric resonance. To confirm that this asymmetry was not an anomaly in the measurement, I modeled the structures in ANSYS HFSS. Figure 69 shows that the modeled results agree well with the measurement, and seem to support the assertion made in Chapter 5 that Au will yield a more asymmetric resonance than Pt.

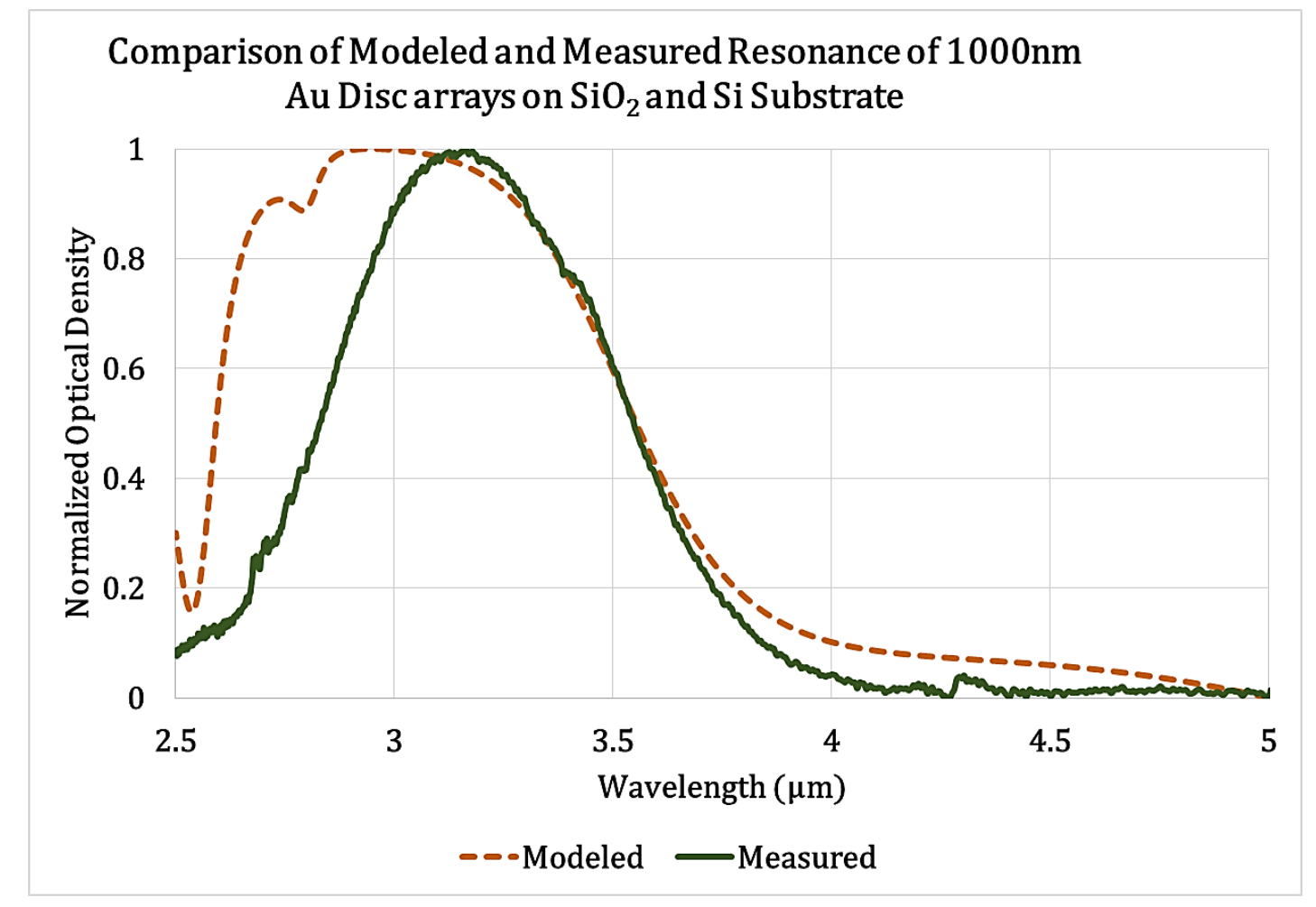

Figure 70. Comparison of modeled and measured results for $1000 \mathrm{~nm}$ Au disc array on $\mathrm{SiO}_{2}$ layer and $\mathrm{Si}$ substrate at room temperature.

In contrast, the larger discs for $\mathrm{Au}$, shown in figure 70 , have a more symmetric line shape. Again, I modeled these discs to confirm the legitimacy of my measurements. The modeled results show a broader resonance than that of the measured, however the two spectra are still in reasonable agreement. Both show a more symmetric resonance, yet their peaks are shifted with respect to one another by $170 \mathrm{~nm}$, suggesting the 
deposited metal discs may have a slightly different diameter than designed. Examining the measured disc diameters in table 11 seems to confirm my hypothesis, because the discs were not perfectly circular.

Our PbSe films were deposited, oxidized, and sensitized on the $500 \mathrm{~nm}$ Au disc array, and the OD was measured in the same method described above. The $500 \mathrm{~nm} \mathrm{Au}$ disc array was chosen for these measurements instead of the $1000 \mathrm{~nm}$ disc array to avoid the interference peaks observed in the modeled results in figure 61. Due to the roughness of the films and the substrate, interference effects are difficult to distinguish during a simple FTIR OD measurement. Therefore, larger discs are reserved for $\mathrm{PbSe}$ devices described later.

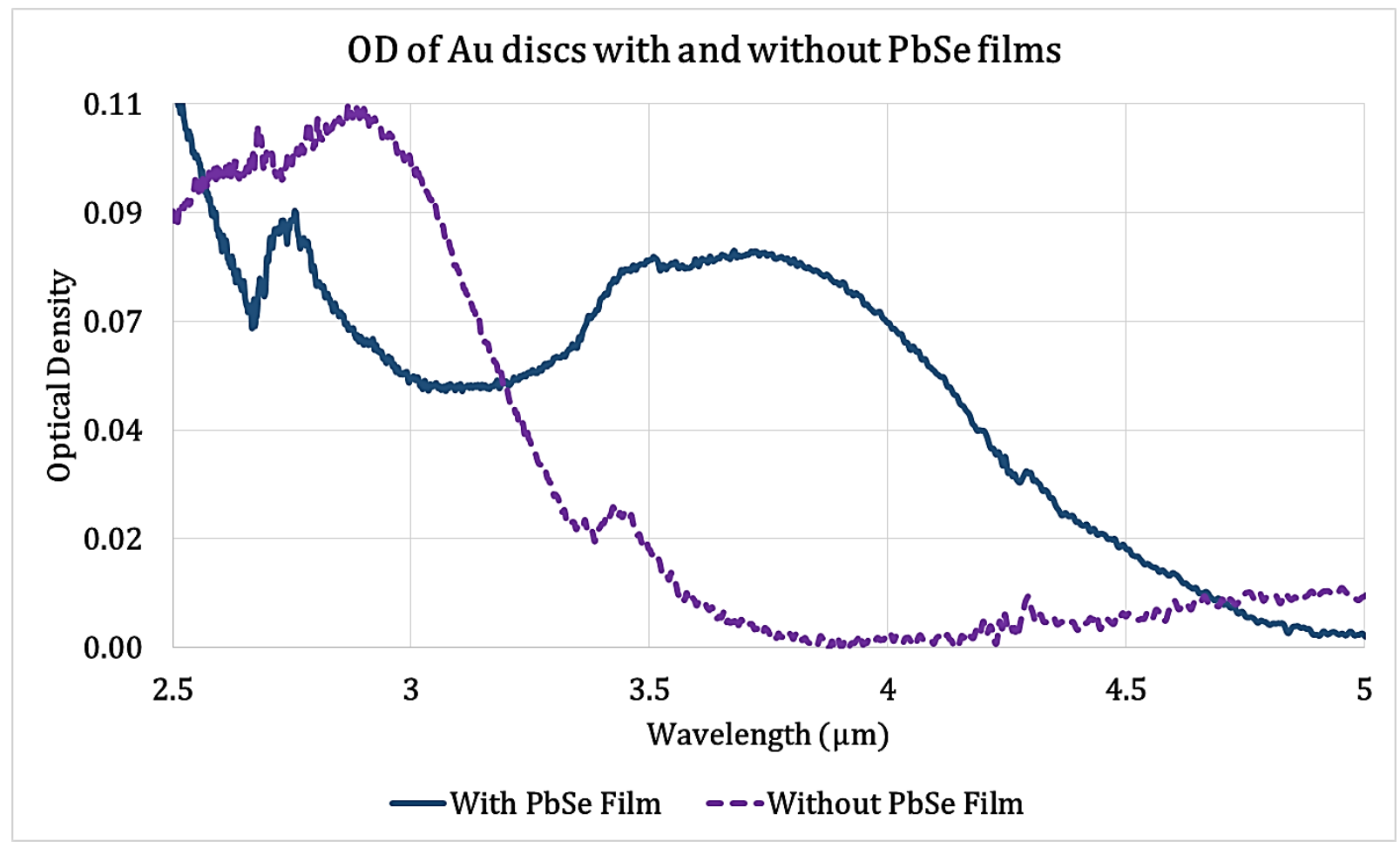

Figure 71. Room temperature comparison of resonance for $500 \mathrm{~nm}$ diameter Au disc arrays within and without PbSe films with layer thicknesses of approximately $400 \mathrm{~nm}, 500 \mathrm{~nm}$, and $1200 \mathrm{~nm}$ for the PbI $\mathrm{I}_{2}$ $\mathrm{PbSe}$, and $\mathrm{SiO}_{2}$, respectively. 
Figure 71 shows the spectra obtained from $\mathrm{PbSe}$ films with embedded discs. Inside the $\mathrm{PbSe}$ films, the resonance shifts by $860 \mathrm{~nm}$ from the discs without $\mathrm{PbSe}$. Further, the amplitude of the optical density decreases due to the decrease in reflectance between the $\mathrm{PbSe}-\mathrm{Au}$ interface compared to the air-Au interface without $\mathrm{PbSe}$ films. This shift, as well as the resonance peak wavelength, is indicative of surface plasmon resonance. Moreover, this shift was predicted when considering the discs modeled without $\mathrm{PbSe}$ shown in figure 69, and the disc arrays modeled with $\mathrm{PbSe}$ shown figure 56. This shift confirms the surface plasmon resonance effect present in the investigated $\mathrm{PbSe}$ films with embedded metallic discs.

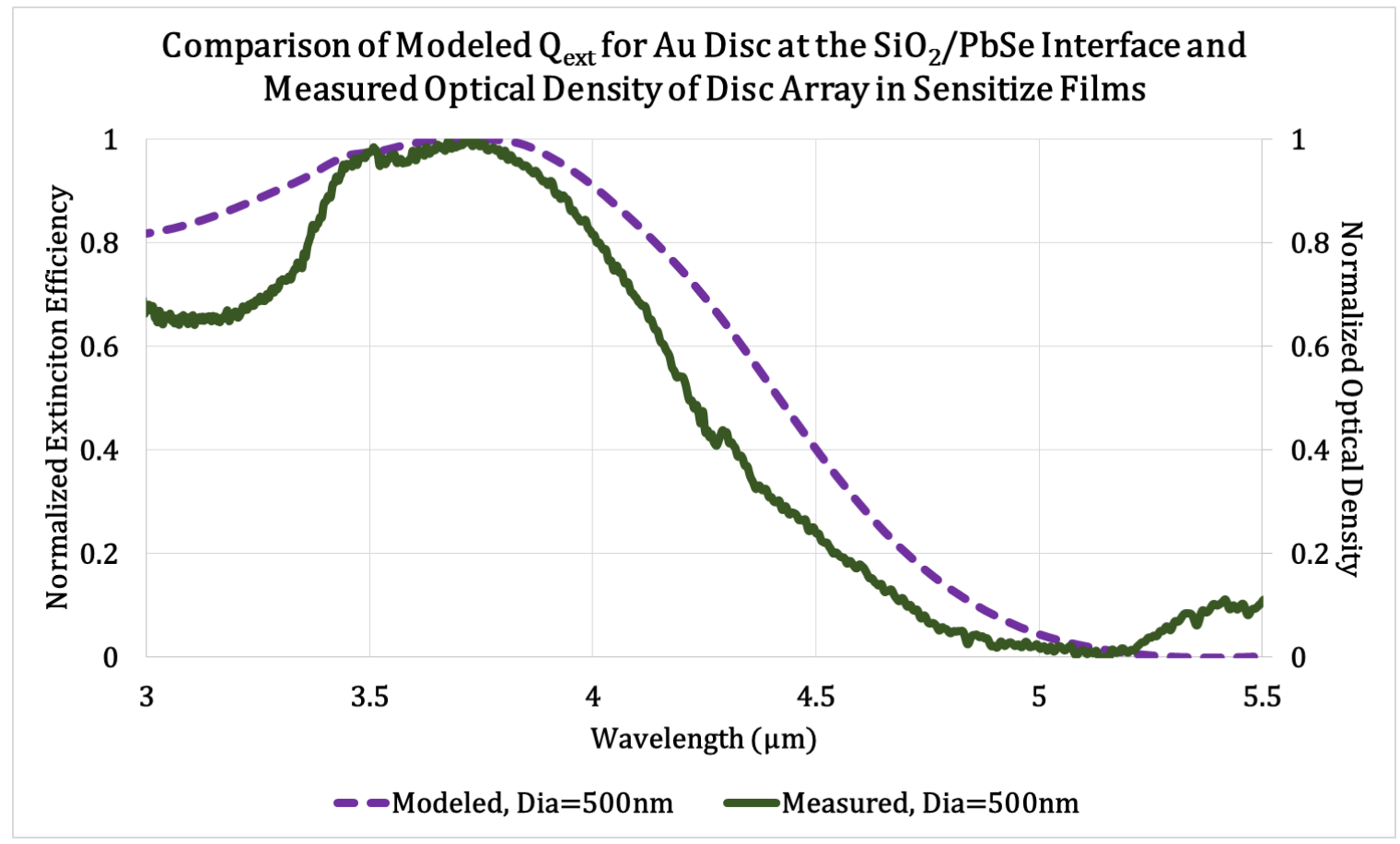

Figure 72. Comparison of modeled and measured spectra of $500 \mathrm{~nm}$ Au disc arrays in PbSe films.

For completeness, figure 72 shows the comparison between modeled extinction efficiency in figure 56 and the measured OD. The peak of the measured and modeled spectra match, suggesting that a pitch twice that of the diameter leads to a negligible 
amount of coupling between the fields of adjacent discs. The measured discs show a narrower resonance than that of the modeled spectra, similar to the results presented in figure 69 and figure 70. This increased broadening in the modeled results is most likely due to the difference in the dispersion relations of the real film and fabricated gold discs. Nevertheless, the peak resonance was accurately predicted, so the next step was to fabricate and measure $\mathrm{PbSe}$ photoconductive devices with embedded discs.

\subsection{PbSe Detectors with Embedded Pt Disc Arrays}
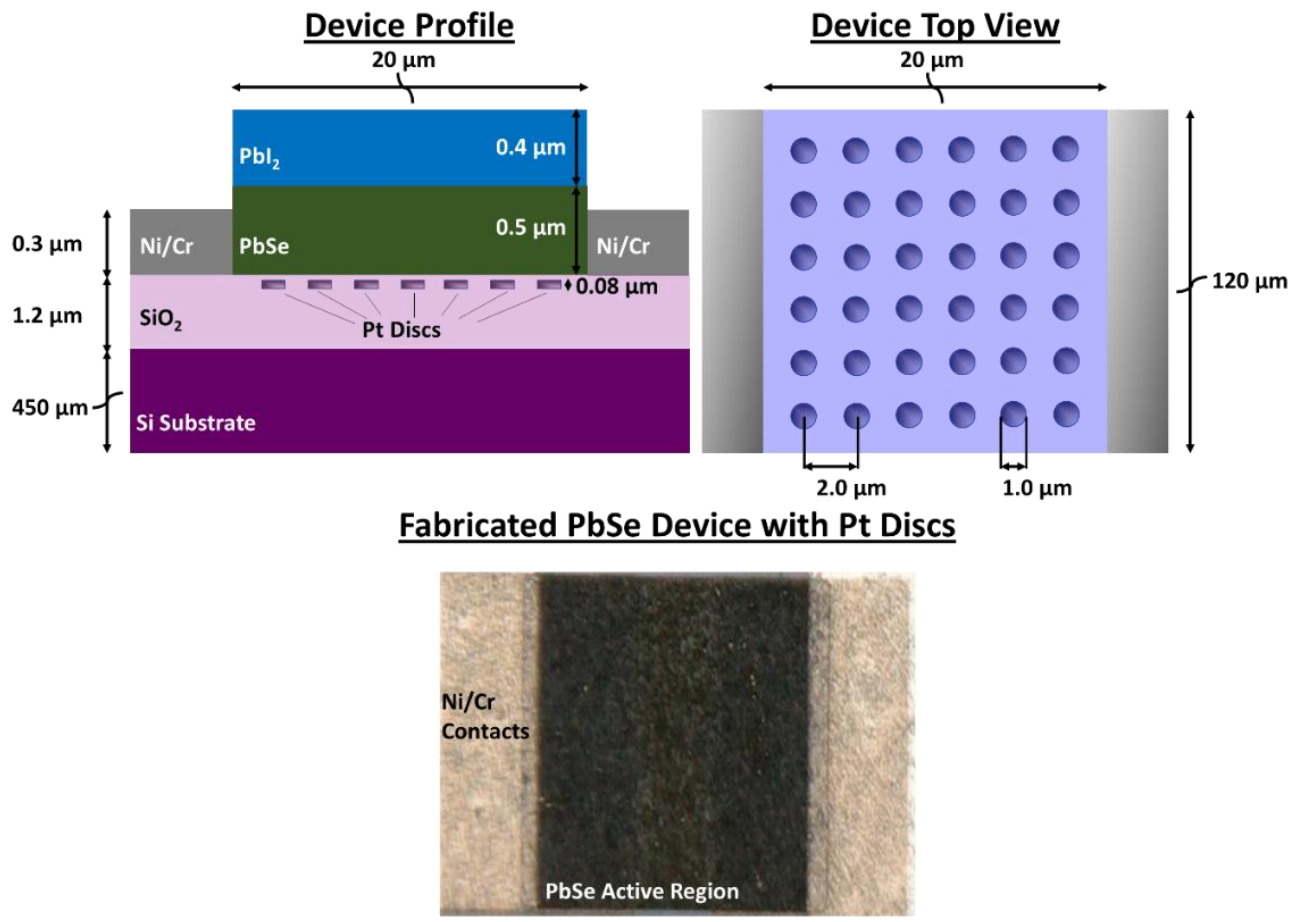

Figure 73. PbSe photoconductive devices with embedded Pt disc array; (top-left) depiction of the designed device cross-section, (top-right) depiction of the top view of device showing the Pt disc arrays, and (bottom) top view of fabricated PbSe device.

Test detector devices with embedded $\mathrm{Pt}$ discs at the $\mathrm{SiO}_{2}-\mathrm{PbSe}$ interface were fabricated using photolithography. Gold, though it offers a larger enhancement and is acceptable for FTIR measurements, was found to have a tendency to thermally diffuse into the PbSe during the oxidation and sensitization processes. This has been found in 
previous experiments to lead to leakage current and overall degraded performance in photoconductive PbSe devices. Platinum did not show any signs of diffusion, and so was used for this investigation. Platinum has a higher loss than $\mathrm{Au}$, so the performance would prove to be different than that predicted in figure 61 . The resolution of the photomask and photolithography system was limited to $\sim \mu \mathrm{m}$ features, so discs of approximately $1 \mu \mathrm{m}$ and aspect ratio between 12.5 and 15 were fabricated. Here the variation in aspect ratio was due to variances in fabricated thickness. Test detectors, depicted in figure 73, were $20 \mu \mathrm{m}$ (width) $\mathrm{x} 110 \mu \mathrm{m}$ (length) in dimension for the disc detectors. To reduce the possibility of quenching, a $\mathrm{SiO}_{2}$ insulation layer was deposited on top of the discs. For these devices the insulation layer was designed to be $30 \mathrm{~nm}$, however through fabrication errors the insulation layer turned out to be $180 \mathrm{~nm}$. This fabrication error will be discussed in more detail for it is the primary reason that the measurement results did not agree with the modeled spectrum.

I measured the spectral response of these PbSe photoconductive devices with embedded Pt discs using a modified method as that depicted in Chapter 2. For these measurements a FEMTO model DLPCA-200 current pre-amplifier was connected in series with the detector, and was used to bias the detector up to a maximum value of $6 \mathrm{~V}$ for a field strength, defined as the quotient of the applied voltage and width of the active region between metal contacts, of $3 \mathrm{kV} / \mathrm{cm}$. This pre-amplifier was operated in AC mode, and grounded to the test fixture chassis to reduce noise. Signal current was amplified by a factor of $10^{6}$, and input into a Stanford Research Systems (SRS) model SR560 low-noise amplifier. The SRS low-noise amplifier converts the signal to voltage, and noise is further reduced by a $300 \mathrm{kHz}-1 \mathrm{MHz}$ built in bandpass filter. The signal was further amplified 
up to a factor of 100 before being output to the auxiliary detector input of a Magna-IR model 550 spectrometer. The secondary FTIR arm was used to project the black body source out of the FTIR, where it was reflected off of a parabolic mirror with a $50 \mathrm{~cm}$ focal length and focused onto the detector. Again, a Thor Labs FDPSE2X2 $2 \mathrm{~mm} \times 2 \mathrm{~mm}$ PbSe photoconductor was used to determine the power of the black body source, and using equation 2.15 from Chapter 2 the spectral responsivity was calculated.

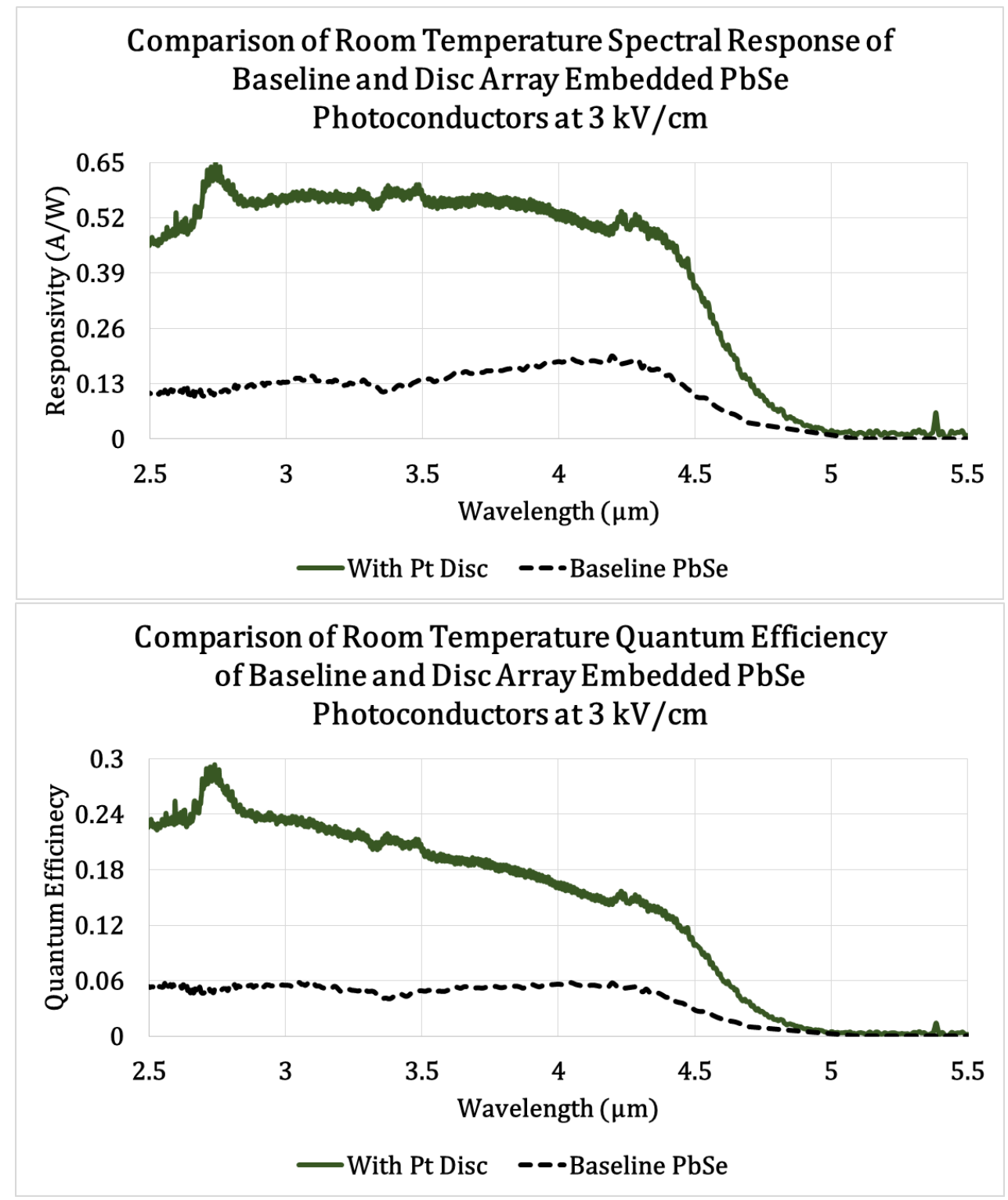

Figure 74. Comparison of room temperature (290 K) spectral response (top) and EQE (bottom) between PbSe with embedded Pt discs and baseline detectors. 

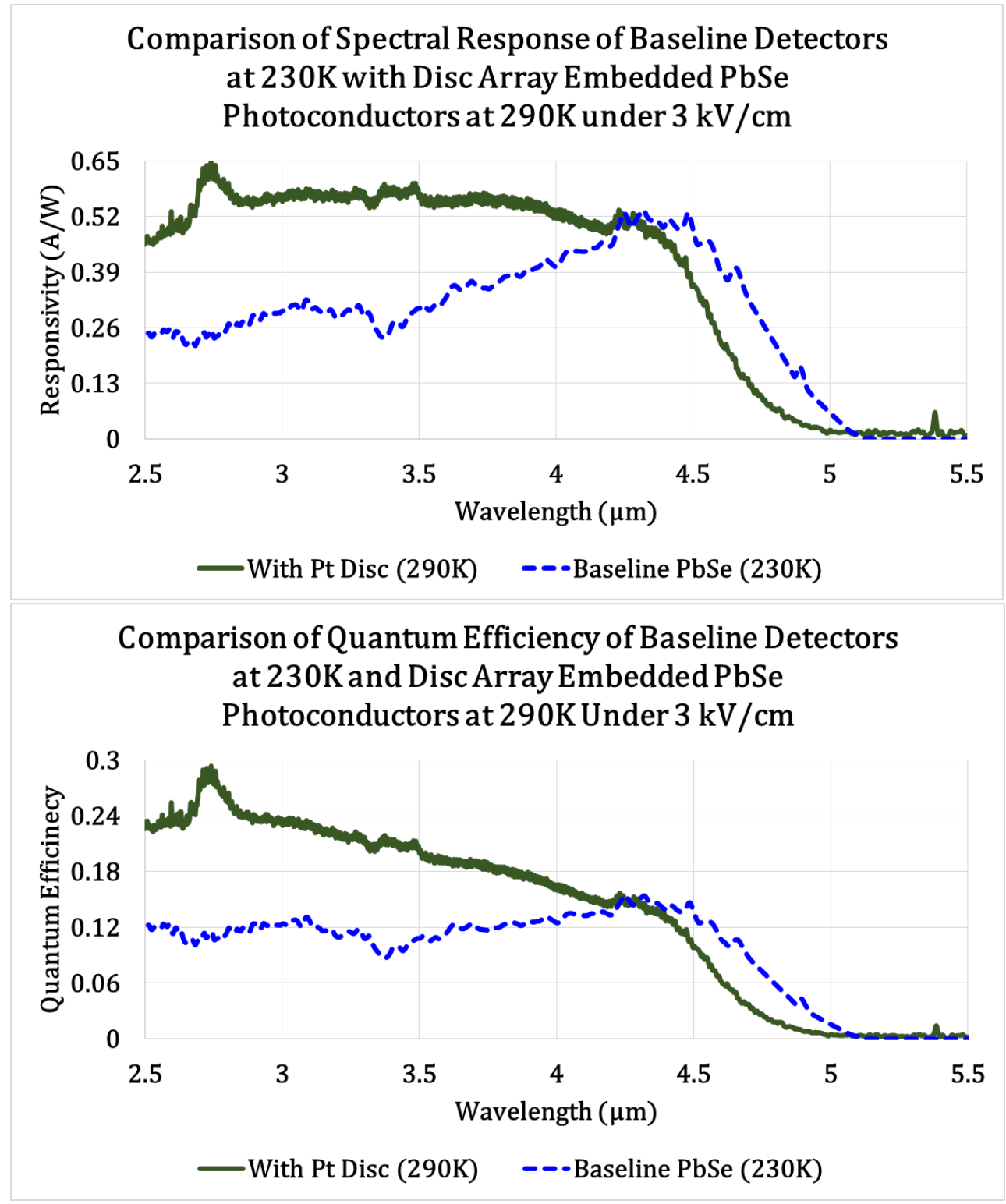

Figure 75. Comparison of room temperature $(290 \mathrm{~K})$ spectra of PbSe with embedded Pt discs with that of the $230 \mathrm{~K}$ spectra of baseline detectors.

The measured room temperature spectral response of these devices is shown in figure 74 . The maximum in-band response was found to be $570 \mathrm{~mA} / \mathrm{W}$ between $\sim 3.4 \mu \mathrm{m}$ and $3.8 \mu \mathrm{m}$ as opposed to $180 \mathrm{~mA} / \mathrm{W}$ at $4.3 \mu \mathrm{m}$ seen in the baseline detectors. This result shows a factor of three increase, as well as a blue shift in the maximum position. However, the spectrum does not show the predicted behavior of figure 61. From the 
prediction, it would be expected that there would be distinguishable interference peaks, as well as a peak close to $3.6 \mu \mathrm{m}$. This discrepancy between the measured and modeled spectrum shape can be explained by the $180 \mathrm{~nm} \mathrm{SiO}_{2}$ insulation layer. It was designed to be $30 \mathrm{~nm}$, yet through errors in fabrication it was six-times greater. Such a large layer of $\mathrm{SiO}_{2}$ deposited on top of the discs lead to the surrounding medium having a factor of two lower index of refraction than the effective index of refraction at the interface between the $\mathrm{PbSe}$ and $\mathrm{SiO}_{2}$ layers. It is the authors hypothesis, from the principles described in Chapter 3, that this would blue shift the primary resonance peaks. Furthermore, it would lead to unknown interference effects. This is a point of further investigation for future study.

Though a distinguishable peak was not observed, the decrease close to $3.4 \mu \mathrm{m}$ indicative of interference in the baseline PbSe spectrum was absent. There are two possible explanations. The first is that the difference in $\mathrm{SiO}_{2}$ thickness and the placement of the disc spoils the cavity length that leads to interference. The second possible explanation is that the peak that now resides at $\sim 3.4 \mu \mathrm{m}$ is from plasmonic resonance, which now compensates for the reduction in responsivity due to destructive interference. This second explanation would explain the factor of three increase over the baseline $\mathrm{PbSe}$ performance. It has been shown using surface plasmon resonant disc arrays can yield up to a five-fold increase in responsivity [3]. Therefore, it is again the author's hypothesis that should the discs arrays be positioned close to the $\mathrm{PbSe}-\mathrm{SiO}_{2}$ interface, an enhancement of greater than three-times is achievable over this baseline. This would be due to the enhanced electric field intensity leading to excess carrier generation in the $\mathrm{PbSe}$, which would lead to an effective increase in both absorption and internal quantum 
efficiency. To reiterate, this is a point of further investigation for future study. It should be noted that the second explanation for the absence of the interference dip at $3.4 \mu \mathrm{m}$ is consistent with the assertion that peaks in figure 61 in the vicinity of 3.4-3.6 $\mu \mathrm{m}$ are associated with SPR.

Interestingly the peak responsivity of the sample with Pt discs has a value that is similar to that of the $230 \mathrm{~K}$ spectrum shown in figure 22. Indeed, figure 75 shows a comparison indicating that the detectors with embedded Pt discs have similar sensitivity to the $230 \mathrm{~K}$ spectra in the baseline detectors at the PbSe absorption edge of $4.3 \mu \mathrm{m}$. Moreover, the sensitivity of the embedded disc samples is greater than that of the baseline detectors over a large portion of the MWIR band. With proper fabrication and optimization, the room temperature samples may have even greater sensitivity over the baseline detectors at lower temperature. Though the disc detectors are not optimized, these results still serve as a proof of concept that surface plasmon resonant structures enable room temperature operation $\mathrm{PbSe}$ photoconductors with higher sensitivity.

\subsection{PbSe Devices with Embedded Metallic Back Reflector}

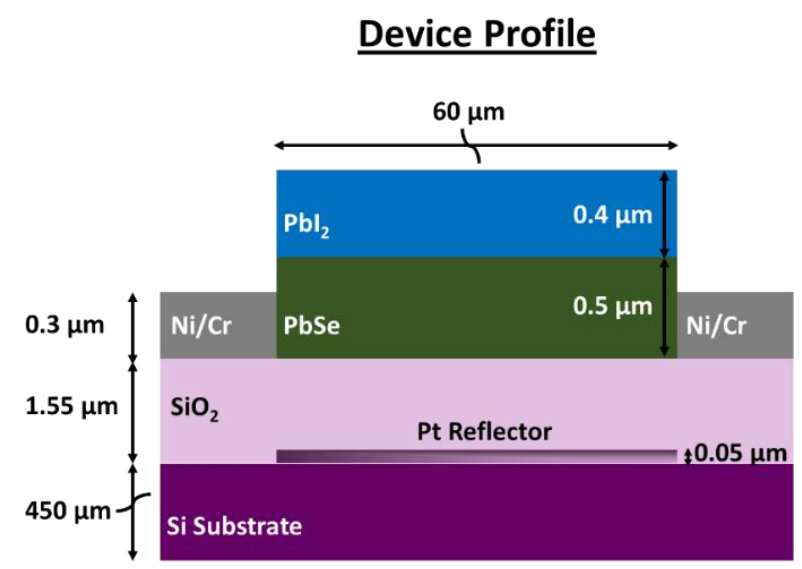

\section{Fabricated Device with \\ Pt Back Reflector}

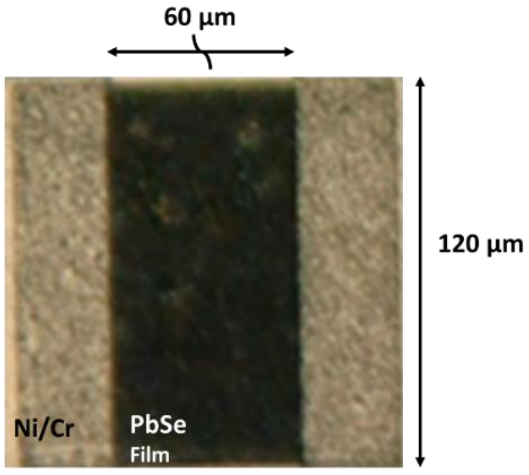

Figure 76. PbSe photoconductive devices with embedded Pt back reflector; (left) depiction of the designed device cross-section, (right) top view of fabricated PbSe device. 
In Chapter 5, a large increase was predicted over the baseline detector for $\mathrm{PbSe}$ devices with an embedded Pt back reflector. To confirm this prediction, PbSe devices with Pt back reflectors were fabricated. Similar to the metallic disc fabrication process, $50 \mathrm{~nm}$ thick Pt back reflectors were deposited on the native $\mathrm{SiO}_{2}$ layer of the $\mathrm{Si}$ substrate. In accordance with the desired resonance discussed in Chapter 5, plasma enhanced chemical vapor deposition was used to grow a $1.5 \mu \mathrm{m} \mathrm{SiO} \mathrm{S}_{2}$ layer utilized as a dielectric spacer. Following this step, the modified baseline process for reduced roughness $\mathrm{PbSe}$ detector fabrication (describe in Appendix A) was followed to fabricate $60 \mu \mathrm{m}$ (width) $\mathrm{x}$ $120 \mu \mathrm{m}$ (length) photoconductive test detectors similar to that of the baseline configuration. Figure 79 depicts the devices with embedded metallic reflectors.

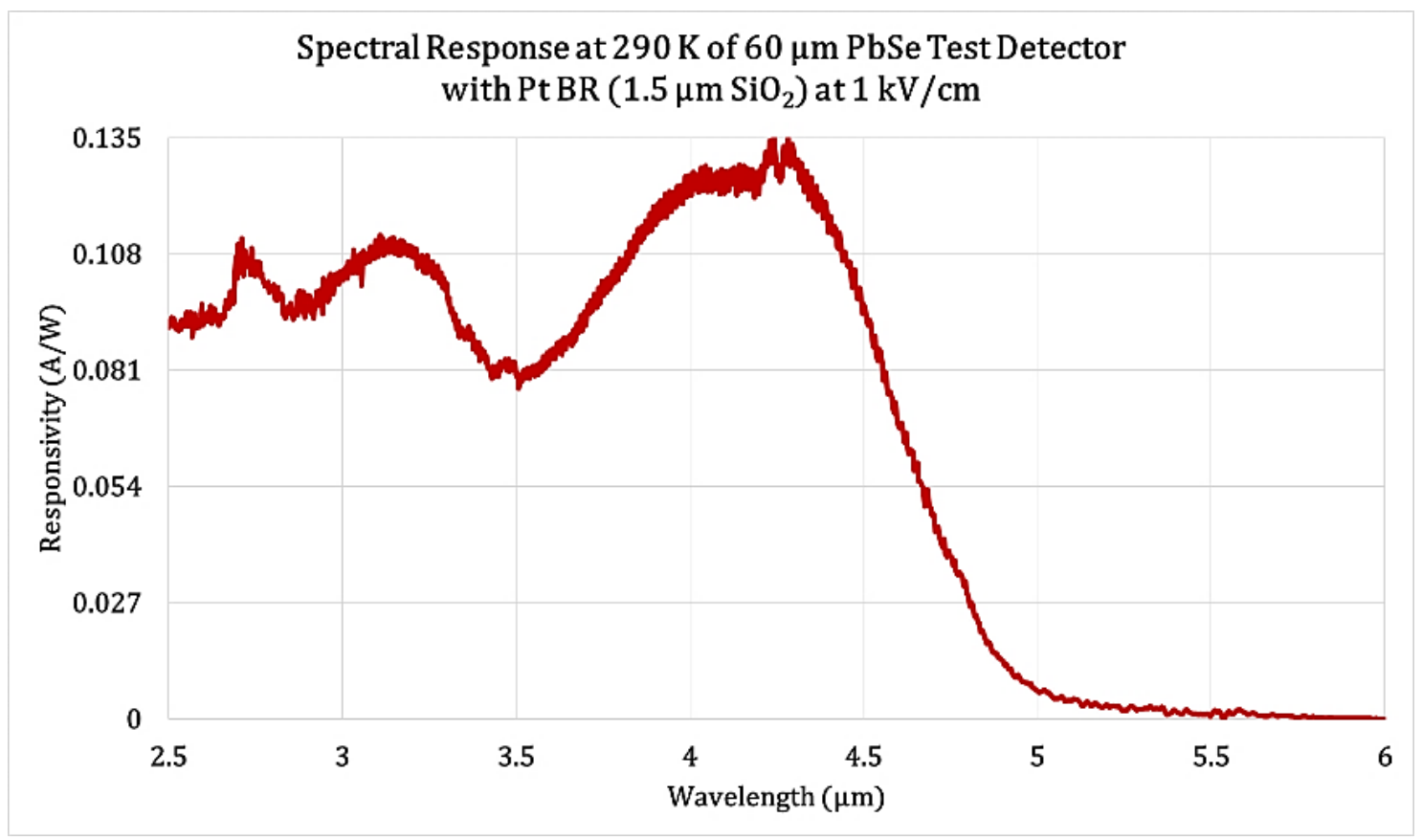

Figure 77. Room temperature (290 K) spectral response of PbSe test detectors with embedded Pt back reflector and $1.5 \mu \mathrm{m} \mathrm{SiO} \mathrm{O}_{2}$ spacer under an applied field of $1 \mathrm{kV} / \mathrm{cm}$. 
Detectors with embedded Pt reflectors were tested using the same spectral response method described above for the embedded disc arrays. A $6 \mathrm{~V}$ bias was applied to the detector, resulting in a $1 \mathrm{kV} / \mathrm{cm}$ electric field. It was found that the spectrum in figure 77 is very similar in shape to that of the predicted spectra in figure 66, which will be discussed in more detail at the end of this chapter. Two peaks from thin film interference were clearly observed at $4.3 \mu \mathrm{m}$ and $3.2 \mu \mathrm{m}$, suggesting a free spectral range of $1.1 \mu \mathrm{m}$. This free spectral range was calculated to be within $1.6 \%$ of the theoretical value for a film with layer thicknesses of $300 \mathrm{~nm}$ for $\mathrm{PbI}_{2}, 500 \mathrm{~nm}$ for $\mathrm{PbSe}, 1500 \mathrm{~nm}$ for $\mathrm{SiO}_{2}$, and $50 \mathrm{~nm}$ for Pt. In this case the substrate was excluded from the calculations, because all of the incident light should either be reflected or dissipated in the Pt layer based on previous calculations. Incidentally, this is also why FTIR absorption measurements were not conducted on these samples.

The amplitude of the peak spectral response shown in figure 77 is approximately 1.7 times less than that of the baseline detectors. However, notice that the applied field across these detectors is three-times less than that of the field applied to the baseline detectors. The photo-generated current $\mathrm{I}_{\mathrm{L}}$ in a photoconductor is directly proportional to the applied electric field $\mathrm{E}$ across the detector by equation 6.1,

$$
I_{L}=J_{L} A=q G_{L} \tau\left(\mu_{n}+\mu_{p}\right) A E
$$

where $\mathrm{J}_{\mathrm{L}}$ is the current density in $\mathrm{A} / \mathrm{cm}^{2}$, $\mathrm{A}$ is the detector area in $\mathrm{cm}^{2}, \mathrm{q}$ is the charge constant, $\mathrm{G}_{\mathrm{L}}$ is the carrier generation rate in $\mathrm{cm}^{-3} \mathrm{~s}^{-}, \tau$ is the carrier recombination lifetime in $\mu \mathrm{s}$, and the mobility of the electrons and holes in $\mathrm{cm}^{2} / \mathrm{Vs}$ is given by $\mu_{n}$ and $\mu_{p}$, respectively [4-5]. The photocurrent scales linearly with applied electric field until the products $\mu_{n} E$ and $\mu_{p} E$ reach their saturation velocities. In baseline detectors, we have 
observed the photocurrent to continually increase with applied fields in excess of 5 $\mathrm{kV} / \mathrm{cm}$, so linearly scaling the data up to $3 \mathrm{kV} / \mathrm{cm}$ to predict higher field performance was considered to be an acceptable approximation.
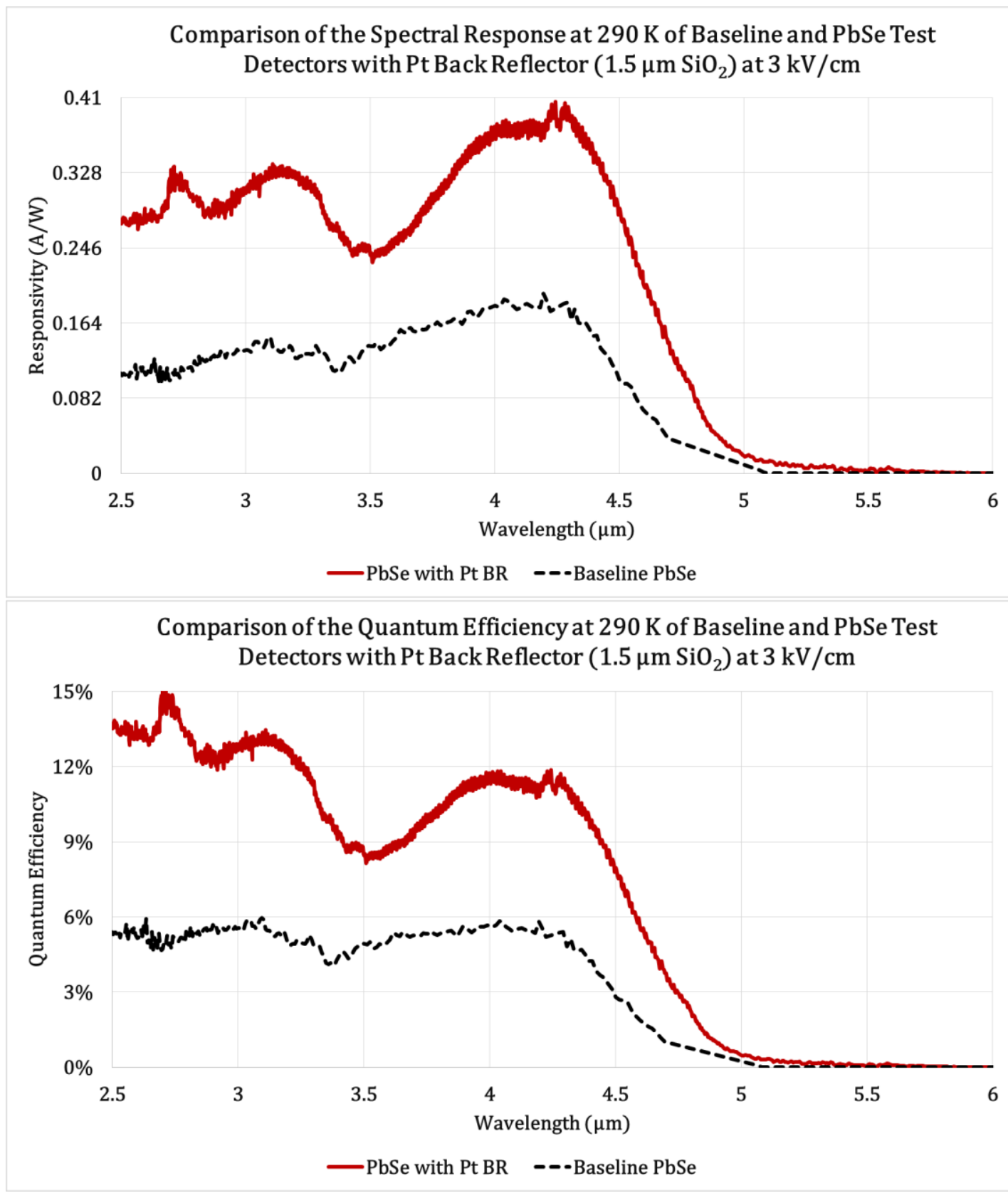

Figure 78. Comparison of room temperature performance of baseline PbSe and predicted performance with Pt back reflector at $3 \mathrm{kV} / \mathrm{cm}$. 
The amplitude of the spectral response spectrum in figure 77 was linearly scaled to predict and directly compare the performance of the test detector with the embedded back reflector to that of the baseline $\mathrm{PbSe}$ detectors at room temperature under an applied field strength of $3 \mathrm{kV} / \mathrm{cm}$. In figure 78, the interference peaks in both the baseline and Pt back reflector test detectors clearly overlap at 3.1 and $4.1 \mu \mathrm{m}$. Indeed, when the same free spectral range calculations were performed on the results from the baseline films the value obtained was $1.13 \mu \mathrm{m}$, which is within $2 \%$ of the distance between peaks in the measured spectra. Considering the similarity between the shapes of the observed responsivity spectra in figure 78 , it may be tempting to assume that the design process described in Chapter 4 and Chapter 5 was unnecessary. After all, a back reflector could just as easily be fabricated in the baseline detector configuration. From the modeled results verified in figure 49, the baseline $\mathrm{PbSe}$ films have a $\mathrm{SiO}_{2}$ layer thickness of 1225 nm. However, consider figure $41 \mathrm{in}$ which the absorption spectrum for different $\mathrm{SiO}_{2}$ thicknesses was presented. For a $1200 \mathrm{~nm} \mathrm{SiO} 2$ thickness, the absorption peaks are closer to the lower end of the spectrum. Discussed in Chapter 5, the responsivity is weighted toward longer wavelengths. Therefore, by designing the back reflector configuration to have a $\mathrm{SiO}_{2}$ thickness of $1500 \mathrm{~nm}$, the detector performance has been optimized for longer wavelengths.

In contrast to the $\mathrm{PbSe}$ alone, the majority of the light that would transmit into the substrate is reflected back for a second pass through the film. As can been seen, under the same electric field, this retro-reflective surface leads to a factor of two enhancement in responsivity over the whole MWIR band. Moreover, an examination of figure 79 shows the scaled sensitivity at $290 \mathrm{~K}$ in the back reflector embedded detector is similar to that of 
the baseline detectors at $250 \mathrm{~K}$. Similar to the disc-embedded detectors discussed above, these results serve as confirmation that the back reflector structure is a viable method for increasing the operating temperature of PbSe photoconductors.

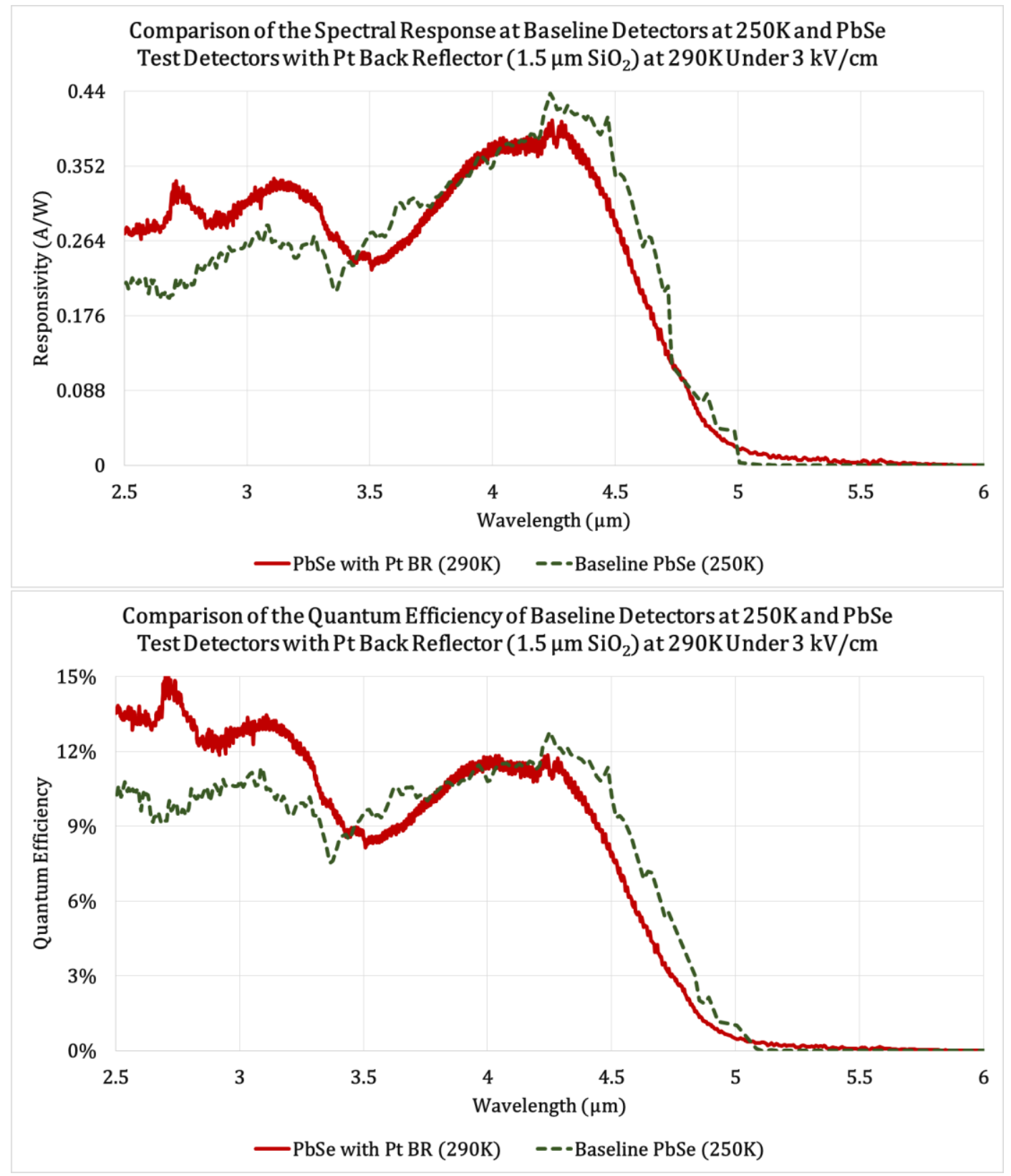

Figure 79. Comparison of $290 \mathrm{~K}$ spectra of PbSe with embedded Pt reflector with that of the $250 \mathrm{~K}$ spectra of baseline detectors. 
It should be noted that part of the enhancement in responsivity and quantum efficiency is likely due to the reduction in roughness, described in Appendix A, from 500 $\mathrm{nm}$ peak-to-peak to $100 \mathrm{~nm}$ peak-to-peak leading to smoother interfaces and less variance in layer thickness. Even with the reduced roughness, however, the spectrum is broadened when compared to the model predictions.

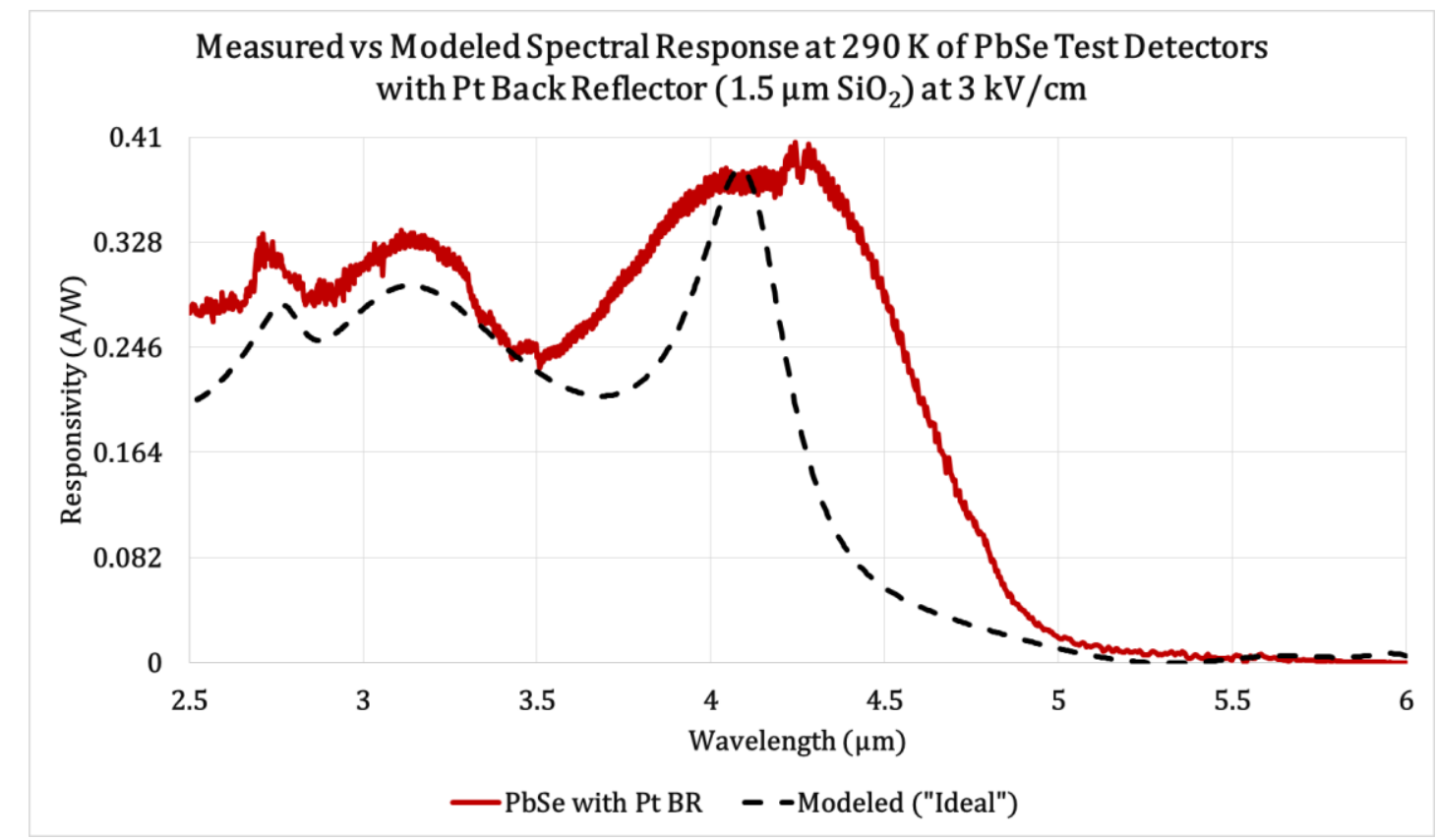

Figure 80. Measured vs modeled spectral response at $290 \mathrm{~K}$ of PbSe test detectors with embedded Pt back reflector and $1.5 \mathrm{\mu m} \mathrm{SiO}_{2}$ layer.

In figure 80 , the measured and modeled spectra are very similar, with their peaks residing in nearly identical locations. The most noticeable difference is that of the width of the primary peak at $4.1 \mu \mathrm{m}$. In the predicted results, the peak is narrow having a FWHM of approximately $230 \mathrm{~nm}$. By comparison, the measured results have a FWHM of $750 \mathrm{~nm}$. This broadening is due to the induced and inherent roughness from the polycrystalline grains in the PbSe films. As broadband light travels through the film, it will be scattered by PbSe crystallites, impurities, etc. This scattering increases the path 
length within the film, leading to increased absorption and altering the angle of incidence for interference fringes throughout various parts of the beam propagating through the film. An increase in absorption explains the slight increase in spectral response over that of the modeled results, whereas the change in interference due to the increase in path length and incident angle is responsible for broadening. Yet, from figure 80 it is apparent that the majority of the photons that contribute to photocurrent travel without deviation from scattering through the film resulting in constructive interference and the primary peak at $4.1 \mu \mathrm{m}$. 


\section{Chapter 6 References}

[1] M. J. Madou, Fundamentals of Microfabrication: The Science of Miniaturization, Second Edition. CRC Press, 2002.

[2] S. A. Campbell, Fabrication Engineering at the Micro- and Nanoscale, Fourth Edition. Oxford University Press, New York, 2013.

[3] X. Tang, et al. "Plasmon resonance enhanced colloidal HgSe quantum dot filterless narrowband photodetectors for mid-wave infrared," J. Mater. Chem. C, $5,2017$.

[4] J. Singh, Optoelectronics: An Introduction to Materials and Devices, McGrawHill Companies, Inc., 1996.

[5] D. A. Neamen, Semiconductor Physics and Devices: Basic Principles, $3^{\text {rd }}$ Ed, New York, NY: McGraw Hill, 2003. 


\section{Chapter 7: Conclusion}

The goal of this study was to increase the performance of $\mathrm{PbSe}$ photoconductive detectors, such that the sensitivity at room temperature is comparable to that of the current stat-of-the-art at lower temperatures. Discussed in Chapter 5, an absorption enhancement of up to three-times over the baseline was predicted for surface plasmon resonant discs, and an absorption enhancement of up to 2.55-times over that of the baseline PbSe absorption was predicted both numerically and by finite element modeling for the back reflector structure. In Chapter 6 it was shown that the surface plasmon resonant discs embedded in $\mathrm{PbSe}$ devices produced a factor of three enhancement in spectral responsivity at room temperature when compared with the baseline detectors under the same applied electric field. Moreover, it was shown that the disc-embedded detectors have performance at room temperature comparable to the baseline detectors at $230 \mathrm{~K}$. These results confirm the original hypothesis that surface plasmon resonant structures can be designed and tailored to increase the operating temperature of $\mathrm{PbSe}$ photoconductive devices. However, the spectra of the measured devices with embedded discs did not resemble the predicted results due to the large $\mathrm{SiO}_{2}$ insulation layer. As a subject of future study, this discrepancy can be better understood and corrected. With proper optimization of the fabrication process, even greater room-temperature sensitivity could be achievable.

Also discussed in Chapter 6, the measured responsivity and quantum efficiency of the $\mathrm{PbSe}$ devices with embedded back reflector showed remarkable agreement with modeled predictions, and a factor of two sensitivity enhancement over the baseline $\mathrm{PbSe}$ detectors. Furthermore, it was shown that the back reflector structure lead to performance 
at $290 \mathrm{~K}$ that is similar to the baseline detector at $250 \mathrm{~K}$, thereby confirming the viability of the back reflector interference structure as a mechanism for raising the operating temperature of PbSe photoconductors from $250 \mathrm{~K}$ to 290K. Presented in Chapter 5, FEM predictions demonstrate that the resonance can be tuned to specific wavelengths and regions by changing the thickness of the underlying $\mathrm{SiO}_{2}$ layer. Indeed, this opens up the possibility for tunable room temperature, low cost infrared sensing devices with excellent performance.

Table 12. Unit cell parameters for modeling the combination of surface plasmon resonant Pt discs and Pt back reflector in PbSe films.

\begin{tabular}{|c|c|c|}
\hline Layer/Structure & Thickness $(\mathbf{n m})$ & Pitch* $\left.^{*} \mathbf{n m}\right)$ \\
\hline $\mathrm{PbI}_{2}$ & 300 & 1000 \\
\hline $\mathrm{PbSe}$ & 500 & 1000 \\
\hline $\begin{array}{c}\text { Pt Disc } \\
\text { (PEC Boundary Conditions) }\end{array}$ & 40 & $\begin{array}{c}500 \\
\text { (*Diameter) }\end{array}$ \\
\hline $\mathrm{SiO}_{2}$ & 1500 & 1000 \\
\hline $\mathrm{Pt}$ back reflector & 50 & 1000 \\
\hline $\mathrm{Si}$ & 50 & 1000 \\
\hline
\end{tabular}

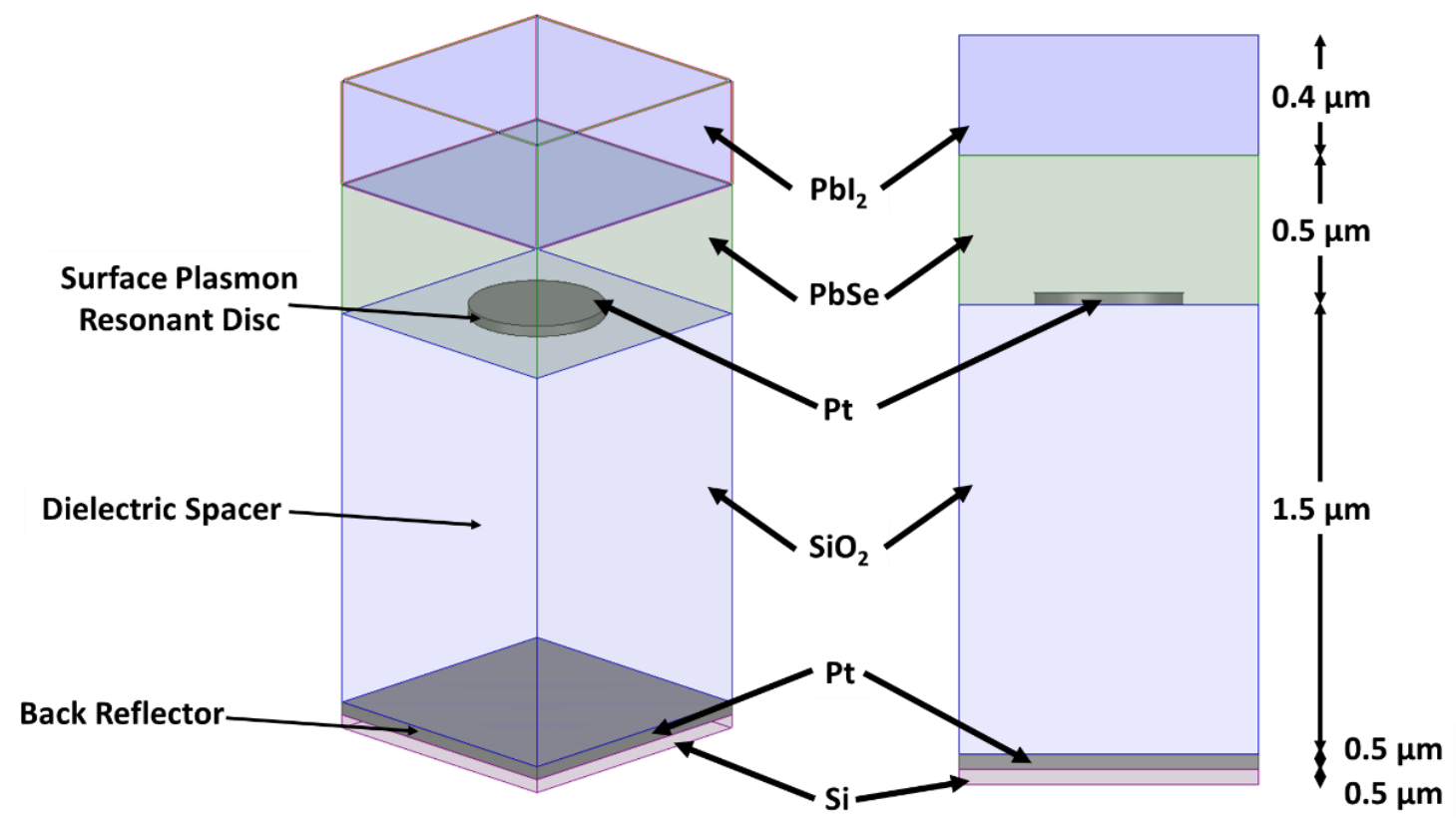

Figure 81. Unit cell of PbSe film with combination of surface plasmon resonant Pt discs and Pt back reflector modeled in ANSYS HFSS. 
My projections suggest that the back reflector and surface plasmon resonant structures can be combined for even greater enhancement. In fact this configuration has been demonstrated by Tang in $\mathrm{HgTe}$ colloidal quantum dot photovoltaic detectors, yielding excellent MWIR imaging capability at cryogenic temperatures [1]. Recall from Chapter 2 that I measured an absorption of 30-35\% in the baseline PbSe films. With both the plasmonic discs and the back reflector structure, I observed by analysis that this absorption can be increased by levels between three and seven times in narrow spectral regions within the MWIR regime. Films were modeled in ANSYS HFSS with unit cell parameters shown in table 12. The unit cell is shown in figure 81 .

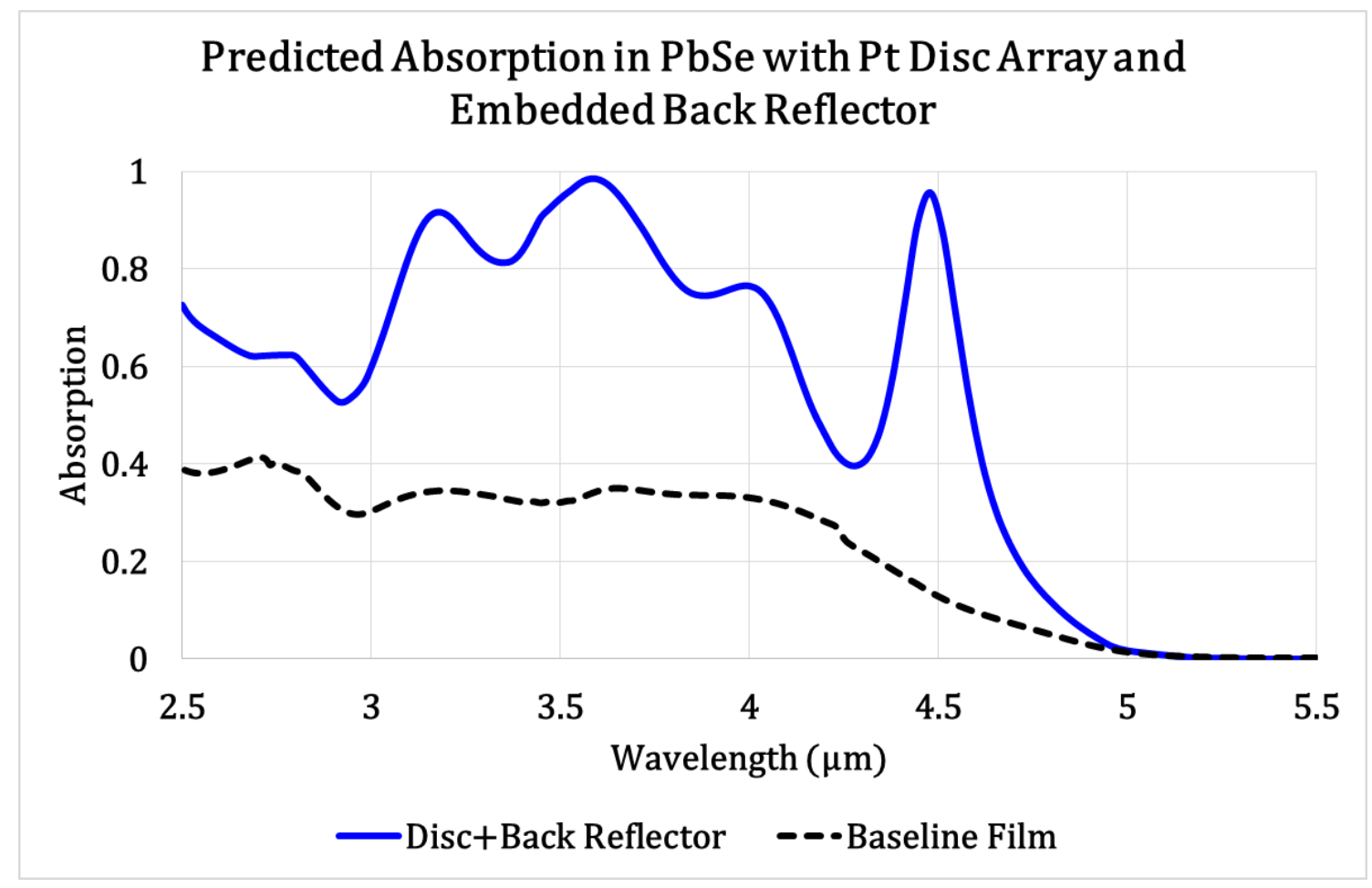

Figure 82. Predicted absorption in PbSe films with combined optical enhancement techniques of SPR and embedded metallic back reflector compared to the modeled absorption in baseline films.

Consider the spectra of figure 82 , which show the predicted absorption as a result of combining a Pt disc array with a Pt back reflector in PbSe films, as well as the 
modeled baseline absorption at room temperature. The absorption results predict a broadband enhancement over the whole $3 \mu \mathrm{m}-5 \mu \mathrm{m}$ band, with maximums reaching nearly $100 \%$ at $3.6 \mu \mathrm{m}$ and $4.5 \mu \mathrm{m}$. Further, the disc array is conveniently designed such that there is a decrease in absorption in the $\mathrm{CO}_{2}$ atmospheric window between $4.1 \mu \mathrm{m}$ and $4.4 \mu \mathrm{m}$. This small region also corresponds to the absorption edge of $\mathrm{PbSe}(\sim 4.3$ $\mu \mathrm{m})$, so it is the author's hypothesis that the steep reduction in absorption at $4.3 \mu \mathrm{m}$ is due to the steep drop in absorption below the bandgap energy. The sharp increase absorption shortly after $4.3 \mu \mathrm{m}$ leading the peak at $4.5 \mu \mathrm{m}$ is due to the large field intensity produced from both surface plasmon resonance and interference. This large field intensity warrants further discussion.

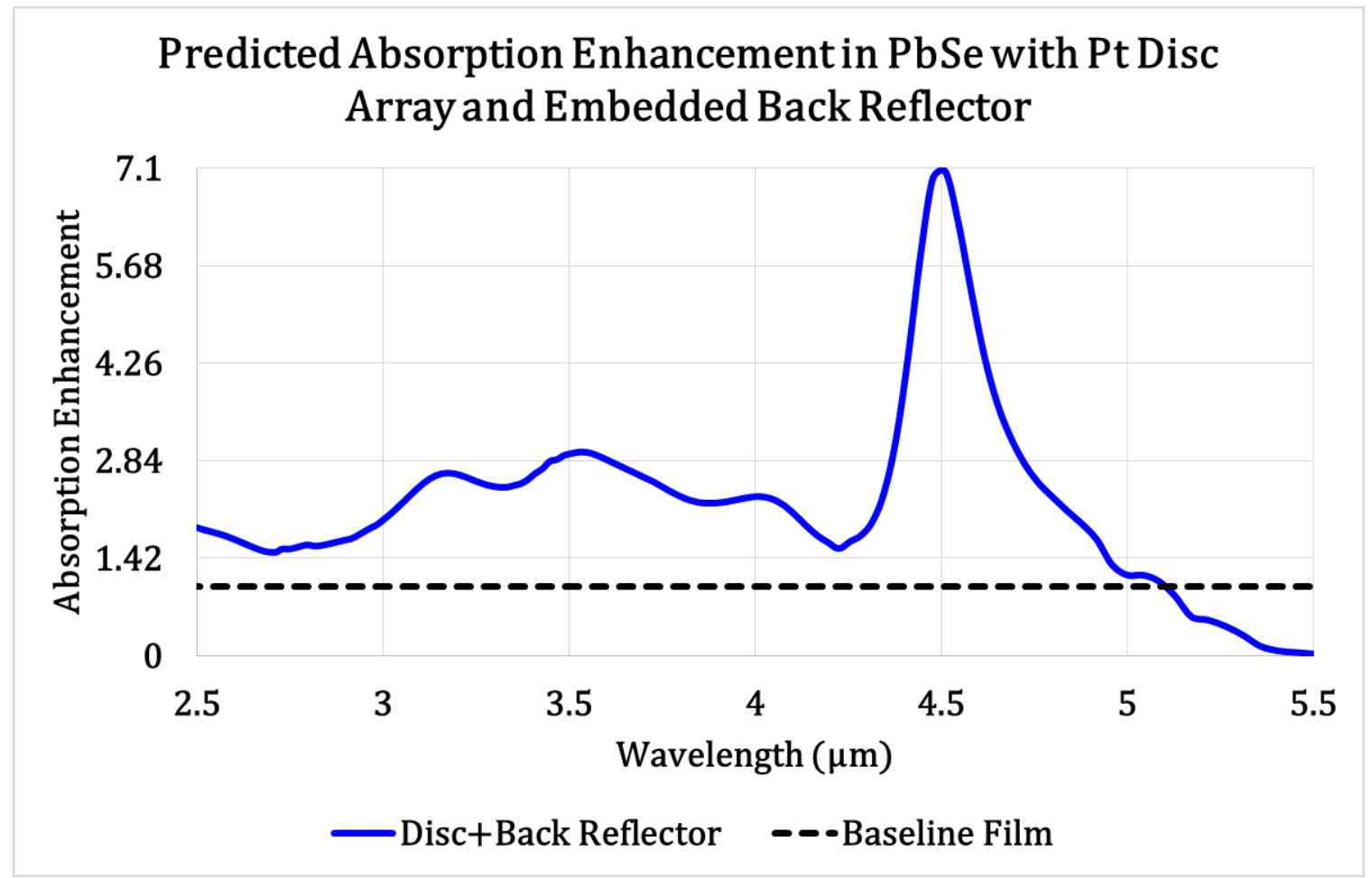

Figure 83. Predicted absorption enhancement in PbSe films with combined optical enhancement techniques of SPR and embedded metallic back reflector compared to the modeled absorption in baseline films. 
However, before moving on consider figure 83, which shows the predicted absorption enhancement, defined as the absorption of the resonant structure enhanced film normalized to the baseline absorption spectrum, gained by combining surface plasmon resonant disc arrays with the back reflector structure. Compared to the baseline spectrum, the combination of the disc array and back reflector structures yield greater than 1.5-times the absorption over the whole band, three-times the absorption at approximately $3.6 \mu \mathrm{m}$, and over a factor of seven times greater absorption at $4.48 \mu \mathrm{m}$.

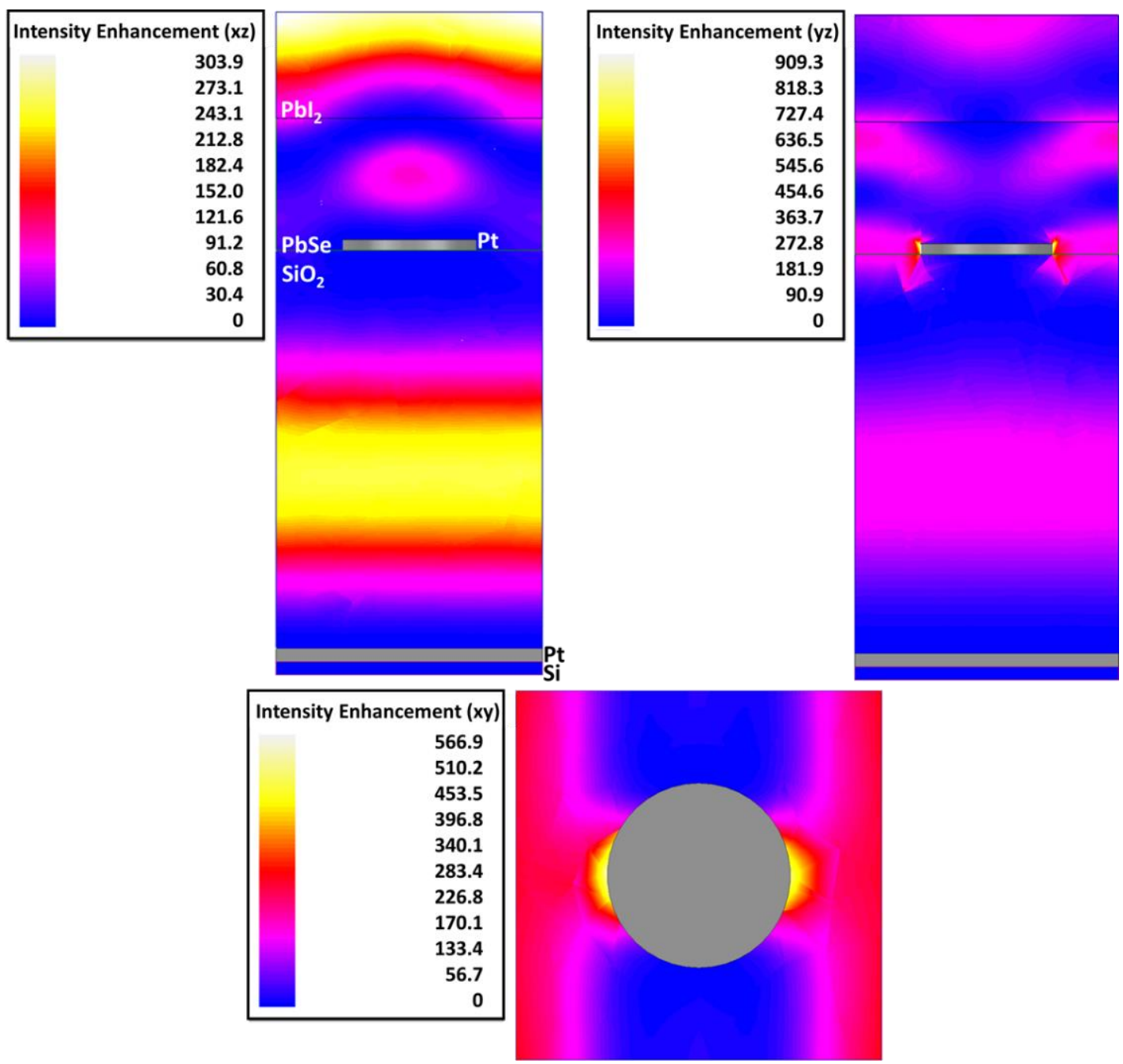

Figure 84. Electric field Intensity enhancement plots for surface plasmon resonant Pt discs and Pt back reflector embedded in PbSe films at an incident wavelength of $3.60 \mu \mathrm{m}$. in the (top-left) xz-, (top-right) yz-, and (bottom) xy-directions. 


\begin{tabular}{|r|}
\hline Intensity Enhancement (xz) \\
701.9 \\
631.7 \\
561.5 \\
491.3 \\
421.1 \\
350.9 \\
280.8 \\
210.6 \\
140.4 \\
70.2 \\
0 \\
\hline
\end{tabular}
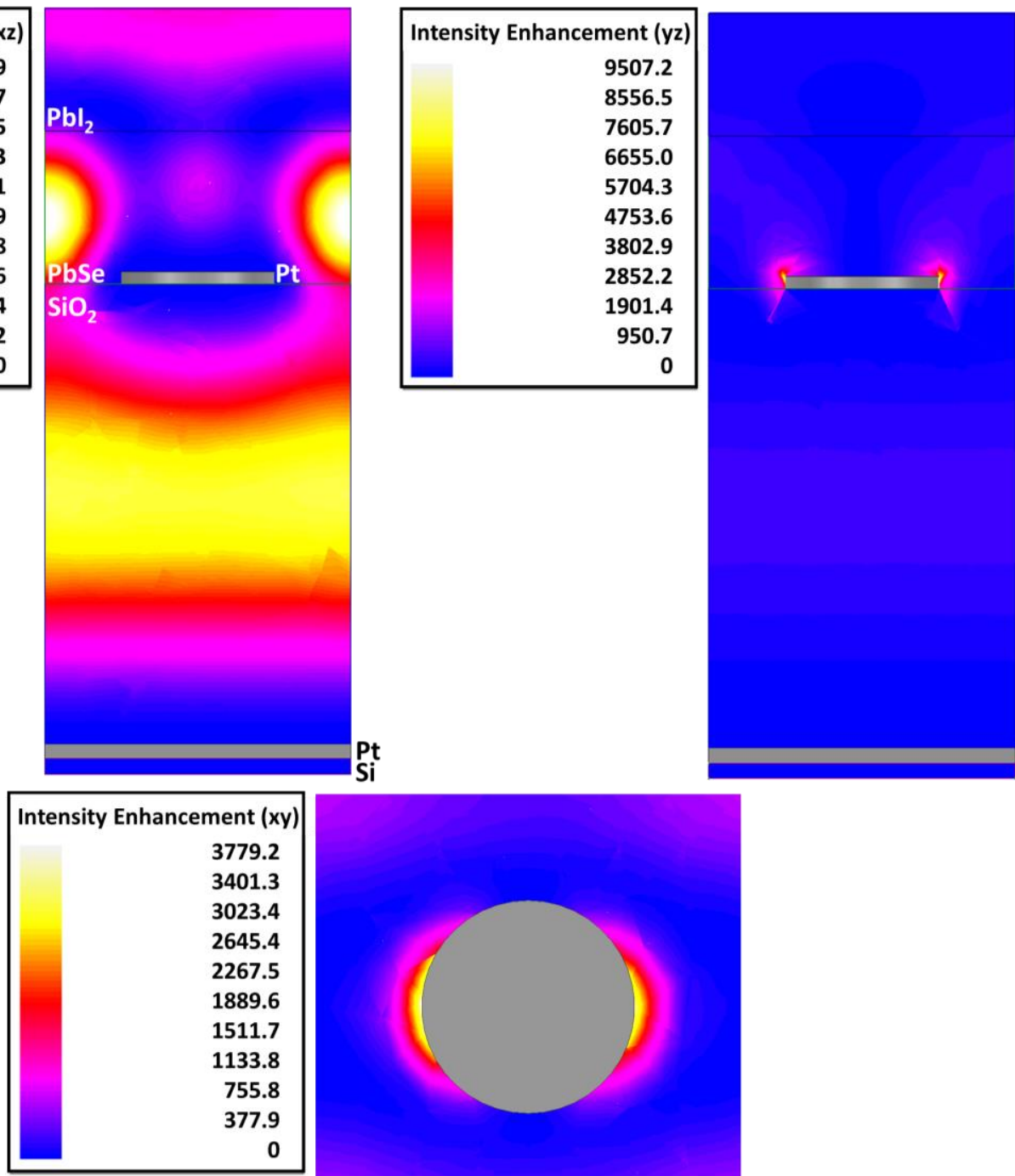

$\mathrm{Pi}_{\mathrm{Si}}$

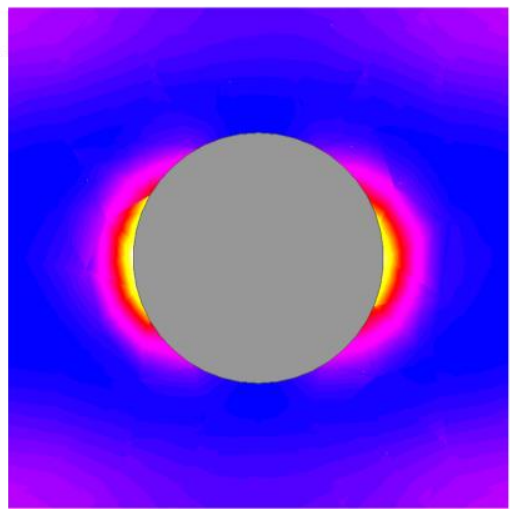

Figure 85. Electric field intensity enhancement plots for surface plasmon resonant Pt discs and Pt back reflector embedded in PbSe films at an incident wavelength of $4.48 \mu \mathrm{m}$. in the (top-left) xz-, (top-right) yz-, and (bottom) xy-directions.

To observe the origins of this large absorption increase and gain insight into the increase in electric field intensity offered by this configuration, discrete frequency calculations were performed using ANSYS HFSS. Figure 84 and figure 85 show the electric field intensity enhancement, defined as the resulting electric field intensity in the film normalized to the incident electric field intensity, in the PbSe film with an embedded Pt disc array and Pt back reflector for an incident plane wave with free a space 
wavelength of $3.6 \mu \mathrm{m}$ and $4.48 \mu \mathrm{m}$, respectively. Nearly $100 \%$ absorption is predicted at these wavelengths, so they were selected for further analysis. It should be noted, however, that large intensity enhancements are also expected at $3.2 \mu \mathrm{m}$ and $4 \mu \mathrm{m}$. In the xz-plane of figure 84 (top-left), the majority of the intensity enhancement is in the $\mathrm{SiO}_{2}$ layer, suggesting the Pt disc and Pt back reflector make a plane-parallel (Fabry-Perot) cavity [2-5]. Still, there is an intensity enhancement between 60 and 90 in the middle of the PbSe layer. In the xy-plane (bottom), the effects of both surface plasmon resonance and interference are observed. The field intensity in the vicinity of the disc surface is similar to the intensity shown in Chapter 4 for surface plasmon resonant discs. The intensity observed on the edges of the unit cell in the xy-plane are those associated with interference. To be sure, the fields in the yz-plane (top-right) show the large electric field intensity enhancement expected around the disc, as well as the fringes expected of interference. With a field intensity enhancement of over 900 possible using this configuration, it is clear why the absorption is predicted to be nearly $100 \%$.

Similar phenomena are observed in figure 85 . The intensity enhancement at 4.48 $\mu \mathrm{m}$ is much larger than at $3.6 \mu \mathrm{m}$. In the xz-plane (top-left), the larger electric field intensity enhancement is again seen in the $\mathrm{SiO}_{2}$ layer, suggesting the same resonant cavity is produced between the Pt discs and Pt back reflector. The field intensity in the $\mathrm{PbSe}$, however, is much larger reaching the maximum intensity enhancement in the space between discs. This is indicative of the dual-dipole effect described in Chapter 3. In the xy-plane (bottom), surface plasmon resonance is again observed. The interference fringes observed in the xy-plane of figure 84 are absent, suggesting that the dominant enhancement mechanism at $4.48 \mu \mathrm{m}$ is surface plasmon resonance. This seems to be 
confirmed in the yz-plane (top-right) of figure 85, with nearly all the field enhancement residing in the near-field of the Pt disc. It would seem that by combining the two techniques, the surface plasmon resonance wavelength has been shifted to $4.48 \mu \mathrm{m}$. An alternative explanation, that seems in part to be the case, is that multipole resonances are being excited within the Pt disc leading to multiple peaks from surface plasmon resonance. This phenomenon warrants further study in the future.

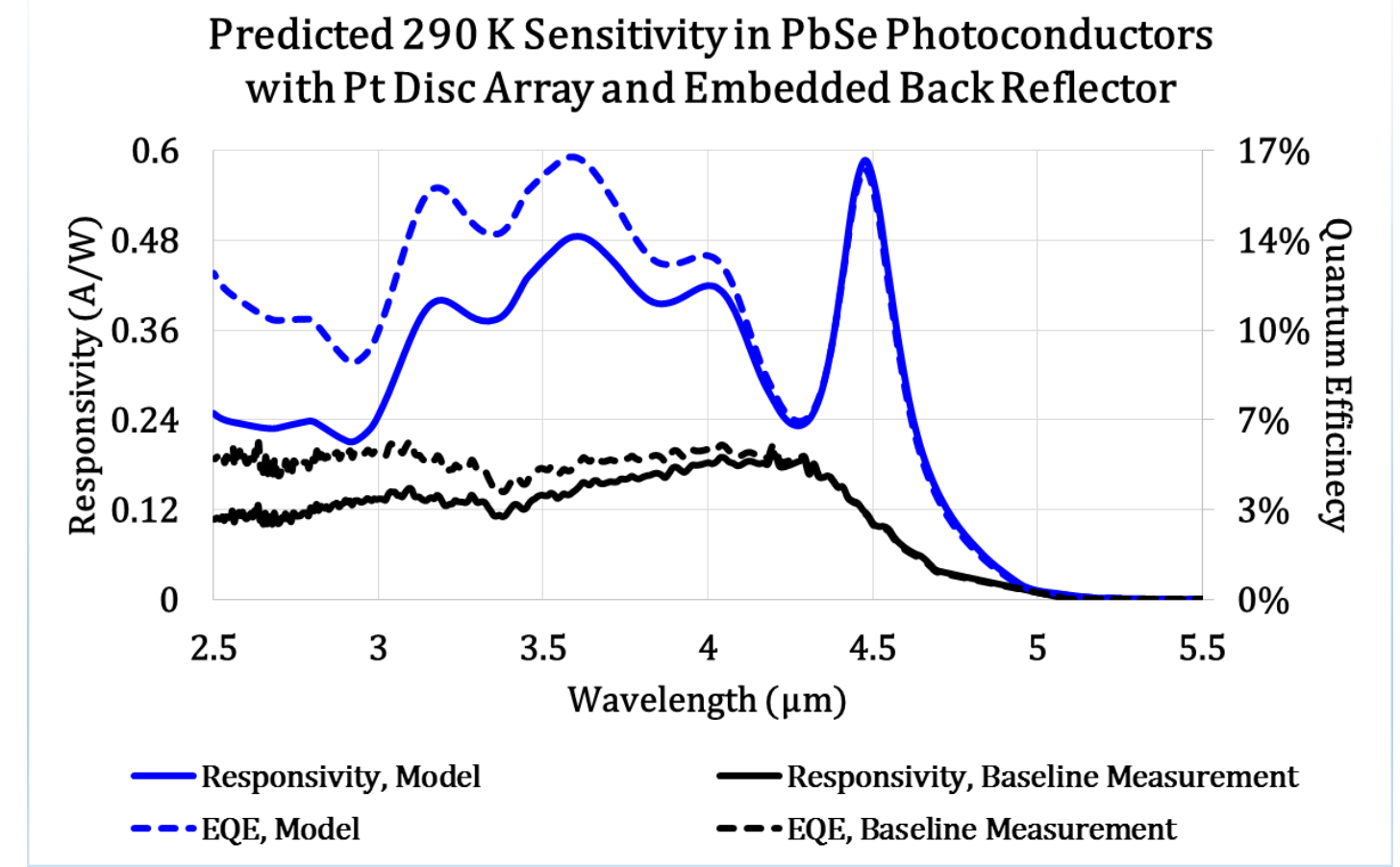

Figure 86. Predicted room temperature spectral responsivity (solid) and quantum efficiency (dashed) of PbSe devices with SPR disc arrays and embedded back reflector compared to the current baseline detector performance.

The large difference between the amplitude of the field intensity enhancement at $3.6 \mu \mathrm{m}$ and $4.48 \mu \mathrm{m}$ is of note, especially in the xy- and yz-planes. The field intensity enhancement for illumination of $4.48 \mu \mathrm{m}$ is $\sim 100$-times greater than that at $3.6 \mu \mathrm{m}$, yet the absorption at the former is slightly lower than the latter. An examination of the 
baseline spectrum in figure 82 shows that the PbSe absorption falls off above the cutoff wavelength. Therefore, it is necessary for the field intensity enhancement to be much larger in this region to achieve such a large absorption. Future investigation into this configuration could yield interesting new avenues for PbSe detectors, such as near room temperature long-wave detection.

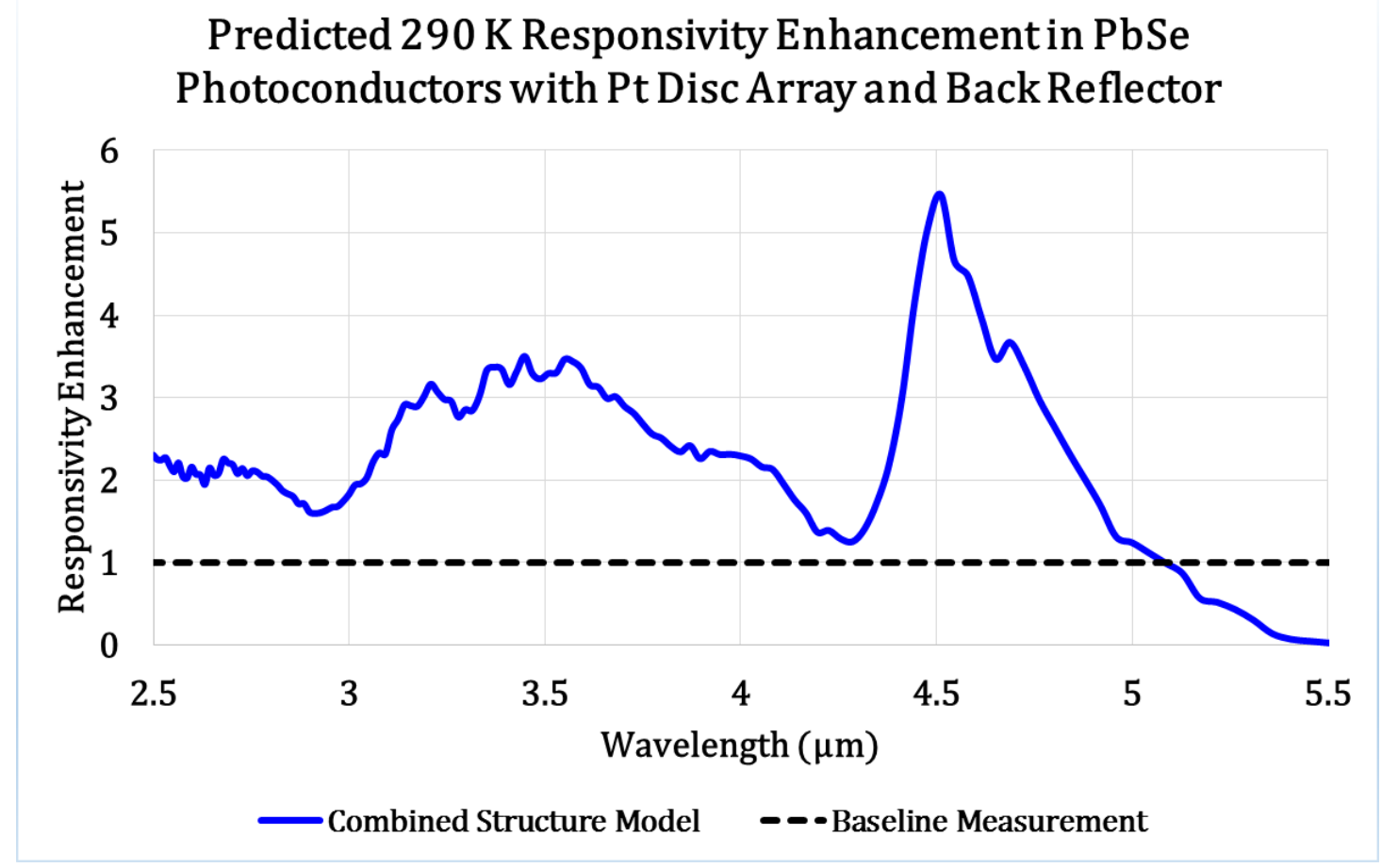

Figure 87. Predicted room temperature spectral responsivity enhancement PbSe devices with SPR disc arrays and embedded back reflector (blue, solid) over the baseline detector performance (black, dashed).

The sensitivity of ideal detectors with these combined techniques has also been predicted by assuming the internal quantum efficiency of the baseline detectors at room temperature $(\sim 17 \%)$ still holds. Presented in figure 86 , the responsivity (blue, solid trace) of the resonant structure enhanced detectors is predicted to reach a maximum of 0.58 A/W at $4.5 \mu \mathrm{m}$, with $0.41 \mathrm{~A} / \mathrm{W}, 0.48 \mathrm{~A} / \mathrm{W}$, and $0.4 \mathrm{~A} / \mathrm{W}$ coinciding with peaks at $4 \mu \mathrm{m}$, $3.6 \mu \mathrm{m}$, and $3.2 \mu \mathrm{m}$, respectively. The quantum efficiency (blue, dashed trace) reaches a 
high of $\sim 17 \%$ at $3.6 \mu \mathrm{m}$ and $4.5 \mu \mathrm{m}$, with greater than $10 \%$ over most of the rest of the MWIR band for the same detector.

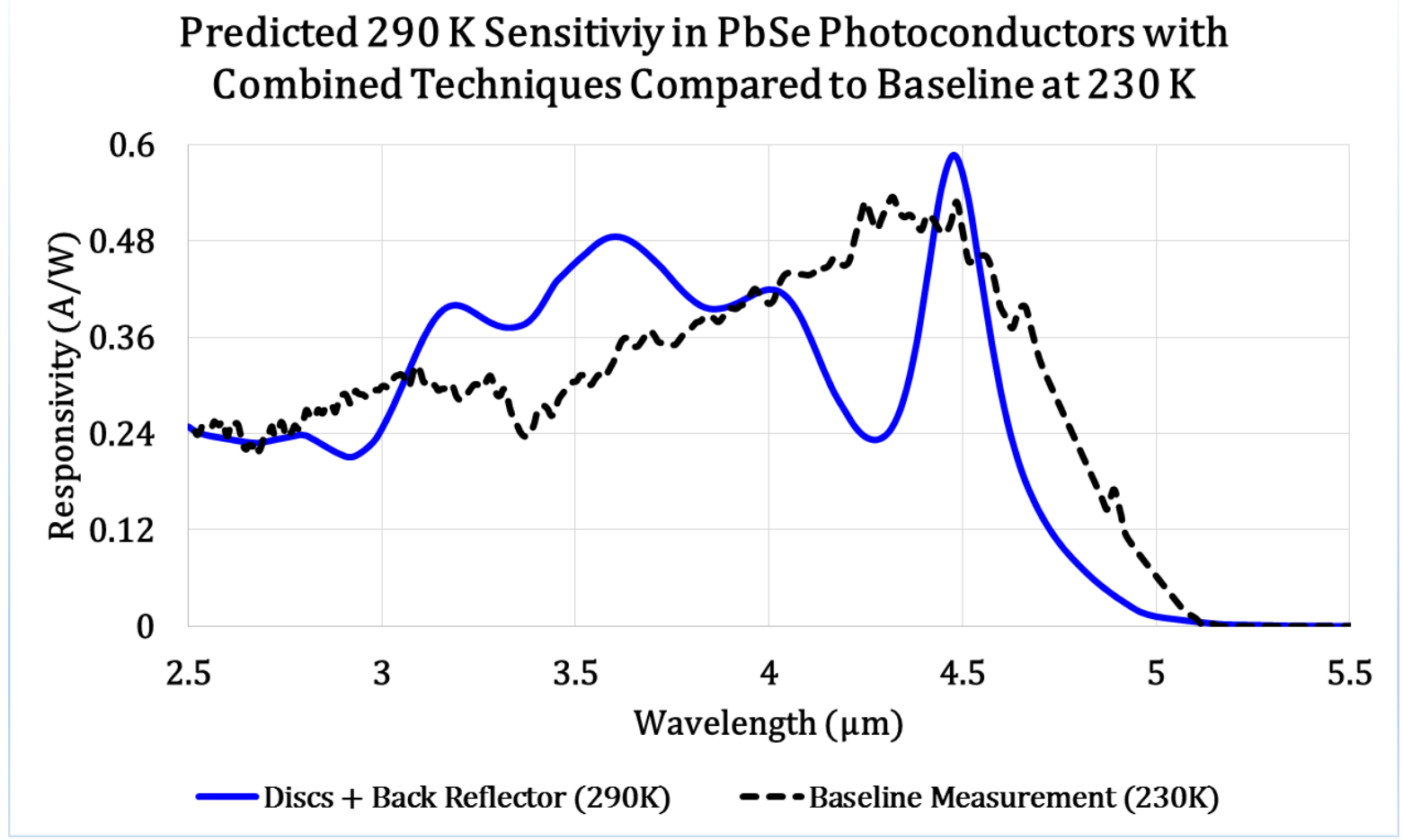

Figure 88. Predicted room temperature (290 K) sensitivity of PbSe detectors with combined Pt disc array and Pt back reflector compared to measured baseline sensitivity at $230 \mathrm{~K}$.

Of note is the fact that the predicted performance at room temperature for this combination of structures is greater than 3-times that of the baseline detector performance at $3.6 \mu \mathrm{m}$ and over 5-times greater at $4.48 \mu \mathrm{m}$. In fact, figure 87 shows that the responsivity is above that of the baseline detector over the whole $3-5 \mu \mathrm{m}$ band.

Moreover, the predicted performance, shown in figure 88, is greater than that of the measured baseline at $230 \mathrm{~K}$ for a majority of the usable MWIR spectrum. These results, along with those shown in Chapter 6, show that by proper design and fabrication of these optical management schemes, the sensitivity of PbSe MWIR detectors can exceed that of 
the current state-of-the-art such that their performance is greater at room temperature than devices with $\mathrm{PbSe}$ alone at temperatures as low as $230 \mathrm{~K}$. 


\section{Chapter 7 References}

[1] X. Tang, M. Ackerman, and P. Guyot-Sionnest, "Thermal Imaging with Plasmon Enhanced HgTe Collidal Quantum Dots Photovoltaic Devices," manuscript nn2018-03871g submitted to ACS Nano, 2018.

[2] E. Hecht, Optics, 4th Edition. Addison-Wesley, New York, 2002.

[3] R. E. Fischer, et al. Optical System Design, 2nd Edition, SPIE Press, McGraw Hill, 2008.

[4] OP-Tec: National Center for Optics and Photonics Education, Fundamental of Light and Lasers, 2nd ed. Texas: Op-TEC, 2013.

[5] W. T. Silfvast, Laser Fundamentals, New York, NY: Cambridge University Press, 2000.

[6] C. C. Davis, Lasers and Electro-Optics: Fundamentals and Engineering, $2^{\text {nd }}$ ed. New York, NY: Cambridge University Press, 2014. 


\section{Appendix A: Fabrication of PbSe Devices}

\section{PbSe Baseline Detector Fabrication}

The deposition process starts with a three-inch single side polished $<100>\mathrm{Si}$ wafer from Pure Wafer, a WRS Company [1] with a $1-1.5 \mu \mathrm{m}$ thick $\mathrm{SiO}_{2}$ layer grown by plasma enhanced chemical vapor deposition (PECVD) on top [2]. Reactive ion etching (RIE) was performed on the $\mathrm{SiO}_{2}$ layer with tetraflouromethane $\left(\mathrm{CF}_{4}\right)$ gas to produce a peak-to-peak roughness of $400-500 \mathrm{~nm}(\sim 177 \mathrm{~nm}$ RMS). Described briefly in Chapter 2, we used a chemical bath deposition process to deposit and sensitize PbSe films on the roughened substrates. The process starts by mixing lead acetate trihydrate $\left(\mathrm{Pb}\left(\mathrm{C}_{2} \mathrm{H}_{3} \mathrm{O}_{2}\right)_{2}\right)$ and optical grade selenourea $\left(\left(\mathrm{H}_{2} \mathrm{~N}\right)_{2} \mathrm{C}=\mathrm{Se}\right)$ in a chemical bath to produce a $\sim 1 \mu \mathrm{m}$ thick PbSe film. Films were then introduced into an oxygen furnace at $\sim 450{ }^{\circ} \mathrm{C}$ for oxidation, followed by a halogen furnace at $350{ }^{\circ} \mathrm{C}$ to complete sensitization.

An AZ 1518 photoresist from Microchemicals Gmbh with a thickness of $4 \mu \mathrm{m}$ was spin coated at a rate of $4000 \mathrm{rpm}$ onto the surface for 40 seconds [4]. After a 60 second prebake at $110^{\circ} \mathrm{C}$, the sample was then put into a Karl Suss MA6 Mid/Deep UV mask aligner under a photomask designed for PbSe test detectors [5]. Upon UV illumination with a wavelength of $350 \mathrm{~nm}$, bonds between the molecules of the resist in the exposed area become weaker, thereby making them soluble in developer [2-3]. The sample was then immersed in a 1:1 solution of general purpose AZ developer and DI water to remove the exposed areas, exposing the $\mathrm{PbI}_{2}$ layer of the $\mathrm{PbSe}$ film in a process called etch-back. The exposed $\mathrm{PbI}_{2}$ areas were then removed down to the PbSe layer by RIE, an anisotropic technique, defining the areas for metal contact deposition. This process also 
defines the detector configuration. The samples were then placed in a bath of acetone to remove the photoresist.
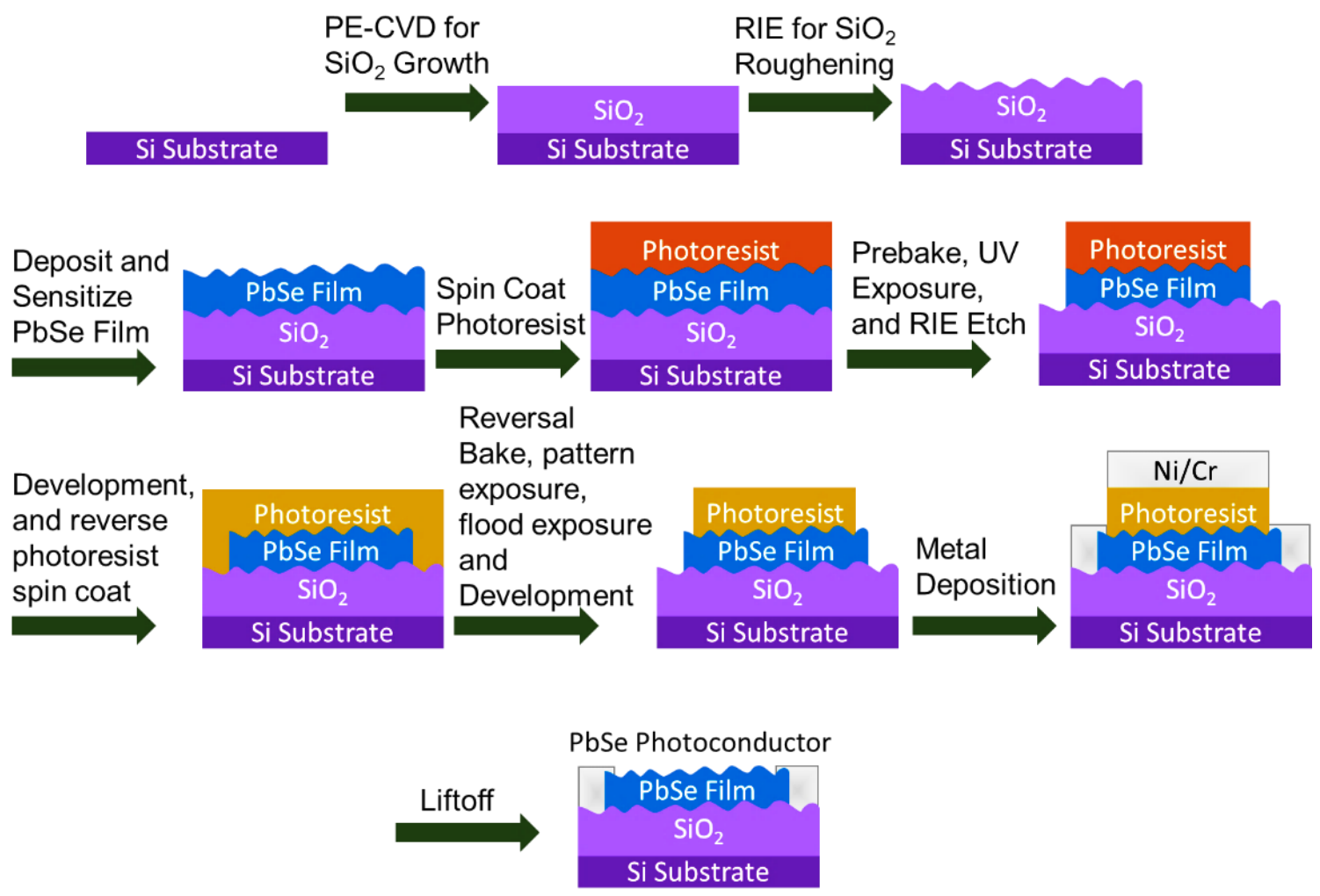

Figure 89. Fabrication process for PbSe photoconductive devices.

After cleaning with IPA and spray drying with $\mathrm{N}_{2}$, AZ 5214-E was spin coated onto the sample to a thickness of $1.6 \mu \mathrm{m}$ at $3000 \mathrm{RPM}$. This compound is a special positive photoresist from Microchemicals Gmbh which works on an image reversal process that results in the same profile as a negative resist [6]. Negative resists are typically used for metal liftoff, a process to remove excess metal after deposition. In a similar process to that described in the previous step, the photoresist was baked at $110{ }^{\circ} \mathrm{C}$ for 80 seconds, exposed and developed [7]. After developing, a second flood exposure was performed to weaken the bonds in the leftover resist. A $15 \mathrm{~nm}$ thick $\mathrm{Cr}$ adhesion layer followed by a $150 \mathrm{~nm}$ layer of Ni was RF sputtered onto the sample to form metal contacts. The sample 
was then placed in AZ developer for liftoff. After drying the fabrication process was complete, resulting in baseline $\mathrm{PbSe}$ detectors. The process for depositing nanodiscs or back reflectors structures described in Chapter 6 is almost identical, with the exception that the metallic structures are patterned and deposited onto the $\mathrm{SiO}_{2}$ layer before $\mathrm{PbSe}$ deposition. Roughening was then performed around the metallic structures for $\mathrm{PbSe}$ adhesion to the substrate. A depiction of the baseline PbSe fabrication process is shown in figure 89.

\section{Preparation for Resonant Structures: PbSe Roughness Reduction}

As discussed in Chapter 2, the baseline PbSe films have a peak-to-peak roughness of approximately $500 \mathrm{~nm}$. Surface plasmon modes exist along the metal-dielectric interface, and, in the case of traveling waves, propagate along the metal surface. Due to the fact that these modes are so tightly coupled to the interface, surface roughness of the metal, dielectric, or both can significantly reduce the propagation distance of SPP into the PbSe thereby reducing the enhancement in the films. Indeed, Fadakar and colleagues demonstrated through finite element analysis that the attenuation in SPP modes perpendicular to the interface increases with the surface roughness of the host material [8]. Moreover, surface roughness has an effect on the back reflector as well, due to the localized variances in refractive index and layer thickness. Therefore, we developed a procedure to reduce the roughness of our PbSe films.

Nanosphere lithography is a patterning technique that does not require the exposure of an electro- or photosensitive film for patterning. Instead, in this process nanometer-scale polystyrene spheres are deposited onto the substrate by either the Langmuir-Blodgett method, or through spin coating [9-13]. For the reduced roughening 
process, the spin coating method described in [10-11] was used. The process starts by mixing $40 \mathrm{~mL}$ of methanol $\left(\mathrm{CH}_{3} \mathrm{OH}\right)$ with $100 \mu \mathrm{L}$ of Triton $\mathrm{X}-100$ surfactant $\left(\mathrm{C}_{14} \mathrm{H}_{22}\right.$ $\left.\mathrm{O}\left(\mathrm{C}_{2} \mathrm{H}_{4} \mathrm{O}\right)_{9} \mathrm{OH}\right)$ to produce a 1:400 solution [10]. $750 \mu \mathrm{L}$ of $1 \mathrm{mg} / \mathrm{mL}$ polystyrene spheres obtained from ThermoFisher Scientific and DI water solution were then added to the mixture [14]. The spheres used for nanoroughening were between $300 \mathrm{~nm}$ and 350 $\mathrm{nm}$ in diameter. The solution was mixed for 10 min by sonication. Si wafers with $\mathrm{SiO}_{2}$ grown by plasma enhanced chemical vapor deposition (PECVD) were then cleaned using isopropyl alcohol, acetone, and DI water before being dried with $\mathrm{N}_{2}$. To further reduce surface contaminants and remove any residue from previous cleaning, the substrates were placed in an $\mathrm{O}_{2}$ plasma cleaner for $10 \mathrm{~min} .100 \mu \mathrm{L}$ of solution was then pipetted and spin coated at a rate of $1500 \mathrm{rpm}$ onto the substrate for 60 seconds. After the solvent had dried, a $\mathrm{CF}_{4}$ etch was performed on the samples for 200 seconds to roughen the substrate around the spheres. Nanospheres were then removed using a combination of sonication in acetone and methanol for 10 min each, followed by an IPA rinse for the final cleaning.

Figure 90 shows a 5000x SEM image of the polystyrene spheres on the $\mathrm{SiO}_{2}$ surface after spin coating. Ideally, the nanospheres would be assembled into a continuous periodic monolayer, however over a $7.62 \mathrm{~cm}$ diameter substrate this proved difficult, as large areas were left uncovered. Nevertheless, we empirically concluded that this new roughening process was adequate for $\mathrm{PbSe}$ adhesion to the substrate. To determine the reduction in roughness in $\mathrm{PbSe}$ films deposited after the new nanoroughening process compared to the baseline process, we measured the surface roughness via atomic force microscopy (AFM) [15]. Figure 91 contains surface roughness maps obtained over a 50 $\mu \mathrm{m}$ area of smooth $\mathrm{SiO}_{2}$ (top-left), baseline roughened $\mathrm{SiO}_{2}$ (top-right), and nanosphere 
lithography roughened (bottom) $\mathrm{SiO}_{2}$ substrates. Notice the difference in uniformity between the baseline roughened and nanosphere lithography roughened maps.

Furthermore, figure 92 shows a five-fold reduction in peak-to-peak roughness from 500 nm peak-to-peak down to $100 \mathrm{~nm}$ peak-to-peak when comparing the baseline to the nanosphere roughened films. This reduction in roughness would prove critical to maximizing the enhancement in the $\mathrm{PbSe}$ films due to resonant structures.

\section{SEM Image after Nanosphere Deposition Process}

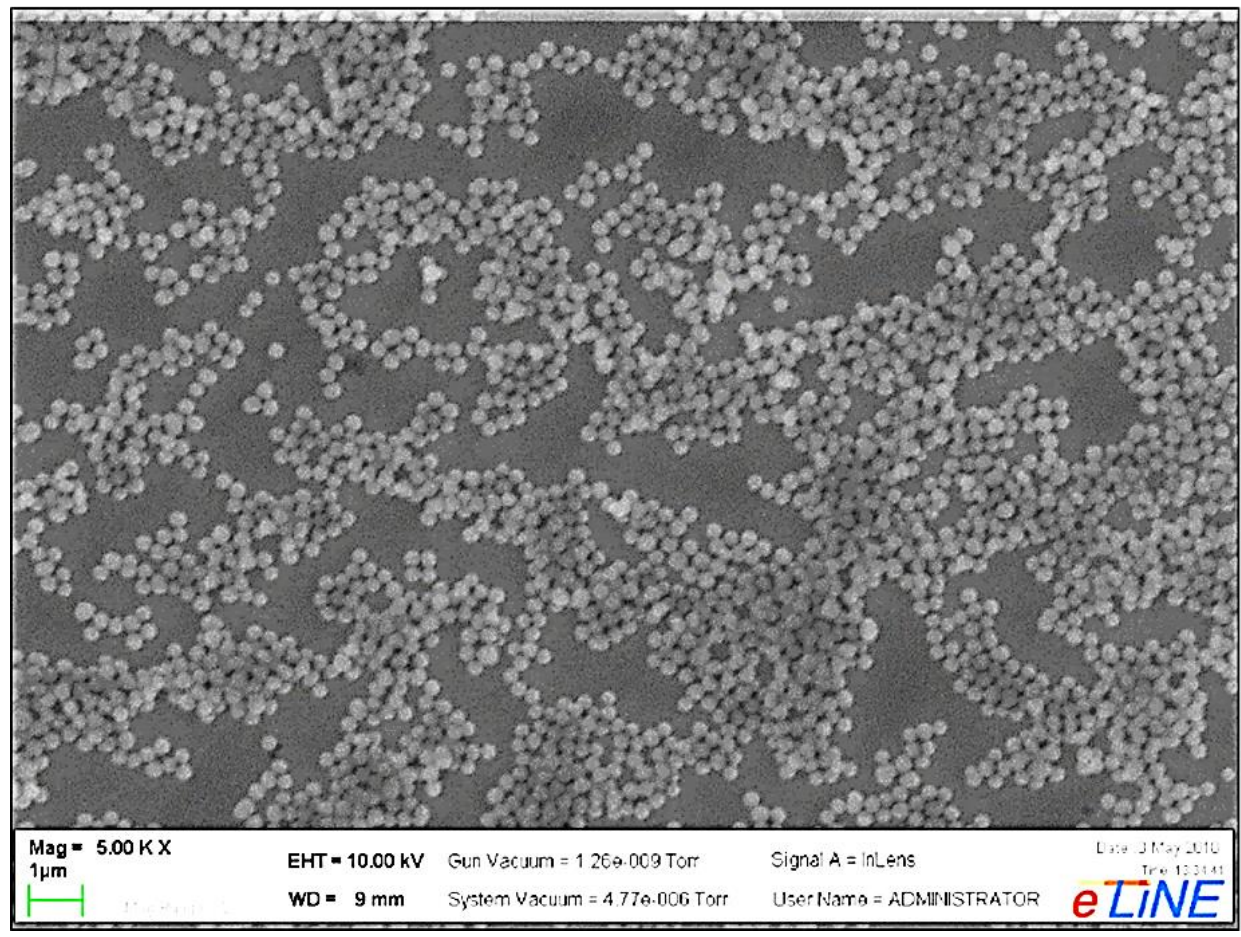

Figure 90. SEM image of $\mathrm{SiO}_{2}$ surface after polystyrene nanosphere spin coating. 
Smooth $\mathrm{SiO}_{2}$ Layer:

Peak-to-Peak Roughness $\approx 10 \mathrm{~nm}$

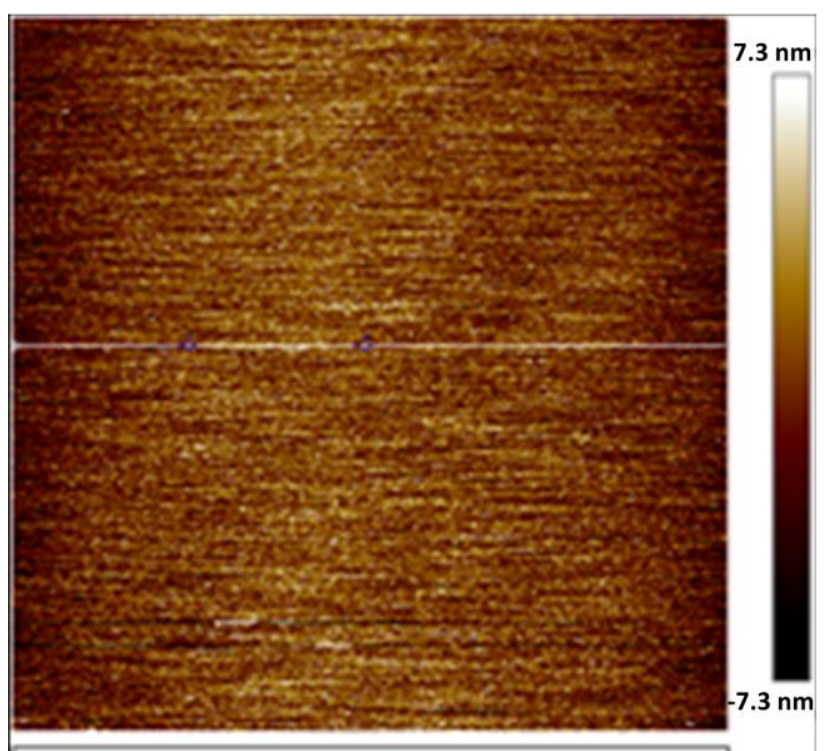

$0 \mu \mathrm{m}$
Baseline $\mathrm{SiO}_{2}$ Layer:

Peak-to-Peak Roughness $\approx 500 \mathrm{~nm}$

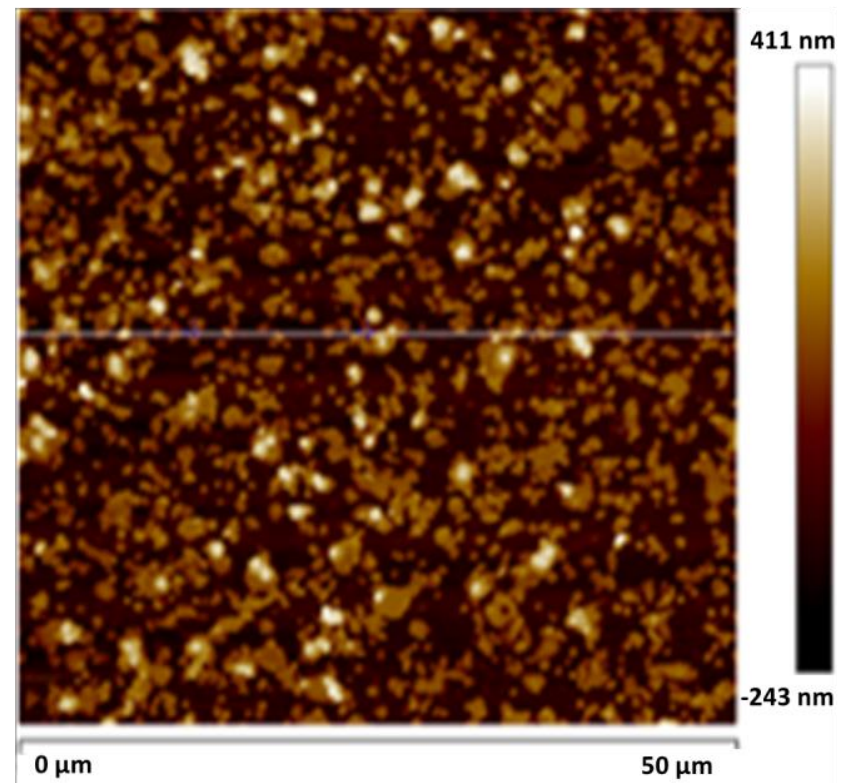

Nanosphere Lithography Roughened $\mathrm{SiO}_{2}$ Layer:

Peak-to-Peak Roughness $\approx 100 \mathrm{~nm}$

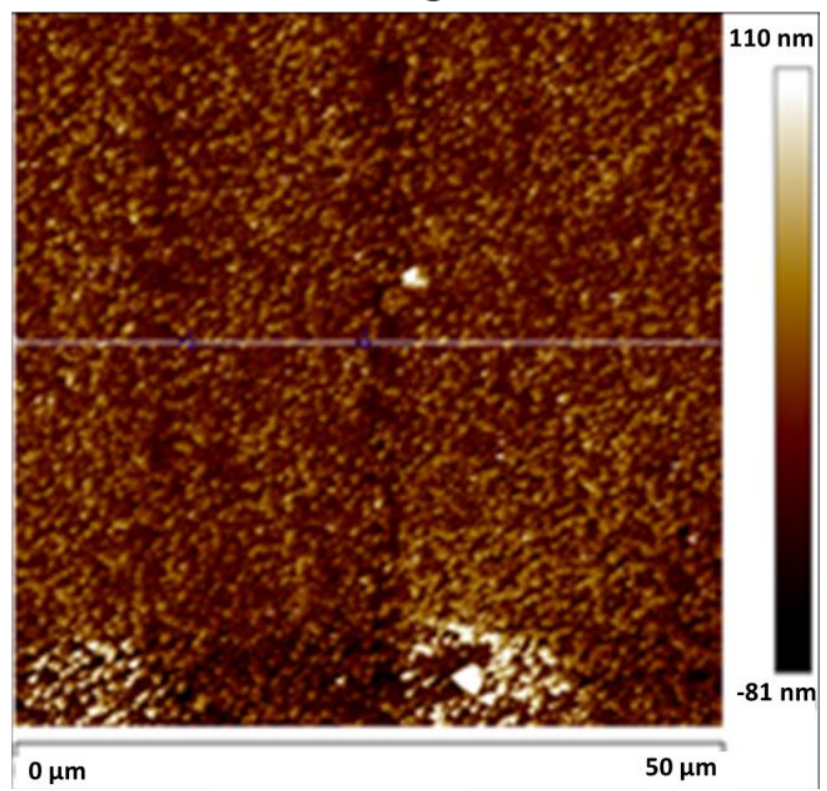

Figure 91. Measured surface roughness maps obtained using AFM of smooth (top-left), baseline roughened (top-right), and nanosphere lithography roughened (bottom) $\mathrm{SiO}_{2}$ on Si substrates. 
Smooth $\mathrm{SiO}_{2}$ Layer: Peak-to-Peak Roughness $\approx 10 \mathrm{~nm}$

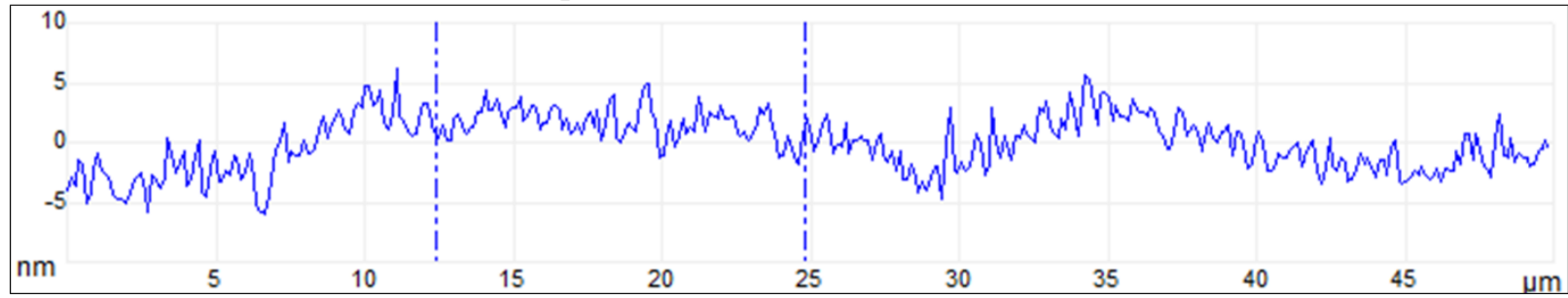

Baseline $\mathrm{SiO}_{2}$ Layer: Peak-to-Peak Roughness $\approx 500 \mathrm{~nm}$

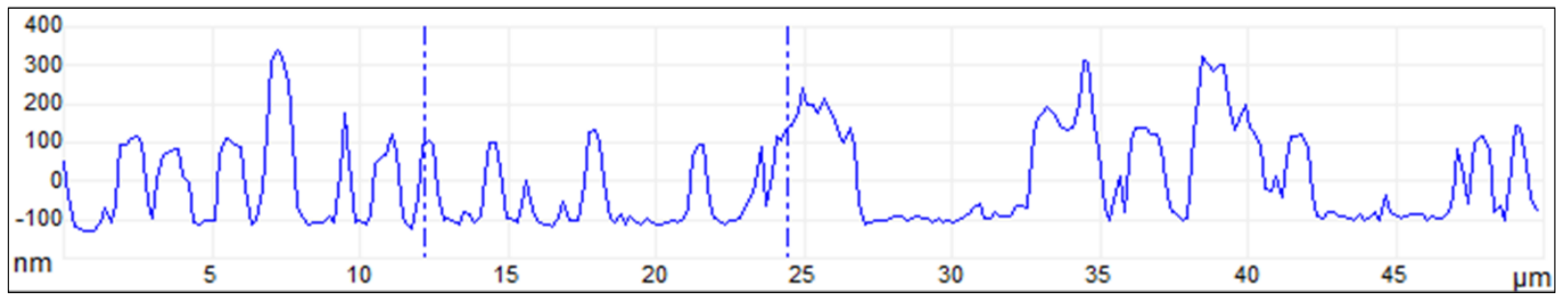

Nanosphere Lithography Roughened $\mathrm{SiO}_{2}$ Layer: Peak-to-Peak Roughness $\approx 100 \mathrm{~nm}$

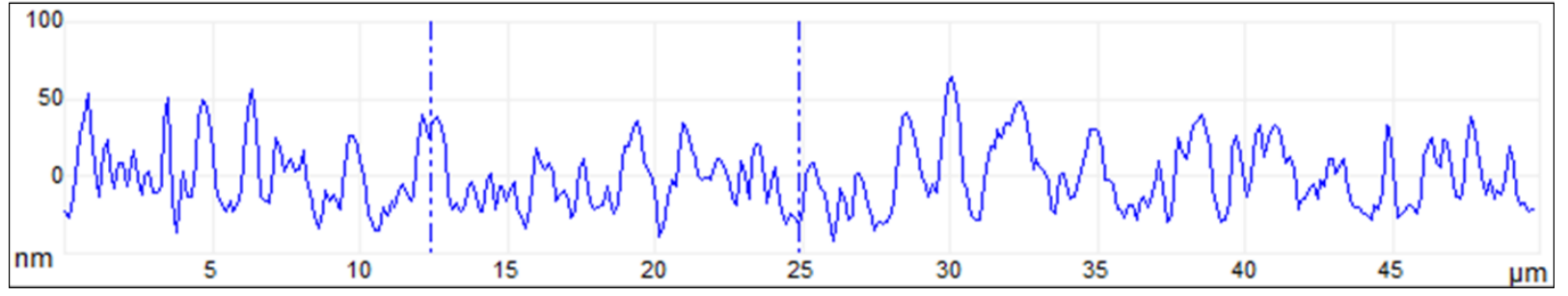

Figure 92. Measured surface profiles obtained using AFM of smooth (top), baseline roughened (middle), and nanosphere lithography roughened (bottom) $\mathrm{SiO}_{2}$ on Si substrates. 


\section{Appendix A References}

[1] Pure Wafer, a WRS Materials Company, “Test Wafers,” 2017. [Online]. Available: https://www.purewafer.com/waferproducts/test. [Accessed Nov. 5, 2018].

[2] M. J. Madou, Fundamentals of Microfabrication: The Science of Miniaturization, Second Edition. CRC Press, 2002.

[3] S. A. Campbell, Fabrication Engineering at the Micro- and Nanoscale, Fourth Edition. Oxford University Press, New York, 2013.

[4] Microchemicals GMBH, "Photoresists," [Online]. Available: https://www.microchemicals.com/products/photoresists.html. [Accessed Nov. 5, 2018].

[5] Suss MicroTec, “MA/BA6 Mask and Bond Aligner,” 2018. [Online]. Available: https://www.suss.com/en/products-solutions/mask-aligner/ma-ba-6. [Accessed Nov. 5, 2018].

[6] Microchemicals GMBH, “AZ® 5214 E, [Online]. Available: https://www.microchemicals.com/products/photoresists/az_5214_e.html. [Accessed Nov. 5, 2018].

[7] Merick, “AZ 5214 E Photoresist,” Merick Performance Materials GmbH. [Online]. Available: https://www.microchemicals.com/micro/az_5214e.pdf. [Accessed Nov. 5, 2018].

[8] H. Fadakar, et al. "Effect of surface roughness on propagation of surface plasmon polaritons along thin lossy metal films," 19th Iranian Conference on Electrical Engineering, 2011. 
[9] Y. Li. Plasmonic Optics Theory and Applications, Tutorial Texts in Optical Engineering, Vol. TT110. SPIE Press. Billingham, WA., 2017.

[10] C. L. Cheung, et al. "Fabrication of nanopillars by nanosphere lithography," Barry Chin Li Cheung Publications. 4, 2006.

[11] W. Wu, D. Dey, and O. G. Memis, "Fabrication of Large Area Periodic Nanostructures Using Nanosphere Photolithography,” Nanoscale Res. Lett. 3, 2008.

[12] O. N. Oliveira Jr, "Langumuir-Blodgett Films-Properties and Possible Application.” Brazilian Journal of Physics, vol. 22, no. 2, 1992.

[13] L. Chitu, et al. "Modified Langmuir-Blodgett deposition of nanoparticlesmeasurement of 2d to 3D ordered arrays," Measurement Science Review, Vol. 10, No. 5, 2010.

[14] ThermoFisher Scientific, "CML Latex Beads, 4\%, $0.3 \mu \mathrm{m}$," ThermoFisher, Inc. [Online]. Available: https://www.thermofisher.com/order/catalog/product/C37237?SID=srch-srpC37237. [Accessed Nov. 5, 2018].

[15] R. R. L. De Oliveira, "Measurement of the Nanoscale Roughness by Atomic Force Microscopy: Basic Principles and Applications,” 2012. [Online]. Available: http://cdn.intechopen.com/pdfs/33450/InTechMeasurement_of_the_nanoscale_roughness_by_atomic_force_microscopy_basic_ principles_and_applications.pdf. [Accessed Nov. 5, 2018]. 


\section{Appendix B: Surface Plasmon Resonance Numerical Models}

\section{Quasi-static Calculation of the Scattering, Absorption, and Extinction Cross-sections and Q-Factors for Surface Plasmon Resonant Discs}

I wrote the following MATLAB script to calculate the surface plasmon resonant Q-factors and cross-sections for oblate spheroids, which was used as a first approximation for disc calculations [1-3].

\%Surface Plasmon Resonance of an Oblate Spheroid (Disc) \%Author: Justin Grayer

\%Date: $11 / 05 / 2018$

\%This script was used to calculate the scattering, absorption, and \%extinction cross-sections and Q-factors for Surface plasmon resonanct \%discs using the quasi-static method

clear variables; close all;clc;

$\%$ Assume a plane wave is polarized along the $\mathrm{x}$-direction

$\mathrm{a}=(0.5 / 2) ; \%$ The disc radius in microns

$\mathrm{b}=(0.5 / 2) ; \%$ The disc radius in microns

$c=0.02 / 2 ; \%$ The disc thickness in microns

$\%$ Define the effective index of refractoin for the disc host material n_eff $=5.07$; \% Index of Refraction of PbSe

rasp $=\mathrm{a} / \mathrm{c} ; \%$ Calculate the disc aspect ratio ecc $=\operatorname{sqrt}\left(1-1 /\right.$ rasp $\left.^{\wedge} 2\right) ; \%$ Calculate the disc eccentricity g_ecc $=\operatorname{sqrt}\left(1-e_{-1}^{\wedge} 2\right) / e c c ; \%$ Simplificaiton constant for calculating

$\%$ Calculate shape factor in $\mathrm{x}$-direction

$\mathrm{Sx}=\left(\mathrm{g} \_\right.$ecc $\left./\left(2 * \mathrm{ecc}^{\wedge} 2\right)\right) *\left((\mathrm{pi} / 2)-\operatorname{atan}\left(\mathrm{g} \_\right.\right.$ecc $\left.)\right)-\left(\mathrm{g} \_\right.$ecc $\left.{ }^{\wedge} 2 / 2\right)$;

$\%$ Calculate shape factor in $y$-direction $\mathrm{Sy}=\mathrm{Sx}$;

$\%$ Calculate shape factor in z-direction $\mathrm{Sz}=(1-\mathrm{Sx}) / 2$;

\%Loop through all wavelengths of interest (From 3 - 5.5 microns as shown)

for ii $=1: 1000 \%$ Number of iteration steps lambda (ii) $=2.99749+0.00251 * \mathrm{ii} ; \%$ Wavelength in microns

$\%$ Complex index of refraction loaded from the metal functions entitiled 
$\% "$ Drude(metal)" for the current wavelength. Au shown.

m_Au(ii) $=$ DrudeAu(lambda(ii)); \% Index of refraction of the metal

eps_m_Au(ii) $=$ m_Au(ii)^2; \%Complex permittivity of the metal

$\%$ Calculate the polarizability in the $\mathrm{x}-, \mathrm{y}-$, and $\mathrm{z}$-directions

alpha_x(ii) $=\left(4 *\right.$ pi $^{*} \mathrm{a}^{*} \mathrm{~b}^{*} \mathrm{c} *($ eps_m_Au(ii)-n_eff^2) $) / \ldots$

$\left(3 *\left(n \_\right.\right.$eff^ $2+S x^{*}($ eps_m_Au(ii)-n_eff^2)) $)$;

alpha_y(ii) $=\left(4 *{ }^{*}{ }^{*}{ }^{*}{ }^{*} b^{*} c *\left(e p s \_m \_A u(i i)-n \_e f f \wedge 2\right)\right) / .$.

$\left(3^{*}\left(\mathrm{n} \_\right.\right.$eff^ $2+\mathrm{Sy} *($ eps_m_Au(ii)-n_eff^2)) ;

alpha_z(ii) $=\left(4 *\right.$ pi $^{*} \mathrm{a}^{*} \mathrm{~b}^{*} \mathrm{c}^{*}($ eps_m_Au(ii)-n_eff^2))/...

$\left(3 *\left(n \_e f f^{\wedge} 2+S z^{*}(\right.\right.$ eps_m_Au(ii)-n_eff^2) $\left.)\right)$;

$\%$ Define the wavenumber

$\mathrm{k}(\mathrm{ii})=\mathrm{n} \_$eff^ $2 * 2 * \mathrm{pi} /(\operatorname{lambda}(\mathrm{ii}))$;

$\%$ Calculate the scatterinng, absorption, and extinction Q factors in

$\%$ the $\mathrm{x}$-direction. Can be expanded to $\mathrm{y}$ - and $\mathrm{z}$ - directions by

$\%$ replacing the complex polarizability.

Qscat_x $(\mathrm{ii})=\left(\mathrm{k}(\mathrm{ii})^{\wedge} 4 * \operatorname{abs}(\text { alpha_x }(\mathrm{ii}))^{\wedge} 2 /(6 * \mathrm{pi} * \mathrm{a} * \mathrm{~b})\right)$;

Qabs_x $($ ii $)=(k(i i) * i m a g($ alpha_x(ii))/(pi*a*b) $)$;

Qext_x(ii) $=$ Qscat_x(ii)+Qabs_x(ii);

$\%$ Calculate the scattering, absorption, and extinction cross-sections.

$\%$ Can be expanded to $\mathrm{y}$ - and $\mathrm{z}$ - directioins by replace the

$\%$ polarizability.

Cscat_x $($ ii $)=\left(\mathrm{k}(\mathrm{ii})^{\wedge} 4 * \operatorname{abs}(\text { alpha_x(ii) })^{\wedge} 2 /(6)\right)$;

Cabs_x $($ ii $)=(\mathrm{k}(\mathrm{ii}) * \mathrm{imag}(\operatorname{alpha} \mathrm{x}(\mathrm{ii})))$;

Cext_x $($ ii $)=$ Cscat_x $($ ii $)+C a b s \_x($ ii $)$;

end

$\%$ Plot the wavelength dependence of the extinction

$\%$ cross-section in units of $\mathrm{cm}^{\wedge} 2$.

figure

plot(lambda, Cext_x*1e-8, 'LineWidth',2)

title(['Normalized Extinction Cross-Section for a Single Pt Nanodisc(Aspect = ', num2str(rasp,3),') at $\mathrm{PbSe} / \mathrm{SiO} \_2$ Interface'])

xlabel('Wavelength ('mum)')

ylabel('Cross-Section $\left.\left(\mathrm{cm}^{\wedge} 2\right)^{\prime}\right)$

set(gca,'FontSize',16)

\section{Calculation of the Dispersion Relations of Drude Metals}

I wrote the following MATLAB script to calculate the dispersion relations of the metals examined in this investigation. These functions were derived from Rakic's treatment [4]. 


\section{$\underline{\text { Silver (Ag) }}$}

$\%$ Calculate the dispersion relations of silver

$\%$ Justin Grayer

$\% 11 / 05 / 2018$

$\%$ Takes in wavelength ("lambda") in eV and returns complex index of \%refraction.

function $[\mathrm{n}]=$ Drude Ag(lambda)

$\%$ Oscillator Strength [eV]

$\mathrm{f}=[0.845,0.065,0.124,0.011,0.840,5.646] ; \%[\mathrm{eV}]$

$\%$ Damping constant per oscillator[eV]

gamma $=[0.048,3.886,0.452,0.065,0.916,2.419]$;

$\%$ Oscillator Frequency

$\mathrm{ww}=[0.816,4.481,8.185,9.083,20.29] ; \%[\mathrm{eV}]$

$\%$ Plasma Frequency $[\mathrm{eV}]$

$\mathrm{wp}=9.01$

$\%$ Energy of input wavelength $[\mathrm{eV}]$

$\mathrm{w}=1.24 / \mathrm{lambda}$;

$\%$ Plasm frequency for intraband transitions $[\mathrm{eV}]$

omegap $=\mathrm{f}(1)^{\wedge}(1 / 2)^{*} \mathrm{wp}$;

$\%$ Calculate metal permittivity for given wavelength

for $\mathrm{ii}=0: 5$

if ii $==0$

eps $=1-$ omegap $^{\wedge} 2 /\left(\mathrm{w} *\left(\mathrm{w}+1 * 1 \mathrm{i}^{*} \operatorname{gamma}(\mathrm{ii}+1)\right)\right)$;

else

eps $=$ eps $+\mathrm{f}(\mathrm{ii}+1)^{*} \mathrm{wp}^{\wedge} 2 /\left(\left(\mathrm{ww}(\mathrm{ii})^{\wedge} 2-\mathrm{w}^{\wedge} 2\right)-1 \mathrm{i}^{*} \mathrm{w}^{*} \operatorname{gamma}(\mathrm{ii}+1)\right)$;

end

end

$\%$ Returns the complex index of refraction of Ag for given wavelength

$\mathrm{n}=\operatorname{sqrt}($ eps $)$;

\section{$\underline{\text { Aluminum (Al) }}$}

$\%$ Calculate the dispersion relations of Aluminum

$\%$ Justin Grayer

$\% 11 / 05 / 2018$

$\%$ Takes in wavelength ("lambda") in eV and returns complex index of

\%refraction.

function $[\mathrm{n}]=$ DrudeAl(lambda)

$\%$ Oscillator Strength [eV]

$\mathrm{f}=[0.523,0.227,0.050,0.166,0.030]$;

$\%$ Damping constant per oscillator[eV]

gamma = [0.047, 0.333, 0.312, 1.351, 3.382];

$\%$ Oscillator Frequency [eV]

$\mathrm{wW}=[0.162,1.544,1.808,3.473]$;

$\%$ Plasma Frequency $[\mathrm{eV}]$ 


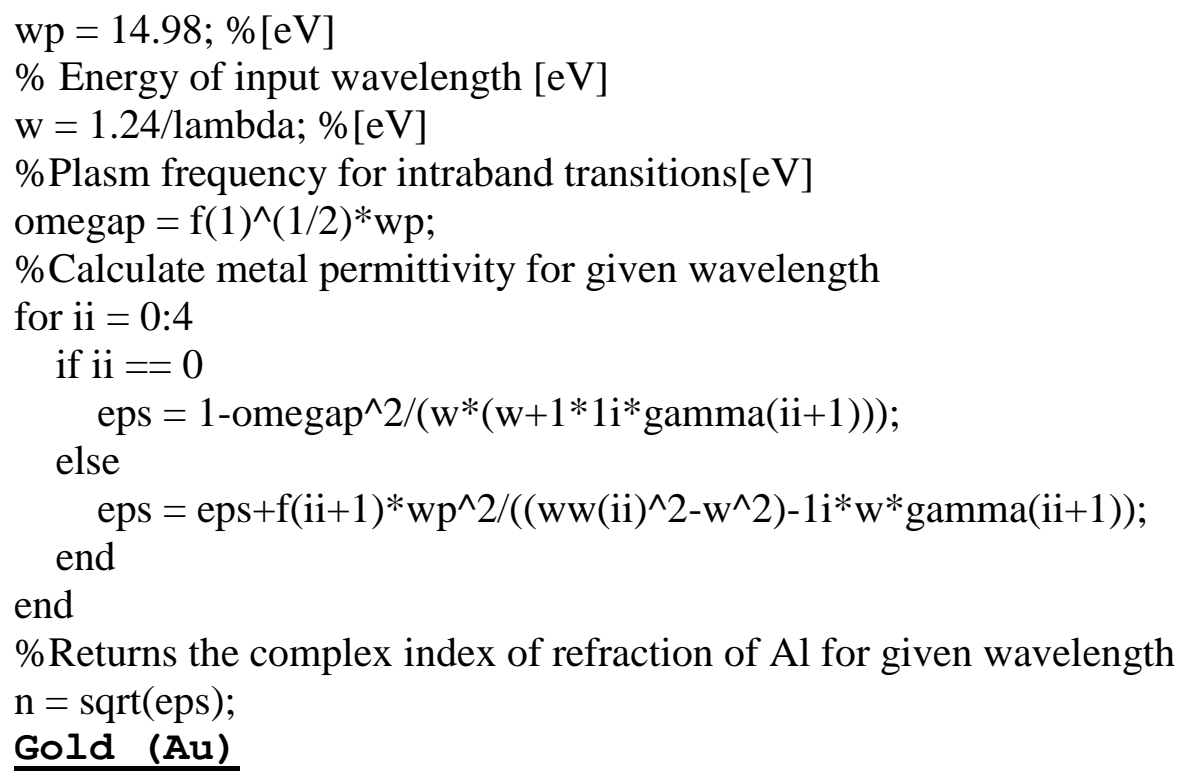

$\%$ Calculate the dispersion relations of Gold

$\%$ Justin Grayer

$\% 11 / 05 / 2018$

\% Takes in wavelength ("lambda") in eV and returns complex index of \%refraction.

function $[\mathrm{n}]=$ DrudeAu(lambda)

$\%$ Oscillator Strength [eV]

$\mathrm{f}=[0.024,0.010,0.071,0.601,4.384]$;

$\%$ Damping constant per oscillator[eV]

gamma $=[0.241,0.345,0.870,2.494,2.214]$;

$\%$ Oscillator Frequency

$\mathrm{wW}=[0.415,0.830,2.969,4.304,13.32]$;

$\%$ Plasma Frequency $[\mathrm{eV}]$

$\mathrm{wp}=9.03$

$\%$ Energy of input wavelength [eV]

$\mathrm{w}=1.24 /$ lambda;

$\%$ Calculate metal permittivity for given wavelength

for $\mathrm{ii}=0: 5$

$$
\text { if } \text { ii }==0
$$

$$
\text { eps }=1-\left(0.760 * w^{\wedge} 2 /\left(w^{\wedge} 2+1 i^{*} 0.053 * w\right)\right)
$$

else

$$
\text { eps }=e p s+f(i i)^{*} w p^{\wedge} 2 /\left(w w(i i)^{\wedge} 2-w^{\wedge} 2-1 i^{*} w^{*} g a m m a(i i)\right) ;
$$
end

end

$\%$ Returns the complex index of refraction of $\mathrm{Au}$ for given wavelength $\mathrm{n}=\operatorname{sqrt}(\mathrm{eps})$; 


\section{Nickel (Ni)}

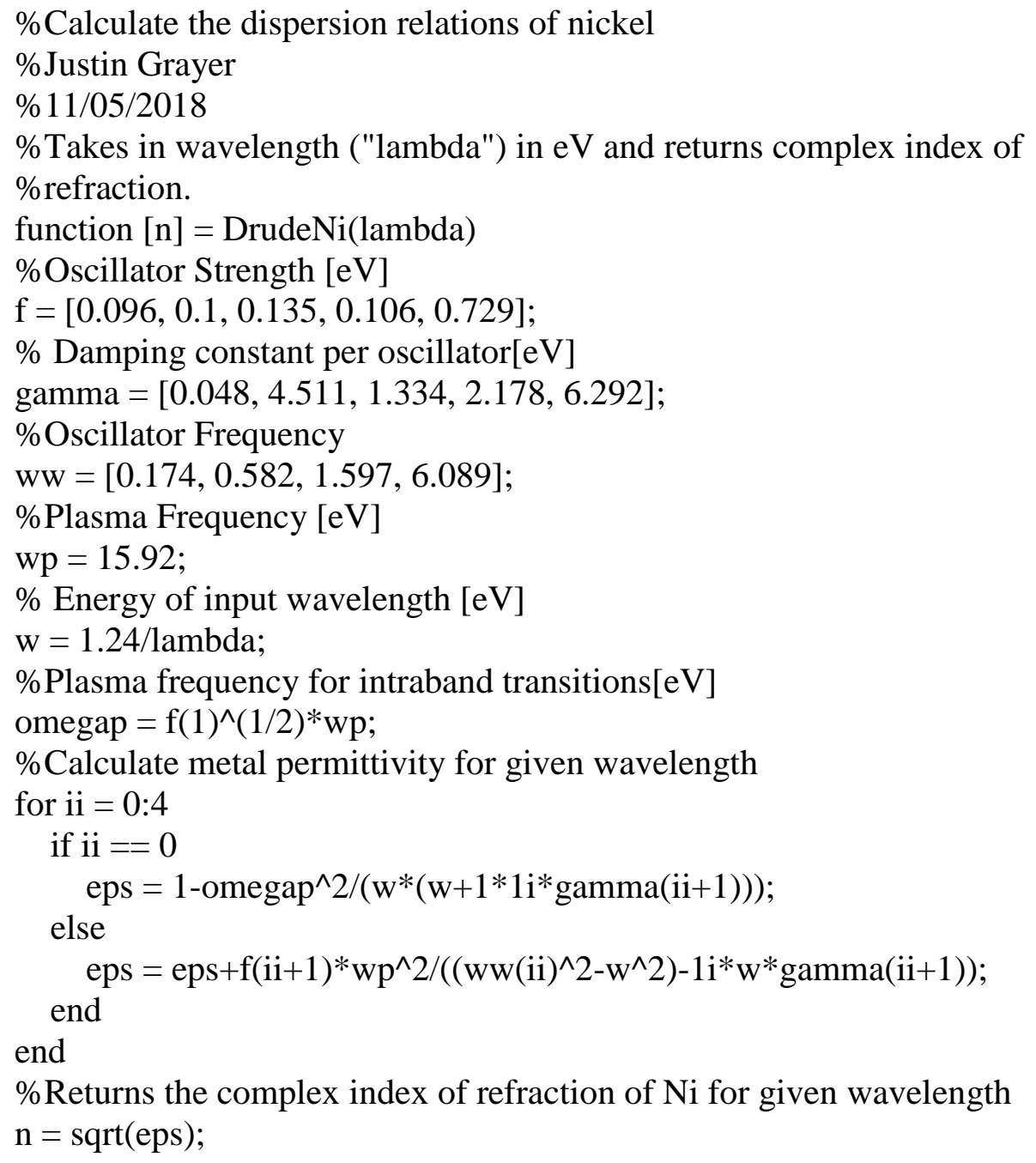

\section{Palladium (Pd)}

$\%$ Calculate the dispersion relations of palladium

$\%$ Justin Grayer

$\% 11 / 05 / 2018$

\% Takes in wavelength ("lambda") in $\mathrm{eV}$ and returns complex index of $\%$ refraction.

function $[\mathrm{n}]=$ DrudePd(lambda)

$\%$ Oscillator Strength [eV]

$\mathrm{f}=[0.330,0.649,0.121,0.638,0.453]$;

$\%$ Damping constant per oscillator[eV]

gamma $=[0.008,2.950,0.555,4.621,3.236]$;

$\%$ Oscillator Frequency [eV]

$\mathrm{wW}=[0.336,0.501,1.659,5.715]$; 


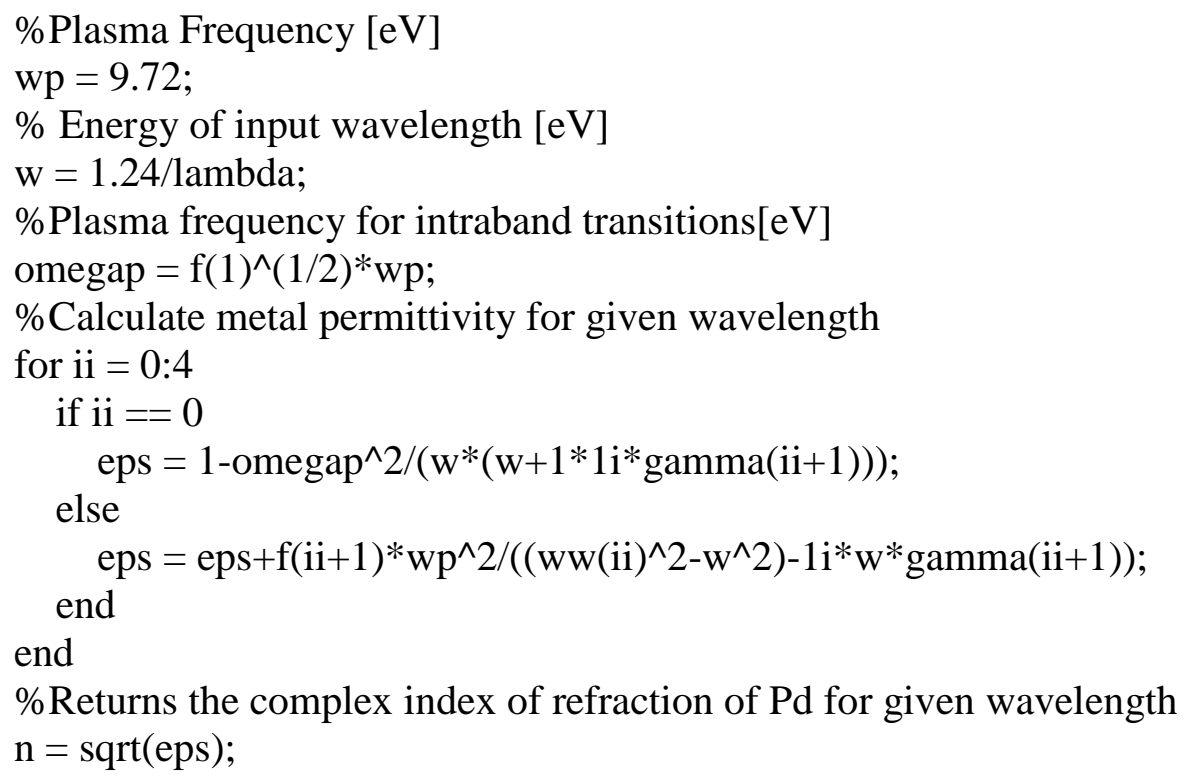

\section{Platinum (Pt)}

$\%$ Calculate the dispersion relations of platinum

$\%$ Justin Grayer

$\% 11 / 05 / 2018$

\% Takes in wavelength ("lambda") in eV and returns complex index of \%refraction.

function $[\mathrm{n}]=$ DrudePt(lambda)

$\%$ Oscillator Strength [eV]

$\mathrm{f}=[0.333,0.191,0.659,0.547,3.576]$;

$\%$ Damping constant per oscillator $[\mathrm{eV}]$

gamma $=[0.080,0.517,1.838,3.668,8.517]$;

$\%$ Oscillator Frequency [eV]

$\mathrm{ww}=[0.780,1.314,3.141,9.249]$;

$\%$ Plasma Frequency [eV]

wp $=9.59$;

$\%$ Energy of input wavelength $[\mathrm{eV}]$

$\mathrm{w}=1.24 . /$ lambda;

$\%$ Plasma frequency for intraband transitions $[\mathrm{eV}]$

omegaP $=\mathrm{f}(1)^{\wedge}(0.5)^{*} \mathrm{wp}$;

$\%$ Calculate metal permittivity for given wavelength

for $\mathrm{ii}=0: 4$

if ii $==0$

$$
\text { eps }=1-\text { omegaP }{ }^{\wedge} 2 /\left(\mathrm{w}^{*}\left(\mathrm{w}+1 \mathrm{i}^{*} \operatorname{gamma}(\mathrm{ii}+1)\right)\right) \text {; }
$$

else

eps $=e p s+f(i i+1)^{*} w^{\wedge} 2 /\left(w w(i i)^{\wedge} 2-w^{\wedge} 2-1 i^{*} w^{*}\right.$ gamma(ii+1)); end

end

\%Returns the complex index of refraction of $\mathrm{Pt}$ for given wavelength 
$\mathrm{n}=\operatorname{sqrt}(\mathrm{eps})$

\section{Surface Plasmon Propagation Distance, Phase Velocity, and Lifetime}

I wrote the following MATLAB script to calculate the surface plasmon propagation distance, phase velocity, and lifetime for idealized metals within $\mathrm{PbSe}$ [1].

$\%$ Surface plasmon parameters for metals

$\%$ Justin Grayer

$\% 11 / 05 / 2018$

$\%$ Calculates the surface plasmon propgation distance, phase velocity, and \%lifetime for a given metal.

clear variables; close all; clc;

$\%$ Speed of light $(\mathrm{m} / \mathrm{s})$

$\mathrm{c}=3 \mathrm{e} 8$

\%Index of effective refractive index of host material(PbSe shown)

n_eff $=5.07$;

$\%$ Calculate prermittivity of host materials

eps_eff $=$ n_eff^2;

\%Loop through all wavelengths of interest (From 2 - 6 microns shown)

for $\mathrm{ii}=1: 40$

$\%$ Define wavelength (microns)

lambda $(\mathrm{ii}, 1)=1.99+0.1 * \mathrm{ii}$;

\%Define frequency $(\mathrm{Hz})$

omega(ii,1) $=2 *$ pi*c/lambda(ii,1);

$\%$ Define wavenumber(microns ${ }^{\wedge}-1$ )

$\mathrm{k} 0(\mathrm{ii}, 1)=2 * \mathrm{pi} / \mathrm{lambda}(\mathrm{ii}, 1)$; 
\%Load in complex index of refraction of the metal (Pt shown)

m_metal_Pt $($ ii, 1$)=\operatorname{DrudePt}(\operatorname{lambda}(\mathrm{ii}, 1))$;

$\%$ Calculate complex permittivity

eps_m_Pt(ii,1) = m_metal_Pt(ii,1)^2;

$\%$ Calculate surface plasmon propgation distance (microns)

dsp_Pt(ii,1) $=2 *$ real (eps_m_Pt(ii,1) $)^{\wedge} 2 /($ eps_eff^(3/2)*imag(eps_m_Pt(ii,1))*k0(ii,1));

$\%$ Calculate surface plasmon phase velocity (microns/s)

Vsp_Pt(ii,1) $=($ c/n_eff $) *(1-($ eps_eff $/(2 *$ real $($ eps_m_Pt(ii, 1$)))))$;

$\%$ Calculate surface plasmon lifetime (s)

tau_sp_Pt(ii,1) =

$2 *$ real(eps_m_Pt(ii,1) $)^{\wedge} 2 /($ omega(ii,1)*eps_eff*imag(eps_m_Pt(ii,1)));

end

$\%$ Plot surface plasmon propgation distance

figure

$\operatorname{subplot}(1,3,1)$

hold on

plot(lambda, dsp_Pt, 'o','MarkerEdge', [ $\left.\begin{array}{lll}0 & 0.5 & 0\end{array}\right]$, 'MarkerFace',[1 11 1], 'LineWidth', 2.5)

title('SP Propagation Distance')

xlabel('Wavelength \mum')

ylabel('Surface Plasmon Propagation Distance (Imum)')

set(gca,'FontSize',16)

hold off

$\%$ Plot surface plasmon phase velocity

$\operatorname{subplot}(1,3,2)$

hold on

plot(lambda, Vsp_Pt/c, 'o', 'MarkerEdge', [0 0.5 0], 'MarkerFace',[l 11 1 1 ,'LineWidth', 2.5) 
title('SP Relative Phase Velocity')

xlabel('Wavelength Imum')

ylabel('Relative Surface Plasmon Phase Velocity (V_s_p/c)')

set(gca,'FontSize',16)

hold off

$\%$ Plot surface plasmon lifetime

$\operatorname{subplot}(1,3,3)$

hold on

plot(lambda, tau_sp_Pt/1e-9, 'o', 'MarkerEdge', [0 0.5 0], 'MarkerFace',[1 1

1],'LineWidth', 2.5)

title('SP Lifetime')

xlabel('Wavelength Imum')

ylabel('Surface Plasmon Lifetime (ns)')

set(gca,'FontSize',16)

hold off 


\section{Appendix B References}

[1] D. Sarid and W. Challner, Modern Introduction to Surface Plasmons: Theory, Mathematical Modeling, and Application, New York, NY: Cambridge University Press, 2010.

[2] A. A. Toropov and T. V. Shubina, Plasmonic Effects in Metal-Semiconductor Nanostructures, Great Clarendon, Oxford, United Kingdom: Oxford University Press, 2015.

[3] A. Battaglia, et al. "Light scattering by Gaussian particles: Rayleigh-ellipsoid approximation,” J. of Quantitative Spectroscopy and Radiative Transfer, 63, 1999.

[4] Rakic', et al. "Optical Properties of Metallic Films for Vertical Cavity Optoelectronic Devices,” Applied Optics, Vol. 37, No. 22, 1998. 


\section{Appendix C: Infrared Sensor System Considerations}

Long range imaging systems have become an important tool for situational awareness and detailed examination of the surrounding environment. Examples of their use include advanced threat warning, surveillance and reconnaissance, and sensing of chemical and biological agents. Electro-optical and infrared systems (EO/IR) cover five major bands of the electromagnetic spectrum. These include visible $(0.4 \mu \mathrm{m}-0.7 \mu \mathrm{m})$, near-infrared $(0.7 \mu \mathrm{m}-1 \mu \mathrm{m})$, short-wave infrared (SWIR; $1 \mu \mathrm{m}-3 \mu \mathrm{m})$, mid-wave infrared (MWIR; $3 \mu \mathrm{m}-6 \mu \mathrm{m}$ ), and long-wave infrared (LWIR; $6 \mu \mathrm{m}-14 \mu \mathrm{m}$ ) [1]. Imaging systems are designed for specific applications, with the primary design choice being the spectral band over which the system will operate. For instance, consider a commercial camera intended for consumer photography. This system would be designed for the visible regime, in which the light from either the sun or an artificial source would be reflected off of a subject into the imaging optics and focused onto the detector. In contrast, an infrared system, specifically those designed for MWIR or LWIR, are designed to collect the emitted electromagnetic radiation from the subject or scene. This emitted radiation is determined by the surface temperature of the subject, and is present in all objects with temperatures above absolute zero. Note that due to its temperature dependent nature, infrared radiation is typically associated with thermal radiation.

The examples above show an important distinction between the visible spectrum, associated with the photopic response of the human eye, and the infrared spectrum. Typically visible systems collect and image the reflected light off of a subject, while infrared systems primarily collect thermally emitted radiation. There is another important difference between the two bands, which is the attenuation due to the atmosphere. When 
considering atmospheric effects, the aforementioned five bands of interest are divided into what are referred to as atmospheric windows [1, 2-3]. Imaging systems are designed within these windows to mitigate the attenuation from $\mathrm{CO}_{2}$, moisture, and other particulates within the atmosphere. Figure 93 shows the atmospheric transmission over a $1 \mathrm{~km}$ horizontal range at an altitude of $5 \mathrm{~km}[4]$.

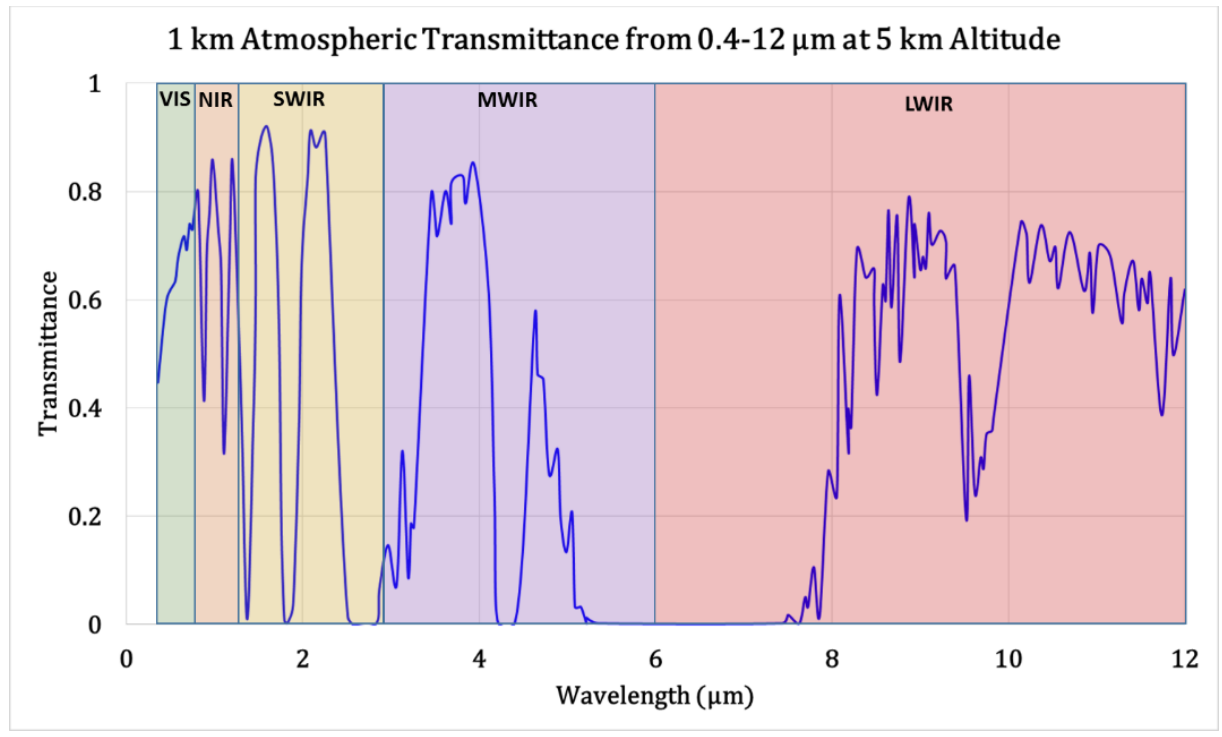

Figure 93. Transmission spectra from visible to LWIR regions of the electromagnetic spectrum over a $1 \mathrm{~km}$ range at an altitude of $5 \mathrm{~km}$ [4]. Each spectral region contains atmospheric windows through which electromagnetic radiation can propagate without substantial absorption from atmospheric particulates.

For example, in figure 93 the transmission in the visible spectrum is relatively high, with greater than $70 \%$ of photons making it through the $1 \mathrm{~km}$ distance. However, in the SWIR, MWIR, and LWIR bands there are several drops in transmission due to atmospheric absorption. For systems primarily concerned with sensing the thermal emission of objects within a scene, the MWIR and LWIR regions are of primary interest. For systems sensitive to these regions, the solar background is relatively low, and can even be absent if the system is intended for night-time operation. Systems employing the 
MWIR and LWIR regimes are typically concerned with what is known as black body radiation.

\section{Black Body Radiation}

A black body is an idealization of a perfect emitter and absorber, in which the spectral band of emission or absorption is related to the temperature of the object. The amount of thermal radiation emitted by a real object can be quantified through Planck's law, which relates the spectral emittance, with units of $W / m^{2} s r \mu m$, of an object to its temperature and emitted wavelength by equation D.1,

$$
M(\lambda, T)=\epsilon(\lambda) \frac{c_{1}}{\lambda^{5}} \frac{1}{\exp \left(\frac{c_{2}}{\lambda T}\right)-1}
$$

where $c_{1}$ is a constant with a value of $3.7418 \times 10^{8} \mathrm{~W}-\mu \mathrm{m}^{4} / \mathrm{m}^{2}, \mathrm{c}_{2}$ is another constant with a value of $1.4388 \times 10^{4} \mu \mathrm{m}-\mathrm{K}, \lambda$ is the wavelength in $\mu \mathrm{m}$, and $\mathrm{T}$ is the temperature in $\mathrm{K}$ [1-2]. The emissivity $\epsilon$ is a measure of how well an object emits radiation, where an $\epsilon$ equal to 1 represents a perfect black body over all wavelengths. Another important relationship that can be derived from Planck's law is Wien's law of displacement in which the peak wavelength of a thermal emitter is related to the temperature of that emitter by equation D.2.

$$
\lambda_{\text {peak }} \cong \frac{2898 \mu \mathrm{m} \cdot K}{T}
$$

As an example, consider the sun which has an average surface temperature of $5778 \mathrm{~K}$. The peak wavelength emitted by the sun is $502 \mathrm{~nm}$, which is in the green portion of the visible spectrum. For infrared applications, a $300 \mathrm{~K}$ blackbody, the spectrum of which is shown in figure 94, is often considered because it approximates the background emission 
at room temperature. With a peak wavelength of $9.8 \mu \mathrm{m}$, the background emission is significant at $300 \mathrm{~K}$. Even in the MWIR regime, where the $300 \mathrm{~K}$ emission falls off, the background can overwhelm or degrade the signal of interest. It is this background radiation that limits the performance of infrared detectors, and necessitates the need for a cooling element within the sensing system.

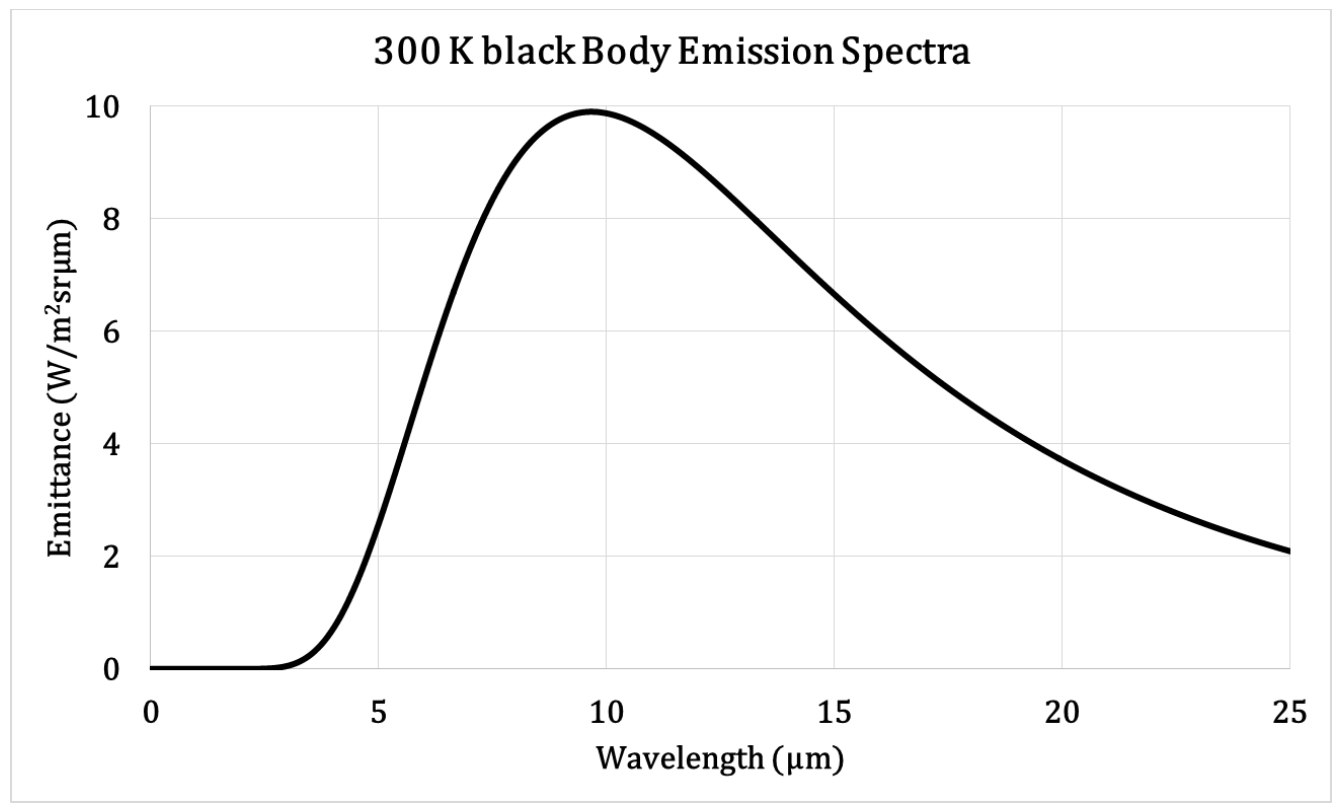

Figure 94. The 300 K black body emission spectrum calculated using Plank's law. Thought the peak of the spectrum is located at $9.8 \mu \mathrm{m}$, there is appreciable emission in the MWIR regime which typically drives the need for a cooling element in infrared sensing systems.

\section{Modern Infrared Imaging Systems}

An infrared imaging system consists of a receiver, detector, and signal processing electronics [1-3]. The optical receiver includes elements such as a turret for pointing at the subject or scene of interest, telescope optics to transfer and focus the photons from the scene to the detector, and various optomechanical assemblies. The optical detector includes the light sensitive material, detector cooling assembly, and read-out integrated circuit (ROIC). The signal processing electronics are closely tied with the ROIC, and can 
include analogue-to-digital conversion, image and digital signal processing, and data interpretation. A block diagram representation of the photon flow from the scene through the imaging system is shown in figure 95. Photons are emitted from the scene, and transmitted to the receiver where some may be absorbed by compounds in the atmosphere. The photons are then further attenuated as they transmit through and are focused by the telescope, which usually consists of both reflective and refractive optics. Photons are imaged onto the detector, which in modern imaging systems is a focal plane array consisting of thousands or even millions of detector elements called pixels.

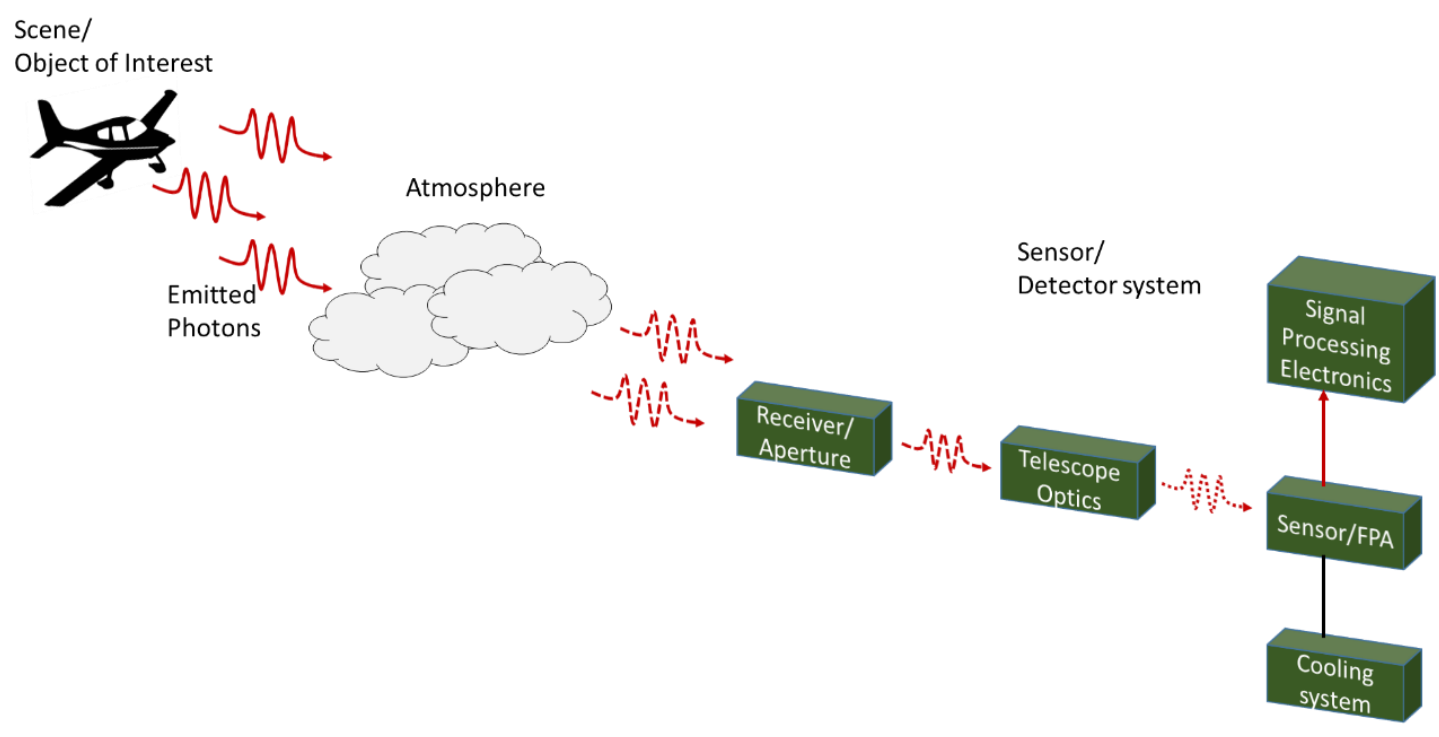

Figure 95. Block diagram showing emitted photons from an object of interest as they transmit through the atmosphere into a long-range sensing system. Photons are attenuated by atmospheric particulates, as well as the optical elements of the sensing system.

The sensitivity of a detector element starts with the material of which it is made.

For instance, in the visible spectrum materials like mercury cadmium telluride ( $\mathrm{HgCdTe})$, cadmium sulfide (CdS), and Si are utilized as detector materials [5]. In the SWIR region $\mathrm{HgCdTe}$, indium gallium arsenide (InGaAs), and lead sulfide ( $\mathrm{PbS})$ are utilized $[2,7]$. For the MWIR band $\mathrm{HgCdTe}$, indium antimonide (InSb), and lead selenide ( $\mathrm{PbSe})$ are 
popular materials $[5,7]$, and in the LWIR region $\mathrm{HgCdTe}$, quantum well infrared photodetectors (QWIP), and vanadium oxide (VOx) are used [8]. All of the aforementioned materials, regardless of the spectral band, are semiconductors, which are governed by the composition and quantum mechanical properties of the material $[3,9$, 10-12]. 


\section{Appendix C References}

[1] J. C. Leachtenauer, and R. G. Driggers, Surveillance and reconnaissance imaging systems: Modeling and Performance Prediction. Norwood, MA: Artech House, Inc, 2004.

[2] K. Kasunic, Optical Systems Engineering. McGraw-Hill Education, 2011.

[3] C. J. Willers, Electro-Optical Systems Analysis and Design, Bellingham, WA: SPIE Press, 2013.

[4] Spectral Sciences, Inc. [online] Available: http://modtran.spectral.com/modtran_home\#plot. [Accessed Sept. 11, 2018]

[5] P. R. Norton, "Photodetectors," in McGraw-Hill's Handbook of Optics, 3rd ed., Vol. 2, New York: McGraw-Hill, 2010, pp. 24.3-24.100.

[6] E. Hergert, "Detectors: Guideposts on the Road to Selection," in the Photonics Directory, 5nd Ed. Laurin Publishing, 2006.

[7] R. A. Smith, "Detectors for Ultraviolet, Visible, and Infrared Radiation," Applied Optics, Vol. 4, No. 6, 1965

[8] A. Rogalski, M. Kopytko, and P. Martyniuk, Antimonide-based Infrared Detectors: A New Perspective, Bellingham, WA: SPIE Press, 2018.

[9] D. A. Neamen, Semiconductor Physics and Devices: Basic Principles, $3^{\text {rd }}$ Ed, New York, NY: McGraw Hill, 2003.

[10] R. A. Serway, C. J. Moses, and C. A. Moyer, "The Solid State," in Modern Physics, $3^{\text {rd }}$ ed, Belmont, CA: Borrks/Cole-Thomson Learning, 2005.

[11] C. Kittel, Introduction to Solid State Physics, Hoboken, NJ: John Wiley and Sons, Inc. 2005. 
[12] M. P. Marder. Condensed Matter Physics, $2^{\text {nd }}$ ed. Hoboken, NJ: John Wiley and Sons, Inc. 2010. 


\section{VITA}

Justin S. Grayer was born to Carla and Carl Grayer in Columbia, Missouri. Upon completing secondary education at Rock Bridge High School, he attended Indian Hills Community College in Ottumwa, Iowa. There, he studied Laser Electro-Optics

Technology, earning an associate of applied science (A.A.S) degree. Upon completion of his degree, Justin was recruited by Raytheon Space and Airborne Systems in El Segundo, CA as a laser technician. After working his way up to Senior Laser Technician, he began his studies again by taking courses on physics as a part-time student at Santa Monica College. He would eventually move back to Columbia, Missouri to pursue an undergraduate degree in physics from the University of Missouri (MU).

Shortly after the start of his first semester at MU, Justin joined Dr. Gavin King's single-molecule biophysics group. There, he was an integral team member in the setup, alignment, and test of the second generation ultra-stable atomic force microscope. Justin graduated with honors, earning a B.S. degree in Physics in 2012. He remained in Dr. King's group, and earned a M.S. Physics in 2013. Following this achievement, Justin transitioned to Dr. Gregory Triplett's group in the MU department of Electrical and Computer Engineering. There, he would study and grow quantum cascade lasers by molecular beam epitaxy.

Justin earned his second Master's degree, this time in engineering, in the summer of 2014. He started a position as a Senior Systems Engineer in the EO/IR Design Department at Raytheon Space and Airborne Systems in El Segundo, CA shortly after graduating. While at Raytheon, he worked on projects such as star trackers, high energy 
laser, and precision photonic oscillators. While working on these projects he was coinventor on three invention disclosures related to holographic imaging systems for wave front sensing and correction, which as of this writing are under review by the United States patent office.

Also while at Raytheon, Justin pursued a professional certificate in optical engineering from University of California-Irvine. He continued to do so when he took a position as a Senior Systems Engineer at Northrop Grumman in Rolling Meadows, IL early in 2016, and would eventually earn the certificate in optical engineering at the beginning of 2017. Justin's projects at Northrop Grumman have included the characterization and test of 3D Geiger-mode APD cameras, field testing of laser illuminated 3D linear mode APD cameras, and systems design work for the LITENING program area. In 2017 he transitioned to the Advanced Concepts group and the role of Physicist. Since then, he has primarily been focused on infrared detectors and infrared countermeasure systems.

While working at Northrop Grumman, Justin re-entered the $\mathrm{PhD}$ program in the Electrical and Computer Engineering Department at the University of Missouri under Dr. Randy Curry. His PhD research was focused on the design and incorporation of optically resonant structures into high operating temperature $\mathrm{PbSe}$ photoconductors for enhanced mid-infrared sensitivity. These structures included surface plasmon resonant structures, interference cavities, and anti-reflection coatings. His work was supported by the Defense Advanced Research Projects Agency (DARPA) and the Air Force Research Laboratory (AFRL) as part of the Wafer-scale Infrared Detectors (WIRED) program. Moreover, in addition to his WIRED teammates, Justin has collaborated with Dr. Mool Gupta's and 
Avik Ghosh's group at the University of Virginia, as well as Philippe Guyot-Sionnest's group at the University of Chicago. As of this writing, Justin continues to work as a Physicist at Northrop Grumman, where his focus and passion are on electro-optical systems for infrared countermeasures. Justin is also an adjunct professor in the Lasers, Photonics, and Optics department at the College of Lake County in Grayslake, IL, where he teaches aspiring students the skills they need to either become laser technicians or pursue higher educational goals. 